\title{
ADVANCED DIAGNOSTIC TECHNIQUES FOR THREE-PHASE SLURRY BUBBLE COLUMN REACTORS (SBCR)
}

\author{
Final Technical Report \\ DE-FG-26-99FT40594
}

\section{Principal Investigator (PI):}

M.H. Al-Dahhan

Associate Professor and Associate Director

Chemical Reaction Engineering Laboratory

\author{
Washington University \\ Department of Chemical Engineering \\ Campus Box 1198 \\ One Brookings Drive \\ St. Louis, Missouri 63130 \\ Fax: 314-935-4832 \\ Phone: 314-935-7187 \\ E-mail: muthanna@che.wustl.edu
}

Ohio State University

Department of Chemical Engineering

140 West $19^{\text {th }}$ Avenue-Room 125

Columbus, Ohio 43210-1180

Fax: 614-292-3769

Phone: 614-292-7907

E-mail: FAN@er6s1.eng.ohio-state.edu

\author{
M.P. Dudukovic \\ The Laura and William Jens \\ Professor and Chairman \\ Director, Chemical Reaction Engineering \\ Laboratory
}

Washington University

Department of Chemical Engineering

Campus Box 1198

One Brookings Drive

St. Louis, Missouri 63130

Fax: 314-935-4832

Phone: 314-935-6021

E-mail: dudu@poly1.che.wustl.edu

Air Products and Chemicals, Inc.

B. Toseland

\section{Co-Investigators}

Washington University:

Ashfaq Shaikh, N. Rados, David Newton

Ohio State University:

August, 2003

Prepared for the United States Department of Energy

Award No. DE-FG-26-99FT40594

Award Period: July 1, 1999 - June 30, 2003 


\section{$\underline{\text { Disclaimer }}$}

This report was prepared as an account of work sponsored by an agency of the United States Government. Neither the United States Government nor any agency therefore, nor any of their employees, makes any warranty, express or implied, or assumes any legal liability or responsibility for the accuracy, completeness, or usefulness of any information, apparatus, product, or process disclosed, or represents that its use would not infringe privately owned rights. Reference herein to any specific commercial product, process, or service by trade name, trademark, manufacturer, or otherwise does not necessarily constitute or imply its endorsement, recommendation or favoring by the United States Government or any agency thereof. The views and opinions of authors expressed herein do not necessarily state or reflect those of the United States Government or any agency thereof. 


\title{
ADVANCED DIAGNOSTIC TECHNIQUES FOR THREE-PHASE SLURRY BUBBLE COLUMN REACTORS (SBCR)
}

\author{
Final Technical Report \\ DE-FG-26-99FT40594
}

\begin{abstract}
The objectives set for this cooperative project between Washington University (WU), Ohio State University (OSU), and Air Products and Chemicals, Inc. (APCI) to advance the understanding of the Fischer-Tropsch (FT) slurry bubble column reactor hydrodynamics for proper design and scale-up via advanced diagnostic techniques have been accomplished successfully despite the unexpected challenging technical difficulties in implementing the advanced techniques in high pressure stainless steel slurry bubble column.

In this work, a detailed review of the aspects of high pressure phenomena of bubbles in liquids and liquid-solids suspension was performed. All the challenging technical problems mentioned above were resolved and the advanced measurement techniques were successfully used in this project. The effects of reactor pressure, superficial gas velocity, solids loading, and liquid physical properties on the overall gas holdup, holdups distribution, recirculation velocity, turbulent parameters, bubble dynamics (size and rise velocity) were investigated via advanced measurement techniques that includes optical probe, Laser Doppler Anemometry (LDA), Computed Tomography (CT), Computer Automated Radioactive Particle Tracking (CARPT). The findings are discussed and analyzed in this report. In attempt to advance the design and scale-up of bubble columns, new correlations have been developed based on a large bank of data collected at a wide range of operating and design conditions. These correlations are for prediction of radial gas holdup profile, axial liquid velocity profile, overall gas holdup based on Neural Network and gas-liquid mass transfer coefficient.

Despite the noticeable advances made on FT SBCR as a part of this project, there are still many parameters and challenging issues that need to be further and properly investigated and understood before this technology will be readily used for alternative fuel development technology.
\end{abstract}




\title{
ADVANCED DIAGNOSTIC TECHNIQUES FOR THREE-PHASE SLURRY BUBBLE COLUMN REACTORS (SBCR)
}

\author{
Final Technical Report \\ DE-FG-26-99FT40594
}

\section{TABLE OF CONTENTS}

Disclaimer

Abstract

Table Contents

Page No.

List of Figures

Executive Summary

ii

iii

iv

vii

$\mathrm{X}$

1. OVERALL OBJECTIVES 1

1.1 Accomplishments During the First Year 2

1.2 Accomplishments During the Second Year 2

1.3 Accomplishments During the Third Year 3

1.4 Accomplishments During no-cost extension period 3

2. TECHNICAL REVIEW 4

2.1 Variables Affecting Slurry Bubble Column (SBCR)

Performance

2.2 Some aspects of bubble dynamics and hydrodynamic properties

2.3 Models used for FT reactor performance prediction 9

2.4 Physical Properties of FT Systems 12

3. EXPERIMENTAL FACILITY 18

3.1 High pressure and high temperature 2" diameter slurry bubble column

3.2 High pressure 6" diameter slurry bubble column 18

3.3 Dynamic Gas Disengagement (DGD) 19

$\begin{array}{ll}3.4 \text { Fiber Optic Probe } & 19\end{array}$

3.5 Laser Doppler Anemometer (LDA) 20

3.6 Computer Automated Radioactive Particle Tracking (CARPT)

3.7 Computed Tomography (CT) 22

4. RESULTS AND DISCUSSIONS 36

4.1 Hydrodynamics measurements in 2 inch column using DGD, $\Delta \mathrm{P}$ Fluctuation, and Fiber Optic Probe $\quad 36$

4.1.1 Overall gas holdup using DGD 36

4.1.2 Prediction of regime transition using $\Delta \mathrm{P}$ fluctuations 36 
4.1.3 Bubble Size and Bubble Rise Velocity

4.1.4 Averaged liquid velocity and Reynolds stresses measured by LDA using Norpar 15-nitrogen system

4.2 Hydrodynamics Measurements in 6 inch column using CARPT and CT

4.2.1 Results of CT (gas holdup profile)/CARPT (solids axial velocity profile and turbulent parameters) using Air-WaterGlass Beads System

4.2.2 Results of CT (gas holdup profile)/CARPT (liquid axial velocity profile and turbulent parameters) using Air-Water system

4.2.3 Results of CT (gas and solids holdup profile) Using Air-Therminol LT-Glass Beads System

5. SOME ASPECTS OF HIGH-PRESSURE PHENOMENA OF BUBBLES IN LIQUIDS AND LIQUID-SOLID SUSPENSIONS

6. DEVELOPMENT OF A CORRELATION FOR THE PREDICTION OF RADIAL GAS HOLDUP PROFILES IN BUBBLE COLUMNS

7. DEVELOPMENT OF A CORRELATION FOR THE PREDICTION OF AXIAL LIQUID VELOCITY IN BUBBLE COLUMNS

8. DEVELOPMENT OF AN ARTIFICAL NEURAL NETWORK CORRELATION FOR PREDICTION OF OVERALL GAS HOLDUP IN BUBBLE COLUMN REACTORS

9. PREDICTION OF MASS TRANSFER COEFFICIENT IN BUBBLE COLUMNS OPERATED AT HIGH PRESSURE BASED ON ATMOSPHERIC PRESSURE DATA

10 PHASE DISTRIBUTION IN A HIGH PRESSURE SLURRY BUBBLE COLUMN REACTOR VIA COMPUTED TOMOGRAPHY

11. TECHNICAL DIFFICULTIES AND IMPROVEMENTS IN CARPT

12. REFERENCES 66

$\begin{array}{ll}\text { 12.1 Nomenclature } & 67\end{array}$

$\begin{array}{ll}12.2 \text { References } & 68\end{array}$ 
$\begin{array}{ll}\text { 13. } & 73\end{array}$

14. APPENDIX B 144

$\begin{array}{ll}\text { 15. } & 161\end{array}$

16. APPENDIX D 205

$\begin{array}{ll}\text { 17. APPENDIX E } & 213\end{array}$

18. APPENDIX F 222 


\section{LIST OF FIGURES}

$\underline{\text { Figure No. }}$

$\underline{\text { Caption }}$

$\underline{\text { Page No. }}$

Figure 2.1 Effect of Design Parameters, Operating Variables, Phase

Physical Properties and Kinetics on the Slurry Bubble Column

Yield and Selectivity

Figure 3.1 Schematic diagram for high pressure and high temperature $\begin{array}{ll}\text { slurry bubble column } & 28\end{array}$

Figure 3.2 Gas flow sheet for the high pressure 6 inch diameter bubble column

Figure 3.3 Bubble column reactor of 6" without ports used for CARPT/CT measurements. CT1, CT2, CT3 represents the scan levels used in this investigation

Figure 3.4 Bubble column reactor of 6" with ports used for overall gas holdup and DP measurements. CT1, CT2, CT3 represents the scan levels used in this investigation.

Figure 3.5 Typical variation of dynamic pressure gradient with time $\begin{array}{ll}\text { during the bed disengagement process. } & 32\end{array}$

Figure 3.6 Schematic diagram of the U-shaped optical fiber probe 32

Figure 3.7 Laser Doppler Anemometer Setup 33

Figure 3.8 Configuration of the CARPT experimental setup 34

Figure 3.9 Configuration of the CT experimental setup (Kumar, 1994) 35

Figure 4.1 Effect of pressure on overall gas holdup (Nitrogen - Norpar 15

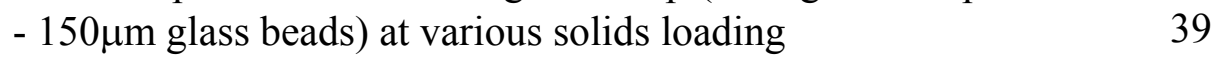

Figure 4.2 Effect of solids loading on gas holdup (Nitrogen - Norpar 15 $150 \mu \mathrm{m}$ glass beads) at various operating pressures

Figure 4.3 Effect of a) solids loading and b) operating pressure on regime transition using Nitrogen - Norpar $15-150 \mu \mathrm{m}$ glass beads in 2" column

Figure 4.4 Effect of solids loading on bubble size using Nitrogen - Norpar $15-150 \mu \mathrm{m}$ glass beads in $2 "$ column at $\mathrm{Ug}=30 \mathrm{~cm} / \mathrm{s}, \mathrm{P}=$ $1.78 \mathrm{MPa}$

Figure 4.5 Effect of solids loading on bubble rise velocity using Nitrogen - Norpar 15 - $150 \mu \mathrm{m}$ glass beads in 2" column at Ug $=30$ $\mathrm{cm} / \mathrm{s}, \mathrm{P}=1.78 \mathrm{MPa}$

Figure 4.6 Averaged liquid velocity measurement at various superficial gas velocities at ambient pressure

Figure 4.7 Tangential Reynolds normal stress at various superficial gas velocities at ambient pressure 
Figure 4.8 Axial Reynolds normal stress at various superficial gas velocities at ambient pressure

Figure 4.9 Effect of superficial gas velocity on gas holdup profile (airwater-glass beads $150(\mu \mathrm{m})$ in $6 "$ column with $9.1 \%$ vol. solids loading at $0.1 \mathrm{MPa}$

Figure 4.10 Effect of superficial gas velocity on solids axial velocity profile (air-water-glass beads $150 \mu \mathrm{m}$ ) in 6" column with 9.1 $\%$ vol. solids loading at $0.1 \mathrm{MPa}$

Figure 4.11 Effect of superficial gas velocity on solids shear stress profile

Figure 4.11 Effect of superficial gas velocity on solids shear stress profile
(air-water-glass beads $150 \mu \mathrm{m}$ ) in 6" column with $9.1 \%$ vol. solids loading at $0.1 \mathrm{MPa}$

Figure 4.12 Effect of superficial gas velocity on TKE (air-water-glass beads $150 \mu \mathrm{m}$ ) in 6" column with $9.1 \%$ vol. solids loading at $0.1 \mathrm{MPa}$

Figure 4.13 Effect of superficial gas velocity on a) solids axial diffusivity, and b) solids radial diffusivity (air-water-glass beads $150 \mu \mathrm{m}$ ) in 6 " column with $9.1 \%$ vol. Solids loading at $0.1 \mathrm{MPa}$

Figure 4.14 Effect of operating pressure on gas holdup radial profile using air-water-glass beads $(150 \mu \mathrm{m})$ in 6 " column with $9.1 \%$ vol. solids loading at $45 \mathrm{~cm} / \mathrm{s}$

Figure 4.15 Effect of operating pressure on axial velocity profile using airwater-glass beads $(150 \mu \mathrm{m})$ in $6 "$ column with $9.1 \%$ vol. solids loading at $45 \mathrm{~cm} / \mathrm{s}$

Figure 4.16 Effect of operating pressure on solids shear stress profile using air-water-glass beads $(150 \mu \mathrm{m})$ in 6 " column with $9.1 \%$ vol. solids loading at $45 \mathrm{~cm} / \mathrm{s}$

Figure 4.17 Effect of operating pressure on solids TKE using air-waterglass beads $(150 \mu \mathrm{m})$ in $6 "$ column with $9.1 \%$ vol. solids loading at $8 \mathrm{~cm} / \mathrm{s}$

Figure 4.18 Effect of operating pressure on a) solids axial diffusivity profile b) solids radial diffusivity profile using air-water-glass beads $(150 \mu \mathrm{m})$ in 6 " column with $9.1 \%$ vol. solids loading at $8 \mathrm{~cm} / \mathrm{s}$

Figure 4.19 Effect of superficial gas velocity on gas holdup profile (airwater) in 6" column at $0.1 \mathrm{MPa}$

Figure 4.20 Effect of superficial gas velocity on liquid axial velocity profile (air-water) in 6" column at $0.1 \mathrm{MPa}$

Figure 4.21 Effect of superficial gas velocity on liquid shear stress profile (air-water) in 6" column at $0.1 \mathrm{MPa}$

Figure 4.22 Effect of superficial gas velocity on TKE (air-water) in 6" column at $0.1 \mathrm{MPa}$

Figure 4.23 Effect of superficial gas velocity on a) liquid axial diffusivity, and b) liquid radial diffusivity (air-water) in 6" column at 0.1 $\mathrm{MPa}$

Figure 4.24 Effect of operating pressure on gas holdup radial profile using air-water system in 6 " column with at $45 \mathrm{~cm} / \mathrm{s}$ 
Figure 4.25 Effect of operating pressure on liquid axial velocity profile using air-water system in 6" column at $45 \mathrm{~cm} / \mathrm{s}$

Figure 4.26 Effect of operating pressure on shear stress profile using airwater system in 6" column at $45 \mathrm{~cm} / \mathrm{s}$

Figure 4.27 Effect of operating pressure on TKE using air-water system in 6 " column at $45 \mathrm{~cm} / \mathrm{s}$

Figure 4.28 Effect of operating pressure on a) axial diffusivity profile b) radial diffusivity profile using air-water system in 6" column at $45 \mathrm{~cm} / \mathrm{s}$

Figure 4.29 Effect of superficial gas velocity on a) gas holdup, and b) solids holdup profile (air-Therminol LT-glass beads) in 6" column with $9.1 \%$ vol. solids loading at $0.1 \mathrm{MPa}$.

Figure 4.30 Effect of operating pressure on a) gas holdup, and b) solids holdup profile (air-Therminol LT-glass beads) in 6" column with $9.1 \%$ vol. solids loading at $14 \mathrm{~cm} / \mathrm{s}$. 


\title{
ADVANCED DIAGNOSTIC TECHNIQUES FOR THREE-PHASE SLURRY BUBBLE COLUMN REACTORS (SBCR)
}

\author{
Final Technical Report \\ DE-FG-26-99FT40594
}

\section{EXECUTIVE SUMMARY}

The objectives set for this cooperative research effort between Washington University (WU), Ohio State University (OSU), and Air Products and Chemicals Inc. (APCI) is to advance the understanding of the hydrodynamics of Fischer-Tropsch (FT) Slurry Bubble Column Reactors (SBCR) via advanced diagnostics techniques have been achieved successfully despite the unexpected challenging technical problems which were all resolved in a systematic manner.

This report summarizes the accomplishments made and discusses the findings obtained during the four years of this project period that include the no-cost extension year (the fourth year). The report is organized in individual sections as follows.

Section 1 provides a review of the objectives and tasks set for the project, list of accomplishments during the first, second, third year, and the no-extension period (i.e. fourth year).

Section 2 summarizes the technical review made. However, the detailed review of the aspects of high pressure phenomena of bubbles in liquids and liquid-solids suspension is discussed in Appendix A. This includes bubble dynamics, and macroscopic hydrodynamics of bubble column and slurry bubble column reactors.

Section 3 describes in detail the experimental facilities at Washington University and Ohio State University and the advanced techniques like Fiber Optic Probe, Laser Doppler Anemometry (LDA), Pressure Drop fluctuation, Dynamic Gas Disengagement (DGD), Computer Automated Radioactive Particle Tracking (CARPT), Computed Tomography (CT) used to study hydrodynamics of high pressure slurry bubble column. The unexpected challenging technical difficulties encountered during CARPT/CT implementation in high pressure slurry bubble column are outlined and discussed.

Section 4 discusses the results of the performed experiments at Ohio State University and Washington University. This includes discussing and analyzing the effects of reactor pressure, superficial gas velocity, solids loading, and liquid physical properties on the overall gas holdup, holdups distribution, recirculation velocity, turbulent parameters, and bubble dynamics. 
The details of Section 5, 6, 7, 8, 9, and 10 are attached as Appendix A, B, C, D, E, and F, respectively.

Appendix A comprises review of the aspects of high pressure phenomena of bubbles in liquids and liquid-solids suspension.

Appendix B discusses the development of correlations for radial gas holdup profile and axial liquid velocity profile.

Appendix C provides details for the development of a correlation proposed to predict overall gas holdup in bubble columns over a wide range of conditions using Artificial Neural network.

Appendix D discusses the development of a new correlation to predict gas-liquid mass transfer coefficient at high pressure operation based on high pressure gas holdup and atmospheric data of volumetric mass transfer coefficient.

Appendix E discusses the development of CT/Overall gas holdup methodology developed to calculate phase holdups distribution of three dynamic phases using single source $\gamma$-ray CT. The effects of superficial gas velocity, operating pressure, and physical property has been studied using the developed methodology.

Appendix $\mathrm{F}$ provides the details of problems encountered during the implementation of $\mathrm{CARPT} / \mathrm{CT}$ on high pressure stainless steel bubble column and also the details of how these problems have been resolved. 


\section{OVERALL OBJECTIVES}

The overall objective of this project is to quantify the hydrodynamics of FT slurry bubble column reactor (SBCR) by utilizing advanced diagnostic techniques. This can be achieved by properly describing the distribution of phases and liquid (slurry) circulation and turbulence in SBCR for Fischer-Tropsch (FT) synthesis via studying the microstructure of the gas-liquid-solid mixtures in a comparable fluid to FT waxes in 2 inch diameter column, developing a fundamental understanding as to how important the physical and fluid dynamic properties can be "finger-printed" via various diagnostic techniques such as laser doppler anemometry (LDA), optical probe and differential pressure fluctuation technique and by measuring large scale hydrodynamic parameters at high pressure and high gas velocity in a 6 inch diameter slurry bubble column using computed tomography (CT) and computer automated radioactive particle tracking (CARPT). CARPT and CT are the only non-invasive techniques that can provide information on slurry velocity and density profiles in 3D domain. Such data provides a firm scientific and engineering basis for scale-up and design of FT SBCR. In addition, the obtained results can be utilized as a benchmark to validate the computational fluid dynamic codes.

This grant enables a unique integration of the expertise of the two universities (Washington University, WU and Ohio State University, OSU) and industry (Air Products and Chemicals, APCI) towards achieving the goals set for the project. This study complements well the work performed at WU, OSU, Iowa State University (ISU) and Sandia National Laboratory, Contract No. DE-FC-22-95PC95051, related to the La Porte Advanced Fuels Demonstration Unit (AFDU) operated by Air Products and Chemical Inc. with the Department of Energy funding which focused on advancing the state-of-art in understanding the fluid dynamics of slurry bubble columns and replacing empirical design methods with a more rational approach.

The goals set for this project are as follows:

TASK 1: $\quad$ Literature Review

Physicochemical properties and their effect on the hydrodynamics of bubble columns.

- $\quad$ Models used to predict FT reactor performance.

TASK 2: $\quad$ Based on Task 1, identify the range of intrinsic properties (density, viscosity and surface tension) of the fluids used for the FT synthesis. Identify a solvent that, at room temperature and pressure up to 200 psig, will mimic the hydrodynamics of FT wax (at FT reaction conditions).

- $\quad$ Identify the particle type and size to be used.

TASK 3: $\quad$ Using the identified system (solvent-particle-air), perform the following investigation on the hydrodynamics in a 2" diameter column:

Investigate the effect of reactor pressure on the flow field and turbulent parameters using Laser Doppler Anemometer (LDA).

- $\quad$ Identify the flow regime transition and investigate the effect of reactor pressure on the flow regime transition using $\Delta \mathrm{P}$ fluctuation measurements. 
- $\quad$ Measure overall gas holdup using change in slurry height.

- $\quad$ Measure bubble size and bubble rise velocity using Optical Probe.

TASK 4: $\quad$ Using air-water-glass beads, air-water, and the identified system in Task 2 or a system with similar properties, investigate the hydrodynamics in a 6 " diameter column via CT and CARPT techniques. The following will be measured:

- $\quad$ Phase distribution profiles using CT

- $\quad$ Flow field and turbulent parameters using CARPT

- $\quad$ Gas holdup using CT and change in slurry height.

TASK 5: Develop needed correlations for improved scale-up procedure for slurry bubble column.

TASK 6: $\quad$ Prepare final report

\subsection{Accomplishments During the First Year}

The first year was dedicated for the preparation of the technical review, experimental facilities and the advanced measurement techniques. A new correlation was developed to predict the liquid-solid mass transfer coefficient in high pressure bubble column based on the atmospheric pressure data. The accomplishments were as follows:

$>$ The technical review of the variables affecting SBCR performance, some aspects of bubble dynamics and hydrodynamic properties and the physical properties of FT waxes and catalyst were performed.

$>$ The experimental facilities and the advanced measurement techniques were prepared. The preparation includes the following units:

- High pressure (up to $3000 \mathrm{psi}$ ) and high temperature (up to $250^{\circ} \mathrm{C}$ ) 2-inch diameter slurry bubble column set-up.

- High pressure (up to 200 psi) 6-inch diameter slurry bubble column set-up.

- Two facilities will be used; one for computer automated radioactive particle tracking (CARPT) and computed tomography (CT) techniques and another one for pressure drop measurements. The later facility consists of a 6 -inch diameter column equipped with 6- windows and 15 ports along the column.

- Laser Doppler Anemometry (LDA) for 2" slurry bubble column facility.

- CARPT and CT for 6-inch slurry bubble column facility.

- Techniques to measure in situ the intrinsic density, viscosity and surface tension of the liquid-phase that will be used for hydrodynamics investigation which mimic the hydrodynamics of FT waxes.

The solvents that mimic FT waxes at FT operating conditions were identified and the gas and solid phases to be used in the hydrodynamics investigation were selected.

$>$ A new correlation to estimate the mass transfer coefficient at high pressure based on atmospheric pressure data was developed.

\subsection{Accomplishments During the Second Year}

Experimental investigation of the hydrodynamics of Norpar 15- nitrogen-glass beads in 2" column using LDA/pressure drop/slurry height measurements was executed. 
The unexpected technical challenging difficulties encountered during CARPT/CT implementation on high pressure stainless steel SBCR were resolved.

$>$ Experimental investigations of the effect of reactor pressure and gas flow rate on the hydrodynamics of air-water-glass beads $(150 \mu \mathrm{m})$ system in 6" column using CT/CARPT were performed.

$>$ Correlations to predict radial gas holdup and axial liquid recirculation velocity profiles needed for scale-up of bubble columns were developed.

\subsection{Accomplishments During the Third Year}

$>$ Completion of data processing and analysis of CARPT experiments in 6" column for air-water-glass beads $(150 \mu \mathrm{m})$ system was achieved.

$>$ Comparison of the proposed CARPT/CT/differential pressure measurements (DP) and CT/Overall Gas holdup methodology to compute three phases distribution in airwater-glass beads $(150 \mu \mathrm{m})$ system in 6" column was performed. The data processing and analysis were completed using the developed CT/Overall Gas holdup methodology.

$>$ Experimental investigations of hydrodynamics of air-Therminol LT-glass beads $(150 \mu \mathrm{m})$ system in 6" column were executed using CT. The data processing and analysis of CT experiments were performed using the developed CT/Overall Gas holdup methodology.

$>$ Experimental investigations of hydrodynamics of air-Norpar 15-glass beads (150 $\mu \mathrm{m})$ in 2" column using Dynamic Gas Disengagement/Pressure Drop fluctuations/Fiber Optic Probe measurements were performed and completed.

$>$ Artificial Neural Network based correlation for prediction of overall gas holdup in bubble column reactors needed for their design and scale-up was developed.

\subsection{Accomplishments During the no-cost extension (Fourth) Year}

$>$ The developed radioactive $150 \mu \mathrm{m}$ Scandium particles for CARPT experiments that match the size and density of the $150 \mu \mathrm{m}$ glass beads used as solids in the performed experiments were irradiated at Oak Ridge National Laboratory after trial and error attempts and systematic investigation. During the third year and the no-cost extension (fourth) year, the reactor was shut down. Therefore, we had to repeat the same effort of trial and error and systematic investigation to irradiate the particles at MIT nuclear reactor. After many attempts during the third year and fourth year, we were able to irradiate a Scandium particle suitable for air-water system (i.e. match the density of water). Therefore, this system was investigated and the results are reported in this report for the fourth year.

$>$ Experimental investigations of the hydrodynamics of air-water system at high pressure and high superficial gas velocity were performed using CARPT/CT.

$>$ The effects of reactor pressure, superficial gas velocity, and liquid physical properties on the gas and solids holdups distributions were analyzed and discussed.

$>$ The final report has been prepared. 


\section{TECHNICAL REVIEW}

\subsection{Variables Affecting Slurry Bubble Column (SBCR) Performance}

A slurry bubble column reactor (SBCR) is usually a cylindrical vessel in which gas (containing one or more reactants, e.g. synthesis gas for FT processes) is sparged through the liquid (containing liquid reactant(s) and products), and a finely dispersed catalyst. As long as the operating liquid superficial velocity (in the range of 0 to $2 \mathrm{~cm} / \mathrm{s}$ ) is an order of magnitude smaller than the superficial velocity of the gas $(1$ to $30 \mathrm{~cm} / \mathrm{s})$, and the catalyst particles are small (less than $50 \mu \mathrm{m}$ ) and not excessively heavy, the gas dominates the hydrodynamics and, by buoyancy forces resulting from the nonuniform cross-sectional gas holdup distribution induces liquid velocities order of magnitude larger than the liquid superficial velocity. The finely dispersed catalyst follows the motion of the liquid.

Interpretation of SBCR performance must rely on an appropriate model which properly accounts for the events on the molecular scale (e.g. kinetics and catalyst particle performance, etc.), micro-scale (e.g. transport of reactants and products to and from the catalyst particle and of reactants from gas bubbles to the liquid) and macro-scale (e.g. liquid backmixing) on heat transfer and reactor performance. We know that numerous design and operating variables, listed in Figure 2.1, and physicochemical and thermodynamic properties of the fluid affect the many highly interactive phenomena in SBCR. All of these in turn affect reactor performance.

A slurry bubble column reactor for FT synthesis and other syngas processes in order to be economically successful, must operate at high volumetric productivity which requires high activity catalyst, high catalyst loading of the slurry, large gas flow rate and high gas conversion. The ability to achieve complete catalyst suspension and the desired flow pattern (degree of backmixing) of the liquid phase are crucial to the targeted reactor performance. In order to accomplish these an improved understanding and quantification of the key hydrodynamic phenomena is required.

We focus here our attention on properly describing the liquid (slurry) circulation and turbulence in SBCR for Fischer Tropsch synthesis because it is the liquid mixing that affects catalyst distribution, bubble coalescence, gas-liquid interfacial area, mass transfer coefficients and heat transfer from the reactor. We first summarize our current understanding of flow regimes and gas holdup, and their effect on liquid backmixing.

Flow regime affects gas holdup and holdup distribution. The available standard flow maps (e.g. Shah et al., 1982) are old and not very reliable in identifying the flow regime in FT SBCRs. It is commonly assumed, based on evidence of multiple bubble sizes (Patel et al., 1990; De Swart, 1996), that churn-turbulent flow occurs in FT waxes at superficial gas velocities above $5 \mathrm{~cm} / \mathrm{s}$. This needs additional verification. Information on characteristic bubble size as well as reliable diagnostic of the flow regime are needed.

Gas holdup is the fraction of the column occupied by gas. As Fan (1989) illustrates, existing older correlations cannot predict holdup accurately and no agreement regarding holdup is reached for Fischer Tropsch waxes (Quicker and Deckwer, 1981). Kemoun et al. (2001) studied the effect of pressure on gas holdup and its cross-sectional distribution using $\gamma$-ray Computed Tomography (CT) in air-water system at elevated pressures up to 
0.7 MPa. The cross-sectional average gas holdup was calculated using the collected data and compared with various correlations found in the literature. Their main findings are:

- At atmospheric pressure, the correlation of Idogawa et al. (1985) was in the best agreement with experimental data except for $U_{G}=0.05 \mathrm{~m} / \mathrm{s}$. This operating condition is near the transition point, and the correlation and data may not belong to the same flow regime.

- At higher pressures and over the entire superficial gas velocity range investigated (2 to $30 \mathrm{~cm} / \mathrm{s}$ ), the correlation of Hammer et al. (1984) gives the best prediction of gas holdup data (average error of 12-17\%) followed by Wilkinson et al. (1992; average error of 14-18\%) and Idogawa et al. (1987; average error of 18-20\%).

- At higher pressures and high superficial gas velocity $\left(U_{G}>0.1 \mathrm{~m} / \mathrm{s}\right)$, in addition to the correlations of Idogawa et al. (1987), and Hammer et al. (1984), the correlation of Krishna et al. (1996) and Luo et al. (1999) also seem to provide reasonable predictions of the measured gas holdup.

They also concluded that they were not able to find any correlation that consistently predicted their experimental data well under all process conditions, which indicates the need for better characterization of the levels of liquid recirculation and turbulence which are needed for development of a more fundamentally based model for prediction of gas holdups (Fan, 1989).

Radial gas holdup distribution drives liquid recirculation as originally shown by Hills (1974). This distribution depends on operating conditions, physical properties of the system and distributor type (e.g. Chen et al., 1999; Rice and Geary, 1990). It has not been determined in FT SBCRs.

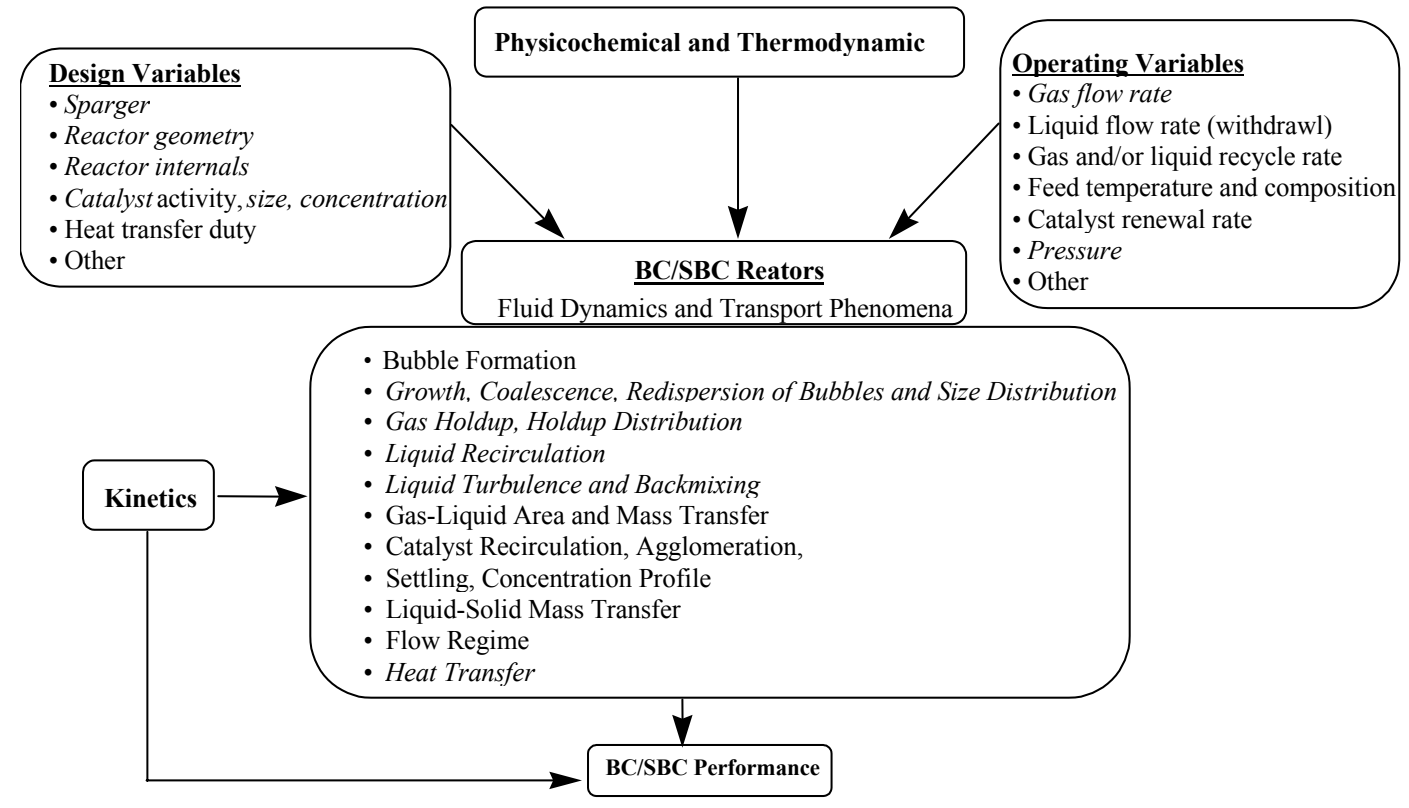

Figure 2.1: Variables that affect SBCR performance 
Quantification of liquid backmixing is essential for reactor design, scale-up and interpretation of reactor performance. This is most commonly done by the use of the axial dispersion model. Fan (1989) compares the predictions for the liquid axial dispersion coefficient obtained from various correlations at different gas velocities and in columns of different diameter for the air-water system. The agreement leaves a lot to be desired, which is not surprising since the model assumes a flat velocity profile with eddy axial dispersion superimposed on it. However, the axial liquid velocity profile in a bubble column is anything but flat and, therefore, the axial dispersion coefficient has to account for a variety of mixing mechanisms, including non-uniform velocity, convective transport, bubble wake turbulence, liquid axial and radial turbulence, etc. The dominant mixing mechanism is due to liquid recirculation with contribution from wake and eddy dispersion. Therefore, the correlations reported in the literature, and summarized by Fan (1989), form a poor basis for design/scale-up.

Recently, Degaleesan (1997) has shown that the effective axial dispersion coefficient can be related to liquid recirculation and radial eddy diffusivity via Taylor type diffusivity and to the axial eddy diffusivity. She proposed a scale-up procedure to estimate the fluid dynamic parameters of industrial systems needed for prediction of their performance. The validity of the model proposed by Degaleesan (1997) for evaluation of these parameters on FT systems needs experimental verification.

Liquid recirculation in bubble columns (BC) has been observed and documented by Hills (1974), Rice and Geary (1990) and Devanathan (1991). A novel noninvasive experimental technique, based on monitoring the motion of a single radioactive particle, was used to demonstrate (Devanathan, 1991; Degaleesan 1997) that multiple stationary circulation cells, as proposed by Joshi and Sharma (1979), do not exist. Some preliminary data on slurry recirculation has been reported by Sannaes et al. (1995), Grevskott et al. (1996) and Rados (1999). Additional data in more concentrated slurries is needed.

Mean bubble size and bubble size distribution are also important parameters for SBCR performance, which depends on the flow regime and properties of the system. Insufficient information exists for SBCR in general and for FT systems in particular in spite of the recent work by de Swaart (1996).

\subsection{Some aspects of bubble dynamics and hydrodynamic properties}

The critical review of high-pressure phenomena of bubbles in liquid and liquid-solid suspension is discussed in Appendix A attached (Fan, et al., 1999). In this section some aspects of bubble dynamics and hydrodynamic properties that are reported in Appendix A are summarized.

\subsubsection{Bubble Dynamics}

\section{Single bubble rise velocity}

In liquid-solid suspensions under elevated pressure and temperature conditions, the bubble rise velocity is discussed in light of both the apparent homogeneous (or effective) properties of the suspension and the recently evolved numerical prediction based on a computational model for gas-liquid-solid fluidization systems. In the literature, it is found 
that the single bubble rise velocity does not depend on the gas density over the range of 0.1 to $30 \mathrm{~kg} / \mathrm{m}^{3}$. The effects of pressure and temperature, or more directly, the effects of physical properties of the gas and liquid phases on the variation of bubble rise velocity $\left(u_{b}\right)$ with bubble diameter $\left(d_{b}\right)$ could be represented or predicted most generally by the Fan-Tsuchiya equation (Fan and Tsuchiya, 1990) among three predictive equations. The other two are the modified Mendelson's wave-analogy equation (Mendelson, 1967) and a correlation proposed by Tomiyama et al. (1995).

In general, the bubble rise velocity decreases with an increase in pressure for a given solids holdup. A more drastic reduction in $u_{b}$ can arise from the addition of solid particles. While the particle effect is small at low solids holdup, the effect is appreciable at high solids holdup, especially for high liquid viscosity. The reduction of the bubble rise velocity with an increase in pressure can lead to a significant increase in the gas holdup of three-phase fluidized beds. By comparing the pressure effect on the gas holdup with that on the bubble rise velocity, the increase in gas holdup with pressure is a consequence of the decreases in both the bubble size and the bubble rise velocity.

\section{Heterogeneous approach: Discrete-phase computation}

A two-dimensional discrete-phase simulation model for gas-liquid-solid fluidization systems has been developed recently (Jean and Fan, 1990; Luo, et al., 1997; Zhang et al., 1998a,b) to provide a much more thorough scheme of prediction of a single bubble rising in a liquid-solid fluidized bed. In this model, the volume-averaged method, the dispersed particle method (DPM) and the volume-of-fluid (VOF) method are used to account for the flow of liquid, solid, and gas phases, respectively. A bubble induced force (BIF) model, a continuum surface force (CSF) model, and Newton's third law are applied to account for the couplings of particle-bubble (gas), gas-liquid, and particle-liquid interactions, respectively. A close distance interaction (CDI) model is included in the particle-particle collision analysis, which considers the liquid interstitial effects between colliding particles.

The motion of a particle in a flow field can be described in Lagrangian coordinates with its origin attached to the center of the moving particle. The motion of a single particle can be described by its acceleration and rotation in a nonuniform flow field. The forces acting on a particle include interface forces between the fluid and particle, and forces imposed by external fields. The total force acting on a particle is composed of all applicable forces, including drag, added mass, gravity/buoyancy, Magnus force, Basset force, and other forces. The general scheme of a stepwise molecular dynamic (MD) simulation (Tildesley, 1987), based on a predictor-corrector algorithm, is used to compute the particle motion. The hard sphere approach is used for the collision dynamics. The collinear collision model is used to determine the normal velocity and momentum changes of colliding particles. The model includes the detailed close-range particle-fluid and particle-particle interactions during the entire process of particle collision. The tangential velocity and momentum changes are formulated and calculated based on a sticking/sliding model (Zhang et al., 1998a). 


\section{Bubble formation, initial bubble size, and jetting}

Among various factors that affect the bubble formation, the wettability of the orifice surface is an important factor, which affects the initial size of the bubble formed on the orifice. It is found that initial bubble size increases significantly with the contact angle between the bubble and the orifice surface when the contact angle exceeds the threshold value of $45^{\circ}$. The high-pressure studies indicated that an increase in gas density reduces the size of bubbles formed from a single orifice. A mechanistic model is described to predict the initial bubble size in liquid-solid suspensions at high-pressure conditions (Luo, et al., 1998c). The model considers various forces induced by the particles. During the expansion and detachment stages, particles collide with the bubble and stay on the liquid film. The particles and the liquid surrounding the bubble are set in motion as the bubble grows and rises. The model is applied to simulate the bubble formation process under constant flow conditions, which are characterized by constant gas flow rate through the orifice. When the volume of the gas chamber is small, the bubble formation can normally be assumed under constant flow conditions.

\section{Bubble coalescence}

For gas-liquid systems, the experimental results available in the literature indicate that an increase of pressure retards the bubble coalescence. It is known that surface tension decreases and liquid viscosity increases with increasing pressure. In addition, particle sphericity $\phi$ increases with pressure. These variations contribute to the reduction of the film thinning velocity, and hence, the bubble coalescence rate, as pressure increases. As a result, the time required for two bubbles to coalesce is longer and hence the rate of overall bubble coalescence in the bed is reduced at high pressures. Moreover, the frequency of bubble collision decreases with increasing pressure. An important mechanism for bubble collision is bubble wake effects (Fan and Tsuchiya, 1990). When the differences in bubble size and bubble rise velocity are small at high pressures, the likelihood of small bubbles being caught and trapped by the wakes of large bubbles decreases. Therefore, bubble coalescence is suppressed by the increase in pressure, due to the longer bubble coalescence time and the smaller bubble collision frequency.

\section{Bubble breakup and maximum stable bubble size}

There are many models proposed in the past that predicts the maximum stable bubbles size but they all do not account for the internal circulation of the gas. The internal circulation velocity is of the same order of magnitude as the bubble rise velocity. A centrifugal force is induced by this circulation, pointing outwards toward the bubble surface. This force can suppress the disturbances at the gas-liquid interface and thereby stabilizing the interface. On the other hand, the centrifugal force can also disintegrate the bubble, as it increases with an increase in bubble size. The bubble breaks up when the centrifugal force exceeds the surface tension force, especially at high pressures when gas density is high. 
An analytical criterion for the bubble breakup is derived by considering a single large bubble rising in a stagnant liquid or slurry at a velocity of $u_{b}$, without any disturbances on the gas-liquid interface. The bubble is subjected to breakup when its size exceeds the maximum stable bubble size due to the circulation-induced centrifugal force (Luo et al., 1998a).

\section{Macroscopic Hydrodynamics}

\section{Hydrodynamic similarity}

Numerous studies have been conducted to investigate the effect of pressure on the gas holdup of bubble columns and three-phase fluidized beds. Further, empirical correlations have been proposed for gas holdup in bubble columns operated at elevated pressure and temperature (Fan et al., 1999; Kemoun et al., 2001). A similarity rule has been developed to simulate the hydrodynamics of industrial reactors which requires hydrodynamic

similarity of the following dimensionless groups to be the same: $U_{g} / u_{\max }, M o_{m}$, and $\rho_{g} / \rho_{m}$.

\section{Heat transfer characteristics}

A consecutive film and surface renewal model is used to analyze the heat transfer behavior. The model assumes that a thin liquid film exists surrounding the heating surface; and liquid elements are forced to contact the outer surface of the film, due to the passage of bubbles. The liquid elements contact the film for a short time, $t_{c}$, and then, are replaced by fresh liquid elements. The heat is transferred to the bulk liquid through conduction by the liquid film and unsteady state conduction by the liquid elements. The heat transfer coefficient is expressed in terms of the physical properties of the liquid, the film thickness, and the contact time of the liquid elements (Wason and Aluwalia, 1969). By considering the pressure effects on the physical properties of liquid and bubble characteristics, such as bubble size and bubble rise velocity, this model may be used to analyze the heat transfer behavior in a high-pressure system.

\subsection{Models used for FT reactor performance prediction}

The use of slurry bubble column reactors (SBCR) provides an attractive alternative to traditional vapor-phase processes. Fischer-Tropsch (FT) synthesis has been recognized as a promising method for environmentally benign, indirect, coal utilization. Advantages of the three phase slurry FT synthesis process include: nearly isothermal operation, high catalyst effectiveness factor due to the smaller catalyst particle sizes, lower rate of catalyst deactivation and low pressure drop. Hence, SBCR is the favored reactor for commercialization of FT synthesis. However, one of the disadvantages of FT SBCR is the uncertainty of its design and scale-up in addition to the need for liquid-catalyst separation, catalyst attrition, etc. In addition to the mentioned advantages, slurry FT synthesis process doesn't require high hydrogen/carbon monoxide ratio syngas compared to fluidized or fixed bed reactor processes and hence, it can use the low hydrogen/carbon 
monoxide ratio syngas that is produced by the new generation of coal gasifiers. The chemical reaction of the FT synthesis (Paraffin synthesis) is as follows:

$$
\mathrm{CO}+\left(1+\frac{m}{2 n}\right) \mathrm{H}_{2} \longrightarrow \frac{1}{n} \mathrm{C}_{n} \mathrm{H}_{m}+\mathrm{H}_{2} \mathrm{O} \quad, m=2 n+2
$$

In processes that utilize iron catalyst and/or low hydrogen/carbon monoxide inlet ratio water gas shift reaction (WGS) should also be considered (Stern et al., 1985, Prakash, 1993; van der Laan et al., 1999):

$$
\mathrm{CO}+\mathrm{H}_{2} \mathrm{O} \longrightarrow \mathrm{CO}_{2}+\mathrm{H}_{2}
$$

Detailed kinetics that is probably needed for the accurate FT process modeling should at least also include methane $(n=1)$, olefin $(m=2 n)$ synthesis and Boudouard's reaction:

$$
2 \mathrm{CO} \longrightarrow \mathrm{C}_{(s)}+\mathrm{CO}_{2}
$$

The basic paraffin synthesis kinetics (i.e., FT synthesis kinetics) have been almost exclusively used in the published FT models. Paraffin synthesis kinetics have been found to be of a Langmuir-Hinshelwood (L-H) type (Van der Laan, et al., 1999). However at hydrogen conversion below $60 \%$ first order hydrogen kinetics (FTS $1^{\text {st }}$ ) has been shown to be a reasonable approximation (Dry, 1976; Huff and Satterfield, 1984). Table 2.1 lists the kinetic types used in FT SBCR performance modeling.

Due to the lack of understanding of the hydrodynamics of churn-turbulent slurry bubble column, most of the models assumed uniform concentration of the catalyst throughout the reactor. Following the work of Kato et al. (1972) some of the models calculated the catalyst axial concentration profile by using sedimentation and dispersion model (SDM) (Deckwer et al., 1982; Mills et al., 1996). These models have shown that in spite of small particle size and churn turbulent regime solids phase concentration is the highest at the bottom of the column and exponentially drops with height for batch slurry operation. However, the profiles of solids distribution in co-current and counter-current modes of operation strongly depend on the direction and magnitude of slurry inlet velocity (Mills et al., 1996).

Fisher-Tropsch process is highly exothermic and hence, one would naturally consider modeling the energy balance. However, results of several models (Deckwer et al., 1982; Mills et al., 1996) predict nearly isothermal operation of the FT slurry reactor. Turner and Mill's (1990) model predicted a slight temperature axial gradient (with local temperature within $20 \%$ from wall temperature). They related it to catalyst concentration axial profiles (SDM). In the mentioned models, the energy balance axial convection, reaction heat, wall convection and axial dispersion are included. However, all of these models neglected latent heat of evaporation whose contribution may be expected to be appreciable.

All published models on FT process treated the solids and liquid as one pseudo homogeneous slurry phase (SL). Slurry phase is the most often modeled as completely mixed (CM) (Bukur, 1983; Maretto and Krishna, 1999; van der Laan, 1999) or using axial dispersion model (ADM) (Deckwer et al., 1982; Mills et al., 1996; De Swart et al., 1997). Leib et al. (1995) used multi cell model for both liquid (completely mixed) and 
gas phase (plug flow, PF) so that the extent of backmixing has been varied by changing the number of mixing cells. Turner and Mills (1990) compared the predictions of mixing cell model (MCM) and axial dispersion model (ADM) and concluded that mixing cell model is more realistic approach although both models predicted the same performance of FT slurry reactor when number of cells (MCM) and Peclet number (ADM) were matched using a proposed correlation. Gas phase $(\mathrm{G})$ has been traditionally modeled as a single phase in plug flow (PF) or with axial dispersion. Last few years several models appeared that described gas phase using two bubble class approach. In this approach small bubbles phase (SB) is modeled as completely mixed (as slurry phase), while large bubbles phase (LB) is in plug flow (Maretto and Krishna, 1999; van der Laan et al., 1999), or alternatively, both of the gas phases are modeled using ADM (De Swart et al., 1997).

Table 2.1 shows a summary of the most relevant studies on the modeling of FT slurry bubble column reactors. The stoichiometry of the Fischer-Tropsch synthesis (eq. 2.1) is such that more moles reacts than it is produced. Because of this the amount of gas phase decreases along the FT slurry reactor with experimentally determined contraction factor $\alpha$ of about -0.5 . Because of this effect valid model of the FT process must include a closure for the superficial gas velocity (SGV) axial profile. Classical approach assumes linear relationship between SGV and conversion of syngas (Deckwer et al., 1982):

$U_{G}=U_{G}^{i}\left(1+\alpha X_{H_{2}+C O}\right)$

Overwhelming number of different models is adopting this relationship (Table 2.1) although strictly this is valid only in steady state when axial mixing and convection in the liquid phase can be neglected (Stern et al., 1985). More accurate, rigorous modeling of the SGV axial profile is based on the overall gas phase mass balance (Prakash, 1993; Stern et al., 1985), which requires knowledge of the concentration profiles of all gaseous species. The drawback of this approach is obviously larger number of equations that have to be solved compared to classical Deckwer's approach.

Model for the prediction of the Fischer-Tropsch slurry reactor performance is needed which properly should include detailed kinetics with all relevant species, mixing pattern of different phases (e.g., the phenomenological multi cell recirculation model (Degaleesan et al., 1997)), change in SGV, solids axial profile, (e.g., the mechanistic model (Murray and Fan, 1989)), etc. In addition the present energy balance modeling may need to be improved by accounting for the latent heat of evaporation. In order to achieve this, detailed understanding of the hydrodynamics of FT slurry bubble column reactor via advanced diagnostic techniques is needed which is the focus of the tasks set for this grant. 
Table 2.1. Summary of models for prediction of the Fischer-Tropsch process performance.

\begin{tabular}{|c|c|c|c|c|c|c|c|}
\hline Model & Kinetics & $\begin{array}{l}\text { Accounted } \\
\text { species }\end{array}$ & $\begin{array}{l}\text { Phase } \\
\text { degree of } \\
\text { mixing }\end{array}$ & $\begin{array}{l}\text { Gas } \\
\text { velocity } \\
\text { profile }\end{array}$ & $\begin{array}{l}\text { Solids } \\
\text { profile }\end{array}$ & $\begin{array}{l}\text { Energy } \\
\text { balance }\end{array}$ & $\begin{array}{l}\text { Steady } \\
\text { state } \\
\text { model }\end{array}$ \\
\hline $\begin{array}{l}\text { van der Laan et } \\
\text { al., } 1999\end{array}$ & $\begin{array}{l}\text { FTS, L-H } \\
\text { WGS, L-H }\end{array}$ & \begin{tabular}{|l|}
$\mathrm{H}_{2}, \mathrm{CO}$ \\
$\mathrm{H}_{2} \mathrm{O}, \mathrm{CO}_{2}$ \\
n products \\
\end{tabular} & $\begin{array}{l}\text { SB-CM } \\
\text { LB-PF } \\
\text { SL-CM } \\
\end{array}$ & $f(X, \alpha)$ & uniform & Yes & Yes \\
\hline $\begin{array}{l}\text { Maretto and } \\
\text { Krishna, } 1999\end{array}$ & FTS, L-H & $\mathrm{H}_{2}, \mathrm{CO}$ & $\begin{array}{l}\text { SB-CM } \\
\text { LB-PF } \\
\text { SL-CM }\end{array}$ & uniform & uniform & $\begin{array}{l}\text { iso- } \\
\text { thermal }\end{array}$ & Yes \\
\hline $\begin{array}{l}\text { De Swart et al., } \\
1997\end{array}$ & FTS, $1^{\text {st }}$ & $\mathrm{H}_{2}$ & $\begin{array}{l}\text { SB-AD } \\
\text { LB-AD } \\
\text { SL-AD }\end{array}$ & $f(X, \alpha)$ & SDM & Yes & dynamic \\
\hline $\begin{array}{l}\text { Mills et al., } \\
1996\end{array}$ & FTS, $1^{\text {st }}$ & $\mathrm{H}_{2}$ & $\begin{array}{l}\text { G-AD } \\
\text { SL-AD }\end{array}$ & $f(X, \alpha)$ & SDM & Yes & Yes \\
\hline Leib et al., 1995 & FTS, $1^{\text {st }}$ & $\mathrm{H}_{2}$ & $\begin{array}{l}\text { G-nCM or } \\
\text { nPF } \\
\text { L-nCM }\end{array}$ & $f(X, \alpha)$ & uniform & $\begin{array}{l}\text { iso- } \\
\text { thermal }\end{array}$ & Yes \\
\hline Prakash, 1993 & $\begin{array}{l}\text { FTS, L-H } \\
\text { WGS, L-H }\end{array}$ & $\begin{array}{l}\mathrm{H}_{2}, \mathrm{CO}, \\
\mathrm{H}_{2} \mathrm{O}, \mathrm{CO}_{2}\end{array}$ & $\begin{array}{l}\text { G-AD } \\
\text { SL-AD }\end{array}$ & $\begin{array}{l}\text { overall } \\
\text { gas MB }\end{array}$ & SDM & Yes & Yes \\
\hline $\begin{array}{l}\text { Truner and } \\
\text { Mills, } 1990\end{array}$ & FTS, $1^{\text {st }}$ & $\mathrm{H}_{2}$ & $\begin{array}{l}\text { G-nCM or } \\
\text { nPF } \\
\text { L-nCM } \\
\text { G-AD } \\
\text { L-AD }\end{array}$ & $f(X, \alpha)$ & SDM & Yes & Yes \\
\hline Kuo, 1983 & $\begin{array}{l}\text { FTS, L-H } \\
\text { WGS, L-H }\end{array}$ & $\begin{array}{l}\mathrm{H}_{2}, \mathrm{CO} \\
\mathrm{H}_{2} \mathrm{O}, \mathrm{CO}_{2}\end{array}$ & $\begin{array}{l}\text { G-PF } \\
\text { SL-PF } \\
\text { CMorAD }\end{array}$ & $f(X, \alpha)$ & uniform & $\begin{array}{l}\text { iso- } \\
\text { thermal }\end{array}$ & Yes \\
\hline $\begin{array}{l}\text { Stern, et al., } \\
1985\end{array}$ & $\begin{array}{l}\text { FTS, } 1^{\text {st }} \\
\text { WGS, }^{\text {nd }}\end{array}$ & $\begin{array}{l}\mathrm{H}_{2}, \mathrm{CO} \\
\mathrm{H}_{2} \mathrm{O}, \mathrm{CO}_{2} \\
\mathrm{C}_{\mathrm{n}} \mathrm{H}_{\mathrm{m}}\end{array}$ & $\begin{array}{l}\text { G-AD } \\
\text { SL-AD }\end{array}$ & $\begin{array}{l}\text { overall } \\
\text { gas MB }\end{array}$ & SDM & $\begin{array}{l}\text { iso- } \\
\text { thermal }\end{array}$ & Yes \\
\hline Bukur, 1983 & FTS, $1^{\text {st }}$ & $\mathrm{H}_{2}$ & $\begin{array}{l}\text { G-PF } \\
\text { SL-CM }\end{array}$ & $f(X, \alpha)$ & uniform & $\begin{array}{l}\text { iso- } \\
\text { thermal }\end{array}$ & Yes \\
\hline $\begin{array}{l}\text { Deckwer et al., } \\
1982\end{array}$ & FTS, $1^{\text {st }}$ & $\mathrm{H}_{2}$ & $\begin{array}{l}\text { G-AD } \\
\text { SL-AD }\end{array}$ & $f(X, \alpha)$ & SDM & $\begin{array}{l}\text { iso- } \\
\text { thermal }\end{array}$ & Yes \\
\hline
\end{tabular}

\subsection{Physical Properties of FT Systems}

\subsubsection{Fischer-Tropsch waxes and solvent}

It is known that the hydrodynamics of Fischer-Tropsch Synthesis process is greatly affected by the fluid physical properties of the waxes. Due to the high temperature and high pressure operating conditions for Fischer-Tropsch Synthesis, it is hard to conduct experiments at the matching conditions of Fischer-Tropsch Synthesis. Therefore, other hydrocarbons with similar physical properties as Fischer-Tropsch waxes under the operating conditions have been used to simulate the actual waxes to study the hydrodynamics of Fischer-Tropsch Synthesis. Several studies have been reported in the literature about the physical properties of different Fischer-Tropsch waxes and solvents used for the mimicking purpose (Gormley, et al., 1997; Marano and Holder, 1997; Patel, 
et al., 1990; Soong, et al., 1997). Table 2.2 shows the reported values of Fischer-Tropsch waxes and solvents. It can be observed that the density of reported Fischer-Tropsch waxes range from $645-849 \mathrm{~kg} / \mathrm{m}^{3}$. The viscosity of waxes on the other hand ranges from 0.41 to $71.9 \mathrm{cP}$. In general, the viscosity of most of the waxes used is around 2 to $4 \mathrm{cP}$. The range of surface tension stays reasonably constant between 16 and 28 dyne $/ \mathrm{cm}$.

Other than experimental data, models have been developed to predict the physical properties of Fischer-Tropsch liquids. Tables 2.3 and 2.4 show the asymptotic behavior correlations developed by Marano and Holder (1997) to estimate the properties of $n$ paraffins. The molar volume information derived from the correlation can be converted into density information by dividing the molar volume with the molecular weight of the n-paraffins used. 
Table 2.2. The Physical Properties of Fischer Tropsch Waxes (Gormley, et al., 1997; Marano and Holder, 1997; Patel, et al., 1990; Soong, et al., 1997).

\begin{tabular}{|c|c|c|c|c|}
\hline Fischer Tropsch Wax & $\begin{array}{c}\text { Temperature } \\
\left({ }^{\circ} \mathrm{C}\right)\end{array}$ & $\begin{array}{l}\text { Density } \\
(\mathrm{kg} / \mathrm{m3})\end{array}$ & $\begin{array}{c}\text { Viscosity } \\
\text { (cp) }\end{array}$ & $\begin{array}{c}\text { Surface } \\
\text { Tension } \\
\text { (dyne/cm) }\end{array}$ \\
\hline ACPI wax & 121 & - & 1.7 & - \\
\hline \multirow[t]{5}{*}{ Arge wax } & 121 & - & 5.7 & - \\
\hline & 150 & - & 4.2 & 24 \\
\hline & 200 & - & 2.9 & 20 \\
\hline & 230 & - & 2.5 & 19 \\
\hline & 265 & - & - & 16 \\
\hline \multirow[t]{3}{*}{$\mathrm{C} 28 \mathrm{H} 58$} & 110 & - & 20 & - \\
\hline & 130 & - & 19.8 & - \\
\hline & 150 & - & 5.6 & - \\
\hline \multirow{3}{*}{ d. Allied-AC-1702 } & 110 & - & - & - \\
\hline & 130 & - & 71.9 & - \\
\hline & 150 & - & 46.3 & - \\
\hline \multirow[t]{5}{*}{ Drakeol-10 Oil } & 20 & 849 & 38.13 & 28.3 \\
\hline & 100 & 806 & 3.38 & 23.9 \\
\hline & 175 & - & - & 19.9 \\
\hline & 200 & 743.9 & 0.892 & - \\
\hline & 265 & 698 & 0.55 & - \\
\hline \multirow[t]{6}{*}{ FT-200 Wax } & 150 & - & 4.4 & - \\
\hline & 200 & - & 2.8 & - \\
\hline & 230 & - & 2.4 & - \\
\hline & 149 & - & 4.4 & - \\
\hline & 204 & - & 2.2 & - \\
\hline & 260 & - & 1.7 & 24 \\
\hline \multirow[t]{4}{*}{ FT-300 Wax } & 150 & - & 6.4 & 24 \\
\hline & 200 & 722 & 4.2 & 21 \\
\hline & 230 & - & - & 19 \\
\hline & 265 & 681 & 2.7 & 17 \\
\hline F-T 1t. Cut & 25 & - & - & 23 \\
\hline \multirow{3}{*}{ F-T med. Cut } & 25 & - & 2.36 & 26 \\
\hline & 100 & - & 0.842 & - \\
\hline & 150 & - & 0.555 & - \\
\hline Kogasine & 145 & - & 0.41 & 18 \\
\hline \multirow[t]{3}{*}{ Krupp wax } & 200 & - & 3.0 & - \\
\hline & 230 & - & 2.2 & - \\
\hline & 260 & - & 1.6 & - \\
\hline \multirow[t]{3}{*}{ Mobil run 3} & 149 & - & 2.8 & \\
\hline & 204 & - & 1.7 & - \\
\hline & 260 & - & - & $26-27$ \\
\hline
\end{tabular}




\begin{tabular}{|c|c|c|c|c|}
\hline Mobil run 4 & $\begin{array}{l}149 \\
204 \\
260 \\
\end{array}$ & $\begin{array}{l}- \\
- \\
-\end{array}$ & $\begin{array}{l}6.1 \\
4.3 \\
3.4 \\
\end{array}$ & $\begin{array}{c}- \\
- \\
26-27\end{array}$ \\
\hline Mobil run 5 & $\begin{array}{l}149 \\
204 \\
260\end{array}$ & $\begin{array}{l}- \\
-\end{array}$ & $\begin{array}{c}17.6 \\
8.5 \\
5.5\end{array}$ & $\begin{array}{c}- \\
- \\
28\end{array}$ \\
\hline Mobil run 7 & $\begin{array}{l}149 \\
204 \\
260\end{array}$ & $\begin{array}{l}- \\
-\end{array}$ & $\begin{array}{l}8.2 \\
4.1 \\
2.3\end{array}$ & $\begin{array}{c}- \\
- \\
26-27\end{array}$ \\
\hline Mobil comp & $\begin{array}{l}150 \\
200 \\
230\end{array}$ & $\begin{array}{l}- \\
- \\
-\end{array}$ & $\begin{array}{l}6.5 \\
3.8 \\
3.1\end{array}$ & $\begin{array}{l} \\
- \\
-\end{array}$ \\
\hline Mobil Wax & $\begin{array}{l}121 \\
200 \\
265 \\
\end{array}$ & $\begin{array}{c}- \\
716 \\
674 \\
\end{array}$ & $\begin{array}{l}9.5 \\
3.8 \\
2.3 \\
\end{array}$ & $\begin{array}{l}- \\
-\end{array}$ \\
\hline Mobil FT Wax & $\begin{array}{l}110 \\
130 \\
150\end{array}$ & $\begin{array}{l}- \\
- \\
-\end{array}$ & $\begin{array}{l}26.6 \\
19.4 \\
17.2\end{array}$ & $\begin{array}{l}- \\
- \\
-\end{array}$ \\
\hline P \& W wax & $\begin{array}{l}100 \\
150\end{array}$ & - & $\begin{array}{l}1.829 \\
0.995\end{array}$ & - \\
\hline Paraflint & 121 & - & 9.5 & - \\
\hline Paraffin wax & $\begin{array}{l}145 \\
175 \\
200 \\
220 \\
250 \\
260 \\
275 \\
300\end{array}$ & $\begin{array}{l}- \\
- \\
- \\
- \\
- \\
- \\
- \\
-\end{array}$ & $\begin{array}{c}13.0 \\
8.1 \\
5.7 \\
4.0 \\
2.8 \\
2.0 \\
1.8 \\
1.2\end{array}$ & $\begin{array}{l}29.1 \\
27.0 \\
25.2 \\
24.0 \\
21.8 \\
21.2 \\
20.4 \\
19.8\end{array}$ \\
\hline Polywax-655 & $\begin{array}{l}110 \\
130 \\
150 \\
\end{array}$ & $\begin{array}{l}- \\
- \\
-\end{array}$ & $\begin{array}{l}20.6 \\
14.5 \\
10.6 \\
\end{array}$ & $\begin{array}{l} \\
- \\
-\end{array}$ \\
\hline Sasol Wax & $\begin{array}{l}200 \\
265 \\
\end{array}$ & $\begin{array}{l}701 \\
655 \\
\end{array}$ & $\begin{array}{l}2.9 \\
2.0 \\
\end{array}$ & - \\
\hline UCC wax & 121 & - & 2.8 & - \\
\hline n-Paraffins & $0-300^{\circ} \mathrm{C}$ & \multicolumn{3}{|c|}{ See Table 2} \\
\hline
\end{tabular}


Table 2.3. Temperature-Independent Parameters for Properties of $n$-Paraffins

$Y=Y_{\infty, 0}+\Delta Y_{\infty}\left(n-n_{0}\right)-\Delta Y_{0} \exp \left(-\beta\left(n \pm n_{0}\right)^{\gamma}\right)$

$\left(n+n_{0}\right)$ for molar volume, $\left(n-n_{0}\right)$ for all others

\begin{tabular}{|l|c|c|c|}
\hline & $\begin{array}{c}\text { Molar volume } \\
(\mathrm{cm} 3 / \mathrm{gmol})\end{array}$ & ln viscosity $(\mathrm{cP})$ & Surface tension \\
\hline$n_{0}$ & -1.388524 & -2.293981 & 0.264870 \\
\hline$\Delta Y_{0}$ & $\mathrm{a}$ & $\mathrm{a}$ & $\mathrm{a}$ \\
\hline$Y_{\infty, 0}$ & 0 & 57.8516 & $\mathrm{a}$ \\
\hline$\Delta Y_{\infty}$ & $\mathrm{a}$ & $\mathrm{a}$ & 0 \\
\hline$\beta$ & 0.183717 & 2.476409 & 2.511846 \\
\hline$\gamma$ & 0.753795 & 0.0112117 & 0.201325 \\
\hline
\end{tabular}

${ }^{\mathrm{a}}$ See Table 3

Table 2.4. Temperature-Dependent Parameters for Properties of $n$-Paraffins

$\Delta Y=A+B / T+C \ln T+D T^{2}+E / T^{2}$ for $\ln \mu_{\mathrm{L}}$

$\Delta Y$ or $Y=A+B T+C T^{2}+D T^{3}$ for $V_{L}, \sigma$

\begin{tabular}{|l|c|c|c|c|c|c|}
\hline & \multicolumn{2}{|c|}{ Molar volume (cm3/gmol) } & \multicolumn{2}{c|}{ ln viscosity $(\mathrm{cP})$} & \multicolumn{2}{c|}{ Surface tension $(\mathrm{dyne} / \mathrm{cm})$} \\
\hline & $\Delta Y_{0}$ & $\Delta Y_{\infty}$ & $\Delta Y_{0}$ & $\Delta Y_{\infty}$ & $\Delta Y_{0}$ & $\Delta Y_{\infty}$ \\
\hline$\underline{\mathbf{A}}$ & 8592.30 & 12.7924 & -602.688 & 0.0290196 & 627.213 & 73.8715 \\
\hline$B$ & -85.7292 & 0.0150627 & 77866.8 & -241.023 & -0.882888 & -0.177123 \\
\hline$C$ & 0.280284 & $-130794 \times 10^{-}$ & 198.006 & 0.0440959 & 0.00268188 & $1.54517 \times 10^{-}$ \\
& & 5 & & & 0 & 0 \\
\hline$D$ & $\begin{array}{c}-4.48451 \times 10^{-} \\
4\end{array}$ & $1.59611 \times 10^{-4}$ & $\begin{array}{c}-4.18077 \times 10^{-} \\
7\end{array}$ & $\begin{array}{c}-1.84891 \times 10^{-} \\
7\end{array}$ & 0 & \\
\hline$E$ & & - & 56561.7 & & $0-150^{\circ} \mathrm{C}$ \\
\hline$\Delta T$ & $0-300^{\circ} \mathrm{C}$ & $0-300^{\circ} \mathrm{C}$ & $0-300^{\circ} \mathrm{C}$ & $0-300^{\circ} \mathrm{C}$ & $0-150^{\circ} \mathrm{C}$ & 0 \\
\hline
\end{tabular}

\subsubsection{Solids Phase (Catalyst)}

Sabatier and Sanderson found in 1902 that methane can be obtained when hydrogen and carbon monoxide react over nickel or cobalt catalyst (Storch et al., 1951). Fischer and Tropsch in 1923 obtained a high yield of liquid products by using iron based alkali promoted catalyst. Process was later studied using iron, cobalt and nickel catalysts. First industrial scale and all pre and during WWII processes in Germany used cobalt based catalyst, 100Co:5ThO:8MgO:200Kieselguhr, because of its high activity. Due to the shortage in supply of Cobalt during WWII new iron based catalysts were developed. Ruhrchemie company developed a series of iron based catalysts, 100Fe:xCu:yK:zSiO2. South African commercial, ARGE, process still uses this type of a catalyst ( $x: y: z=4.3: 4.1: 25)$. Iron although less active is much cheaper and more easily available than cobalt. Srivastava et al. (1990) and Rao et al. (1992) reviewed various iron based FT catalysts. Nickel has been the most often used in combination with some other active component, such as cobalt. Ruthenium is the forth originally proposed Fisher-Tropsch 
(FT) catalyst. However, ruthenium has been similarly as nickel mostly used in combination catalysts because it is expensive (two orders of magnitude more expensive than cobalt) and has much higher range of optimum working pressures compared to iron or cobalt. Until today thousands of different catalyst formulations have been tested and hundreds of active catalysts have been proposed with iron and cobalt based catalysts being the most common commercially. Recently more studies have been directed toward the cobalt based FT catalysts because of their high activity and modular selectivity. Iglesia (1997) and Oukachi et al. (1999) reviewed various cobalt based FT catalysts. Since catalyst activity and selectivity (which are affected by catalyst porosity, tortuosity, density which is usually greater than $5 \mathrm{gm} / \mathrm{cm}^{3}$, surface composition and structure, wetability, etc.) are determined by catalyst chemical composition, type of carrier (catalyst support), catalyst preparation process, activation procedure, etc. The FT catalyst properties vary noticeably.

Traditionally the most often used support (binder) of FT catalysts have been silica $\left(\mathrm{SiO}_{2}\right)$ and alumina $\left(\mathrm{Al}_{2} \mathrm{O}_{3}\right)$ (density between 1.5 to $3 \mathrm{gm} / \mathrm{cm}^{3}$ ). Some early German catalysts used the Kieselguhr. In addition to these supports, titania $\left(\mathrm{TiO}_{2}\right)$, magnesia $(\mathrm{MgO})$, active carbon and zeolites (e.g. ZSM-5) have been somewhat less frequently used (Storch et al., 1951).

Hence, hydrodynamics obtained using one catalyst may not represent a general information for all the catalyst types. Using only FT catalyst supports for hydrodynamics investigation does not either provide representative information due to the difference in the particle physical and mechanical properties. Therefore, in the open literature, most of the investigations on the hydrodynamics of cold-flow model slurry bubble column reactors have been performed using glass beads of different sizes and airwater/hydrocarbon system (Ong et al., 2000). 
The experimental facilities and the diagnostic techniques needed for the project have been prepared, developed, and tested for the investigations performed at Washington University (WU), and Ohio State University (OSU) as indicated below. CARPT/CT techniques have been advanced to be used in high pressure slurry bubble column reactors

\subsection{High pressure and high temperature 2" diameter slurry bubble column [OSU]}

The schematic diagram of the high pressure and high temperature slurry bubble column is shown in Figure 3.1, which was arranged for the tasks set for this project. The height of the column is $95.9 \mathrm{~cm}$ and has an inside diameter of $5.1 \mathrm{~cm}$. There are three pairs of quartz windows installed on the front and rear sides of the column. These windows allow viewing throughout the entire test section of the column. Each window is of $1.27 \mathrm{~cm}$ in width and $9.3 \mathrm{~cm}$ in height. The maximum operating pressure and temperature of the system are $21 \mathrm{MPa}$ and $180^{\circ} \mathrm{C}$, respectively. Additionally, a perforated plate is used as the gas distributor comprised with 19 triangular pitched holes of $0.45 \mathrm{~mm}$ diameter each and \% open area is 0.156 . A dynamic pressure transducer is installed at $1.0 \mathrm{~cm}$ and 20.5 $\mathrm{cm}$ above the distributor plate.

\subsection{High pressure 6" diameter slurry bubble column [WU]}

The experimental setup shown in Figure 3.2 was designed to support the maximum operating pressure of $200 \mathrm{psig}$. The unit designated for CT/CARPT techniques was prepared to carry the experiments needed for the project while the second unit which is equipped with windows and ports was developed as a part of this project and other funded projects that are executed in parallel (Industrial Consortium and DF-2295PC95051). The air is supplied from two compressors connected in parallel with the working pressure of $195 \mathrm{psig}(1.45 \mathrm{MPa})$ and the maximum corresponding rated flow rate of 310 SCFM. The compressed atmospheric air is purified, by passing through the dryer and several air filter units. The maximum operational flow rate through the column is about 230 SCFM at atmospheric pressure and about 130 SCFM at 1.0 MPa. The air flow rate is regulated using a pressure regulator and rotameter setup consisting of 4 rotameters of increasing range connected in parallel. Air exits the column through a demister, passes through the backpressure regulator (that controls column operating pressure) and vents to atmosphere. A laboratory scale $16.15 \mathrm{~cm}(6$ ") diameter stainless steel bubble column is used in all experiments. Column design enables easy removal of the distributor chamber and sparger replacement. Two similar column designs are used to suit all the needed experiments. The first one, designed for CARPT/CT experiments, is a 6" column equipped with just two probe ports (1") at each end of the column (i.e. $z=215 \mathrm{~cm}$ and $\mathrm{z}$ $=12 \mathrm{~cm}$ ) as shown in Figure 3.3. The second column is used for overall gas holdup and differential pressure (DP) measurements, which has the same dimensions as the first one (Figure 3.4). This column is equipped with an array of additional 15 probe ports (1") and $6\left(12\right.$ "H x $1 \frac{1}{2}$ "W) view windows. The three view windows mounted at radially opposite sides are staggered to cover the middle and the top part of the column. View windows are made of tempered quartz glass and are rated to the same pressure as the column itself 
(150 psig). These windows and 1" ports are mounted on two mutually perpendicular r-z planes. The batch of slurry constitutes the selected solvent as the liquid phase and the selected solid phase.

\subsection{Dynamic Gas Disengagement (DGD)[OSU]}

Gas holdup measurements were made using dynamic gas disengagement (DGD) technique. The procedure in the DGD technique includes: 1) the gas supply to the column is suddenly shut off; 2) the gas holdup is continuously measured, e.g., based on the bed height. A typical DGD curve (pressure drop, (dp/dz) vs. time) for the high pressure slurry bubble column is shown in Figure 3.5. The gas flow is shut off at $t=9$ second. The entire process can be divided into 6 stages: (1) A sudden increase in the differential pressure signal is observed immediately after the gas shut-off, which corresponds to simultaneous escape of bubbles of various sizes. (2) The increase in the signal is much more gradual due to the faster disengagement of larger bubbles. (3) The differential pressure remains at a relatively constant value for the next 150 seconds approximately as the particles are still fully suspended by the liquid motion induced by the bubbles. (4) At $t=200$ second, the signal starts to increase gradually as the particles start settling down, which leads to an increased solid concentration in the region between the two pressure ports. The solids surface starts to move downwards. (5) The solid surface continues moving down and increasing amount of particles completely settle down on the bottom of the column, which causes the sudden drop in the differential pressure signal at $550<t<800$ second. (6) All the particles settle down at $\mathrm{t}>800$ second. The pressure drop signal can be related to the gas holdup $\left(\varepsilon_{g}\right)$ by

$$
\varepsilon_{g}=\frac{\left[(\Delta P / \Delta z)_{d}-(\Delta P / \Delta z)_{d}^{0}\right] / g}{\left(\rho_{g}-\rho_{l}\right)-\left[(\Delta P / \Delta z)_{d}^{0}\right] / g}
$$

where $(\Delta P / \Delta z)_{d}$ is the dynamic pressure gradient as the gas shut-off and $(\Delta P / \Delta z)_{d}^{0}$ is the dynamic pressure gradient at stage (3) mentioned above, i.e., in a gas-free slurry suspension. $\rho_{g}$ and $\rho_{l}$ are the densities of gas and liquid, respectively. In deriving Eq. (3.1), it is assumed that the ratio of the solids holdup to the liquid holdup in stage 3 is the same as that in the steady-state slurry bubble column. Thus, the gas holdup in the high pressure slurry bubble column can be calculated from Equation (3.1).

\subsection{Fiber Optic Probe [OSU]}

The direct measurements of bubble sizes and bubble rise velocity in the high-pressure slurry bubble column were conducted using an U-shaped fiber optic probe which was prepared to be used in 2" high pressure slurry bubble column. The probe utilizes the difference in refractive index of gas, liquid, and solids to distinguish the gas phase from the liquid-solid suspension. Schematic diagram of the optical probe is shown in Figure 3.6. The fiber cladding in the tip portion is partially removed in such a manner that it yields the most distinctive signals for gas void detection. The cross section of the tip is 
perpendicular to the flow direction. The probe has a dimension of $1.2 \times 4 \mathrm{~mm}$. The output of the photo-multiplier is interfaced with a computer data acquisition system, which samples the signal for four seconds at a frequency of $2,000 \mathrm{~Hz}$. The tip of the probe is located at the center of the column and $0.12 \mathrm{~m}$ above the distributor. The probe is calibrated in a chain of bubbles; bubbles passing through the tip periodically. The bubble rise velocity, $u_{b}$ can be calculated by

$u_{b}=\frac{\Delta h}{\Delta \tau_{2}}$

where, $\Delta h$ is the vertical distance between the two tips and $\Delta \tau_{2}$ is the time lag between the rear surface of the bubbles intercepting the upper and lower tips. The result is calibrated against the bubble rise velocity measured with a video camera. It is noted that $\Delta \tau_{2}$ is consistently less than the other time lag $\Delta \tau_{1}$, corresponding to the frontal surface, due to the deformation of the frontal surface upon the interception. The comparison between the two bubble velocities reveals that $\Delta \tau_{2}$ should be used instead of $\Delta \tau_{1}$. The average error for the bubble rise velocity by the probe is less than $5 \%$. The bubble chord length, $l$, is evaluated as

$l=u_{b} \tau$,

where, $\tau$ is the time period when the bubble is in contact with the lower tip of the probe. The probe actually measures the vertical chord length of the bubble rather than the bubble diameter.

\subsection{Laser Doppler Anemometer (LDA) [OSU]}

The Laser Doppler Anemometer is capable of non-intrusive velocity measurements in fluid dynamics in both gas and liquid phases. It was arranged to be used in slurry bubble column for the investigations needed as a part of this project. It has up to three velocity components with high accuracy and high spatial resolution due to small measurement volume. The basic components of an LDA include a continuous wave laser, a traverse system, transmitting and receiving optics and a signal conditioner and a signal processor. Figure 3.7 shows the set-up of LDA system used in this work at OSU. A 300-mW aircooled argon-ion laser and a beam separator are used to generate two pairs of beams of known wavelengths of 514.5 and $480 \mathrm{~nm}$. The light is transmitted through a fiber optic cable and a probe with 3.40 and $3.22 \mu \mathrm{m}$ and measurement volumes of $0.164 \mathrm{X} 0.164 \mathrm{X}$ $2.162 \mathrm{~mm}$ and $0.156 \mathrm{X} 0.156 \mathrm{X} 2.05 \mathrm{~mm}$ for the 514.5 and $480-\mathrm{nm}$ wavelengths respectively. The scattered light is collected with a detector and processed with a signal processor.

The principle of the operation follows that when a particle passes through the intersection volume formed by the two coherent laser beams, the scattered light received by a detector has components from both beams. The components interfere on the surface of the detector. Due to changes in difference between optical path lengths of the two components this interference produces pulsating light intensity as the particle moves through the measurement volume. 


\subsection{Computer Automated Radioactive Particle Tracking (CARPT) [WU]}

Computer Automated Radioactive Particle Tracking (CARPT) technique was first used by Kondukov et al. (1964) to study the particle motion in a fluidized bed. This technique has been used extensively at Washington University (Chemical Reaction Engineering Laboratory) to measure in a non-invasive manner the flow pattern and turbulent parameters of different multiphase flow reactors. CARPT experiment comprises two steps: CARPT calibration ('static' experiment) and the actual CARPT experiment ('dynamic' experiment). The dynamic experiment involves tracking of a single radioactive tracer particle by detecting the intensity distribution of emitted $\gamma$-rays (Figure 3.8). The $\gamma$-ray intensity distribution is detected using an array of $\mathrm{NaI}$ scintillating detectors strategically placed around the studied region of the column. The intensity of gamma ray arriving at each detector decreases with the increasing distance between the detector. The photon count rate obtained at each detector is related to the distance between the source and the detector using 'static' experiment. The instantaneous position of the tracer is then accurately calculated from the distances using an optimized regression scheme. The time differentiation of the displacement yields local velocities. The ensemble averaged velocity profiles and 'turbulent' parameters can then be computed with the aid of algorithms developed at CREL. Due to various advantages, Scandium 46 with the activity of about $200-500 \mu \mathrm{Ci}$ is selected as a radioactive particle. In this work, the objective is to compute solids instantaneous velocities, radial profile of axial solids velocity and 'turbulent' parameters, therefore a radioactive particle of the same size and density of the solids is essential to monitor the motion of solids in slurry bubble column reactors. Scandium is a highly reactive rare earth metal whose reactivity increases with decrease in diameter of the particle. To resolve the issue of the reacting scandium tracer particle we have developed a new technique for coating and protecting the minute size tracking particles. A tracer scandium Sc46 particle of required diameter is protected with a thin coating of Parylene $\mathrm{N}$, an extremely inert derivative of poly p-xylene with excellent thermal and mechanical properties. The coated Scandium particle is then irradiated in a nuclear reactor. The resulting radioactive scandium Sc46 particle (strength of up to $200 \mu \mathrm{Ci}$ and half-life of 83 days) with a total diameter within the solid phase particle size range is thus used as a tracer particle. Since the density of Parylene $\mathrm{N}$ is $1.11 \mathrm{~g} / \mathrm{cm}^{3}$, application of different coating thickness lower the overall particle density from $2.99 \mathrm{~g} / \mathrm{cm}^{3}$ (of pure scandium) to about $2 \mathrm{~g} / \mathrm{cm}^{3}$.

A detailed experimental setup and calculation procedure for CARPT experiments is given in Degaleesan (1997) and Rados (2003). In-situ calibration of detectors has been performed under the desired operating conditions using a calibration device that is operated under high pressure. CARPT data (tracer particle position in time) acquired over sufficiently long time, to ensure enough particle occurrences in each column cell and good time/ensemble averaging, is used for calculation of the time averaged solids
a) velocities,
b) "Reynolds" stresses,
c) "turbulent" kinetic energy and
d) eddy diffusivities. 
This unique technique is essential for validation of hydrodynamic models used in design and scale-up and to test the effect of different design and operation variables (e.g. pressure, gas velocity, distributor design, internals, etc.) on the flow patterns in FT slurry bubble column reactors.

Challenging technical problems were encountered during the implementation of CARPT in high pressure stainless steel column, which were all resolved successfully. These problems are outlined and discussed in Appendix F.

\subsection{Computed Tomography (CT) [WU]}

Computed Tomography (CT) is used for measurement of the cross-sectional phase holdup distribution in multiphase systems (Figure 3.11). CT technique has been extensively implemented at Washington University on various multiphase flow systems. It consists of an array of detectors with an opposing source, which rotate together around the object to be scanned. The scanner uses a Cesium (Cs-137) encapsulated $\gamma$-ray source with activity of $85 \mathrm{mCi}$. The array of detectors and the source are mounted on a gantry, which can be rotated about the object to be scanned through a step motor. The entire system is completely automated to acquire the data needed for the reconstruction of the phase distribution in a given cross-section. After detail analysis of various algorithms, Kumar 1994) implemented Estimation-Maximization Algorithm (EM Algorithm) for image reconstruction. It is based on maximum likelihood principles and takes into account the stochastic nature of the projection measurements.

Single source CT is used for phase holdup reconstruction in two-phase (e.g., gas-liquid) systems. Theoretically, dual source CT is capable of resolving the holdups in three phase systems (e.g., gas-liquid-solid). In this work in the absence of dual energy/source CT technique, two methodologies have been proposed viz; CARPT/CT/DP and CT/DP, to calculate holdup profiles of all three phases in a slurry system using single $\gamma$-ray source (Rados, 2003).

For a single $\gamma$ radiation source, absorbance $A$ over the path $l$ is equal to:

$$
A=-\ln \frac{I}{I_{0}}=\sum_{l}(\rho \mu)_{i j} l_{i j}
$$

where $I_{0}$ is the intensity of radiation emitted by the source, $I$ is the intensity of radiation received by the detector. $\Sigma$ indicates the summation of the volumetric attenuation $(\rho \mu)_{i j}$ of each cell $i j$ multiplied by the path length in that cell $l_{i j}$ along the path $l$, through which the radiation beam passes on its way from the source to the detector. If sufficiently large number of the scans of the operating column are taken from different directions when the volumetric attenuation in each cell $(\rho \mu)_{i j}$ can be calculated. To get the holdup distribution we have to measure the absorbance $A_{K}$ for an empty column $(K=G)$, for a column filled with liquid $(K=L)$, for a column with solids and gas in voids between solid particles $(K=G S)$ and for a column in operation with gas-liquid-solid slurry $(K=G L S)$. For each of these situations the detected intensity of radiation $I_{K}$ and hence the measured absorbance $A_{K}$ is different. Since the flow is time dependent larger number of acquired projections 
than cells (\#equations >> \#unknowns, over sampling) will yield more accurate time averaged attenuation coefficients (better statistics). In general $I_{0}$ is unknown and because of that the intensity of radiation $I_{K}$ must be normalized with the intensity of radiation detected in the column containing only the gas phase $I_{G}$. In addition the intensity $I_{K}$ must be corrected for the background (room) radiation intensity $I_{K, b c k}$. This yields the following equation for $A_{K}$ :

$$
A_{K}=-\ln \frac{I_{K}-I_{K, b c k}}{I_{G}-I_{G, b c k}}=\sum_{l}\left[(\rho \mu)_{K, i j}-(\rho \mu)_{G, i j}\right] l_{i j}
$$

One defines relative volumetric attenuation as:

$$
R_{K, i j}=(\rho \mu)_{K, i j}-(\rho \mu)_{G, i j}
$$

For the column containing packed bed of solids (uniform holdup of $\varepsilon_{S}^{0}$ ) and gas in voids between the solids particles the volumetric attenuation coefficient in cell $i j$ is equal to:

$$
(\rho \mu)_{G S, i j}=(\rho \mu)_{S, i j} \varepsilon_{S}^{0}+(\rho \mu)_{G, i j}\left(1-\varepsilon_{S}^{0}\right)
$$

Substitution of eq. (3.5b) into eq. (3.6) (written for the gas-solid system, $K=G S$ ) after some manipulation yields the local solids volumetric attenuation coefficient:

$$
(\rho \mu)_{S, i j}=\frac{R_{G S, i j}+(\rho \mu)_{G, i j} \varepsilon_{S}^{0}}{\varepsilon_{S}^{0}}
$$

Similarly for a slurry system,

$$
(\rho \mu)_{G L S, i j}=(\rho \mu)_{G, i j} \varepsilon_{G, i j}+(\rho \mu)_{L, i j}\left(1-\varepsilon_{G, i j}-\varepsilon_{S, i j}\right)+(\rho \mu)_{S, i j} \varepsilon_{S, i j}
$$

Eq. (3.8) combined with eq. (3.5b) (written for liquid, $K=L$ and slurry, $K=G L S$ ) and eq. (3.7) yields the expression for local gas holdup (cell $i j$ ):

$$
\varepsilon_{G, i j}=\frac{R_{G S, i j} \frac{\varepsilon_{S, i j}}{\varepsilon_{S}^{0}}+R_{L, i j}\left(1-\varepsilon_{S, i j}\right)-R_{G L S, i j}}{R_{L, i j}}
$$

In order to solve the above system of equations for construction of the three phases distribution we need one more equation for local solids holdup, $\varepsilon_{S, i j}$. In dual source CT one more equation of the form (3.9) can be written for the other $\gamma$ source or energy. However to evaluate three phase holdups with the current state of single source CT facility, two methods with some assumptions have been proposed during this work. These methods, essentially, generate additional equation needed to solve the above system of equations. These methods are as follows,

a) $C A R P T / C T / D P$ where the needed equation has been obtained from CARPT, DP, and overall gas holdup measurements.

b) CT/Overall Gas holdup where the needed equation has been obtained from DP equation along with overall gas holdup measurement. 
The above two methodologies are outlined in the following paragraphs. Based on the obtained results and findings method (b) is preferred and hence it has been used for the data processing. The shortcomings associated with these methods are discussed as well.

\section{CARPT/CT/DP method}

Here, the additional equation is generated from differential pressure (DP), CARPT, and overall gas holdup measurements as follows

$$
-\frac{1}{g} \frac{\Delta P}{\Delta z}=\rho_{G} \overline{\varepsilon_{G}}+\rho_{L}\left(1-\overline{\varepsilon_{G}}-\overline{\varepsilon_{S}}\right)+\rho_{S} \overline{\varepsilon_{S}} \quad \text { DP }
$$

DP Equation (3.10) assumes fully developed flow, no axial holdup profiles and negligible wall shear stress in the section $\Delta z$. Fully developed flow in bubble columns is usually reached at heights above two column diameters. Axial holdup profiles can be neglected over small $\Delta z$ distances and the wall shear stress has been shown to be negligible compared to the pressure drop (Fan, 1989).

$$
\varepsilon_{S, i j}=n_{S, i j} \frac{\overline{\varepsilon_{S}}}{\overline{n_{S}}}
$$

Equation (3.11) states that the volume averaged number of radioactive tracer particle occurrences in the specific cell $n_{S, i j}$ is proportional to the solids holdup in that cell assuming that the radioactive tracer particle completely resembles solids phase particles and that all the cells in the considered cross plane are well perfused and readily accessible to the radioactive tracer particle (Moslemian et al., 1992). This assumption may not be justified and it is questionable.

Combining equation (3.10) and (3.11) yields the following expression for the local solids holdup (cell $i j)$

$$
\varepsilon_{S, i j}=\frac{-\frac{1}{g} \frac{\Delta P}{\Delta z}-\rho_{G} \overline{\varepsilon_{G}}-\rho_{L}\left(1-\overline{\varepsilon_{G}}\right)}{\rho_{S}-\rho_{L}} \times \frac{n_{S, i j}}{\overline{n_{S}}}
$$

Using the following iterative procedure the holdup profiles of all three phases can be calculated.

1) Guess the cross-sectional average solids holdup. The initial guess is based on the calculation of the cross-sectional average solids holdup from the overall gas holdup measurements and nominal solids loading $\left(\mathrm{v}_{S 0}\right.$, volume of solids per volume of slurry suspension initially charged into the column) using the equation $\overline{\varepsilon_{S}}=\mathrm{v}_{S 0}\left(1-\overline{\varepsilon_{G}}\right)$.

2) Using Equation (3.11) calculate the solids holdup in each cell.

3) Using Equation (3.9) calculate the gas holdup in each cell.

4) Calculate the cross-sectional average gas holdup.

5) Using Equation (3.12) calculate the solids holdup in each cell.

6) Calculate the cross-sectional average solids holdup. 
7) Compare the calculated and previous values (initial guess in the first iteration) of the cross-sectional average solids holdup.

Using the solids holdups in each cell recalculated in step 5) repeat the steps 3) through 7) until convergence with specified tolerance is achieved.

The results and findings of CARPT/CT/DP method that has been originally proposed (DOE Reports $1^{\text {st }}$ and $2^{\text {nd }}$ Year) to obtain three-phase holdup profiles by combining CARPT, CT, and DP measurements, have been carefully evaluated which lead to the following conclusions:

a) The holdup profiles obtained using the $\mathrm{CT} / \mathrm{DP} / \mathrm{CARPT}$ procedure are too sensitive to the measurement of the pressure drop $\Delta \mathrm{P}$.

Equation (3.10) can be rearranged to obtain general relationship between crosssectional gas and solids holdup as follows

$$
\overline{\varepsilon_{S}}=A+B \overline{\varepsilon_{G}}
$$

where,

$$
\begin{aligned}
& \mathrm{A}=\frac{-\frac{\mathrm{E}}{\mathrm{E}_{\mathrm{C}}} \frac{\Delta \mathrm{z}_{\mathrm{C}}}{\Delta \mathrm{z}}}{\rho_{\mathrm{S}} / \rho_{\mathrm{L}}-1}=-0.132 \mathrm{E} \\
& B=\frac{1}{\rho_{S} / \rho_{L}-1}
\end{aligned}
$$

The obtained CT data using air-water-glass beads system has been processed by CARPT/CT/DP methodology. It has been found that, pressure drop must be measured within $0.4 \mathrm{~mm} \mathrm{H}_{2} \mathrm{O}$ accuracy. The small variation in the measured $\mathrm{E}$ (volt) value affect significantly the reconstructed three phases holdups distribution. The ratted accuracy of the used differential pressure transducer (DP) setup is \pm 1.4 $\mathrm{mmH}_{2} \mathrm{O}(\Delta \mathrm{A}= \pm 0.004, \Delta \mathrm{E}= \pm 0.028 \mathrm{~V})$ while the signal fluctuations during the gas free data acquisition (zero and span calibration) could be as high as $\pm 5.0 \mathrm{mmH}_{2} \mathrm{O}$ $(\Delta \mathrm{A}= \pm 0.013, \Delta \mathrm{E}= \pm 0.100 \mathrm{~V})$. This means that the present DP setup does not have the needed accuracy to be reliably used in the sensitive CT/DP/CARPT procedure.

b) The radioactive particle occurrences from CARPT measurements are utilized as follows to calculate the radial solids holdup profile trend. This relationship is questionable and it has not been validated.

$$
\frac{\varepsilon_{S(r)}}{\overline{\varepsilon_{S}}}=\frac{n_{S(r)}}{\overline{n_{S}}}
$$


Hence, the solids loading calculated using the CT/DP/CARPT methodology has been found to be higher in the center of the column than at the wall. This implies that the gas concentrates the solids in the center of the column, which is physically unrealizable. The actual solids loading profiles are found to be either flat (uniformly concentrated slurry) as was reported by several authors (Hu et al., 1986; Badgujar et al., 1986) or higher at the wall since the gas pushes aside the heavier solid particles.

Due to the above mentioned reasons, CT/Overall gas holdup methodology have been proposed as explained below. This methodology combines CT and overall gas holdup measurements along with pressure drop (DP) working equation. It has been used to process the obtained $\mathrm{CT}$ data.

\section{$\underline{\text { CT/Overall Gas holdup method }}$}

This methodology is based on following two assumptions,

a) axially invariant gas holdup, $\partial \overline{\varepsilon_{\mathrm{G}}} / \partial \mathrm{z}=0$

b) uniform cross-sectional solids loading, $\left(\frac{v_{s}}{v_{s}+v_{L}}\right)$

Both of these two assumptions seem to be quite reasonable at certain operating conditions and are supported by many previous studies (Matsumoto et al., 1992; Bukur et al., 1996; Badgujar et al., 1986; Limtrakul, 1996). However, the shortcoming of this method is that the above stated assumptions would not be valid at all operating conditions and at all the column heights.

This method utilizes generalized DP working equation as follows,

$\overline{\varepsilon_{\mathrm{S}}}=\mathrm{A}+\mathrm{B} \overline{\varepsilon_{\mathrm{G}}}$

where, A in this method is considered as a fitting parameter (rather than a measured value) that represents the pressure drop corresponding to the above assumption made at the used operating conditions, while $\mathrm{B}$ is a function of solids and liquid density (Equation 3.14b).

The solids loading across the cross-section is defined as,

$$
\overline{v_{S}}=\frac{\overline{\varepsilon_{S}}}{1-\overline{\varepsilon_{G}}}
$$

Due to uniform solids loading along the cross-section, we can write Equation (3.14) as

$$
\varepsilon_{S, i j}=\overline{v_{S}}\left(1-\varepsilon_{G, i j}\right)
$$


Combining equations (3.9), (3.13), (3.15), (3.16), yields the following working CT equation,

$$
\varepsilon_{G, i j}=1-\frac{R_{G L S, i j}}{\overline{\frac{v_{S}}{\varepsilon_{S}^{0}}} R_{G S, i j}+\left(1-\overline{v_{S}}\right) R_{L, i j}}
$$

The iterative procedure used to compute gas and solids holdup profile using this methodology is as follows,

1. Guess value of A.

2. With assumed value of gas holdup (assumption 1 i.e. cross-sectional gas holdup $=$ overall gas holdup), calculate $\overline{\varepsilon_{G}}$, (Equation 3.13).

3. Calculate the gas holdup cross-sectional profiles $\varepsilon_{\mathrm{G}, \mathrm{ij}}$, Equation $(3.15,3.17)$.

4. Calculate the solids holdup cross-sectional profiles $\varepsilon_{\mathrm{S}, \mathrm{ij}}$, Equation $(3.15,3.16)$.

5. Calculate gas and solids radial profiles, $\varepsilon_{\mathrm{G}}(\mathrm{r}), \varepsilon_{\mathrm{S}}(\mathrm{r})$ (azimuthal averaging).

6. Calculate the cross-sectional average gas and solids holdups, $\overline{\varepsilon_{\mathrm{G}}}, \overline{\varepsilon_{\mathrm{S}}}$, (radial averaging).

7. Change the value of fitting parameter, A until $\overline{\varepsilon_{\mathrm{G}}}=$ overall gas holdup. If there is no good agreement then repeat steps 2) through 6) until convergence criterion is achieved $\left(\overline{\varepsilon_{\mathrm{G}}}=\right.$ overall gas holdup $)$.

At the studied operating conditions and CT levels, the flow pattern is expected to be fully developed (i.e. L/D > 1) (Degaleesan et al., 1997). The assumptions made in this methodology would be used without much significant error. Therefore, all the obtained CT data has been processed using this methodology. 


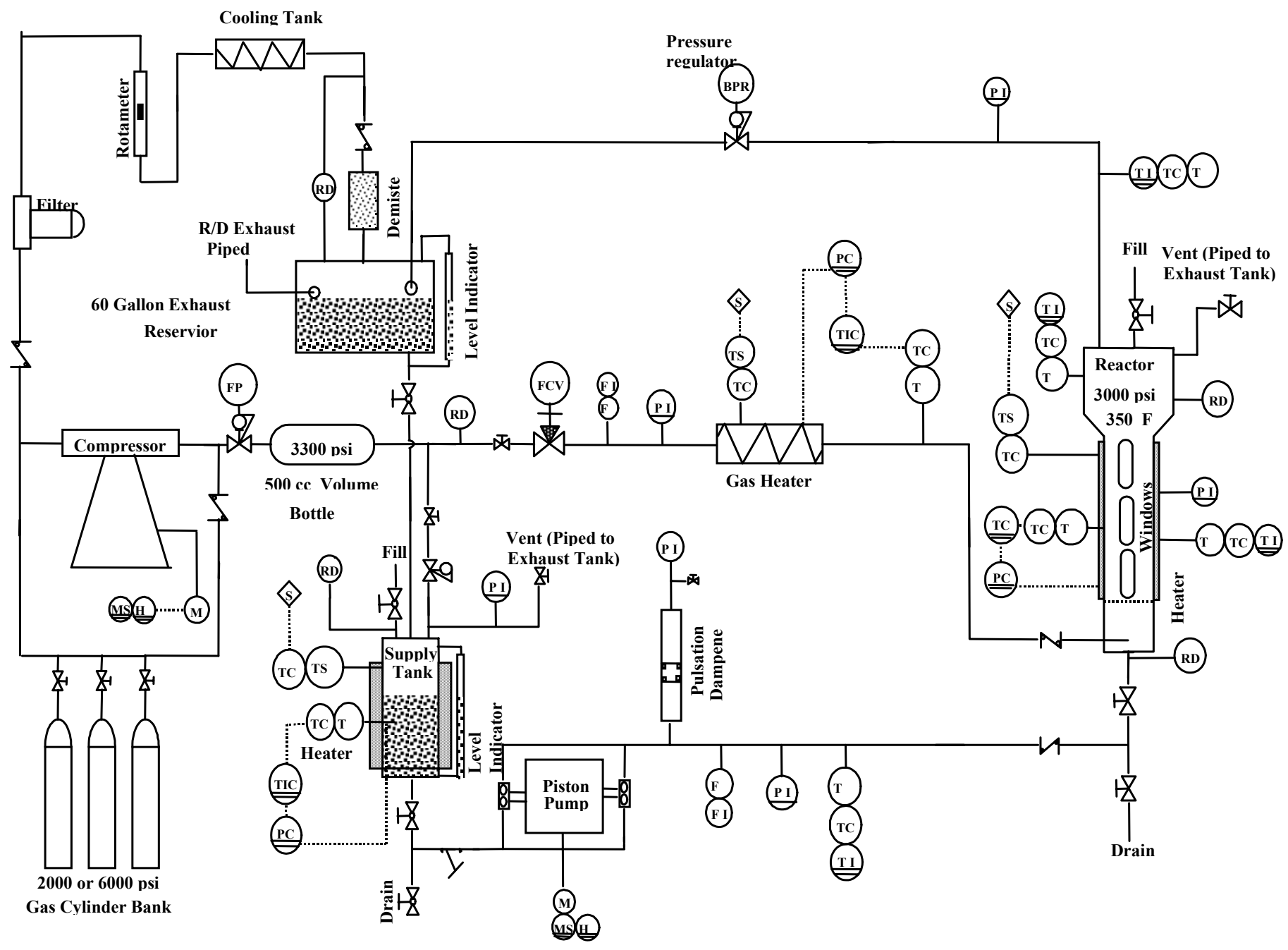

Figure 3.1. Schematic diagram for high pressure and high temperature slurry bubble column. 


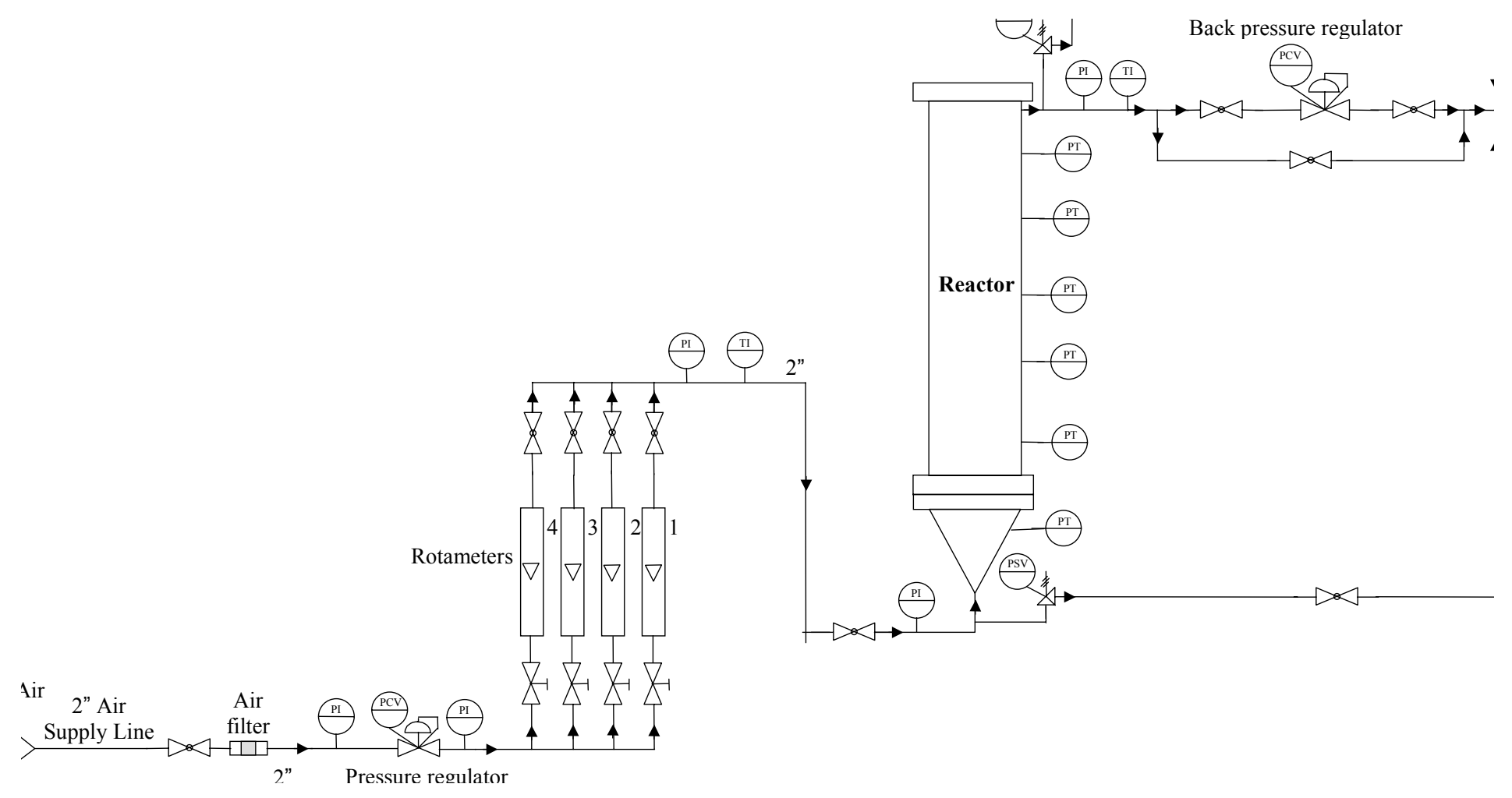

Figure 3.2. Gas flowsheet for the high pressure 6 inch diameter bubble column 


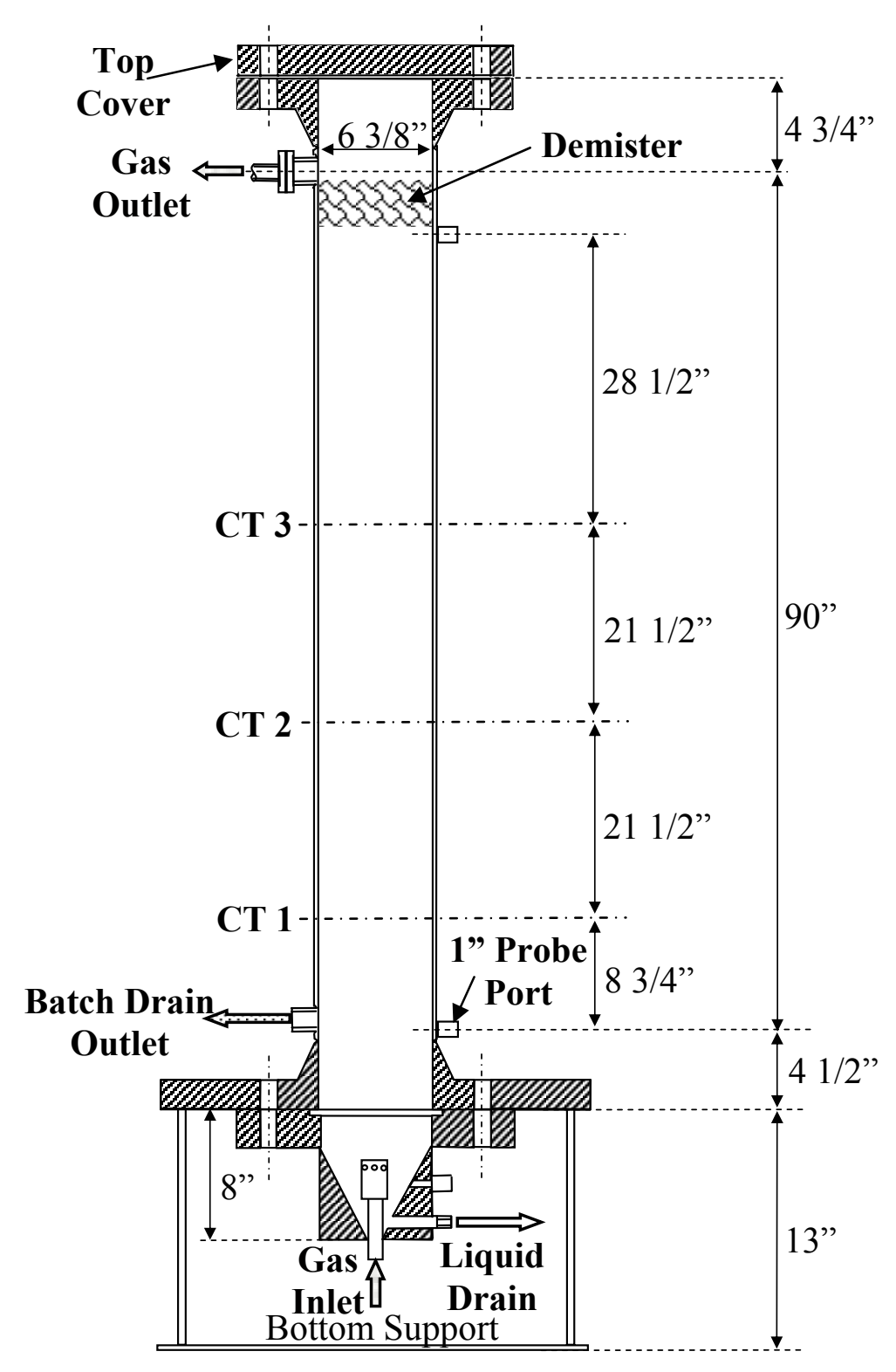

Figure 3.3: Bubble column reactor of 6 inch without ports used for CARPT/CT measurements. CT1, CT2, and CT3 represent the scan levels used in this investigation. 


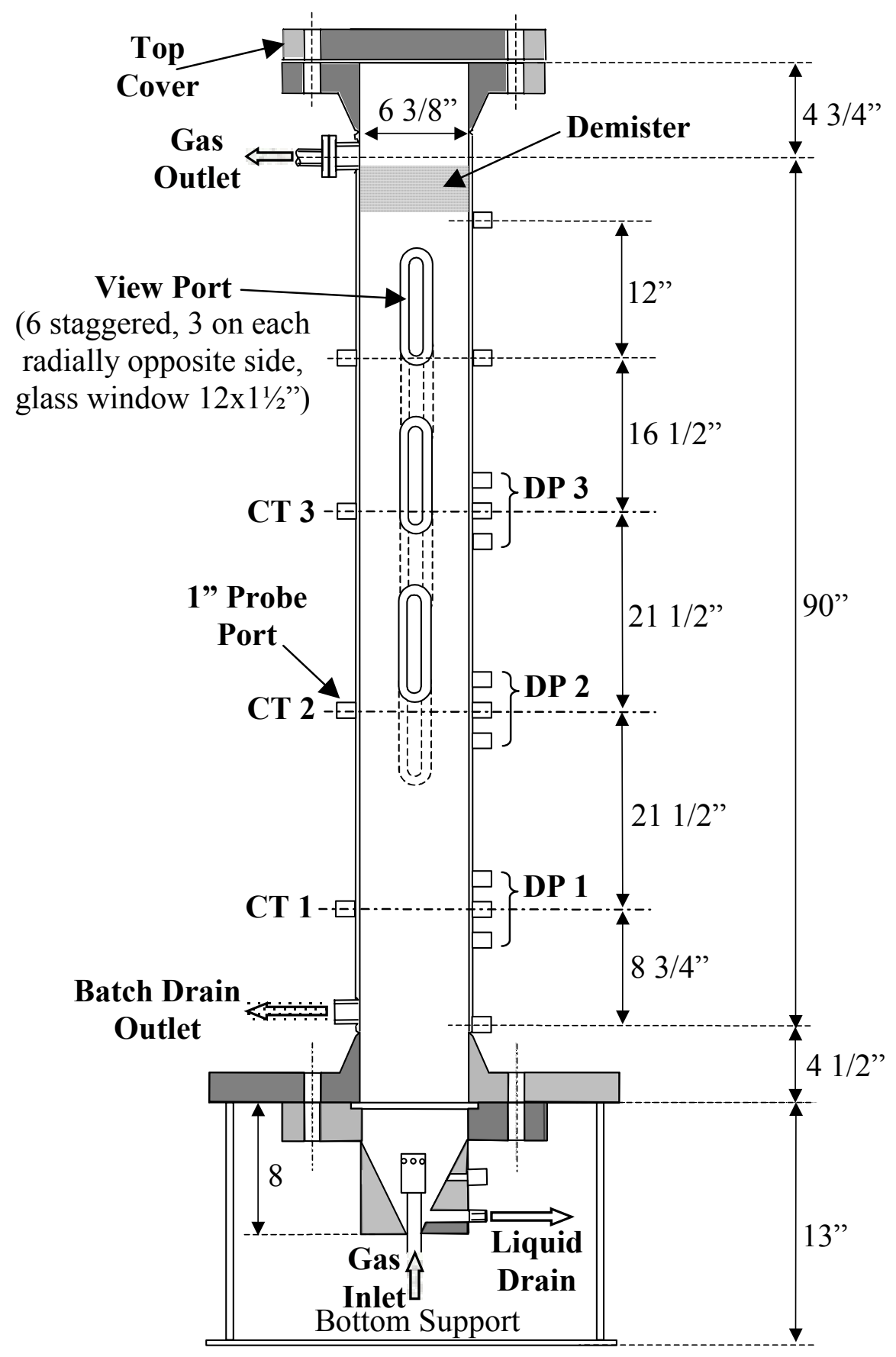

Figure 3.4: Bubble column reactor of 6 inch with ports used for overall gas holdup and DP measurements. CT1, CT2, and CT3 represent the scan levels used in this investigation. 


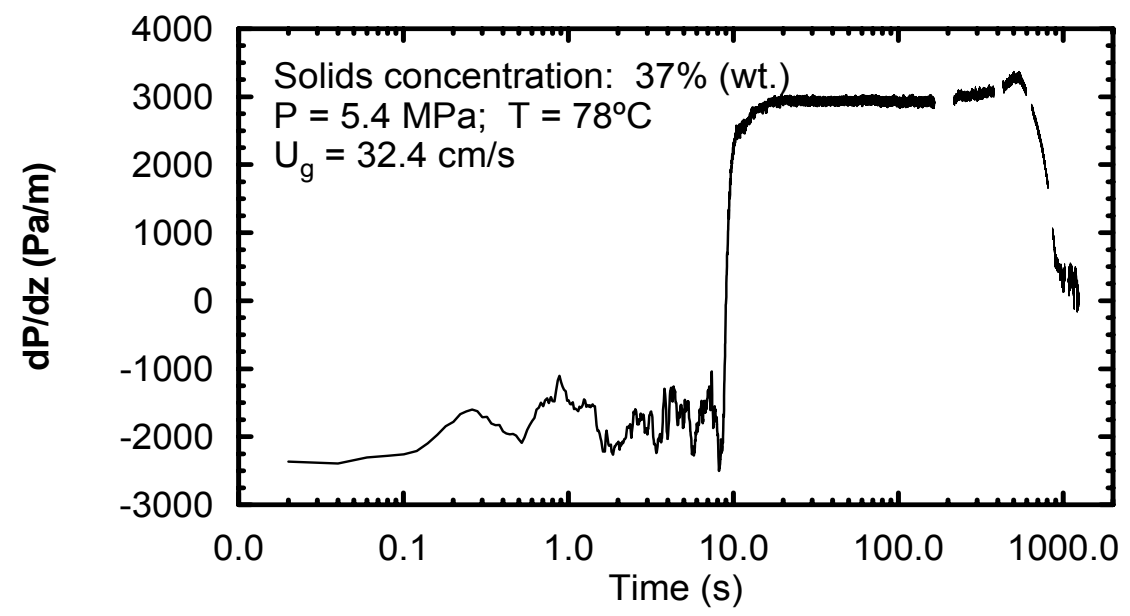

Figure 3.5: Typical variation of dynamic pressure gradient with time during the bed disengagement process.

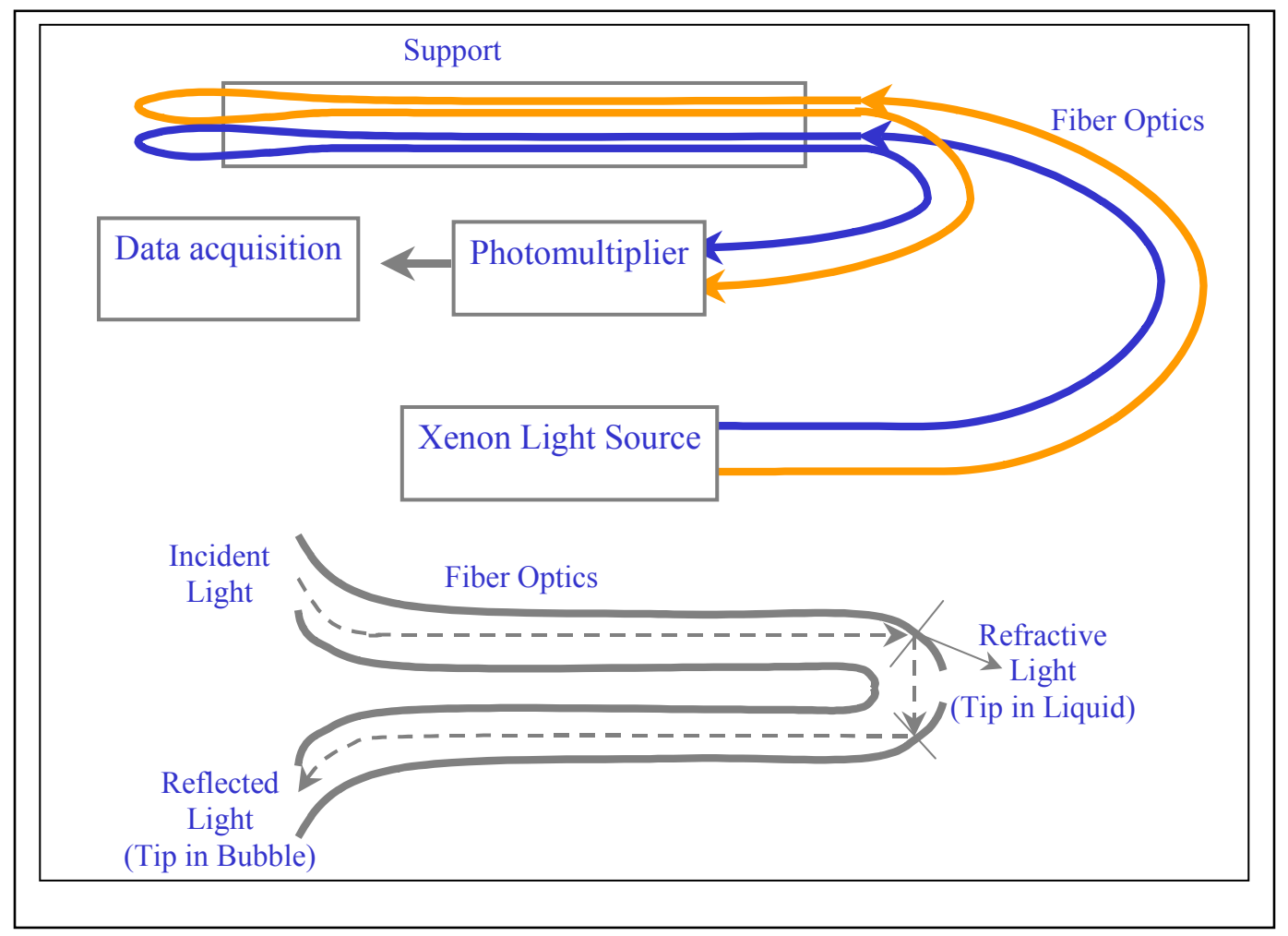

Figure 3.6: Schematic diagram of the U-shaped optical fiber probe 


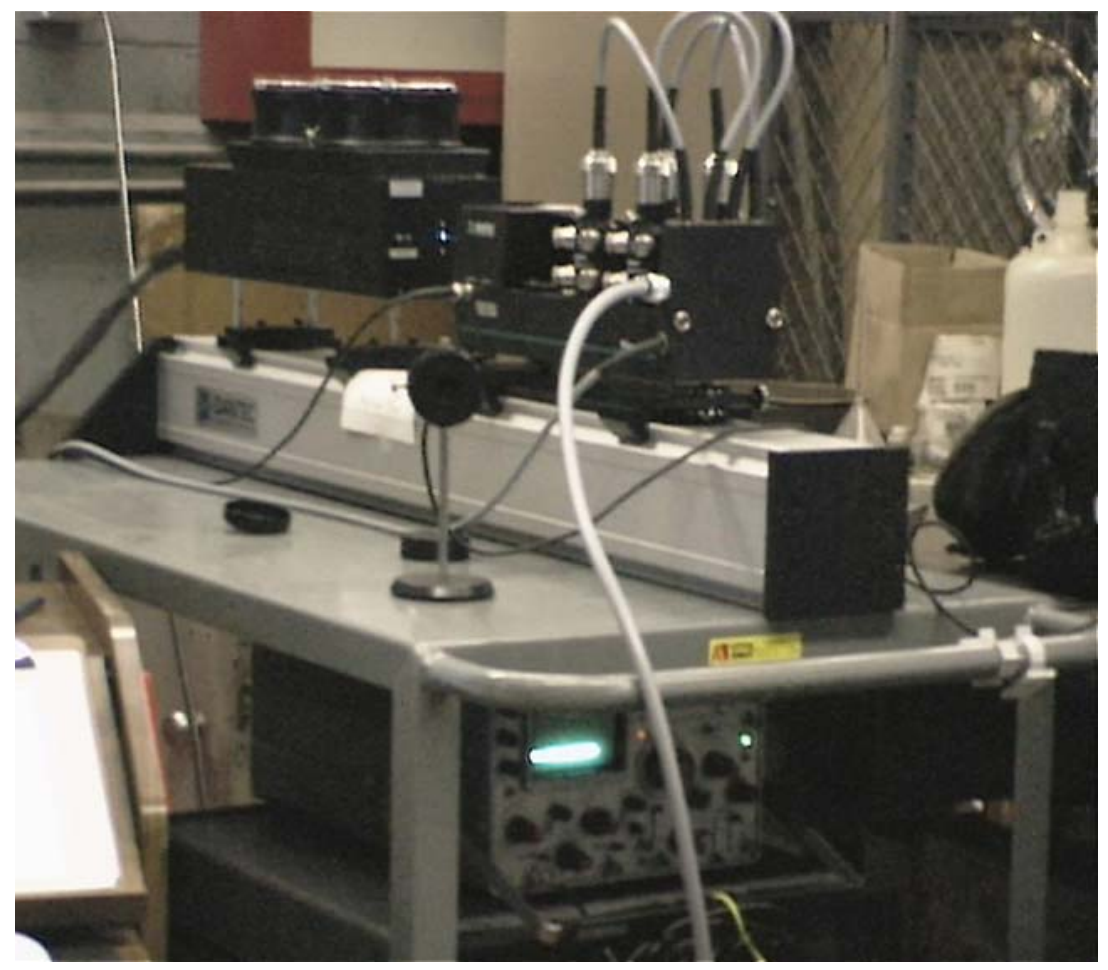

Figure 3.7: Laser Doppler Anemometer Setup. 


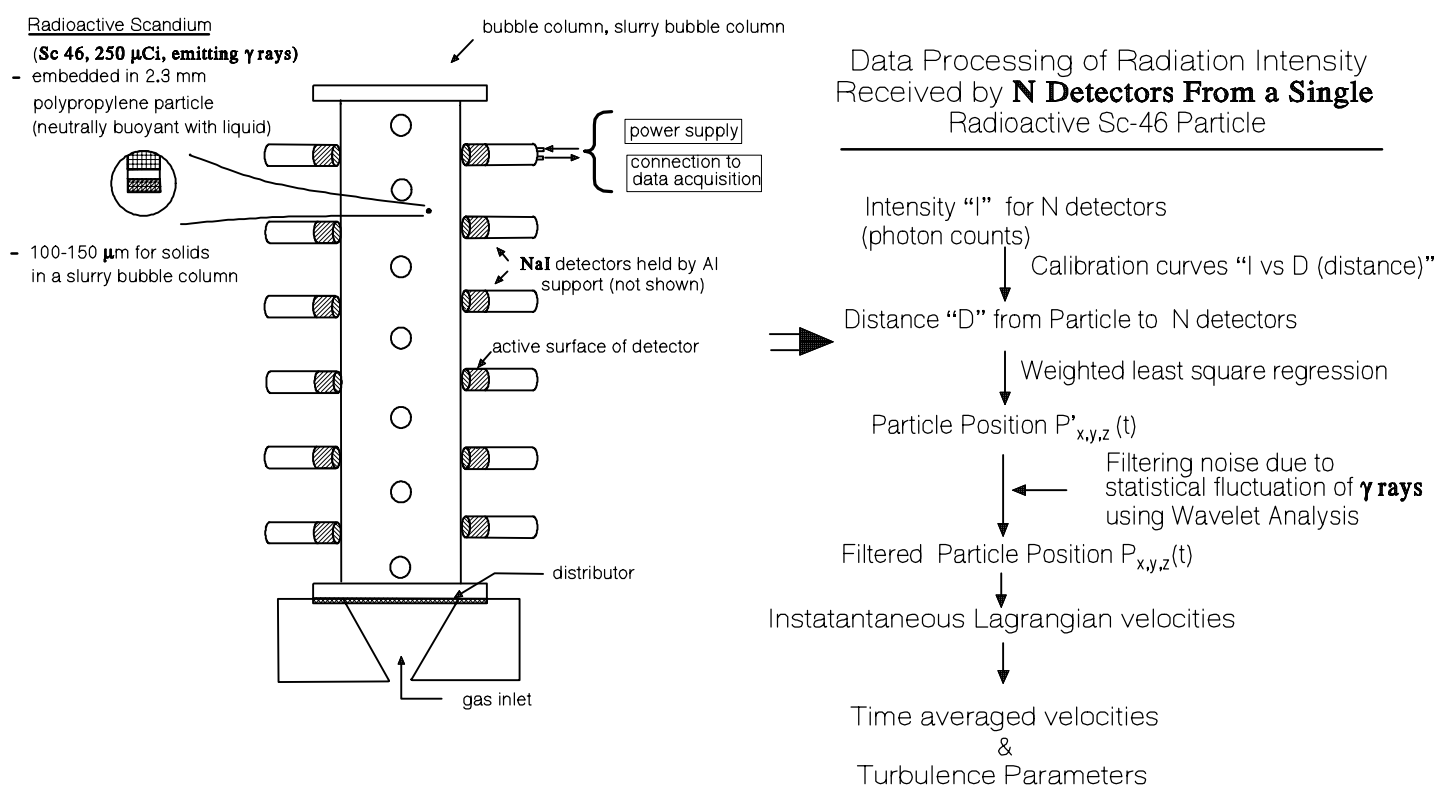

Figure 3.8: Configuration of the CARPT experimental setup. 


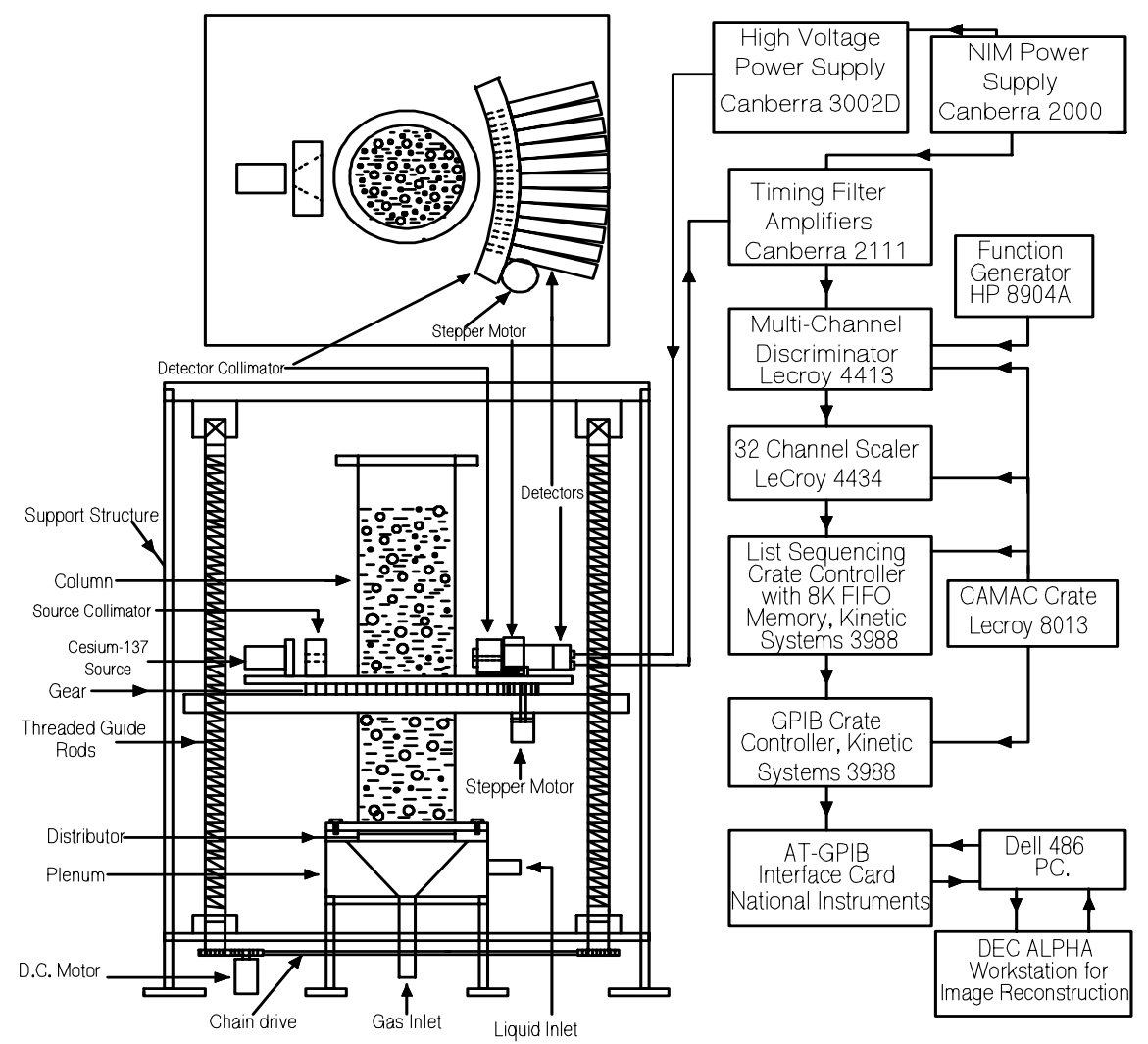

Figure 3.11: Configuration of the CT experimental setup (Kumar, 1994). 


\section{RESULTS AND DISCUSSIONS}

The following is the summary of the results for the investigations made during the course of this project. Some of the other results and findings are reported in the published manuscripts that are attached as appendices.

\subsection{Hydrodynamics measurements in 2 inch column using DGD, $\Delta P$ Fluctuation, Fiber Optic Probe, and LDA}

This work has been carried out at Ohio State University. The liquid and gas phase used for these experiments are Norpar 15 (density $=0.772 \mathrm{~g} / \mathrm{cc}$, viscosity $=2.13 \mathrm{cPs}$, surface tension $=26.7 \mathrm{dynes} / \mathrm{cm}$ at ambient temperature and pressure) and nitrogen. The solid phase was glass beads of $150 \mu \mathrm{m}$. All the experiments have been operated in a batch liquid mode. The gas distributor is comprised of 19 triangular pitched holes of $0.45 \mathrm{~mm}$ diameter each and \% open area is 0.156 . The static liquid level is maintained at $50 \mathrm{~cm}$ above the distributor. Pliolite particles of $1.02 \mathrm{~g} / \mathrm{cm} 3$ in density with a size range of $20-50 \mathrm{~mm}$ are used as the liquid tracer for LDA technique.

\subsubsection{Overall gas holdup using DGD}

Dynamic Gas Disengagement (DGD) experiments have been performed to study the effect of operating pressure and solids loading on the overall gas holdup in 2 " column.

Figures 4.1 and 4.2 show the effect of pressure and solids loading on overall gas holdup. At low superficial gas velocities, the effect of operating pressure on the gas holdup is less compared to high superficial gas velocities. The influence of pressure has been found to be significant at higher superficial gas velocities. As can be seen in Figure 4.1, overall gas holdup is approximately double at $2.38 \mathrm{MPa}$ relative to ambient pressure at superficial gas velocities higher than $20 \mathrm{~cm} / \mathrm{s}$. The presence of solids provides additional effects on the overall gas holdup. The gas holdup at high solids loading decreases considerably with an increase in pressure. The effect of addition of solids is less at low solids loading while it has been found to be significant at high solids loading, especially for liquid with high viscosity. Figure 4.2 summarizes the effect of solids loading on overall gas holdup at various isobaric conditions. The influence of solids loading on gas holdup is insignificant at ambient pressure while the significant effect is observed at high pressure. In general, overall gas holdup decreases with an increase in solids loading, particularly at high operating pressure. The presence of solids in three-phase system results in formation of larger bubbles due to increase in coalescence rate, which reduces overall gas holdup. Therefore, it can be concluded that an increase in operating pressure and decrease in the solids loading can improve the overall gas holdup.

\subsubsection{Prediction of regime transition using $\triangle P$ fluctuations}

Figure 4.3 shows the effect of solids loading and operating pressure on the flow regime transition in 2" column using nitrogen-Norpar 15-glass beads $(150 \mu \mathrm{m})$ system. The increase in pressure causes formation of smaller uniform sized bubbles, and hence the flow regime tends to be in homogeneous regime. This delays the regime transition. The 
addition of solids loading, on contrast, reduces transition velocity due to increase in pseudo-viscosity of slurry, which increases large bubble population.

\subsubsection{Bubble Size and Bubble Rise Velocity}

Bubble size increases significantly with an increase in solids loading at ambient pressure and the effect is inhibited at elevated pressure. At ambient pressure, the bubble size distribution is found to be wide. The maximum bubble size increases with an increase in superficial gas velocity. The maximum bubble size is about $2.6 \mathrm{~cm}$ at a gas velocity of $37 \mathrm{~cm} / \mathrm{s}$. The prolonging of the bubble formation to its maximum stable size largely exists due to internal circulation of the gas. The internal circulation velocity is of the same order of magnitude as the bubble rise velocity. A centrifugal force is, induced by this circulation, pointing towards the bubble surface in outer direction. This force can suppress the disturbances at the gas-liquid interface and thereby stabilizing the interface. On other hand, the centrifugal force can also disintegrate the bubble as it increases with an increase in bubble size. The bubble breaks up when the centrifugal force exceeds the surface tension force, especially at high pressures where the gas density is high. A much smaller bubble size is observed at high pressure conditions compared with ambient pressure conditions, indicating that pressure has a significant effect on the breakage of the large bubbles. A narrower bubble size distribution is also observed under high pressure conditions. An increase in solids loading increases the maximum bubble size slightly. Figures 4.4 and 4.5 show the effects of pressure and solids loadings on bubble size and bubble rise velocity at superficial gas velocity of $30 \mathrm{~cm} / \mathrm{s}$ and operating pressure of 1.78 $\mathrm{MPa}$. The bubble rise velocity decreases with an increase in pressure for a given solids loading. In general, the addition of solids can reduce bubble rise velocity drastically. Further, due to the dominant role of the large bubbles in determining the gas holdup, the increase in bubble size due to the presence of particles explains the decrease in gas holdup as the solids loadings increases.

\subsubsection{Averaged liquid velocity and Reynolds stresses measured by LDA using Norpar 15-nitrogen system}

In order to let the laser beam to penetrate through the sight glass, only gas-liquid (Norpar 15-nitrogen) system and superficial gas velocity up to $8 \mathrm{~cm} / \mathrm{s}$ have been used in this investigation. Figure 4.6 shows the average liquid velocity at different superficial gas velocities and at ambient pressure. The average liquid velocity is higher in the center region of the column. A down flow is observed in the wall region due to circulation. The inversion of velocity takes place at around $\mathrm{r} / \mathrm{R} \sim 0.7$. The tangential and axial Reynolds normal stresses are illustrated in Figure 4.7 and 4.8, respectively. Both the tangential and axial Reynolds normal stresses are the lowest at the wall and the value levels off as the radial location moves toward the center. The center has slightly lower tangential and axial Reynolds normal stresses due to the swirling motion of the central bubble stream. In the center of the column, the flow is more frequently upward, whereas in the vortical region, the flow dynamically changes from upward to downward depending on the location of the central bubble stream. The flow in this region therefore experiences large fluctuations in the axial component of the liquid velocity, leading $\langle\mathrm{v}$ 'v' $\rangle$ to peak closer to the center 
of vortical structures rather than in the center where the motion is primarily directed upward. Here, turbulent parameters are measured upto $8 \mathrm{~cm} / \mathrm{s}$ because in order to let the laser to penetrate through the sight glass, one has to keep superficial gas velocities low enough. 
Solids loading $0 \% \mathrm{wt}$

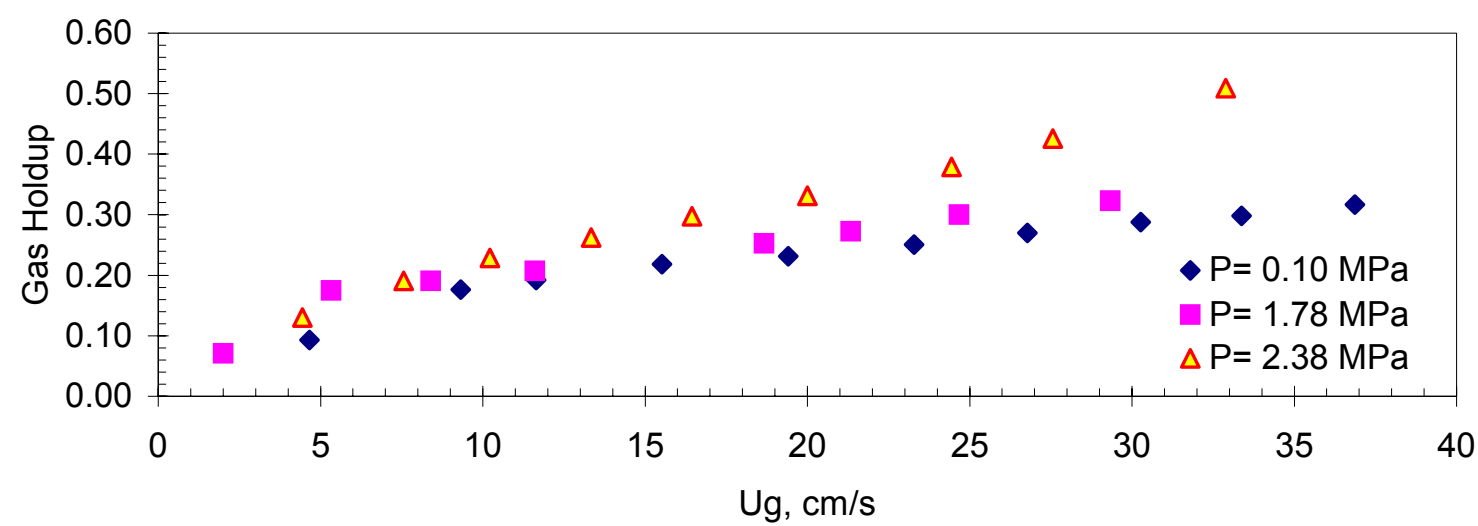

Solids loading $20 \% \mathrm{wt}$

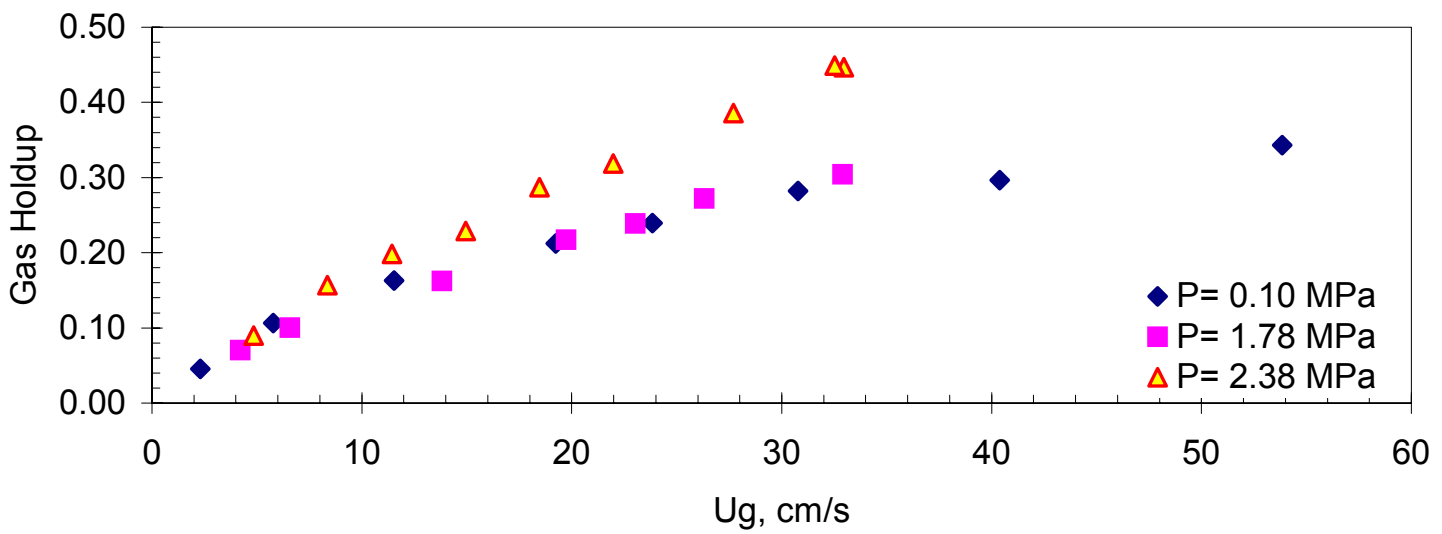

Solids loading $40 \%$ wt

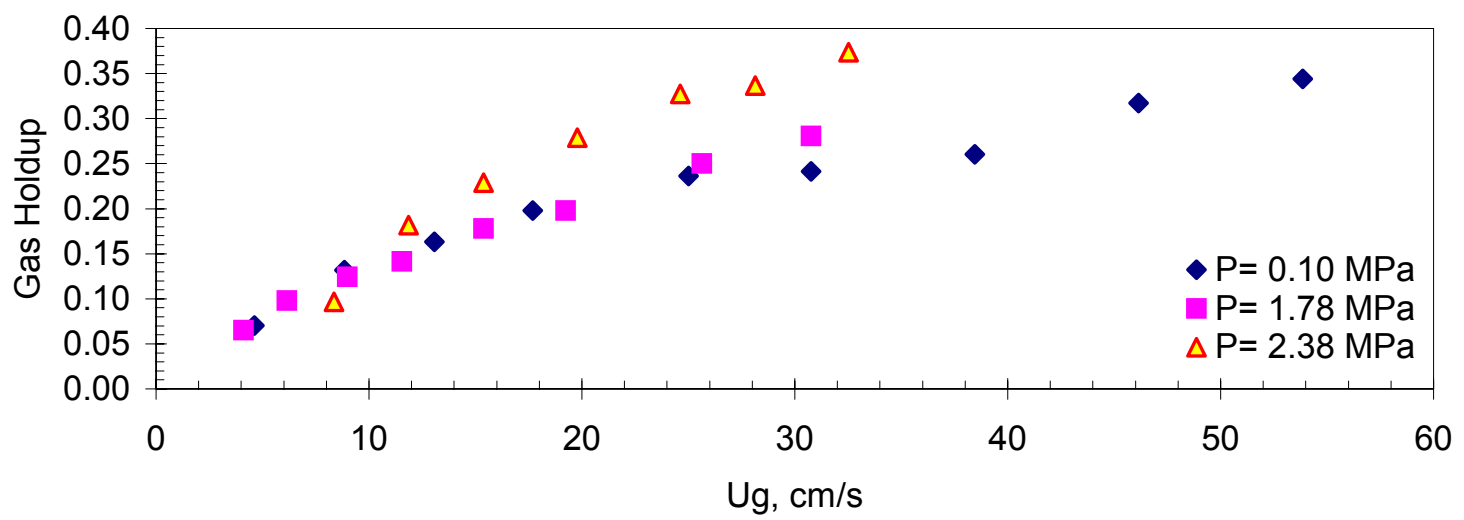

Figure 4.1: Effect of pressure on overall gas holdup (Nitrogen - Norpar 15 - 150 $\mathrm{mm}$ glass beads) at various solids loading 
Pressure $=0.1 \mathrm{MPa}$

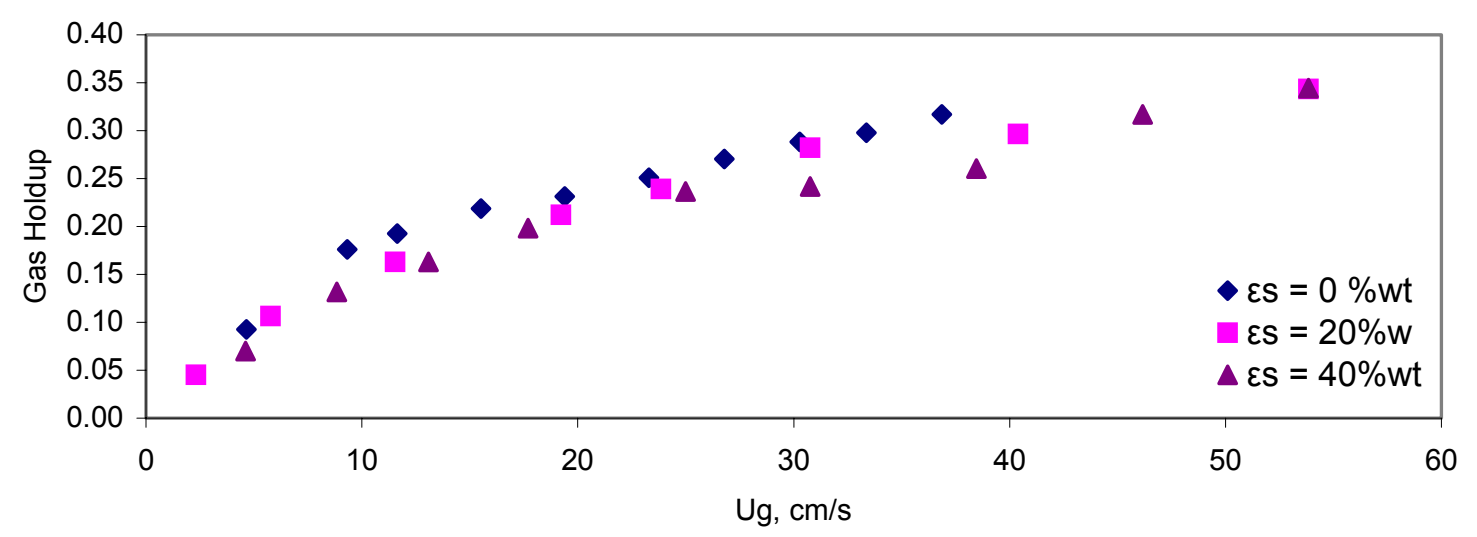

Pressure $=1.78 \mathrm{MPa}$

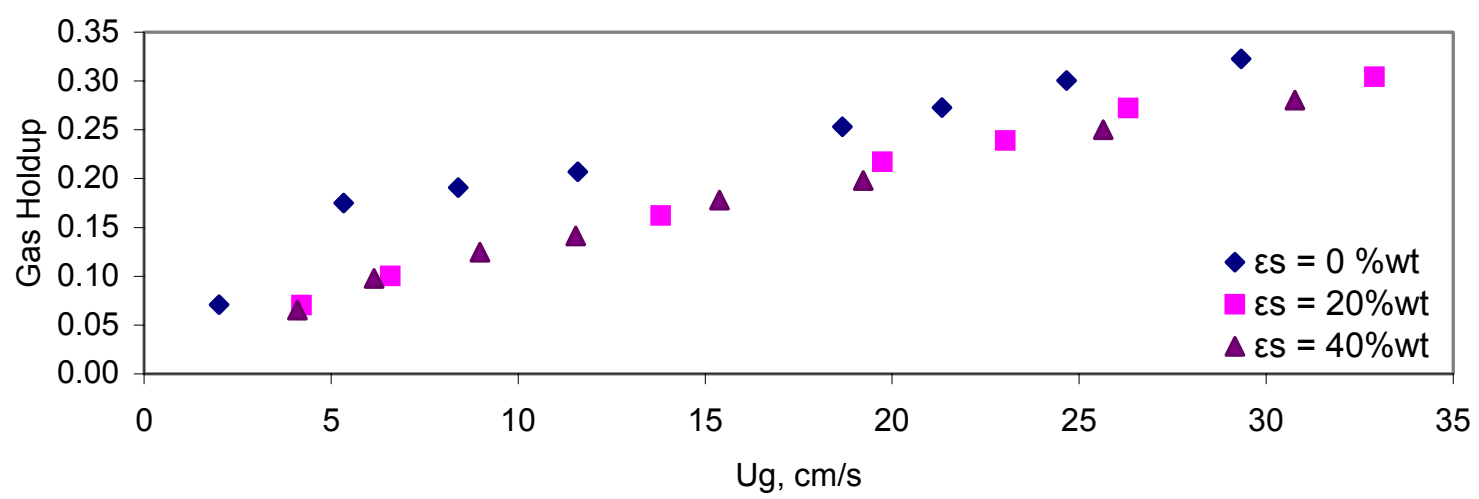

Pressure $=2.38 \mathrm{MPa}$

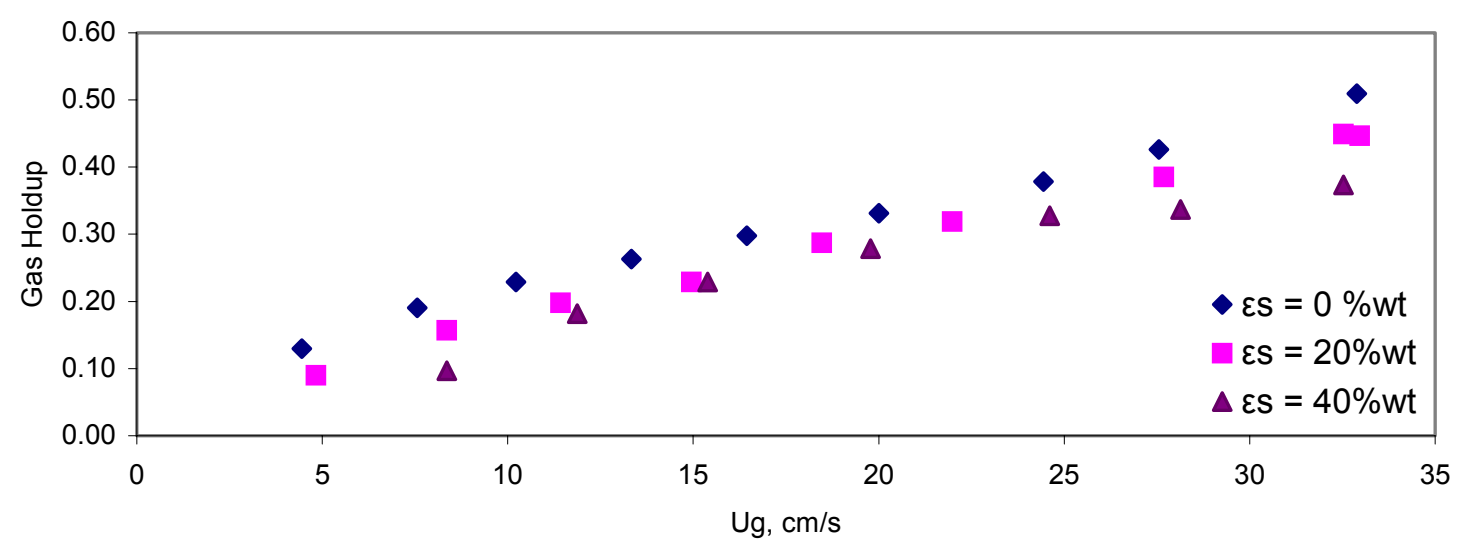

Figure 4.2: Effect of solids loading on gas holdup (Nitrogen - Norpar 15 - 150 $\mu \mathrm{m}$ glass beads) at various operating pressures 
a) Effect of Solids Loading

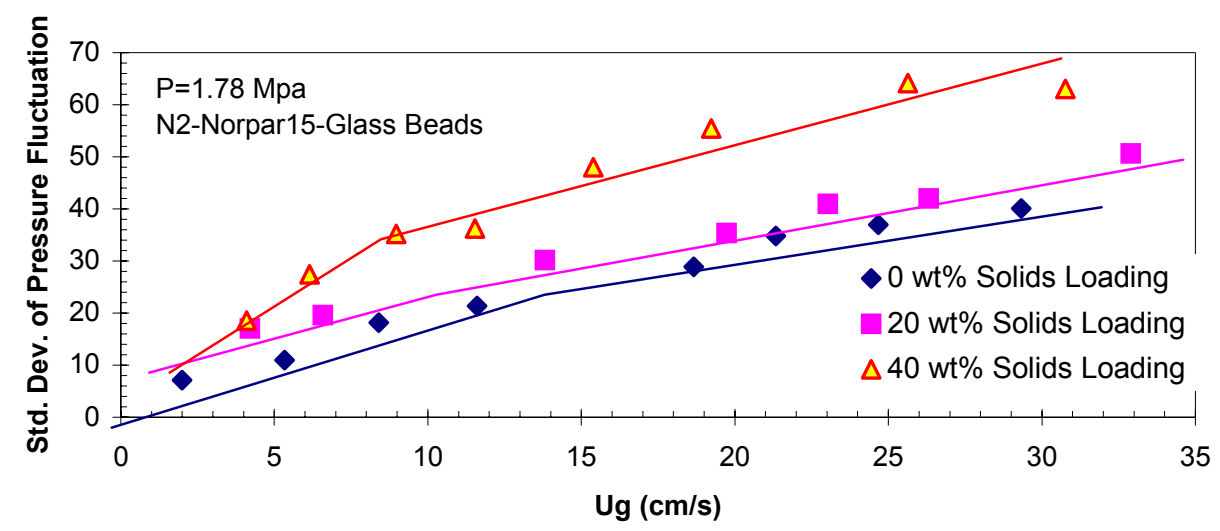

b) Effect of Operating Pressure

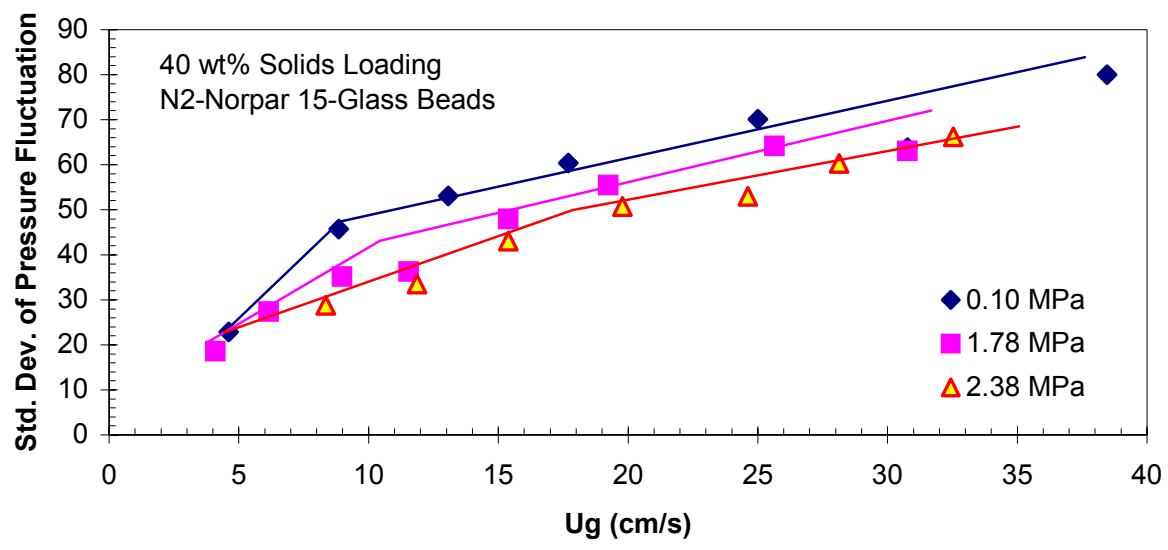

Figure 4.3: Effect of a) solids loading and b) operating pressure on regime transition using Nitrogen - Norpar $15-150 \mu \mathrm{m}$ glass beads in 2" column. The lines show the slope of standard deviation of pressure fluctuation (Lin et al., 1999). 

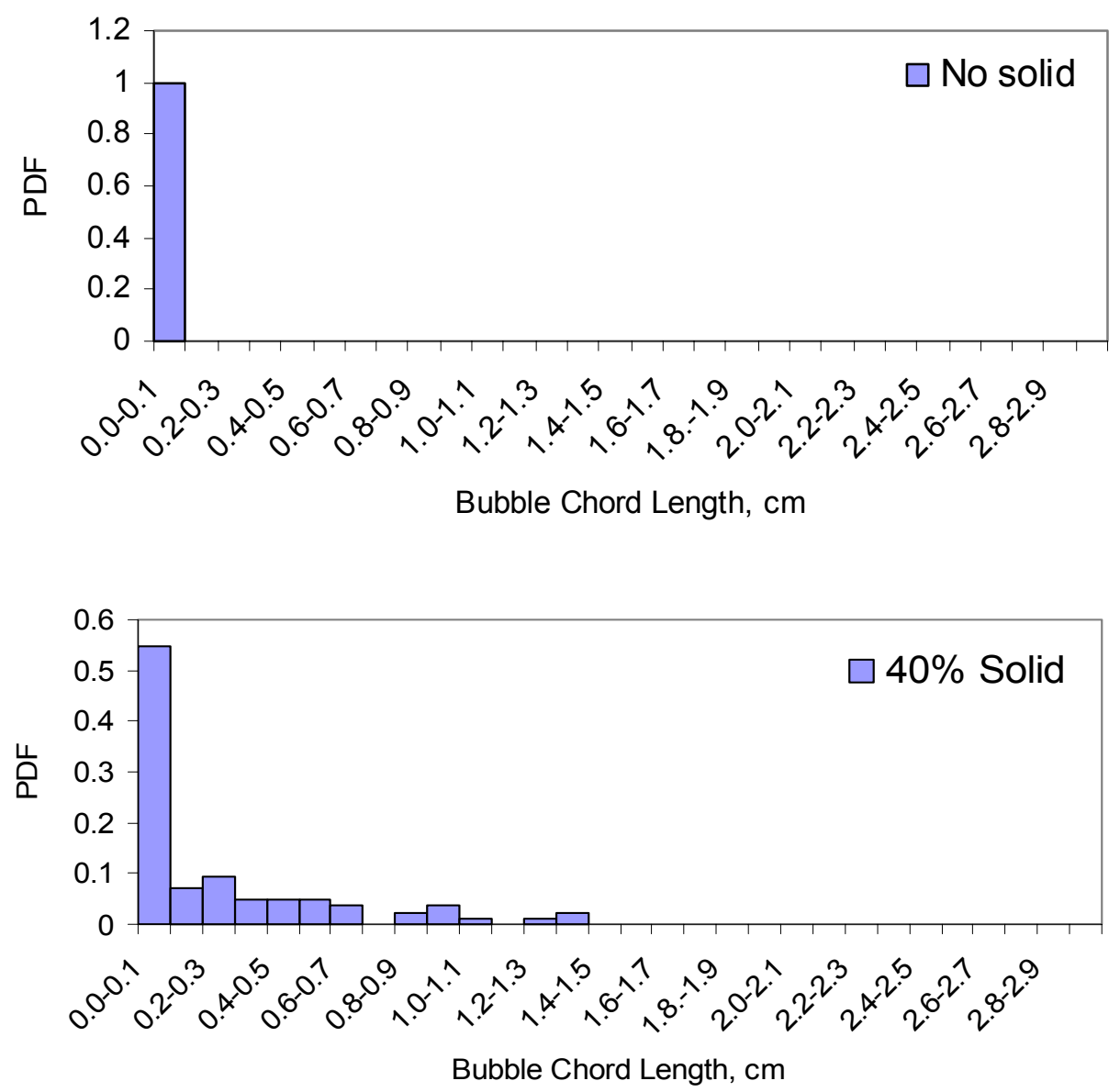

Figure 4.4: Effect of solids loading on bubble size using Nitrogen - Norpar $15-150 \mu \mathrm{m}$ glass beads in 2" column at $\mathrm{Ug}=30 \mathrm{~cm} / \mathrm{s}, \mathrm{P}=1.78 \mathrm{MPa}$ 

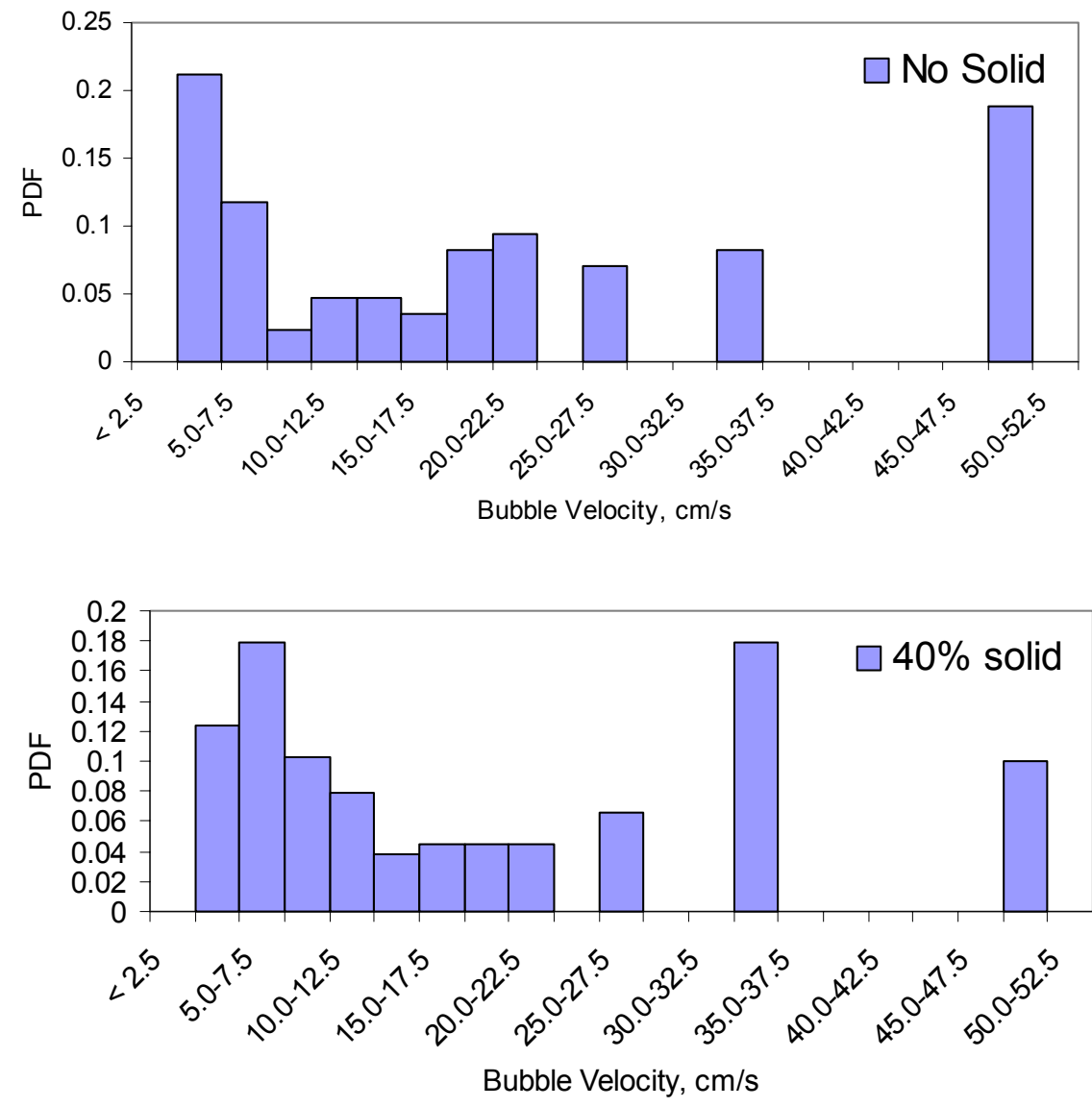

Figure 4.5: Effect of solids loading on bubble rise velocity using Nitrogen - Norpar 15 $150 \mu \mathrm{m}$ glass beads in 2 " column at $\mathrm{Ug}=30 \mathrm{~cm} / \mathrm{s}, \mathrm{P}=1.78 \mathrm{MPa}$ 


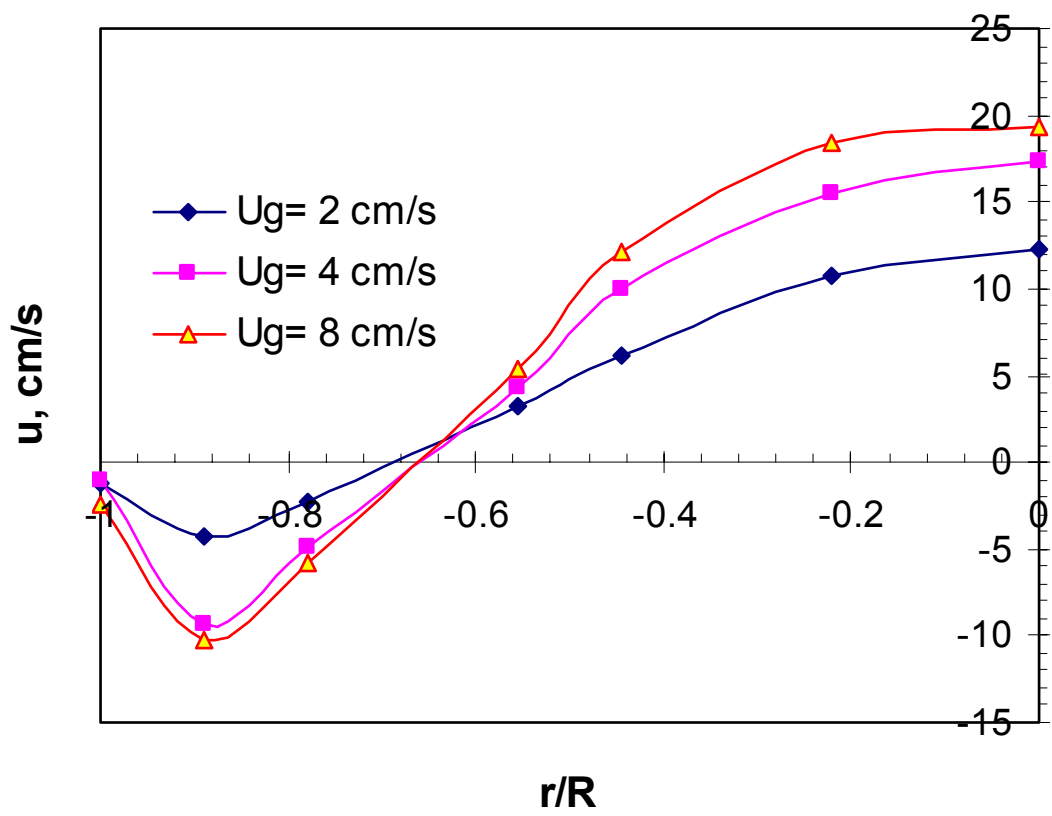

Figure 4.6: Averaged liquid velocity measurement at various superficial gas velocities at ambient pressure

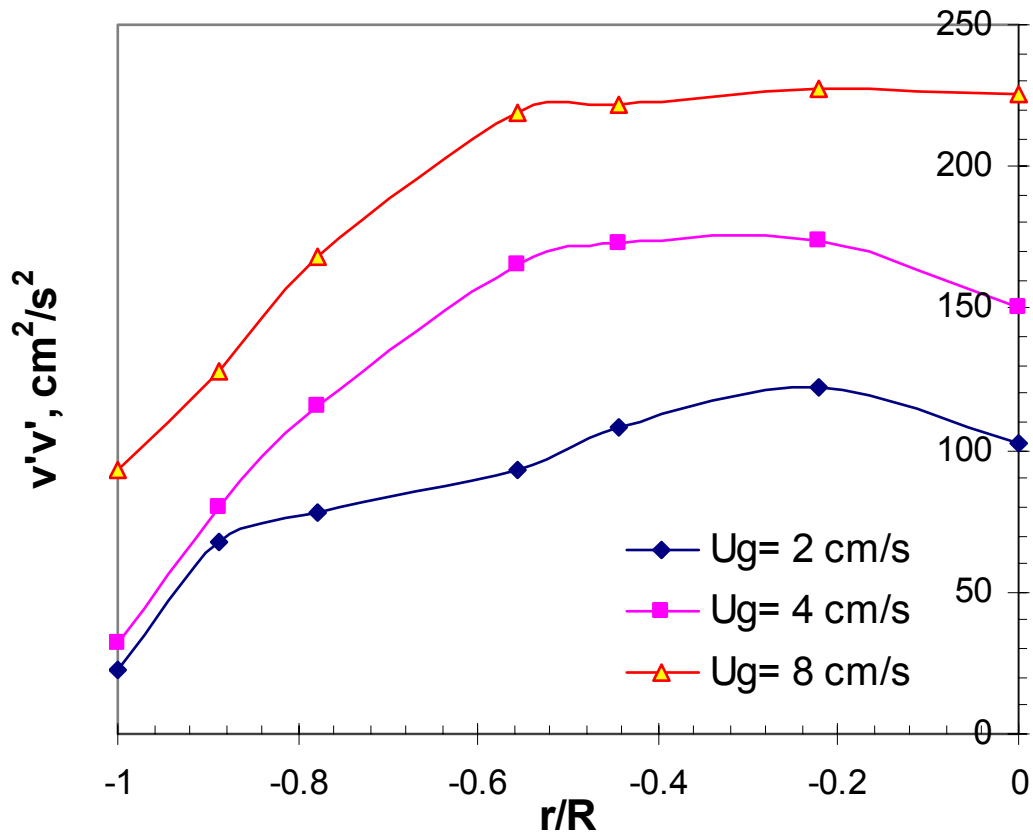

Figure 4.7: Tangential Reynolds normal stress at various superficial gas velocities at ambient pressure 


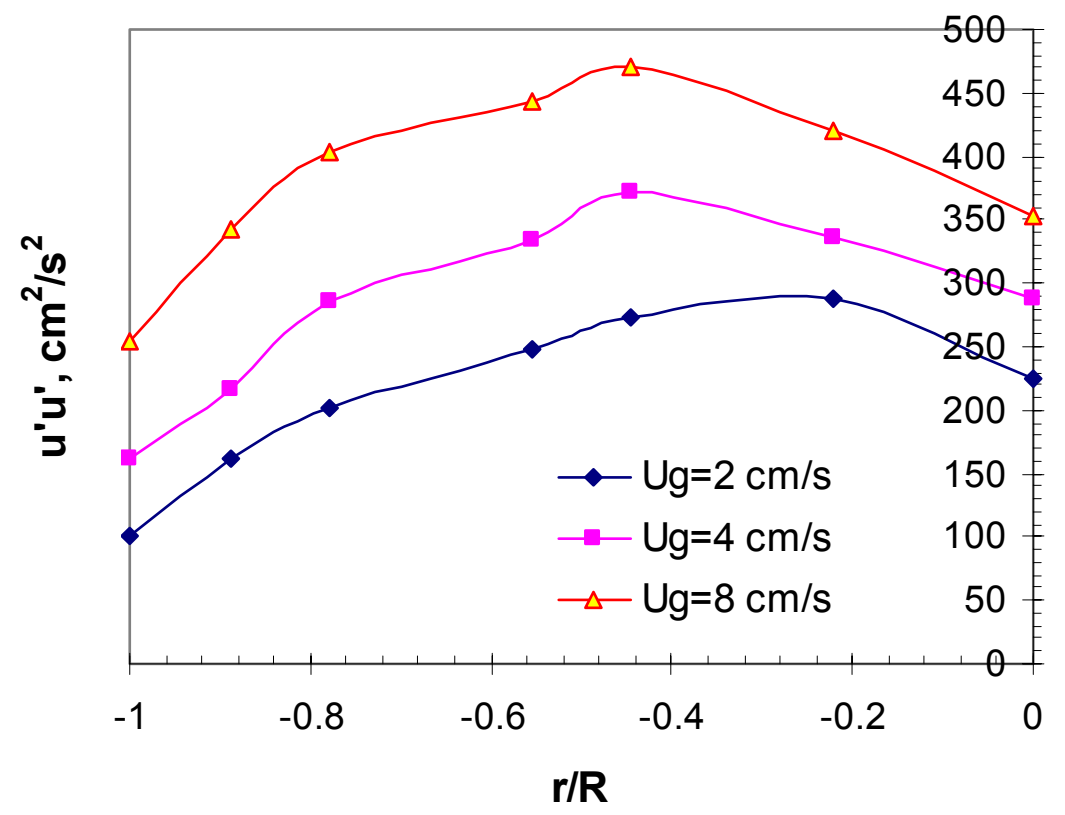

Figure 4.8: Axial Reynolds normal stress at various superficial gas velocities at ambient pressure 


\subsection{Hydrodynamics Measurements in 6 inch column using CARPT and CT}

The three-phase holdups distribution presented in this section has been computed by the newly proposed CT/Overall gas holdup methodology. The findings of CARPT/CT are in agreement with those obtained by LDA, optical probe, and DGD performed at OSU.

\subsubsection{Results of CT (gas holdup profile)/CARPT (solids axial velocity profile and turbulent parameters) Using Air-Water-Glass Beads System}

\subsection{1 a) Effect of Superficial Gas Velocity}

The reported literature suggests that an increase in superficial gas velocity increases the gas holdup and the liquid/solids velocity in both two- and three-phase bubble columns operated at atmospheric pressure (Degaleesan, 1997, Sannaes, 1997). The same effect of superficial gas velocity on radial gas holdup and solids velocity profiles at atmospheric pressure is observed in slurry bubble columns during this work. Figures 4.9 and 4.10 illustrate the effect of superficial gas velocity on radial gas holdup profiles and solids axial velocity profiles at atmospheric pressure. An increase in superficial gas velocity from 8 to $45 \mathrm{~cm} / \mathrm{s}$ increases the centerline gas holdup from 0.3 to 0.55 while the centerline solids axial velocity increases from 24.59 to $48.21 \mathrm{~cm} / \mathrm{s}$. An increase in solids centerline axial velocity with an increase in superficial gas velocity is compensated with a larger negative axial velocity $(-18.0$ to $-26.5 \mathrm{~cm} / \mathrm{s})$ at the wall, preserving the zero net solids flux. This results in an increase in the solids recirculation velocity in slurry bubble columns as the superficial gas velocity increases. The inversion point, where axial solids velocity become zero, occurs at $\phi_{0}=0.65-0.70$. It was found that, the inversion point shift slightly towards the center of the column with an increase in superficial gas velocity.

The solids shear stress is proportional to the radial gradient of solids axial velocity. As there is an increase in solids axial velocity with an increase in superficial gas velocity (Figure 4.11), the shear stress should increase with an increase in superficial gas velocity. As shown in Figure 4.11, shear stress profiles exhibit maximum at $\mathrm{r} / \mathrm{R} \approx 0.5$ while at the wall and in the center of the column, shear stress values are close to zero.

The system becomes more turbulent with an increase in superficial gas velocity, which is reflected in an increased turbulent kinetic energy (TKE) (Figure 4.12) and eddy diffusivity profiles (Figure 4.13). Turbulent kinetic energy (TKE) profiles exhibit maximum values in the center of the column and decrease towards the column wall (Figure 4.12$)$. The radial eddy diffusivity profiles $\left(D_{\mathrm{rr}}\right)$ are qualitatively very similar to the shear stress profiles and exhibit maxima at $r / R=0.4-0.5$ while at the wall and in the center of the column diffusivity values are close to zero. The magnitude of radial diffusivity $\left(D_{r r}\right)$ has been very low compared to axial diffusivity $\left(D_{z z}\right)$ as shown in Figure 4.13. The axial eddy diffusivity profiles exhibit maxima close to the axial velocity inversion point at $\mathrm{r} / \mathrm{R} \approx 0.65$ (Figure $4.13 \mathrm{a}$ ). The centerline and the wall axial eddy diffusivities are typically between 50 and $80 \%$ of the maximum axial eddy diffusivity value. 


\subsection{1 b) Effect of Operating Pressure}

An increase in pressure increases bubble break-up rate, which results in generation of smaller bubbles and thereby increases gas holdup. Therefore, bubble column systems operated at higher pressures are characterized by larger gas holdup profiles (CREL Report, 2000a). The higher gas holdup and smaller size bubbles entrain the suspension of solids and liquid more effectively, which causes higher liquid and solids axial velocity profiles and therefore higher solids and liquid recirculation. This explanation has not been so far supported by experimental findings. However it is supported by the present CARPT solids velocity measurements in slurry systems and liquid velocity measurements in high pressure G-L bubble column systems.

The effect of increased pressure that results in higher gas holdup and solids axial velocity profiles is illustrated in Figures 4.14 and 4.15. The comparison of the gas holdup and the solids axial velocity profiles at different conditions shows that, the effect of pressure on gas holdup and solids axial velocity profiles is as strong as the effect of superficial gas velocity. The shear stress is proportional to the radial gradient of axial velocity and therefore higher solids axial velocity profiles result in higher shear stress profiles. It has been shown that an increase in superficial gas velocity increases the solids axial velocity profiles. As an increase in pressure increases solids axial velocity, the higher shear stress profile has been observed at high pressure conditions (Figure 4.16). The comparison of Figures 4.11 and 4.16 leads to a conclusion that, the effect of pressure on the shear stress profiles is significantly smaller compared to the effect of superficial gas velocity. The shear stress profiles in high pressure systems are qualitatively similar to the profiles in systems operated at atmospheric pressure, with the maximum location at $r / R \approx 0.5$.

It has been shown by several authors that, an increase in pressure results in smaller and more uniform size of the bubbles. The smaller bubbles rise without much interaction even at considerable superficial gas velocities causing a delay in the flow regime transition (Lin et al, 1998; Luo, et al., 1999). In the high pressure systems, even in the churn turbulent flow regime, bubbles of small and narrow size distribution interact to a much smaller extent than the larger bubbles at atmospheric pressures (Wilkinson, 1991; Luo et al., 1999). Thus at the same superficial gas velocity, the system at higher pressures are less turbulent compared to atmosphere pressure system. Therefore flow at higher pressure has been characterized by lower TKE (Figure 4.17) and eddy diffusivity (Figure 4.18) profiles.

Hence, the effect of pressure appears to be much weaker than the effect of superficial gas velocity on the TKE and eddy diffusivity profiles. The effect of increase in pressure on turbulent parameters is much less compared to the effect of superficial gas velocity, both qualitatively and quantitatively. 


\subsubsection{Results of CT (gas holdup profile) and CARPT (liquid axial velocity and turbulent parameters) using air-water system [Ong, 2003]}

\subsection{2 a) Effect of Superficial Gas Velocity:}

The effect of superficial gas velocity on gas holdup and liquid axial velocity at atmospheric pressure is shown in Figures 4.19 and 4.20. An increase in superficial gas velocity increases gas holdup and liquid axial velocity as in air-water-glass beads system discussed earlier. The inversion point, at which liquid velocity becomes zero, for velocity profile in air-water system is found to be at $r / R=0.65$ similar to slurry systems. Also, turbulent kinetic energy and shear stress increases with an increase in superficial gas velocity, as illustrated in Figures 4.21 and 4.22, indicating greater turbulence in liquid phase at higher superficial gas velocity. An increase in superficial gas velocity from 30 $\mathrm{cm} / \mathrm{s}$ to $45 \mathrm{~cm} / \mathrm{s}$ increases centerline velocity by $20 \%$ while centerline TKE by $10 \%$. Figure 4.23 shows the effect of superficial gas velocity on axial and radial diffusivity profiles. The figure indicates that increasing superficial gas velocity increases the eddy diffusivity thereby enhances mixing in the system. The difference in eddy diffusivities for reported superficial gas velocities is much smaller than reported by Degaleesan (1997) at lower superficial gas velocities. Hence, as superficial gas velocity increases the diffusivities tend to level off.

As it was found in air-water-glass beads system, shear stress profile exhibit maxima at $\mathrm{r} / \mathrm{R} \approx 0.5$ while values near the wall and at the center are close to zero. The eddy diffusivity profiles also show the similar behavior as in air-water-glass beads system. The radial diffusivities show maxima between $\mathrm{r} / \mathrm{R}=0.4$ to 0.5 while axial diffusivities exhibit maxima near inversion point i.e. $\mathrm{r} / \mathrm{R}=0.65$.

\subsection{2 b) Effect of Operating Pressure:}

Figures 4.24 and 4.25 shows the effect of operating pressure on gas holdup and liquid axial velocity at superficial gas velocity of $45 \mathrm{~cm} / \mathrm{s}$. The gas holdup and consequently axial liquid velocity increase with increasing operating pressure. The increased operating pressure increases break up rate resulting into increased smaller bubbles population leading to higher gas holdup. Due to increase in liquid axial velocity with operating pressure, shear stress profile also increases in magnitude with an increase in operating pressure. The effect of pressure on shear stress has been shown in Figure 4.26.

Figure 4.27 shows the effect of pressure on turbulent kinetic energy at superficial gas velocity of $45 \mathrm{~cm} / \mathrm{s}$. The increased pressure results in decrease in TKE, probably due to the presence of higher small bubble population. Like TKE, axial and radial diffusivity profiles tend to reduce with an increase in operating pressure as shown in Figure 4.28. This trend can be attributed to reduction in bubble size with an increase in pressure.

At higher operating pressure, shear stress, axial and radial diffusivity profiles exhibit maxima at about $\mathrm{r} / \mathrm{R}=0.5,0.4-0.5$, and 0.65 respectively, similar to atmospheric pressure conditions. 


\subsubsection{Results of CT (gas and solids holdup profile) Using Air-Therminol LT-Glass Beads System}

\subsection{3 a) Effect of Superficial Gas Velocity:}

The effect of superficial gas velocity on gas holdup and solids holdup is shown in Figures $4.29 \mathrm{a}$ and b. Due to increase in overall gas holdup with superficial gas velocity, the magnitude of gas holdup profile also increases and the system tends to get into churnturbulent flow regime with increase in superficial gas velocity. The effect of superficial gas velocity on solids holdup profile is not much significant as compared to gas holdup, which would be due to the assumption of uniform cross-sectional solids loading in the CT/Overall gas holdup data reconstruction methodology discussed earlier in section 3.6. However, in the center region of the column $(\sim 0 \leq \mathrm{r} / \mathrm{R} \leq 0.5)$, solids holdup decreases slightly with the increase in superficial gas velocity, whereas at the wall region, the effect of superficial gas velocity diminishes.

\subsection{3 b) Effect of Operating Pressure:}

The effect of operating pressure on gas holdup and solids holdup profiles at superficial gas velocity of $14 \mathrm{~cm} / \mathrm{s}$ is shown in Figures $4.30 \mathrm{a}$ and $\mathrm{b}$. With an increase in pressure, the break up rate increases while coalescence rate decreases which leads to smaller bubble sizes and subsequently into an increase in gas holdup (Wilkinson, 1993). This results in higher gas holdup profile with an increase in operating pressure at the same superficial gas velocity. The solids holdup profile decreases with an increase in pressure. The effect of pressure on solids holdup profile is found to be less significant compared to the gas holdup profile. 


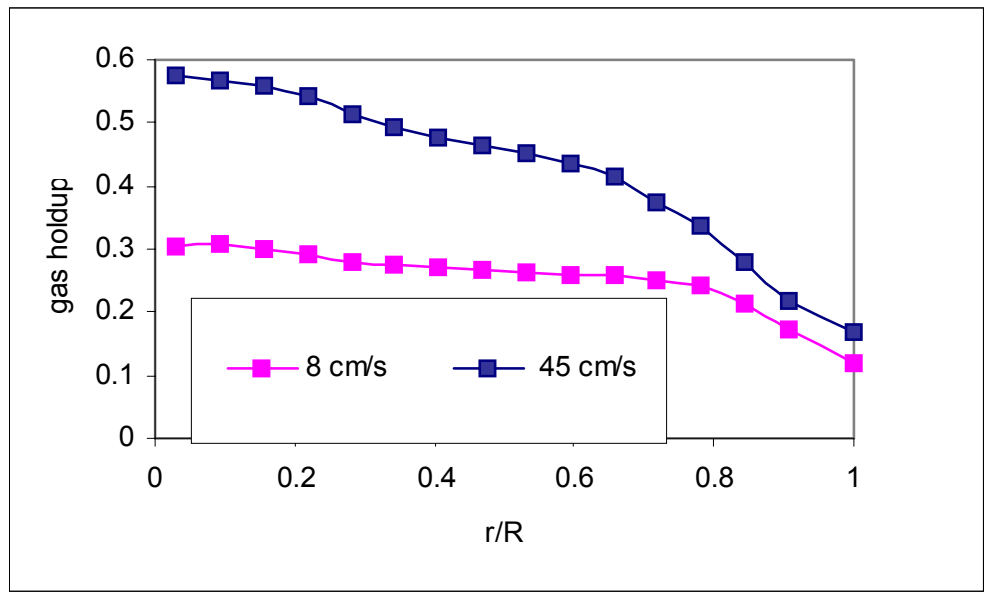

Figure 4.9: Effect of superficial gas velocity on gas holdup profile (air-water-glass beads $150 \mu \mathrm{m}$ ) in 6 " column at $0.1 \mathrm{MPa}$

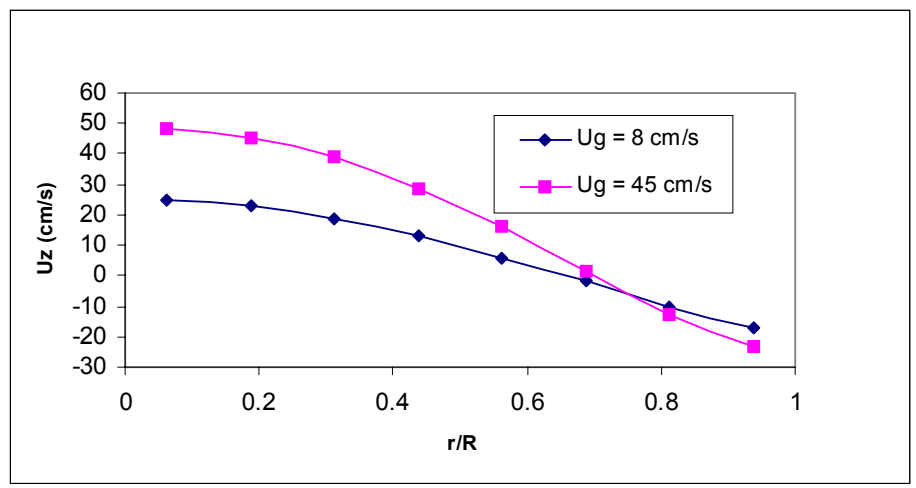

Figure 4.10: Effect of superficial gas velocity on solids axial velocity profile (air-waterglass beads $150 \mu \mathrm{m}$ ) in 6" column with $9.1 \%$ vol. solids loading at $0.1 \mathrm{MPa}$

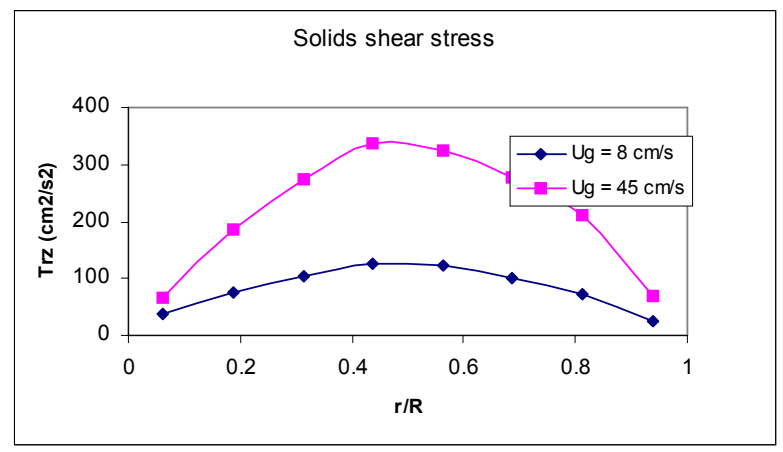

Figure 4.11: Effect of superficial gas velocity on solids shear stress profile (air-waterglass beads $150 \mu \mathrm{m}$ ) in 6" column with $9.1 \%$ vol. solids loading at $0.1 \mathrm{MPa}$ 


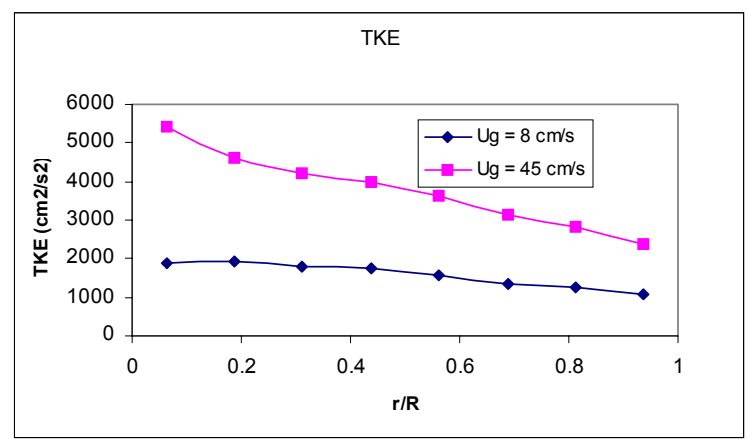

Figure 4.12: Effect of superficial gas velocity on TKE (air-water-glass beads $150 \mu \mathrm{m}$ ) in 6" column with $9.1 \%$ vol. solids loading at $0.1 \mathrm{MPa}$
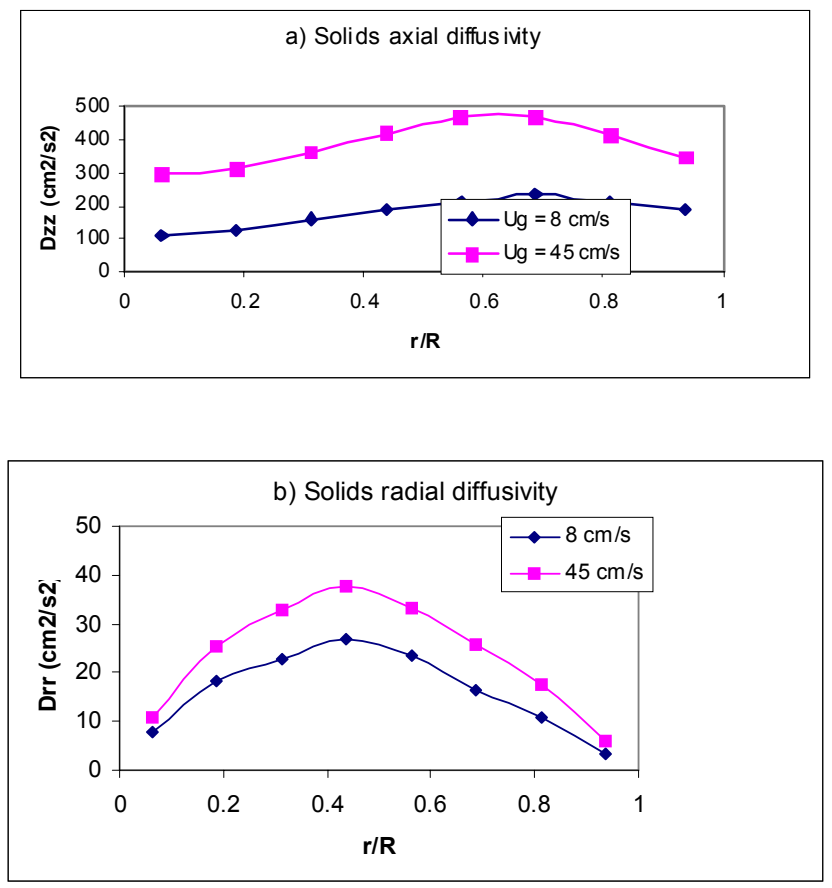

Figure 4.13: Effect of superficial gas velocity on a) solids axial diffusivity, and b) solids radial diffusivity (air-water-glass beads $150 \mu \mathrm{m}$ ) in 6" column with $9.1 \%$ vol. Solids loading at $0.1 \mathrm{MPa}$ 


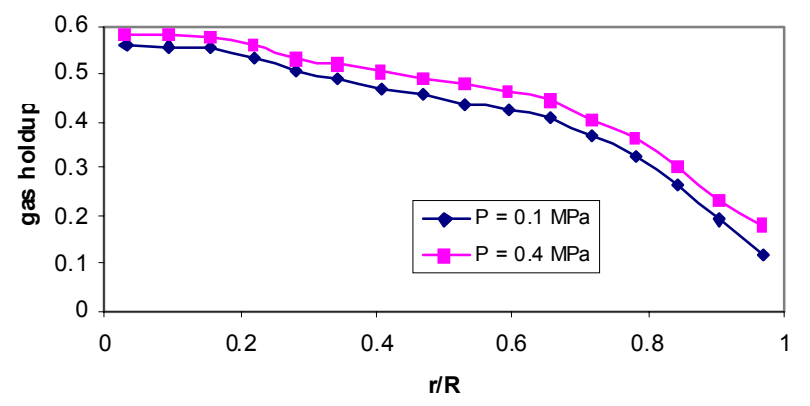

Figure 4.14: Effect of operating pressure on gas holdup radial profile using air-waterglass beads $(150 \mu \mathrm{m})$ in $6 "$ column with $9.1 \%$ vol. solids loading at $45 \mathrm{~cm} / \mathrm{s}$

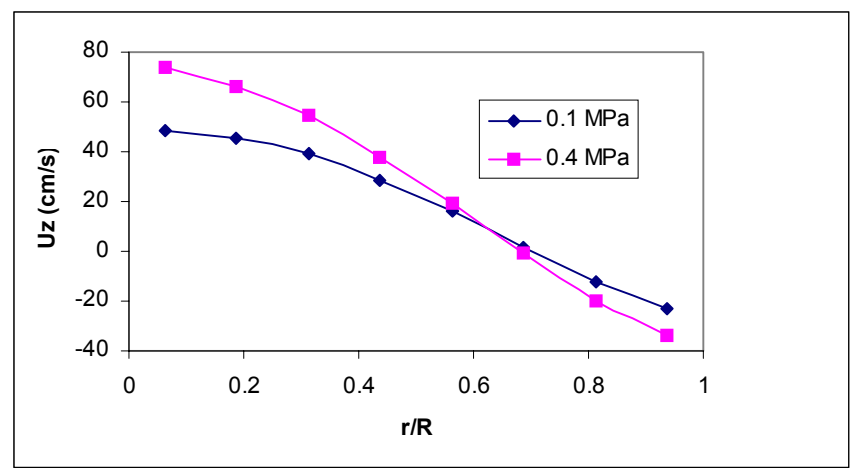

Figure 4.15: Effect of operating pressure on axial velocity profile using air-water-glass beads $(150 \mu \mathrm{m})$ in 6 " column with $9.1 \%$ vol. solids loading at $45 \mathrm{~cm} / \mathrm{s}$

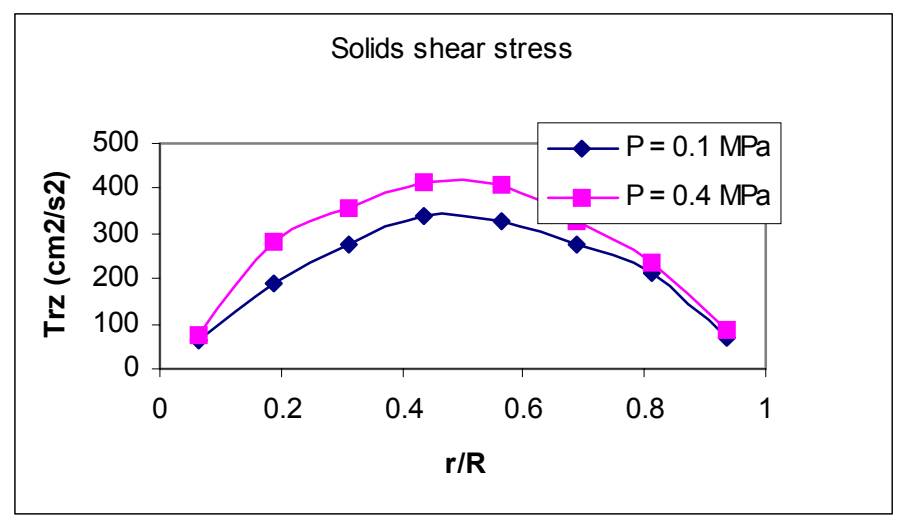

Figure 4.16: Effect of operating pressure on solids shear stress profile using air-waterglass beads $(150 \mu \mathrm{m})$ in $6 "$ column with $9.1 \%$ vol. solids loading at $45 \mathrm{~cm} / \mathrm{s}$ 


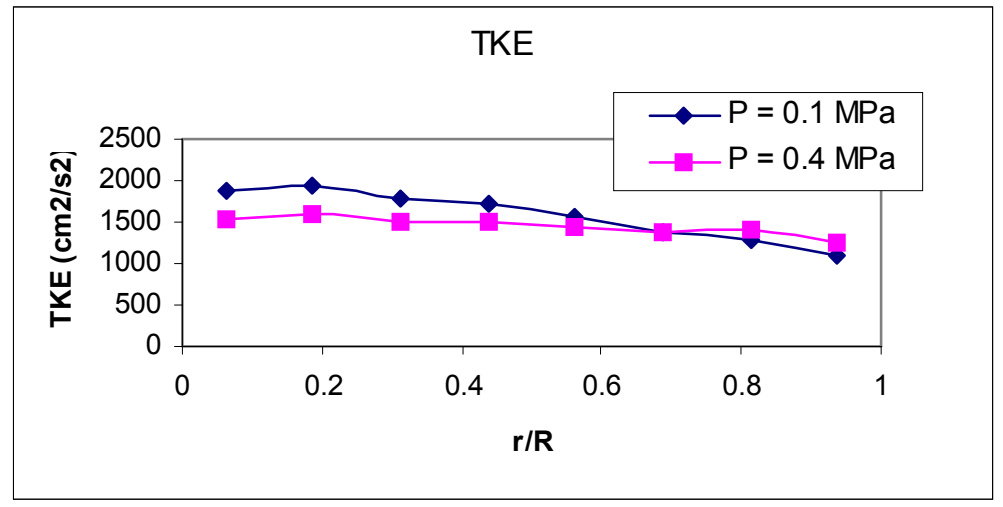

Figure 4.17: Effect of operating pressure on solids TKE using air-water-glass beads (150 $\mu \mathrm{m}$ ) in 6 " column with $9.1 \%$ vol. solids loading at $8 \mathrm{~cm} / \mathrm{s}$
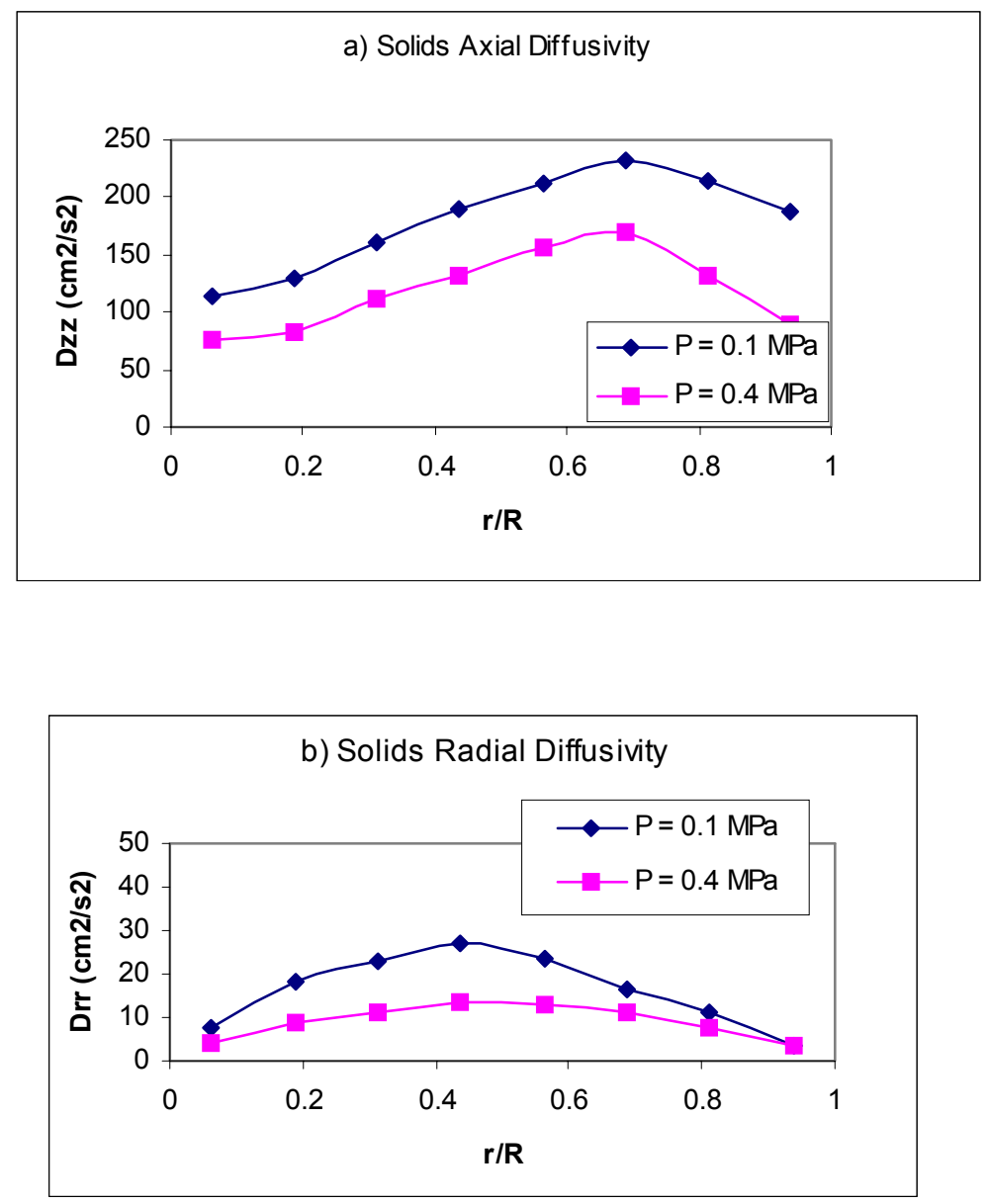

Figure 4.18: Effect of operating pressure on a) solids axial diffusivity profile b) solids radial diffusivity profile using air-water-glass beads $(150 \mu \mathrm{m})$ in 6 " column with $9.1 \%$ vol. solids loading at $8 \mathrm{~cm} / \mathrm{s}$ 


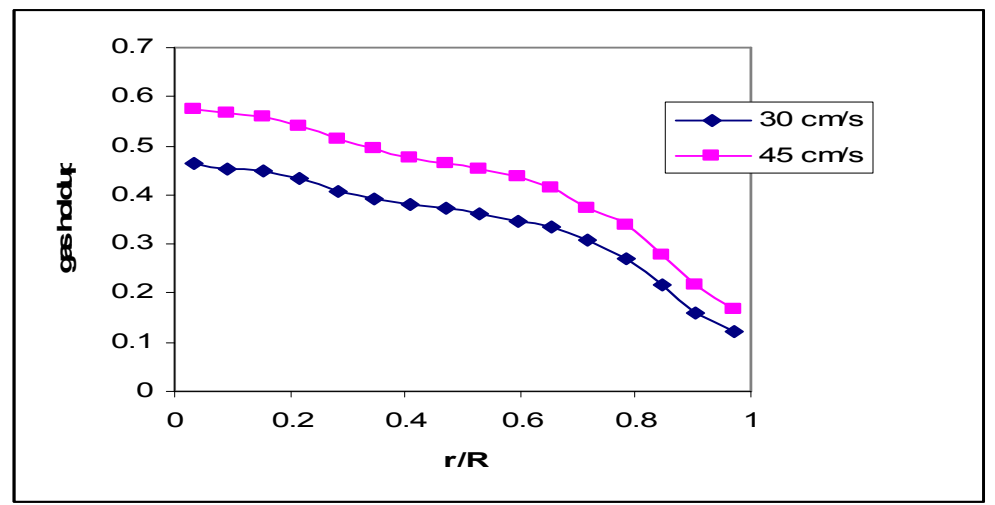

Figure 4.19: Effect of superficial gas velocity on gas holdup profile (air-water) in 6" column at $0.1 \mathrm{MPa}$

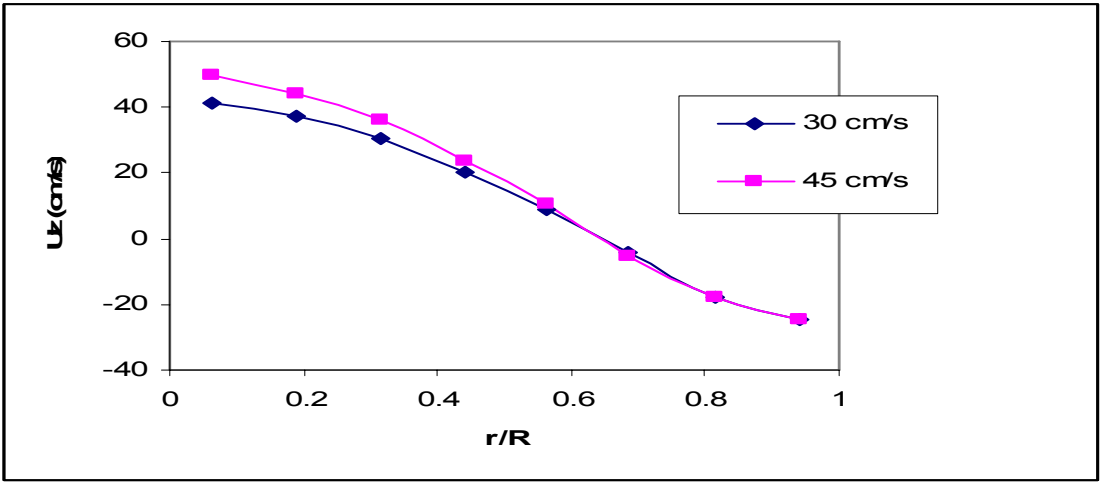

Figure 4.20: Effect of superficial gas velocity on liquid axial velocity profile (air-water) in 6" column at $0.1 \mathrm{MPa}$

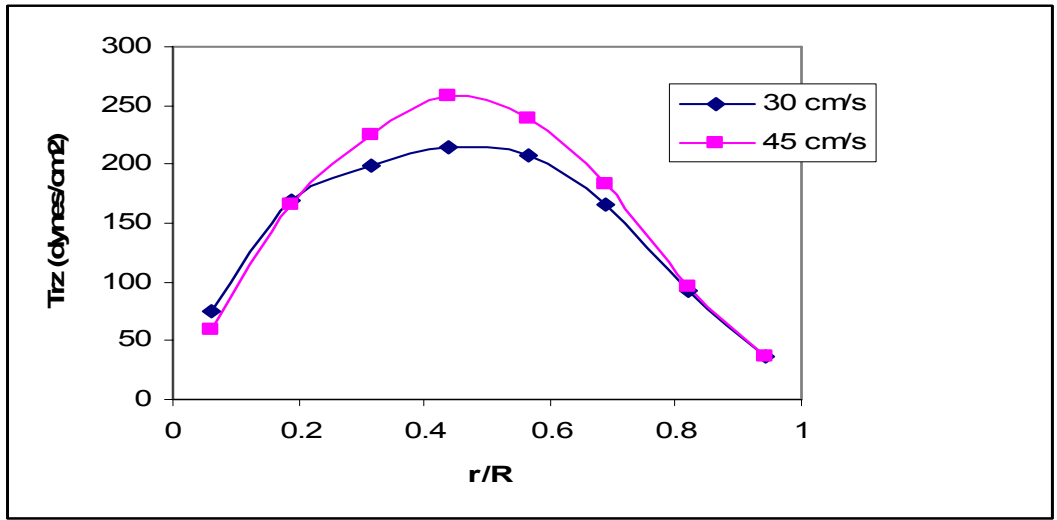

Figure 4.21: Effect of superficial gas velocity on liquid shear stress profile (air-water) in 6 " column at $0.1 \mathrm{MPa}$ 


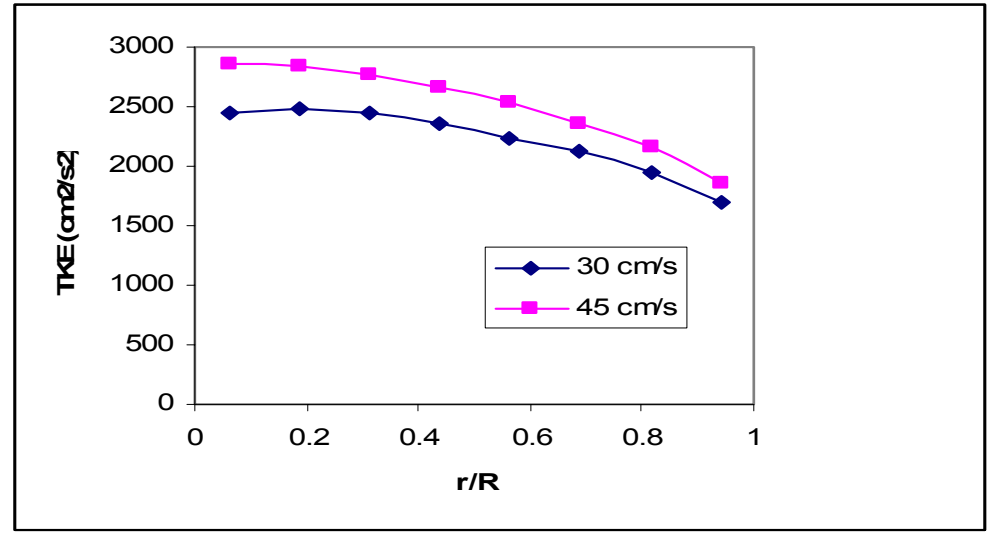

Figure 4.22: Effect of superficial gas velocity on TKE (air-water) in 6" column at 0.1 $\mathrm{MPa}$
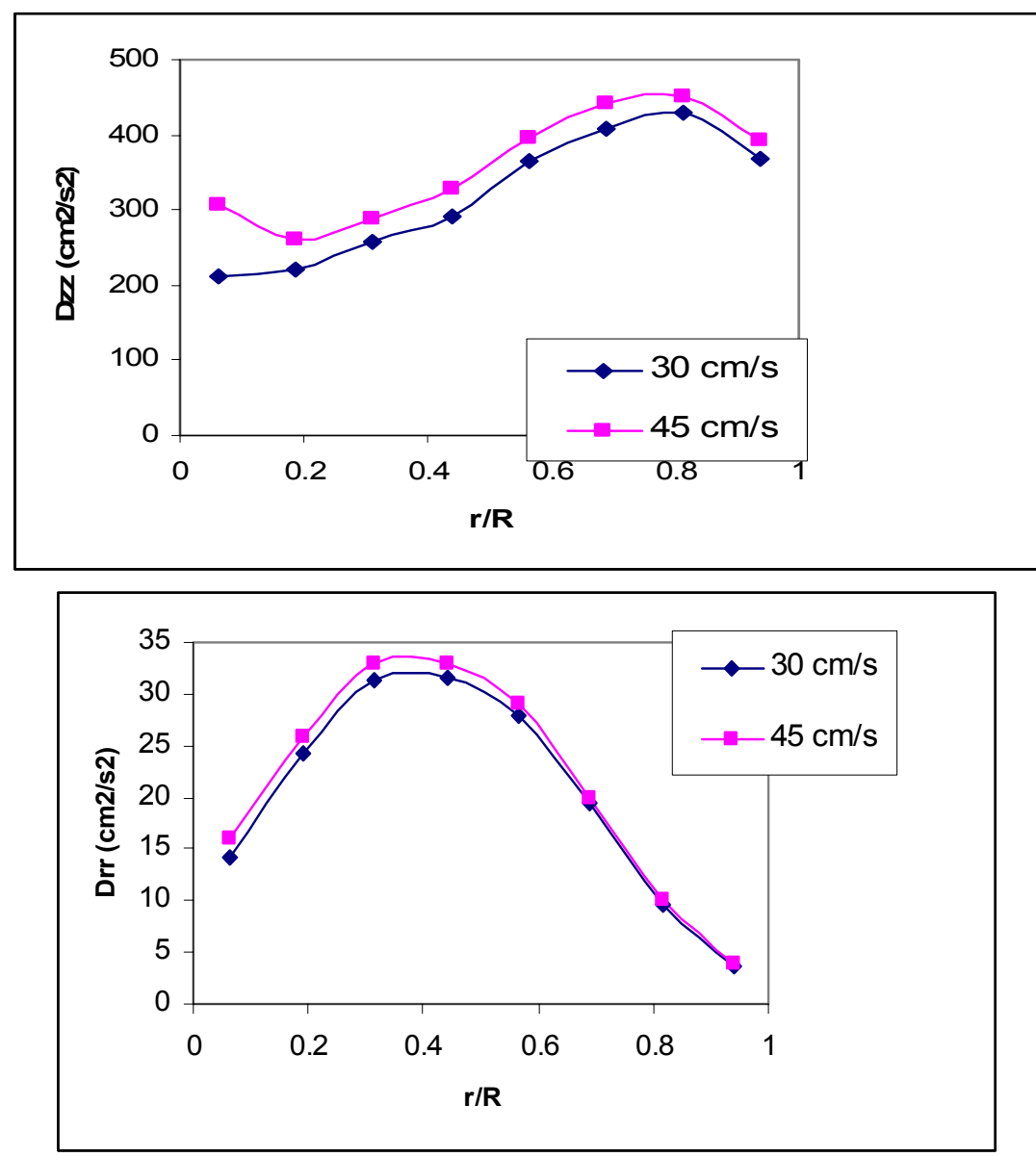

Figure 4.23: Effect of superficial gas velocity on a) liquid axial diffusivity, and b) liquid radial diffusivity (air-water) in 6" column at $0.1 \mathrm{MPa}$ 


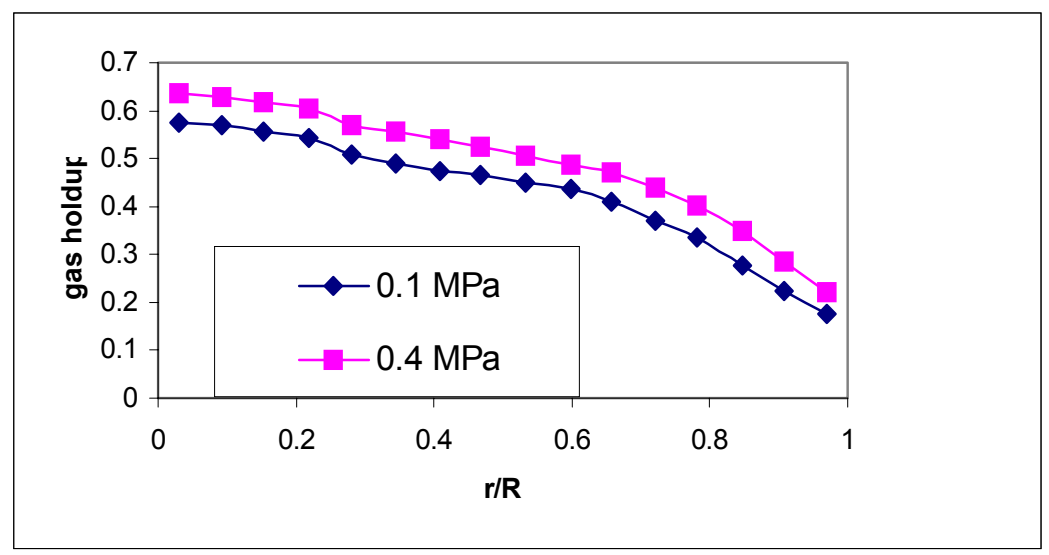

Figure 4.24: Effect of operating pressure on gas holdup radial profile using air-water system in 6" column with at $45 \mathrm{~cm} / \mathrm{s}$

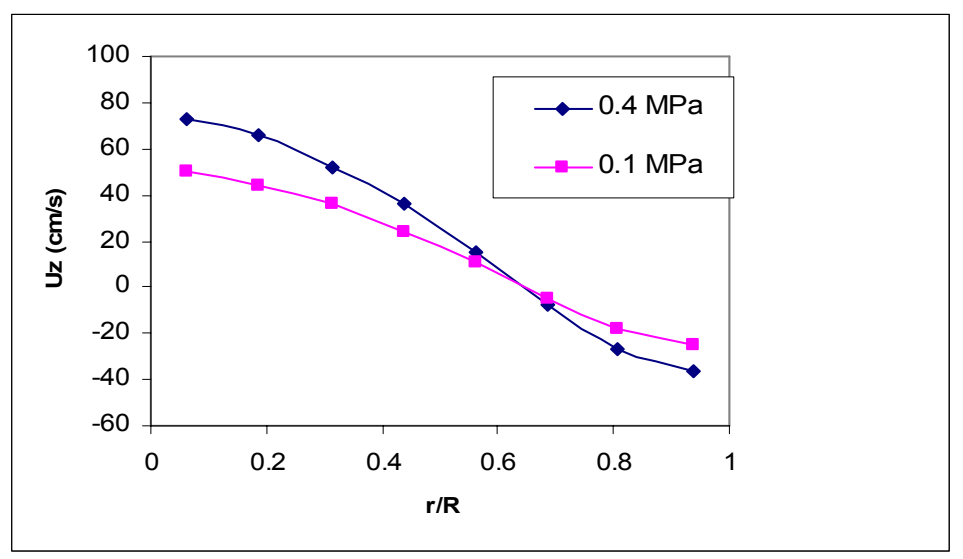

Figure 4.25: Effect of operating pressure on liquid axial velocity profile using air-water sysetm in 6" column at $45 \mathrm{~cm} / \mathrm{s}$

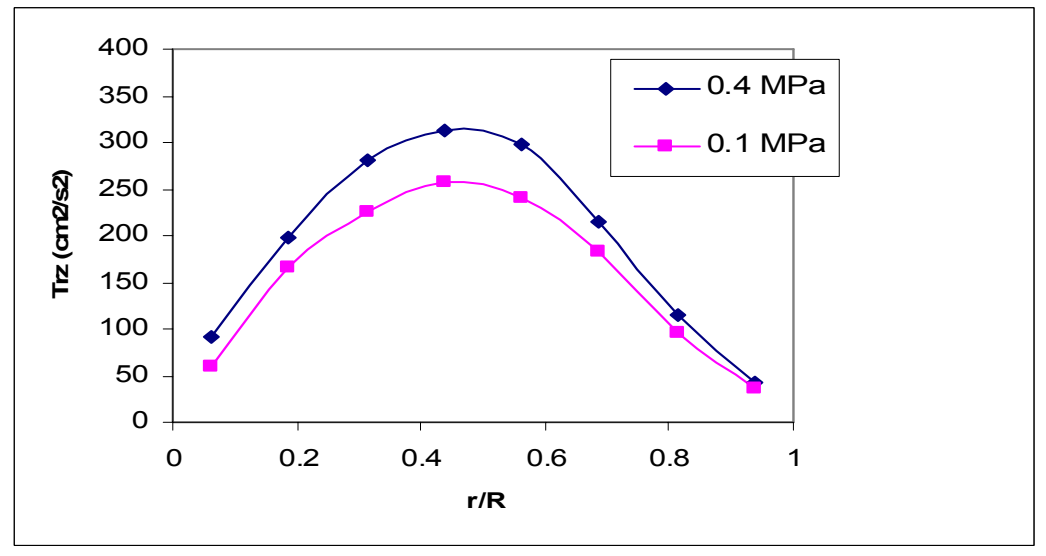

Figure 4.26: Effect of operating pressure on shear stress profile using air-water system in 6" column at $45 \mathrm{~cm} / \mathrm{s}$ 


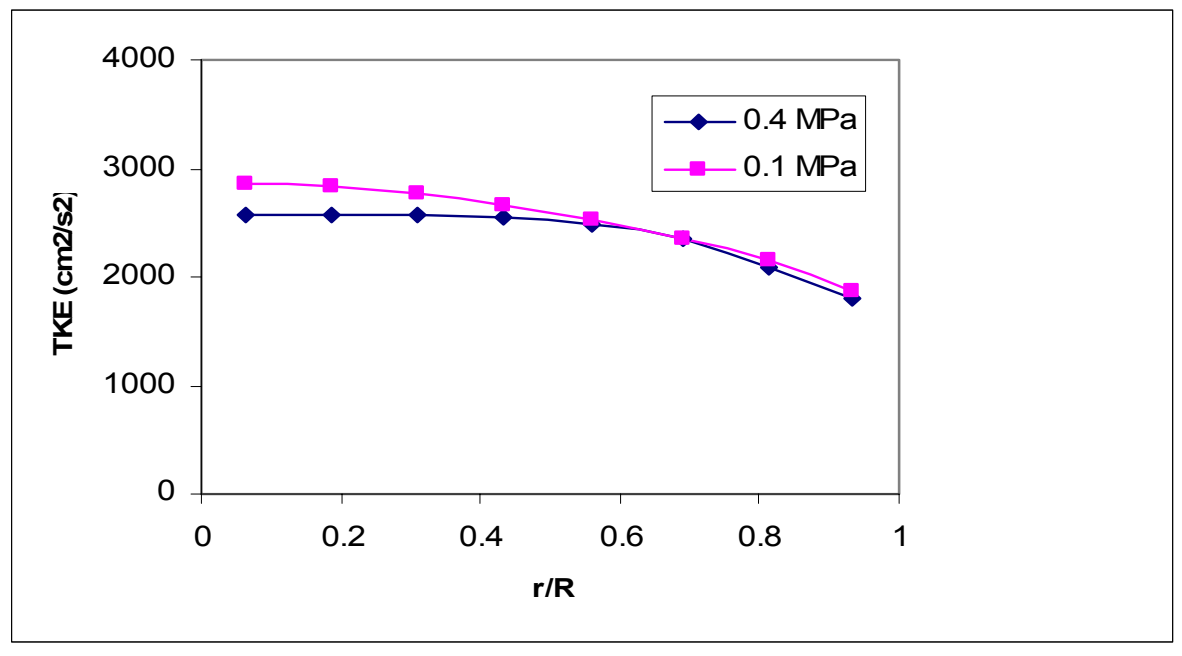

Figure 4.27: Effect of operating pressure on TKE using air-water system in 6" column at $45 \mathrm{~cm} / \mathrm{s}$
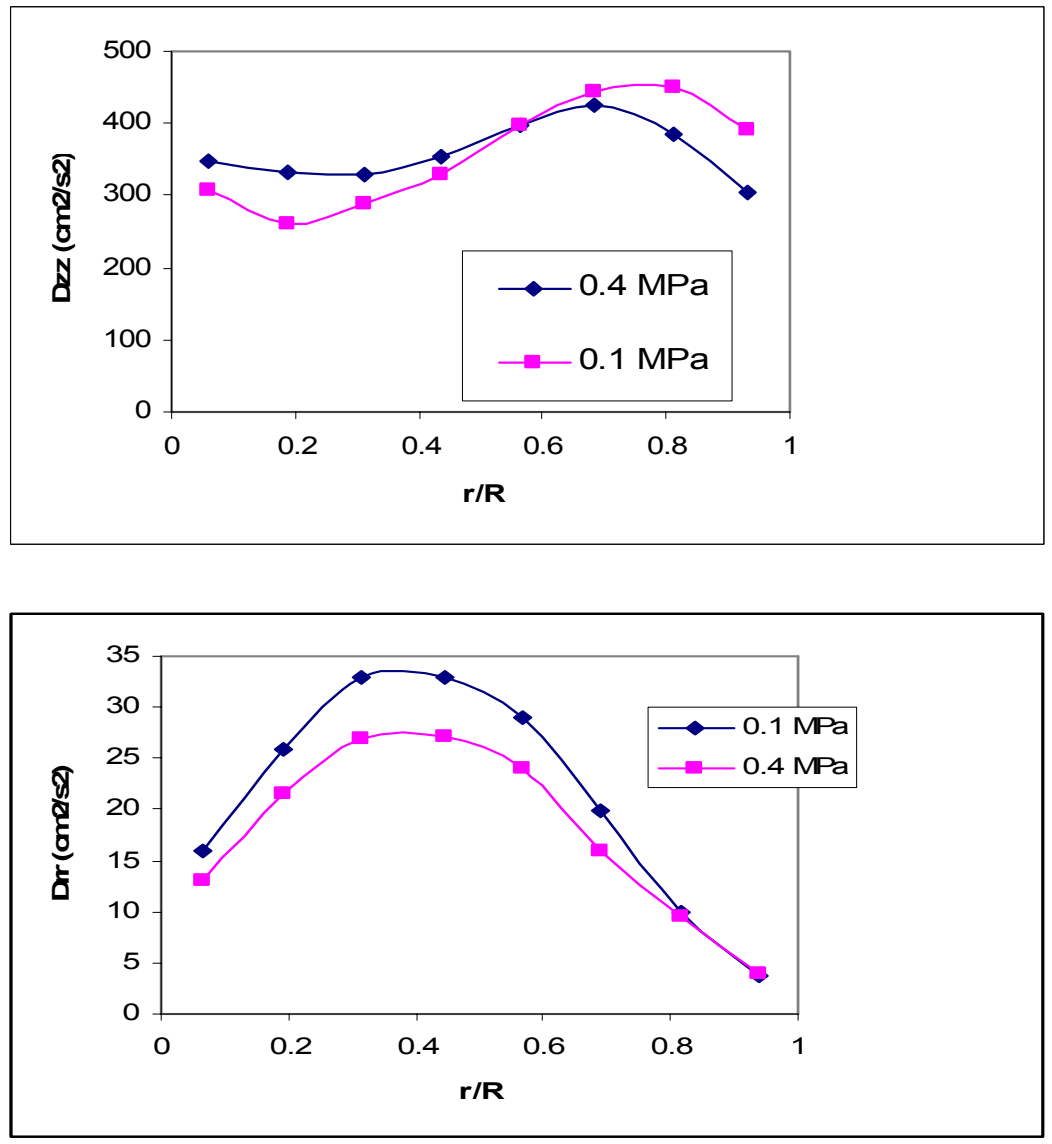

Figure 4.28: Effect of operating pressure on a) axial diffusivity profile b) radial diffusivity profile using air-water system in 6" column at $45 \mathrm{~cm} / \mathrm{s}$ 

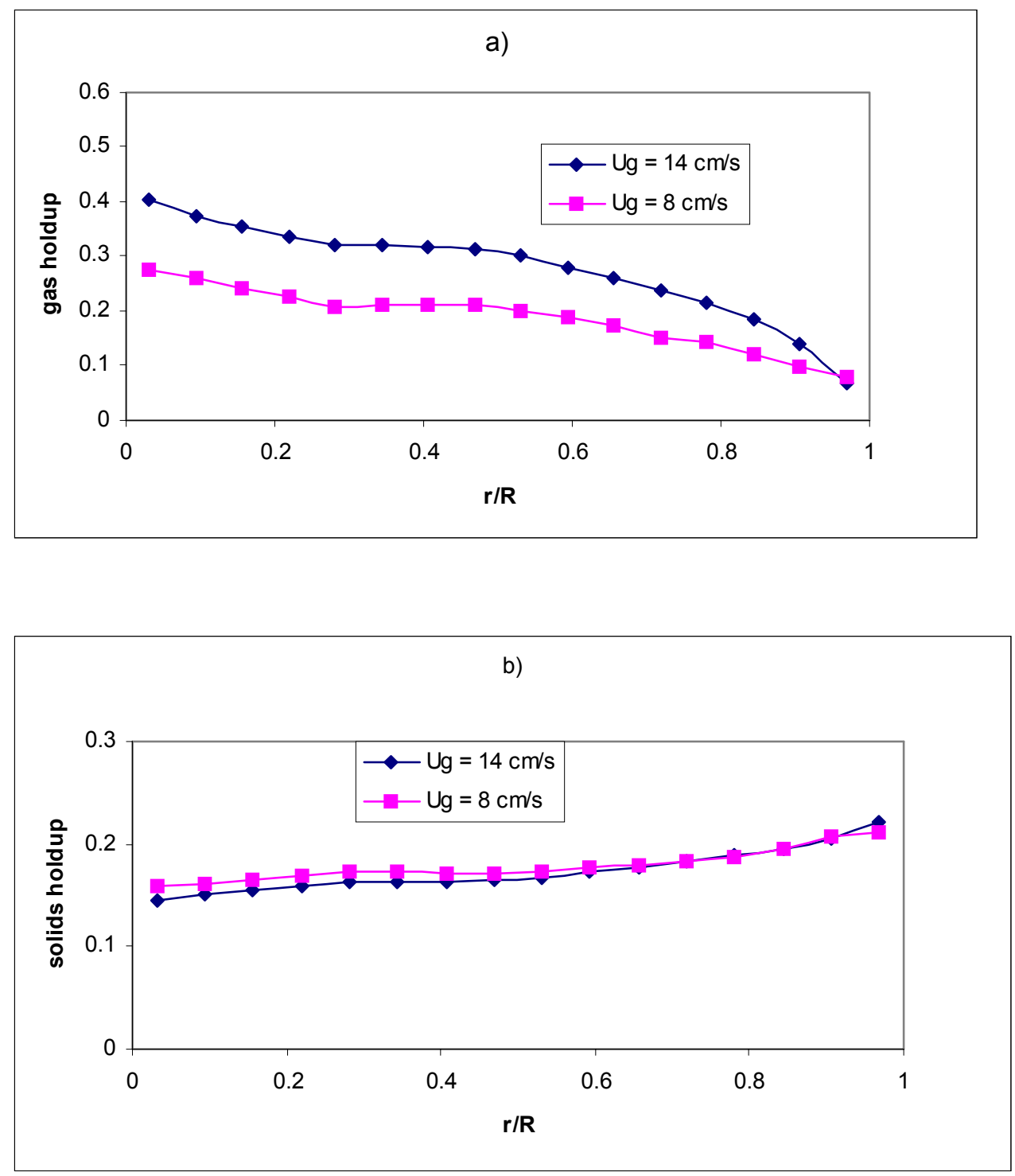

Figure 4.29: Effect of superficial gas velocity on a) gas holdup, and b) solids holdup profile (air - Therminol LT-glass beads) in 6" column with $9.1 \%$ vol. solids loading at $0.1 \mathrm{MPa}$. 

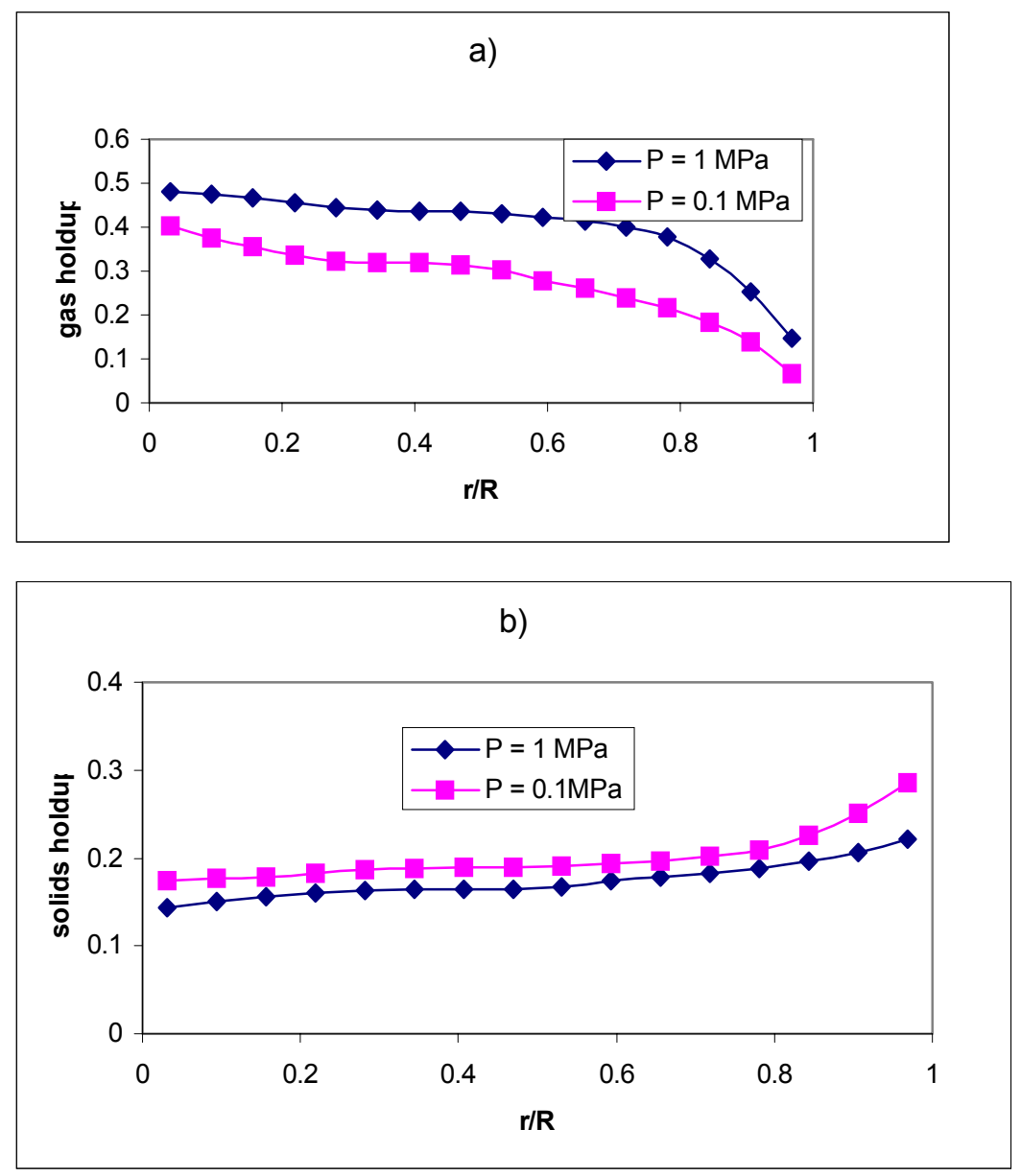

Figure 4.30: Effect of operating pressure on a) gas holdup, and b) solids holdup profile (air- Therminol LT-glass beads) in 6" column with $9.1 \%$ vol. solids loading at $14 \mathrm{~cm} / \mathrm{s}$. 


\section{High-pressure phenomena of bubbles in liquids and liquid-solid suspensions}

Some aspects of bubble dynamics and macroscopic hydrodynamic properties in highpressure bubble columns and three-phase fluidization systems have been reviewed. The details of these discussions are presented in Appendix A as a full manuscript published in Chemical Engineering Science (1999), 54(21), 4681-4709. Experimental results along with discrete-phase simulations of a single bubble rising in liquids and liquid-solid suspensions at high pressures are presented. A mechanistic model is described, which accounts for the initial size of bubble from a single orifice in liquid-solid suspensions. The mechanism for bubble breakup at high pressures is illustrated by considering bubble instability induced by internal gas circulation inside a bubble, and an analytical expression is obtained to quantify the maximum stable bubble size. Experimental examinations on the roles of bubbles of different sizes indicate the importance of large bubbles in dictating the macroscopic hydrodynamics of slurry bubble columns. Further, extensive studies have been made on the key macroscopic hydrodynamic properties, including moving packed bed phenomena, flow regime transition, overall gas holdup, mean bubble size, and bubble size distribution. An empirical correlation is introduced which predicts the gas holdup in slurry bubble columns of different scales. A similarity rule is revealed for the overall hydrodynamics of high-pressure slurry bubble columns, which takes into account the operating conditions, the maximum stable bubble size, and the physical properties of the gas, liquid, and solids. The heat transfer characteristics under high pressures are also investigated. A consecutive film and surface renewal model is used to characterize the heat transfer mechanism. 


\section{Development of a correlation for the prediction of Radial Gas Holdup profiles in Bubble Columns}

A correlation to predict radial gas holdup profiles in bubble columns has been developed based on the data bank obtained at Washington University (WU) via CT, Ohio State University (OSU), and Sandia National Laboratory. The collected experimental data has been fitted into the form proposed by Luo and Svendsen (1991) for gas holdup radial profile. This work utilizes the interdependence between gas holdup radial profile and liquid axial velocity in bubble columns and can be further extended for scale-up and design of such reactors. The details of the developed correlation are described in the full manuscript published in Chemical Engineering Science (2001) (56(3), 1127-1130) attached as Appendix B. 


\section{Development of a correlation for the prediction of axial liquid velocity in Bubble}

Columns

A correlation has been proposed to predict the axial liquid velocity radial profile as a function of the centerline velocity and the parameters that radial gas holdup profiles are correlated to. The data used in such development is collected from the extensive CARPT and PIV experiments performed at WU and OSU as a part of the work funded by various government and industry over the years.

The details of the development are described in the full manuscript published in Chemical Engineering Science (2001) [56(3), 1207-1210] attached in Appendix B. 


\section{Development of an Artificial Neural Network correlation for Prediction of Overall Gas Holdup in Bubble Column Reactors}

In attempt to improve the design and scale-up of bubble columns, a correlation has been proposed to predict overall gas holdup in bubble columns. Around 3500 experimental data points have been collected from literature at a wide range of operating conditions. Based on such an extensive database, a correlation has been developed with the aid of Artificial Neural Network and Dimensional Analysis. It has been shown that the proposed correlation can be useful over a wide range of operating and design conditions. The details of the developed correlation are described in Appendix $\mathrm{C}$ as full manuscript published in Chemical Engineering and Processing (2003), 42(8-9), 599-610. 
9. Prediction of Mass Transfer Coefficient in Bubble Columns operated at High Pressure based on atmospheric pressure data

Several correlations have been proposed for prediction of volumetric mass transfer coefficient in bubble column at atmospheric pressure. Due to difficulties in measuring mass transfer coefficient at high operating pressure, limited database is available at high pressure. By utilizing such a limited database at high pressure, this study develops a procedure for prediction of the volumetric mass transfer coefficient at any pressure based on atmospheric pressure data. The details of this work have been attached as Appendix D. 


\section{Phase Distribution in a High Pressure Slurry Bubble Column via Computed Tomography}

Single source $\gamma$-ray Computed Tomography (CT), which is a technique to measure crosssectional phase holdup distribution in two-phase systems, has been used in the current work. Using a single source $\gamma$-ray CT, a new methodology (CT/Overall gas holdup) has been developed, based on sound assumptions, to calculate phase holdups of all three moving phases. The effects of superficial gas velocity $(8-14 \mathrm{~cm} / \mathrm{s})$ and operating pressure $(0.1-1 \mathrm{MPa})$ on time- and cross-sectionally averaged phase holdups have been investigated in a pilot scale (diameter $0.162 \mathrm{~m})$ SBCR using air-water- $(150 \mu \mathrm{m})$ glass beads and air-Therminol LT- $(150 \mu \mathrm{m})$ glass beads. The detailed analysis of effects of superficial gas velocity, operating pressure, and physical property has been attached as Appendix E, which has been published in the proceeding of $3^{\text {rd }}$ World Congress of Industrial Process Tomography, September 2 - 5, 2003, Banff, Canada. 


\section{Technical Difficulties and Improvements in CARPT}

During the process of implementing CARPT for the first time on high pressure steel column, several serious technical problems and issues arose, which need to be investigated and resolved carefully. Fortunately, all these challenging problems have been successfully identified and resolved to implement CARPT technique at high pressure operation partly as a part of this project and other projects (Industrial Consortium and DF-22-95PC95051) which are executed in parallel with this grant. The details of challenging technical difficulties and their solutions have been attached as Appendix F. 


\section{REFERENCES}

\subsection{Nomenclature}

$\Delta \mathrm{h} \quad$ vertical distance between two tips, $\mathrm{cm}$

$\Delta \mathrm{P} \quad$ Pressure drop

A Absorbance

D Column diameter, $m$

$\mathrm{D}_{\mathrm{rr}} \quad$ solids radial diffusivity, $(\mathrm{cm} / \mathrm{s})^{2}$

$\mathrm{D}_{\mathrm{zz}} \quad$ solids axial diffusivity, $(\mathrm{cm} / \mathrm{s})^{2}$

g gravitational constant, $\mathrm{m} / \mathrm{s}^{2}$

$\mathrm{H} \quad$ Column height, $\mathrm{m}$

$\mathrm{I}, \mathrm{I}_{0} \quad$ intensity of radiation received by detector and emitted by the source

1 bubble chord length, $\mathrm{cm}$

$\mathrm{n}_{\mathrm{s}} \quad$ radioactive particle occurrences

P Pressure inside column, psi

$\mathrm{R}$ radius of column, in

$\mathrm{r} / \mathrm{R} \quad$ Dimensionless radius

$\mathrm{R}_{\mathrm{K}} \quad$ relative volumetric coefficient

$\mathrm{T}$ column temperature, ${ }^{0} \mathrm{C}$

$\mathrm{t}$ time, seconds

$\mathrm{T}_{\mathrm{rz}} \quad$ solids shear stress, $(\mathrm{cm} / \mathrm{s})^{2}$

$\mathrm{U} \quad$ Axial liquid velocity, $\mathrm{cm} / \mathrm{s}$

$\mathrm{u}_{\mathrm{b}} \quad$ bubble rise velocity, $\mathrm{cm} / \mathrm{s}$

Ug Superficial gas velocity, $\mathrm{cm} / \mathrm{s}$

Ur Radial velocity of solids, $\mathrm{cm} / \mathrm{s}$

$\mathrm{Uz}$ Axial velocity of solids, $\mathrm{cm} / \mathrm{s}$

$\mathrm{z} \quad$ column height

\section{Greek letters}

$\overline{v_{S}} \quad$ cross sectional solids loading

$\varepsilon_{G} \quad$ cross-sectional average gas hold up

$\varepsilon_{S} \quad$ solids holdup

$\tau \quad$ time period when bubble is in contact with lower tip of probe, sec

$\rho_{g} \quad$ Gas density, gm/cc

$\rho_{L} \quad$ Liquid density, gm/cc

$\sigma_{L} \quad$ Liquid surface tension, dynes $/ \mathrm{cm}$

$\mu_{L} \quad$ Liquid viscosity, cPs

$\rho \mu \quad$ volumetric attenuation coefficient 


\subsection{References}

Badgujar, M. N., Deimling, A., Morsi, B. I., Shah, Y. T. and Carr, N. L., Solids distribution in a batch bubble column. Chem. Eng. Commun., 48, 127,1986.

Bukur, D.B., Models for Fischer-Tropsch reaction in slurry bubble column reactors, Chem. Eng. Sci., 38(3), 441, 1983.

Chen, J., A. Kemoun, M.H. Al-Dahhan, M.P. Dudukovic, D.J. Lee, L.S. Fan. Comparative hydrodynamics study in a bubble column using CARPT/CT and PIV. Chem. Eng. Sci, 1999.

de Swart, J.W.A, Krishna, R., Sie, S.T., Selection, design and scale up of the FischerTropsch reactor, Stud. Surf. Sci. Catal., 107(Natural Gas Conversion IV), 213, 1997.

de Swart, J.W.A. Scale-up of a Fischer-Tropsch slurry reactor. Ph.D. Thesis, University of Amsterdam, The Netherlands. 1996.

Deckwer, W.-D., Serpemen, Y, Ralek, M. and Schmidt B., Modeling the Fischer-Tropsch Synthesis in the slurry phase, Ind. Eng. Chem. Process Des. Dev., 21, 231, 1982.

Degaleesan, S., Turbulence and liquid mixing in bubble columns, D.Sc. Thesis, Washington University, St. Louis, MO, 1997.

Devanathan, N. Investigation of liquid hydrodynamics in bubble columns via computer automated radioactive particle tracking (CARPT). D.Sc Thesis, Washington University, St. Louis, 1991.

Dry, M.E., Advances in Fisher-Tropsch chemistry, Ind. Eng. Chem. Prod. Res. Dev., 15, 282, 1976.

Fan, L.-S. and K. Tsuchiya. Bubble Wake Dynamics in Liquids and Liquid-Solid Suspensions. Butterworth-Heinemann, Stoneham, MA, 1990.

Fan, L.-S., G.Q. Yang, D.J. Lee, K. Tsuchiya and X. Luo. Some aspects of high-pressure phenomena of bubbles in liquids and liquid-solid suspensions. Chem. Eng. Sci., 54, 4681, 1999.

Fan, L.-S., Gas-liquid-solid fluidization engineering, Butterworths Series in Chemical Engineering, Boston, 1989.

Gormley, R.J., Zarochak, M.F., Deffenbaugh, P.W. and Rao, K.R.P.M., Effect of initial wax medium on the Fishcer-Tropsch slurry reaction, Applied Catalysis, 161, 263-279, 1997.

Grevskott, S., B.H. Sannaes, M.P. Dudukovic, K.W. Hiarbo, and H.F. Svendsen. Liquid circulation, bubble size distribution and solids movements in two- and three-phase bubble 
columns. Chem. Eng. Sci., 51, 1703-1713, 1996.

Hammer, H, H. Schrag, K. Hektor, K. Schonau, W. Kuster, A. Soemarno, U. Sahabi, and W. Napp, New subfunctions on hydrodynamics, heat and mass transfer for gas/liquid and gas/liquids/solid chemical and biochemical reactors, Front. Chem. Reac. Eng., 464, 1984.

Hills, J.H. Radial non-uniformity of velocity and voidage in a bubble column. Trans. Inst. Chem. Engrs., 52, 1-9, 1974.

Hu, T., Yu, B. and Wang, Y., Holdup and models of three phase fluidized bed. Fluidization $V, 353,1986$.

Huff, G.A.Jr. and Satterfield, C.N., Intrinsic kinetics of the Fischer-Tropsch synthesis on a reduced fused-magnetite catalyst, Ind. Eng. Chem. Process Des. Dev., 23(4), 696, 1984.

Idogawa, K., Ikeda, K., Fukuda, T., and Morooka, S., 'Effect of Gas and Liquid Properties on the Behavior of Bubbles in a Bubble Column under High Pressure,' Kag. Kog. Ronb., 11, 432, 1985.

Idogawa, K., Ikeda, K., Fukuda, T., and Morooka, S., 'Effect of Gas and Liquid Properties on the Behavior of Bubbles in a Column under High Pressure,' Int. Chem. Eng., 27, 93-99, 1987.

Jean, R.-H. and L.-S. Fan. Rise velocity and gas-liquid mass transfer of a single large bubble in liquids and liquid-solid fluidized beds. Chem. Eng. Sci., 45, 1057, 1990.

Joshi, J.B. and M.M. Sharma. A circulation cell model for bubble columns. Trans. Inst. Chem. Engrs, 57, 244-251, 1979.

Kato, Y., Nishiwaki, A., Fukuda, T. and Tanaka, S., The behavior of suspended solid particles and liquid in bubble columns, J. Chem. Eng. Japan, 5, 112, 1972.

Kemoun, A., Ong, B. C., Gupta, P., Al-Dahhan, M. H., and Dudukovic', M. P., 'Gas Holdup in Bubble Columns at Elevated Pressure via Computed Tomography,' International J. of Multiphase Flows, 27(2001) 929-946.

Kondukov, N. B., Kornilaev, A. N., Skachko, I. M., Akhromenkov, A. A. and Kurglov, A. S., An invetsigation of the parameters of moving particles in a fluidized bed by a radioisotopic method. Int. Chem. Eng., 4, 43, 1964.

Krishna, R., and Ellenberger, J., 'Gas Holdup in Bubble Column Reactors Operating in the Churn-Turbulent Flow Regime,' AIChE J., 42, 9, 2627-2634, 1996.

Kumar, S.B., Computed tomography measurements of void fraction and modeling of the flow in bubble columns, Ph.D. Thesis, Florida Atlantic University, Boca Raton, 1994. 
Leib, T.M., Mills, P.L., Lerou, J.J. and Turner, J.R., Evaluation of Neural Networks for simulation of three-phase bubble column reactors, Trans. Inst. Chem. Eng. (Part A), 73, 690, 1995.

Limtrakul, S., Hydrodynamics of liquid fluidized beds and gas-liquid fluidized beds. D.Sc. Thesis, Washington University, St. Louis, MO,1996.

Lin, T.-J., Tsuchiya, K. and Fan, L.-S., Bubble flow characteristics in bubble columns at elevated pressure and temperature, AIChE J., 44, 545, 1998.

Lin, T.-J., Tsuchiya, K., and Fan, L.-S., "On the measurements of regime transition in high-pressure bubble columns", Can. J. of Chem. Eng., 77, 370374, (1999).

Luo X., D.J. Lee, R. Lau, G.Yang and L.S. Fan, Maximum stable bubble size and gas hold up in high pressure slurry bubble columns, AICHE J., 45, 665-680, 1999.

Luo, X., G. Q. Yang, D. J. Lee, and L.-S. Fan. Single bubble formation in high pressure liquid-solid suspensions. Powder Technology, 100, 103, 1998.

Luo, X., Lee, D. J., Lau, R., Yang, G. and Fan, L.-S., Maximum stable bubble size and gas holdup in high pressure slurry bubble columns. AIChE J., 45, 665, (1999).

Marano, J.J. and Holder, G.D., Prediction of bulk properties of Fischer-Tropsch derived liquid, Ind. Eng. Chem. Res., 36(6), 2409-2420, 1997.

Maretto, C. and Krishna, R., Modeling of a bubble column slurry reactor for FischerTropsch synthesis. Catalysis Today. 52, 279-289, 1999.

Matsumoto, T., Hidaka, N., Gushi, H. and Morooka, S., Axial segregation of multicomponent solid particles suspended in bubble columns. Ind. Eng. Chem. Res., 31, 1562,1992 .

Matsumoto, T., Hidaka, N., Gushi, H. and Morooka,S., Axial segregation of multicomponent solid particles suspended in bubble columns. Ind. Eng. Chem. Res., 31, 1562,1992 .

Mendelson, H. D. The motion of an air bubble rising in water. AIChE J., 13, 250, 1967.

Mills, P.L., Turner, J.R., Ramachandran, P.A. and Dudukovic, M.P., The FischerTropsch synthesis in slurry bubble column reactors: analysis of reactor performance using the axial dispersion model, Topics Chem. Eng., 8(Three Phase Sparged Reactors), 339, 1996.

Moslemian, D., Devanathan, N. and Dudukovic, M. P., Radioactive particle tracking technique for investigation of phase recirculation and turbulence in multiphase systems. Rev. Sci. Instrum., 63, 4361,1992.

Murray, P and Fan, L.-S., Axial solid distribution in slurry bubble columns, Ind. Eng. Chem. Res., 28, 1697, 1989. 
Ong, B.-C., N. Rados, P. Gupta, Y. Wu, M.H. Al-Dahhan, M.P. Dudukovic, R. Lau, L.S. Fan. Hydrodynamic of slurry bubble column. Internal report, Chemical Reaction Engineering Laboratory (CREL), Washington University, St. Louis, 2000.

Oukachi R., Singelton, A.H. and Goodwin, J.G., Comparison of patented Co F-T catalysts using fixed-bed and slurry bubble column recators, Appl. Catal., 186(1-2), 129, 1999.

Patel, S.A., Daly, J.G. and Bukur, D.B., Bubble-Size Distribution in Fischer-TropschDerived Waxes in a Bubble Column, AIChE J., 36(1), 93, 1990.

Patel, S.A., Daly, J.G. and Bukur, D.B., Bubble-Size Distribution in Fischer-TropschDerived Waxes in a Bubble Column, AIChE J., 36(1), 93, 1990.

Prakash, A., On the effect of syngas composition and water-gas-shift reaction rate on FT synthesis over iron based catalyst in a slurry reactor, Chem. Eng. Commun., 128, 143, 1993.

Rados, N. Slurry bubble column hydrodynamics, D.Sc. Proposal, Washington University, St. Louis, 1999.

Rados, N., Slurry bubble column hydrodynamics, D.Sc. Thesis, Washington University, St. Louis, 2003.

Rao, V.U.S., Stiegel, G.J., Cinquegrane, G.J. and Srivastava, R.D., Iron-based catalyst for slurry-phase Fischer-Tropsch process: Technology review, Fuel Process. Technol., 30 (1), 83, 1992.

Rice, R.G., N.W. Geary. Prediction of liquid circulation in viscous bubble columns. AIChE J., 36, 1339-1348, 1990.

Sannaes, B.H., M.P. Dudukovic, and H. Svendsen. Experimental and numerical investigation of solids dynamics in slurry bubble columns, in Hamid Amstoopour, Editor, Fluidization and Fluid-Particle Systems, preprints, 159-163, Particle Forum of AIChE, 1995.

Shah, Y. T., B. G. Kelkar, S. P. Godbole, and W.-D. Deckwer. Design parameters estimations for bubble column reactors. AIChE J., 28, 353, 1982.

Soong, Y., Harke, F.W., Gamwo, I.K., Schehl, R.R. and Zarochak, M.F., Hydrodynamic study in a slurry-bubble column reactor, Catalysis Today, 35, 427-434, 1997.

Soong, Y., Harke, F.W., Gamwo, I.K., Schehl, R.R. and Zarochak, M.F., Hydrodynamic study in a slurry-bubble column reactor, Catalysis Today, 35, 427-434, 1997.

Srivastava, R.D., Rao, V.U.S. Cinquegrane, G. and Stiegel, G.J., Catalysts for FischerTropsch, Hydrocarbon Porcess., Int. Ed., 69(2), 59, 1990. 
Stern, D., Bell, A.T. and Heinemann, H., A theoretical model for the performance of bubble-column recators used for Fischer-Tropsch synthesis, Chem. Eng. Sci., 40(9), $1665,1985$.

Storch, H.H., Golumbic, N. and Anderson, R.B., The Fischer-Tropsch and related synthesis, John Wiley \& Sons, Inc., New York, 1951.

Tomiyama, A., I. Kataoka, and T. Sakaguchi. Drag coefficients of bubbles (1st report, drag coefficients of a single bubble in a stagnant liquid). Nippon Kikai Gakkai Ronbunshu B Hen, 61(587), 2357, 1995.

Turner, J.R. and Mills, P.L., Comparison of axial dispersion and mixing cell models for design and simulation of Fischer-Tropsch slurry bubble column reactors, Chem. Eng. Sci., 45(8), 2317, 1990.

van der Laan, G.P., Beenackers, A.A.C.M. and Krishna, R., Multicomponent reaction engineering model for Fe-catalyzed Fischer-Tropsch synthesis in commercial scale slurry bubble column reactors, Chem. Eng. Sci., 54, 5013, 1999.

Wasan, D. T. and M. S. Ahluwalia., Consecutive film and surface renewal mechanism for heat and mass transfer from a wall. Chem. Eng. Sci., 24, 1535, 1969.

Wilkinson, P.M., A.P. Apek, and L.L. van Dierendonck, Design parameters estimation for scale-up of high-pressure bubble columns, AICHE J.,38(4), 544-554, 1992.

Zhang, J., L.-S. Fan, C. Zhu, R. Pfeffer, and D. Qi. Dynamic behavior of collinear collision of elastic spheres in viscous fluids, Advanced Technologies for Particle Processing, Vol. II, 44, Particle Technology Forum, AIChE; Proceedings of PTF Topical Conference at AIChE Annual Meeting, Nov. 15-20, Miami Beach, FL; Powder Technology, in press, 1998a.

Zhang, J., Y. Li, and L.-S. Fan. Numerical simulation of gas-liquid-solid fluidization systems using a combined CFD-DPM-VOF method: single bubble rise behavior, Advanced Technologies for Particle Processing, Vol. II, 509, Particle Technology Forum, AIChE; Proceedings of PTF Topical Conference at AIChE Annual Meeting, Nov. 15-20, Miami Beach, FL, 1998b. 


\section{APPENDIX A}

\section{Some Aspects of High-Pressure Phenomena of Bubbles in Liquids and Liquid-Solid Suspensions}




\title{
Some aspects of high-pressure phenomena of bubbles in liquids and liquid-solid suspensions
}

\author{
L.-S. Fan, G. Q. Yang, D. J. Lee, K. Tsuchiya, and X. Luo \\ Department of Chemical Engineering \\ The Ohio State University \\ Columbus, Ohio 43210 \\ U.S.A.
}

\begin{abstract}
Some aspects of bubble dynamics and macroscopic hydrodynamic properties in high-pressure bubble columns and three-phase fluidization systems are discussed. Experimental results along with discrete-phase simulations of a single bubble rising in liquids and liquid-solid suspensions at high pressures are presented. A mechanistic model is described, which accounts for the initial size of bubble from a single orifice in liquid-solid suspensions. The mechanism for bubble breakup at high pressures is illustrated by considering bubble instability induced by internal gas circulation inside a bubble, and an analytical expression is obtained to quantify the maximum stable bubble size. Experimental examinations on the roles of bubbles of different sizes indicate the importance of large bubbles in dictating the macroscopic hydrodynamics of slurry bubble columns. Further, extensive studies are made of the key macroscopic hydrodynamic properties, including moving packed bed phenomena, flow regime transition, overall gas holdup, mean bubble size, and bubble size distribution. An empirical correlation is introduced which predicts the gas holdup in slurry bubble columns of different scales. A similarity rule is revealed for the overall hydrodynamics of highpressure slurry bubble columns, which takes into account the operating conditions, the maximum stable bubble size, and the physical properties of the gas, liquid, and solids. The heat transfer characteristics under high pressures are also investigated. A consecutive film and surface renewal model is used to characterize the heat transfer mechanism.
\end{abstract}

Keywords - bubble breakup, bubble dynamics, bubble formation, bubble rise velocity, highpressure three-phase fluidized bed, maximum stable bubble size, slurry bubble column 


\section{INTRODUCTION}

Gas-liquid bubble columns and three-phase fluidization systems are widely used in industry, particularly chemical and petrochemical industries. Three-phase fluidization describes a gas-liquidsolid flow system in which particles are in motion induced by gas and/or liquid phases. Fundamental studies of transport phenomena in bubble columns or three-phase fluidization systems have been extensive over the past decades, and comprehensive reviews are available (Shah et al., 1982; Fan 1989; Deckwer, 1992; Saxena and Chen, 1994). Most studies were conducted under ambient conditions, and relatively little is known regarding high-pressure systems with relevance to industrial processes. Many industrial processes of considerable commercial interest are conducted under high pressures. Examples are: methanol synthesis (at $P=5.5 \mathrm{MPa}$ and $T=0^{\circ} \mathrm{C}$ ), resid hydrotreating (at $P$ $=5.5$ to $21 \mathrm{MPa}$ and $T=300$ to $425^{\circ} \mathrm{C}$ ), Fischer-Tropsch synthesis (at $P=1.5$ to $5.0 \mathrm{MPa}$ and $T=$ $250^{\circ} \mathrm{C}$ ), and benzene hydrogenation (at $P=5.0 \mathrm{MPa}$ and $T=180^{\circ} \mathrm{C}$ ) (Fox, 1990; Jager and Espinoza, 1995; Saxena, 1995; Mill et al., 1996; Peng et al., 1998).

This paper is intended to address the recent advances in transport phenomena of high-pressure bubble columns and three-phase fluidization systems. Selected areas of research centered around the work recently completed by the research group of the senior author at the Ohio State University are discussed together with some relevant works reported in the literature. Experimental results obtained at the Ohio State University are from a high-pressure/high-temperature system of 2- and 4-inch ID columns. The system can be operated at pressures up to $21 \mathrm{MPa}$ and temperatures up to $180^{\circ} \mathrm{C}$. Three pairs of windows installed on the column wall allow direct flow visualization to be carried out. Various types of intrusive high-pressure and high-temperature probes, such as the optical fiber probe, and microfoil heat transfer probe, are developed and used to obtain the bubble characteristics and transport properties of the phases. Furthermore, various techniques via visualization yield in-situ physical properties of the fluids, e.g., the emerging-bubble technique for the surface tension measurement, the hydrostatic weighing method for the density measurement, and the falling-ball technique for the viscosity measurement (Lin and Fan, 1997; Lin et al., 1998).

The selected high-pressure areas discussed in this paper include the bubble dynamics, covering single bubble rise velocity, bubble formation, and bubble breakup, and the macroscopic hydrodynamic properties, covering moving packed bed phenomena, flow regime transition, overall gas holdup, bubble size, and bubble size distribution, in bubble columns and three-phase fluidized beds. Bubbles rising in liquids and liquid-solid suspensions are examined experimentally as well as numerically. A mechanistic model is described on the bubble formation process from a single orifice 
in liquid-solid suspensions. The bubble breakup at high pressures is illustrated by considering bubble instability induced by the internal gas circulation inside a bubble, and further, an analytical expression is obtained to quantify the maximum stable bubble size. A correlation is provided to obtain the gas holdup in bubble and slurry bubble columns over a wide range of flow conditions. A similarity rule is revealed for the overall hydrodynamics of high-pressure slurry bubble columns, which takes into account the operating conditions, the maximum stable bubble size, and the physical properties of the gas, liquid, and solids. The heat transfer characteristics are also discussed.

\section{BUBBLE DYNAMICS}

\subsection{Single bubble rise velocity}

The characteristics of a rising bubble can be described in terms of the rise velocity, shape and motion of the bubble. These rise characteristics are closely associated with the flow and physical properties (mainly viscosity and presence/absence of solid particles) of the surrounding medium as well as the interfacial properties (i.e. presence/absence of surfactant) of the bubble surface. The bubble rise velocity, $u_{b}$, is the single most critical parameter in characterizing the hydrodynamics and transport phenomena of bubbles in liquids and liquid-solid suspensions (Fan and Tsuchiya, 1990). The rise velocity of a single gas bubble inherently depends on its size: for small bubbles, the rise velocity strongly depends on liquid properties such as surface tension and viscosity; for large bubbles, the rise velocity is insensitive to liquid properties (Fan, 1989). Under limited conditions, the rise velocities of single bubbles in liquid-solid suspensions were found to be similar to those in highly viscous liquids (Massimilla et al., 1961; Darton and Harrison, 1974). Liquid-solid suspensions can thus be characterized as Newtonian homogeneous media, but they often exhibit non-Newtonian or heterogeneous behavior (Tsuchiya et al., 1997). Studies in the literature on the bubble rise velocity in liquid-solid suspensions were mainly conducted in water-suspended/fluidized systems and mostly under ambient conditions. Differences in fluidizing media, pressure, and temperature may lead to different bubble rise characteristics.

This section focuses on the bubble rise characteristics in liquids and liquid-solid fluidized beds under frequently encountered industrial conditions, i.e. elevated pressure and temperature, and with a non-water based liquid medium. In liquid-solid suspensions under these conditions, the bubble rise velocity is discussed in light of both the apparent homogeneous (or effective) properties of the suspension and the recently evolved numerical prediction based on a computational model for gas-liquid-solid fluidization systems. 


\subsubsection{In liquids}

Krishna et al. (1994) studied the pressure effect on the bubble rise velocity and found that the single bubble rise velocity does not depend on the gas density over the range of 0.1 to $30 \mathrm{~kg} / \mathrm{m}^{3}$. The conclusion is limited to a narrow range of pressures. Lin et al. (1998) measured the rise velocity of single bubbles of known sizes in Paratherm NF heat transfer fluid at various pressures ranging from 0.1 to $19.4 \mathrm{MPa}$ for three temperatures: 27,47 , and $78^{\circ} \mathrm{C}$. The bubble size is represented by the equivalent spherical diameter, $d_{b}$. Figure 1 shows their results for (a) $27^{\circ} \mathrm{C}$ and (b) $78^{\circ} \mathrm{C}$. As shown in the figure, for a given bubble size, $u_{b}$ tends to decrease with increasing pressure at both temperatures. They found that the effects of pressure and temperature, or more directly, the effects of physical properties of the gas and liquid phases on the variation of $u_{b}$ with $d_{b}$ could be represented or predicted most generally by the Fan-Tsuchiya equation (Fan and Tsuchiya, 1990) among three predictive equations. The other two are the modified Mendelson's wave-analogy equation (Mendelson, 1967) by Maneri (1995) and a correlation proposed by Tomiyama et al. (1995).

The Fan-Tsuchiya equation, generalized for high-pressure systems, can be written in a dimensionless form:

$$
u_{b}^{\prime}=u_{b}\left(\frac{\rho_{l}}{\sigma g}\right)^{1 / 4}=\left\{\left[\frac{M o^{-1 / 4}}{K_{b}}\left(\frac{\Delta \rho}{\rho_{l}}\right)^{5 / 4} d_{b}^{\prime 2}\right]^{-n}+\left[\frac{2 c}{d_{b}^{\prime}}+\left(\frac{\Delta \rho}{\rho_{l}}\right) \frac{d_{b}^{\prime}}{2}\right]^{-n / 2}\right\}^{-1 / n}
$$

where the dimensionless bubble diameter is given by

$$
d_{b}^{\prime}=d_{b}\left(\rho_{l} g / \sigma\right)^{1 / 2} \text {. }
$$

Three empirical parameters, $n, c$, and $K_{b}$, in Eq. (1) reflect three specific factors governing the rate of bubble rise. They relate to the contamination level of the liquid phase, to the varying dynamic effects of the surface tension, and to the viscous nature of the surrounding medium. The suggested values of these parameters are:

$$
\begin{gathered}
n=\left\{\begin{array}{ll}
0.8 & \text { for contaminated liquids } \\
1.6 & \text { for purified liquids }
\end{array},\right. \\
c=\left\{\begin{array}{ll}
1.2 & \text { for monocomponent liquids } \\
1.4 & \text { for multicomponent liquids }
\end{array},\right. \text { and } \\
K_{b}=\max \left(K_{b 0} M o^{-0.038}, 12\right)
\end{gathered}
$$

where

$$
K_{b 0}=\left\{\begin{array}{ll}
14.7 & \text { for aqueous solutions } \\
10.2 & \text { for organic solvents } / \text { mixtures }
\end{array} .\right.
$$


The modified Mendelson's equation is a special form of the Fan-Tsuchiya equation where the viscous term, i.e. the first term on the right side of Eq. (1), is omitted. Equally general as the FanTsuchiya equation for bubbles in liquids, the correlation by Tomiyama et al. (1995), which is given in terms of drag coefficient,

$$
C_{D}=\frac{4}{3} \frac{g \Delta \rho d_{b}}{\rho_{l} u_{b}^{2}}
$$

consists of three equations under different system purity:

$$
C_{D}=\max \left\{\min \left[\frac{16}{R e}\left(1+0.15 R e^{0.687}\right), \frac{48}{R e}\right], \frac{8}{3} \frac{E o}{E o+4}\right\}
$$

for purified systems;

$$
C_{D}=\max \left\{\min \left[\frac{24}{R e}\left(1+0.15 R e^{0.687}\right), \frac{72}{R e}\right], \frac{8}{3} \frac{E o}{E o+4}\right\}
$$

for partially contaminated systems; and

$$
C_{D}=\max \left[\frac{24}{R e}\left(1+0.15 R e^{0.687}\right), \frac{8}{3} \frac{E o}{E o+4}\right]
$$

for sufficiently contaminated systems. In Eqs (1) and (5), the dimensionless groups are defined as

$$
\begin{gathered}
M o=\frac{g \Delta \rho \mu_{l}^{4}}{\rho_{l}^{2} \sigma^{3}}, \\
\operatorname{Re}=\frac{d_{b} u_{b} \rho_{l}}{\mu_{l}}, \text { and } \\
\text { Eo }=\frac{g \Delta \rho d_{b}^{2}}{\sigma}
\end{gathered}
$$

where $\Delta \rho=\rho_{l}-\rho_{g}$. It is noted that $u_{b}$ can be obtained explicitly from Eq. (1) for a given $d_{b}$ as well as gas and liquid physical properties, while it can only be obtained implicitly from Eq. (5).

For predictions included in Fig. 1, measured values of physical properties under various operating pressures and temperatures (Lin and Fan, 1997; Lin et al., 1998) are used. As shown in the figure, the modified Mendelson equation, which is valid only under the inviscid condition, provides limited agreement between the measured and calculated results at the low temperature [Fig. 1(a)], suggesting that viscous forces predominate in the bubble rise process. On the other hand, at the high temperature [Fig. 1(b)], there is a strong agreement over the bubble size range of $d_{b}>2 \mathrm{~mm}$ including the sharp breakpoint/peak. This indicates that the liquid used tends to behave as a pure inviscid liquid. Note that over the pressure range from 0.1 to $19.4 \mathrm{MPa}$, the liquid viscosity varies from 29 to $48 \mathrm{mPa} \cdot \mathrm{s}$ at $27^{\circ} \mathrm{C}$, whereas it is almost constant within a range from 4.7 to $5.2 \mathrm{mPa} \cdot \mathrm{s}$ at $78^{\circ} \mathrm{C}$ (Lin et al., 1998 ). 
The Fan-Tsuchiya equation, Eq. (1), applied for the given liquid, a pure $(n=1.6)$, multicomponent ( $c$ $=1.4)$ organic solvent $\left(K_{b 0}=10.2\right)$, demonstrates good overall predictive capability except for the sharp peak existing for the high-temperature data [Fig. 1(b)]. The equation by Tomiyama et al. (1995) also has good general applicability, especially around the peak behavior occurring near $d_{b}=$ $2 \mathrm{~mm}$ at $78^{\circ} \mathrm{C}$; however, it tends to underestimate the $u_{b}$ values over the rest of the $d_{b}$ range.

The consistent difference in $u_{b}$ prevailing between 0.1 and 19.4 MPa for $d_{b}>2 \mathrm{~mm}$ is due to the significant increase in gas density (as large as 200-fold increase with pressure from 0.1 to 19.4 MPa). The density effect is accounted for in Eq. (1) in terms of $\Delta \rho / \rho_{l}$ or in Eq. (5) in terms of both $\Delta \rho / \rho_{l}$ and Eo. As can be seen from the equations and figure, the density difference between the continuous liquid phase and the dispersed gas phase plays an important role in determining $u_{b}$, especially for large bubbles.

Figure 2 shows the $R e-E o$ relationship often utilized in representing the general rise characteristics of single bubbles in liquids (Clift et al., 1978; Bhaga and Weber, 1981). The thin, background lines signify the general, quantitative trend for the rise velocity of single bubbles in purified Newtonian liquids under ambient conditions, plotted with constant intervals of log Mo. The figure shows the general agreement in correlation predictions. The experimental results under four conditions (Lin et al., 1998) are plotted in the figure. By employing accurate values for physical properties of the liquid phase and the gas density at given pressures and temperatures, the experimental results can be successfully represented over the entire Eo range, i.e. bubble size range, by Eq. (1). The prediction is proven to represent experimental data for various liquids under ambient conditions within some deviations (Tsuchiya et al., 1997). Furthermore, the single bubble rise velocity at high pressures can be reasonably estimated by incorporating the physical properties of the gas and liquid under the operating conditions.

\subsubsection{In liquid-solid suspensions}

Figure 3 shows the effect of pressure on the bubble rise velocity in a fluidized bed with Paratherm NF heat transfer fluid and $0.88-\mathrm{mm}$ glass beads at (a) $26.5^{\circ} \mathrm{C}$ and (b) $87.5^{\circ} \mathrm{C}$ (Luo et al., 1997b). At both temperatures, the bubble rise velocity decreases with an increase in pressure for a given solids holdup. The extent of the reduction is as high as by $50 \%$ from 0.1 to $17.3 \mathrm{MPa}$. A more drastic reduction in $u_{b}$, however, arises from the addition of solid particles. While the particle effect is small at low solids holdup $\left(\varepsilon_{s}<0.4\right)$, the effect is appreciable at high solids holdup $\left(\varepsilon_{s}=0.545\right)$, especially for high liquid viscosity [Fig. 3(a)]. A comparison of the data at $26.5^{\circ} \mathrm{C}$ and $87.5^{\circ} \mathrm{C}$, for 
the same $\varepsilon_{s}$ of 0.545 , indicates that the viscosity effect appears to be significant. The reduction of the bubble rise velocity with an increase in pressure can lead to a significant increase in the gas holdup of three-phase fluidized beds. The extent of the increase in gas holdup was reported to be around $100 \%$ at all gas velocities when the pressure is increased from 0.1 to $15.6 \mathrm{MPa}$ (Luo et al., 1997a). By comparing the pressure effect on the gas holdup with that on the bubble rise velocity, the increase in gas holdup with pressure is a consequence of the decreases in both the bubble size and the bubble rise velocity.

Similar plots are shown in Fig. 4 for the fluidized bed containing 0.21-mm glass beads (Luo et al., 1997b). While the extent of decrease in bubble rise velocity with an increase in pressure is comparable between 0.88 - and 0.21 - $\mathrm{mm}$ glass beads, the extent of decrease in bubble rise velocity with an increase in solids holdup is much smaller for the smaller particles. By comparing the corresponding data in Figs 3 and 4, this difference in the sensitivity of $u_{b}$ reduction to solids holdup variation is clearly shown for the high solids holdup cases.

The decrease in bubble rise velocity occurs due to corresponding variations of gas and liquid properties with pressure. In the presence of solid particles, it can be assumed, as a first approximation, that the particles modify only homogeneous properties of the surrounding medium. Luo et al. (1997b) examined the applicability of this homogeneous approach. The calculated results based on the Fan-Tsuchiya equation, Eq. (1), for $u_{b}$ are also plotted in Figs 3 and 4, where Eq. (1) is extended to liquid-solid suspensions by replacing the liquid properties, $\rho_{l}$ and $\mu_{l}$, by the effective properties of the liquid-solid suspension, $\rho_{m}$ and $\mu_{m}$ (Tsuchiya et al., 1997), respectively. The effective density can be estimated by

$$
\rho_{m}=\rho_{l}\left(1-\varepsilon_{s}\right)+\rho_{s} \varepsilon_{s} .
$$

The calculated results with constant values of $\mu_{m}$ given in Figs 3 and 4 are obtained by coupling Eq. (1) with the following relationship proposed by Tsuchiya et al. (1997) for the effective viscosity of liquid-solid suspensions:

$$
\frac{\mu_{m}}{\mu_{l}}=\exp \left[\frac{K \varepsilon_{s}}{1-\left(\varepsilon_{s} / \varepsilon_{s c}\right)}\right]
$$

with two parameters correlated by Luo et al. (1997b):

$$
\begin{aligned}
& K=\frac{3.1-1.4 \tanh \left[0.3\left(10-10^{2} u_{t}\right)\right]}{\phi} \text { and } \\
& \varepsilon_{s c}=\left\{1.3-0.1 \tanh \left[0.5\left(10-10^{2} u_{t}\right)\right]\right\} \varepsilon_{s 0}
\end{aligned}
$$


where $u_{t}$ is in m/s. The ranges of applicability of Eqs (8) and (9) are: $840<\rho_{l}<1000 \mathrm{~kg} / \mathrm{m}^{3} ; 1<\mu_{l}$ $<47 \mathrm{mPa} \cdot \mathrm{s} ; 19<\sigma<73 \mathrm{mN} / \mathrm{m} ; 0<\varepsilon_{s}<0.95 \varepsilon_{s 0} ; 7.9 \times 10^{-4}<u_{t}<0.26 \mathrm{~m} / \mathrm{s} ; 0.88<\phi \leq 1 ;$ and 0.56 $<\varepsilon_{s 0}<0.61$.

Equation (1) with parametric values of $\mu_{m}$, estimated from Eq. (8) under given conditions, predicts reasonably well the general trend exhibited by the reported data. However, a detailed match between the calculated and experimental results appears to be difficult to attain by assigning a constant value of $\mu_{m}$ under each condition. A more elaborate analysis is required to account for the effect of bubble size on interactions of the bubble with the surrounding medium (non-Newtonian approach) or with individual particles (heterogeneous approach).

\subsection{Heterogeneous approach: Discrete-phase computation}

Jean and Fan (1990) developed a mechanistic model that accounts for impact forces on a rising bubble due to particles. The model can predict the bubble rise velocity for small particles $\left(d_{p}<500\right.$ $\mu \mathrm{m})$, low-to-intermediate solids holdups $\left(\varepsilon_{s}<0.45\right)$ and large spherical-cap bubbles $\left(d_{b}>15 \mathrm{~mm}\right)$. It is desired to extend their model to cover the range of smaller bubble sizes as well. This was conducted by Luo et al. (1997b) with partial success based on a force balance on a rising bubble involving the net gravity, liquid drag, and particle-bubble collision forces.

A much more thorough scheme of prediction of a single bubble rising in a liquid-solid fluidized bed has recently been developed by Zhang et al. (1998b) using a two-dimensional discrete-phase simulation model for gas-liquid-solid fluidization systems. In this model, the volume-averaged method, the dispersed particle method (DPM) and the volume-of-fluid (VOF) method are used to account for the flow of liquid, solid, and gas phases, respectively. A bubble induced force (BIF) model, a continuum surface force (CSF) model, and Newton's third law are applied to account for the couplings of particle-bubble (gas), gas-liquid, and particle-liquid interactions, respectively. A close distance interaction (CDI) model (Zhang et al., 1998a) is included in the particle-particle collision analysis, which considers the liquid interstitial effects between colliding particles.

\subsubsection{Liquid-phase model}

The governing equations for the continuous phase of multiphase flows can be derived based on the Navier-Stokes equations for single-phase flows. Considering the existence of dispersed particles, a volume-averaging technique is used to develop a set of partial differential equations to describe the mass and momentum conservation of the liquid phase. The continuity equation for the liquid phase can be given as 


$$
\frac{\partial \varepsilon_{l}}{\partial t}+\nabla \cdot\left(\varepsilon_{l} v\right)=0
$$

The momentum equation for the liquid phase is

$$
\rho_{l} \frac{\partial\left(\varepsilon_{l} v\right)}{\partial t}+\rho_{l} \nabla \cdot\left(\varepsilon_{l} v v\right)=-\nabla p+\varepsilon_{l} \nabla \cdot \tau+\varepsilon_{l} \rho_{l} g+f_{b}
$$

where $v$ is the liquid velocity vector, $\varepsilon_{l}$ is the liquid holdup, $\rho_{l}$ is the liquid density, $p$ is the scalar pressure, $\tau$ is the viscous stress tensor, $\boldsymbol{g}$ is the gravitational acceleration, and $f_{b}$ is the total volumetric body force acting on the liquid phase other than the gravity force.

The Newtonian viscous stress tensor is used which is given as

$$
\tau=2 \mu S=\mu\left[(\nabla v)+(\nabla v)^{T}\right]
$$

where $\boldsymbol{S}$ is the rate-of-strain tensor and $\mu$ is the coefficient of dynamic viscosity.

\subsubsection{Gas-phase model}

Under high-pressure conditions, the effects of gas density and viscosity on the flow behavior would be significant. The simulation model for high pressures is conducted by including the simulation of the flow inside the gas bubble. The flow inside the gas bubble is governed by single-phase NavierStokes equations. Because of the difficulty of numerical calculation due to the discontinuous jump of properties across the interface between the gas bubble and the liquid-solid suspension, a continuous transition method (CTM) is employed. In this method, the discontinuous characteristics are replaced by a smooth variation of the properties (e.g., density and viscosity) from one phase to another within the finite interface thickness. The continuous transition method can overcome the problem of numerical divergence while simulating the flow field at both sides of the interface where the physical properties of the fluids strongly differ. By using the high-pressure fluid conditions, the resulting discrete phase simulation method can reveal the pressure effects on the variation of the flow characteristics in a gas-liquid-solid fluidization system.

The scalar fraction function, $\alpha(\boldsymbol{x}, t)$, solved by the VOF method (Hirt and Nichols, 1981) is used to construct this continuous transition function, and the fluid property at the interface can be given by:

$$
Q=Q_{m}^{*} \alpha(x, t)+Q_{g}^{*}[1-\alpha(x, t)]
$$

where $Q$ represents a property of the fluid, $Q_{m}^{*}$ and $Q_{g}^{*}$ represent the properties of liquid-solid suspension and gas bubble, respectively. By definition, $\alpha(\boldsymbol{x}, t)=1$ in the liquid or liquid-solid mixture, $0<\alpha(\boldsymbol{x}, t)<1$ at the free surface, and $\alpha(\boldsymbol{x}, t)=0$ in the gas bubble. Therefore, $Q$ is replaced by $Q_{m}^{*}$ or $Q_{g}^{*}$ when $\alpha(x, t)$ equals 1 in the liquid-solid suspension or equals 0 in the gas bubble. 
The advection equation for $\alpha(\boldsymbol{x}, t)$ is

$$
\frac{\partial \alpha}{\partial t}+(v \cdot \nabla) \alpha=0
$$

On the gas-liquid free surfaces, the stress boundary condition follows the Laplace equation as

$$
p_{s}=p-p_{v}=\sigma \kappa
$$

where the surface pressure, $p_{s}$, is the surface tension-induced pressure jump across a fluid interface. The continuum surface force (CSF) model (Brackbill et al., 1992) converts the surface force into a volume force within free surfaces. The volume force at the free surfaces is given by the CSF model as

$$
f_{s v}(x, t)=\sigma \kappa(x, t) \nabla \alpha(x, t) .
$$

This volume force is added to the volumetric body force term, $f_{b}$, in the momentum equation at the free surfaces.

\subsubsection{Dispersed particle method}

The motion of a particle in a flow field can be described in Lagrangian coordinates with its origin attached to the center of the moving particle. The motion of a single particle can be described by its acceleration and rotation in a nonuniform flow field. The particle accelerating in the liquid is governed by Newton's second law of motion as

$$
m_{p} \frac{d \boldsymbol{v}_{p}}{d t}=\boldsymbol{F}_{\text {total }} .
$$

The forces acting on a particle include interface forces between the fluid and particle, and forces imposed by external fields. The total force acting on a particle is composed of all applicable forces, including drag, added mass, gravity/buoyancy, Magnus force, Basset force, and other forces:

$$
\boldsymbol{F}_{\text {total }}=\boldsymbol{F}_{D}+\boldsymbol{F}_{A M}+\boldsymbol{F}_{G / B}+\boldsymbol{F}_{\text {Magnus }}+\boldsymbol{F}_{\text {Basset }}+\sum_{i} \boldsymbol{F}_{i}
$$

The general scheme of a stepwise molecular dynamic (MD) simulation (Allen and Tildesley, 1987), based on a predictor-corrector algorithm, is used to compute the particle motion. The hard sphere approach is used for the collision dynamics. The normal velocity and momentum changes of colliding particles are determined by a collinear collision model developed by Zhang et al. (1998a). The model includes the detailed close-range particle-fluid and particle-particle interactions during the entire process of particle collision. The tangential velocity and momentum changes are formulated and calculated based on a sticking/sliding model. 


\subsubsection{Coupling among individual phases}

When particles move into the gas-liquid interface, i.e. into the domain where $0.5<\alpha(x, t)<1$, the surface tension force is also acting on the particle. This force equals the volumetric surface tension force, $f_{s v}$, of Eq. (16) multiplied by the particle volume. If the total force of the particle is larger than the surface tension force, the particle would penetrate the bubble surface. The penetrating particle breaks the bubble surface momentarily upon contact. If the penetrating particle is small, the bubble may recover its original shape upon particle penetration (Chen and Fan, 1989). However, if there are several particles colliding with the bubble surface simultaneously, the resulting force may cause bubble breakage.

Based on Newton's third law of motion, the forces acting on particles from the liquid phase, which include $\boldsymbol{F}_{D}, \boldsymbol{F}_{A M}$, and $\boldsymbol{F}_{\text {Basset }}$, yield a reaction force on the liquid. Therefore, the momentum transfer from particles to liquid is taken into account by adding the volumetric liquid-particle interaction force to the body force term, $f_{b}$, in Eq. (11).

The liquid properties on the particle surface are obtained by an area-weighted averaging based on the properties at the four grid points of the computational cell containing the particle. The liquid holdup, $\varepsilon_{l}$, is obtained by subtracting the volume fraction of the particles in the computational cell. However, this cell-averaged liquid holdup is only used for solving the volume-averaged equations of liquid phase. When accounting for the liquid holdup effect on the particle drag coefficient in the liquid-solid medium, a particle-centered area averaging method is used for the calculation.

\subsection{Computational results: Single bubble rising}

The simulations of representative cases, i.e. a single bubble rising and particle entrainment by a bubble in a liquid-solid fluidized bed under ambient conditions (Zhang et al., 1998b), and a single bubble rising in a liquid under high-pressure conditions, are presented in this section.

\subsubsection{Ambient conditions}

Comparisons of the simulation and the experimental results of a single bubble rising in a liquid-solid suspension are shown in Fig. 5. The simulation domain is $30 \times 80 \mathrm{~mm}^{2}$ and a computational grid size is $0.15 \times 0.16 \mathrm{~mm}^{2}$. One thousand particles with a density of $2,500 \mathrm{~kg} / \mathrm{m}^{3}$ and a diameter of $1.0 \mathrm{~mm}$ are used as the solid phase. An aqueous glycerin solution $(80 \mathrm{wt} \%)$ with $\rho_{l}=1,206 \mathrm{~kg} / \mathrm{m}^{3}, \mu_{l}=52.9$ $\mathrm{mPa} \cdot \mathrm{s}$, and $\sigma=62.9 \mathrm{mN} / \mathrm{m}$ is used as the liquid phase. A circular bubble with a diameter of $10 \mathrm{~mm}$ is initially imposed in the computational domain with its center $15 \mathrm{~mm}$ above the bottom. Initially, the particles are randomly positioned in a $30 \times 240 \mathrm{~mm}^{2}$ area. Then, the simulation is performed for 
particles settling at a liquid velocity of $5 \mathrm{~mm} / \mathrm{s}$. At this stage, the bubble is treated as an obstacle and fixed in the original place. An equilibrium bed height is reached at $80 \mathrm{~mm}$, which gives a threedimensional equivalent solids holdup of 0.44. After the bed reaches its equilibrium height, the simulation is restarted with bubble tracking and particle movement. The time step of simulation for liquid and solid phases is $5 \mu \mathrm{s}$. Experiments are performed in a two-dimensional column with a thickness of $7 \mathrm{~mm}$. The solids holdup, liquid velocity, and the liquid and solids properties are the same as the simulation conditions. As shown in the figure, the simulation and experimental results of the bubble rise velocity and the bubble shape generally agree well.

By closely following the evolution of the particle flow around a single bubble, the mechanisms of particle entrainment in a liquid-solid suspension were studied by Miyahara et al. (1989), Fan and Tsuchiya (1990), and Tsuchiya et al. (1992). These studies indicated that particles are drawn from the upper surface of the suspension into the freeboard of liquid in the wake behind the bubble, and particle-containing vortices are shed from the wake in the freeboard. The simulation results of the bubble emerging from the bed surface are shown in Fig. 6. As seen in subsequent frames of Fig. 6, a group of particles are dragged by the bubble wake. An agreement in spatial and temporal variations of the solid particles in the entrainment process with the rising bubble is found between the simulation and the experimental results by Miyahara et al. (1989) and Tsuchiya et al. (1992).

\subsubsection{High-pressure conditions}

A single bubble rising in a liquid at elevated pressures $(P=19.4 \mathrm{MPa})$ is simulated. The properties of the liquid phase under ambient conditions are: $\rho_{l}=868 \mathrm{~kg} / \mathrm{m}^{3} ; \mu_{l}=29 \mathrm{mPa} \cdot \mathrm{s}$; and $\sigma=30 \mathrm{mN} / \mathrm{m}$. The computational domain is $100 \times 90 \mathrm{~mm}^{2}$ with $90 \times 90$ grids. A circular nitrogen bubble with a diameter of $80 \mathrm{~mm}$ is initially imposed at $15 \mathrm{~mm}$ from the bottom, and its rising behavior is tracked by numerical simulation. The time step of simulation for the liquid and solid phases is $5 \mu$ s. Simulation results are shown in Fig. 7, in which the original point of the coordinate system is fixed on the mass center of the rising bubble. The numerical simulation indicates that the bubble rise velocity decreases with an increase in pressure, and is in good agreement with the experimental data and the prediction by the Fan-Tsuchiya equation, Eq. (1). It also can be seen in the figure that the elevated pressure causes the bubble shape to become more flat due to the variation of properties inside the bubble. As shown in Fig. 7, the simulation cannot only capture the wake structure, but also predict the internal flow circulation structure in the bubble.

\subsection{Bubble formation, initial bubble size, and jetting}


Numerous experimental and modeling studies have been conducted over the past decades on bubble formation from a single orifice or nozzle submerged in liquids, mostly under ambient conditions (Kupferberg and Jameson, 1969; Kumar and Kuloor, 1970; Azbel, 1981; Lin et al., 1994; Ruzicka et al., 1997). Among various factors that affect the bubble formation, the wettability of the orifice surface is an important factor, which affects the initial size of the bubble formed on the orifice. Lin et al. (1994) found that initial bubble size increases significantly with the contact angle between the bubble and the orifice surface when the contact angle exceeds the threshold value of $45^{\circ}$. Various models were established to predict the initial bubble size from a single nozzle in liquids. However, only a few studies were conducted at elevated pressures (LaNauze and Harris, 1974; Idogawa et al., 1987; Tsuge et al., 1992; Wilkinson and Van Dierendonck, 1994). The high-pressure studies indicated that an increase in gas density reduces the size of bubbles formed from a single orifice. However, these results were limited to water systems only. The pressure effect on the initial bubble size in hydrocarbon liquids is not fully understood. Furthermore, studies of the bubble formation in liquids in the presence of particles, as in slurry bubble columns and three-phase fluidized bed systems, are very limited. The experimental data of Massimilla et al. (1961) in an air-water-glass beads three-phase fluidized bed revealed that the bubbles formed from a single nozzle in the fluidized bed are larger in size than those in water, and the initial bubble size increases with the solids concentration. Yoo et al. (1997) investigated bubble formation in pressurized liquid-solid suspensions. They used $18.6 \mathrm{wt} \%$ aqueous glycerol solution and $0.1-\mathrm{mm}$ polystyrene beads as the liquid and solid phases, respectively. The densities of the liquid and the particles were identical, and thus, the particles were neutrally buoyant in the liquid. The results indicated that initial bubble size decreases inversely with pressure under otherwise constant conditions, i.e. gas flow rate, temperature, solids concentration, orifice diameter, and gas chamber volume. Their results also showed that the particle effect on initial bubble size is insignificant. The difference in the finding regarding the particle effects on initial bubble size between Massimilla et al. (1961) and Yoo et al. (1997) may possibly be due to the difference in particle density.

A mechanistic model is described to predict the initial bubble size in liquid-solid suspensions at high-pressure conditions (Luo et al., 1998c). The model considers various forces induced by the particles, and is an extension of a two-stage spherical bubble formation model developed by Ramakrishnan et al. (1969) for liquids. In the two-stage spherical bubble formation model, bubbles are assumed to be formed in two stages, namely, the expansion stage and the detachment stage. The bubble expands with its base attached to the nozzle during the first stage. In the detachment stage, the bubble base moves away from the nozzle, although the bubble remains connected with the nozzle 
through the neck. The shape of the bubble is assumed to remain spherical during the entire bubble formation process. It is also assumed in this model that a liquid film always exists around the bubble. During the expansion and detachment stages, particles collide with the bubble and stay on the liquid film. The particles and the liquid surrounding the bubble are set in motion as the bubble grows and rises.

The volume of the bubble at the end of the first stage and during the second stage can be described by considering a balance of all the forces acting on the bubble being formed if the instantaneous gas flow rate, $Q_{o}$, or the instantaneous gas velocity, $u_{o}$, through the orifice, is known. The forces induced by the liquid include the upward forces (effective buoyancy force, $F_{B}$, and gas momentum force, $F_{M}$ ), and the downward resistance (liquid drag, $F_{D}$, surface tension force, $F_{\sigma}$, bubble inertial force, $F_{I, g}$, and Basset force, $\left.F_{\text {Basset }}\right)$ as shown in Fig. 8. It is assumed that the particles affect the bubble formation process only through two additional downward forces on the bubble, i.e. the particle-bubble collision force, $F_{C}$, and the suspension inertial force, $F_{I, m}$, due to the acceleration of the liquid and particles surrounding the bubble. Therefore, the overall force balance on the bubble in this model can be written as

$$
F_{B}+F_{M}=F_{D}+F_{\sigma}+F_{\text {Basset }}+F_{I, g}+F_{C}+F_{I, m} .
$$

The expansion stage and the detachment stage follow the same force balance equation, Eq. (19), although the expression for the same force in the two stages may be different. The expressions for all the forces under two stages are listed in Table 1. The particle-bubble collision force is merely the rate of momentum change of the particles colliding with the bubble surface. The suspension inertial force is calculated from the suspension flow field around an accelerating bubble, obtained from a particle image velocimetry system.

The model is applied to simulate the bubble formation process under constant flow conditions, which are characterized by constant gas flow rate through the orifice. When the volume of the gas chamber is small, the bubble formation can normally be assumed under constant flow conditions. It can be seen from Fig. 9 that the model closely predicts experimental data on the initial bubble size in highpressure slurry systems (Luo et al., 1998c). Under constant flow conditions $(N c<1)$, the pressure effect is insignificant. Note that $N c$ is the dimensionless capacitance number and is equal to $4 V_{c} g \rho_{l} / \pi D_{o}^{2} P_{s}$.

In most industrial gas distributors, the gas chamber volume is large and the bubble formation process is under other conditions, e.g., constant pressure or intermediate conditions; in these cases, the 
orifice gas flow rate is not constant and depends on the pressure fluctuations in the chamber and in the bubble. The experimental study under such conditions is scarce. Yang et al. (1999) measured the initial bubble size in a slurry bubble column under intermediate conditions $(N c>1)$ using an optical fiber probe. As shown in Fig. 10, the pressure has a significant effect on the initial bubble size under these conditions (Yang et al., 1999). The initial bubble size decreases with an increase of pressure for the bubble formation with a large gas chamber. In order to model the bubble formation under such conditions, the pressure fluctuations in the gas chamber and in the bubble must be considered to account for the time-variant orifice gas flow rate as illustrated below.

The instantaneous gas flow rate through the orifice depends on the pressure difference in the gas chamber, $P_{c}$, and inside the bubble, $P_{b}$, as well as the flow resistance of the orifice, which can be described by the orifice equation as given in Eq. (20a). The pressure in the gas chamber can be evaluated by applying the first law of thermodynamics, considering an adiabatic compression process as given in Eq. (20b) (Wilkinson and Van Dierendonck, 1994). The pressure inside the bubble is governed by a modified Rayleigh's equation (Pinczewski, 1981). In order to simulate the bubble formation in liquid-solid suspensions, the effect of particles on the pressure inside the bubble must be considered. Yang et al. (1999) replaced the liquid inertial term in the modified Rayleigh equation with the suspension inertia, quantified based on the suspension flow field around an accelerating bubble obtained by the PIV measurement, as given in Eq. (20c).

$$
\begin{gathered}
\Delta P=\left|P_{c}-P_{b}\right|=\left(\frac{Q_{o}}{k_{o}}\right)^{2} . \\
\frac{\mathrm{d} P_{c}}{\mathrm{~d} t}=\frac{\gamma}{V_{c}}\left(P_{e} Q_{g}-P_{c} Q_{o}\right) . \\
P_{b}-P_{o}=\zeta \rho_{m}\left[\frac{r_{b}}{3} \frac{\mathrm{d}^{2} r_{b}}{\mathrm{~d} t^{2}}+\left(\frac{\mathrm{d} r_{b}}{\mathrm{~d} t}\right)^{2}\right]+\frac{2 \sigma}{r_{b}}+\frac{4 \mu_{l}}{r_{b}} \frac{\mathrm{d} r_{b}}{\mathrm{~d} t} .
\end{gathered}
$$

where $P_{o}$ is the hydrostatic pressure at the bubble surface. The three terms on the right-hand side of Eq. (20c) represent the contributions of inertial, surface tension, and viscous forces, respectively. The coefficient $\zeta$ in Eq. (20c) is equal to 3.86 for bubbles formed in liquid-solid suspensions (Luo et al., 1998c) and to 11/16 for bubbles formed in liquids, corresponding to the added mass in inviscid 
liquids (Milne-Thomson, 1955). Combining Eqs (19) and (20a, b, c), and solving these coupled ordinary differential equations simultaneously, the change of the initial bubble size with the time can be obtained. If a certain bubble detachment criterion is used, the initial bubble size can be estimated.

At a low gas velocity, discrete bubbles are formed. On the other hand, at a high gas velocity, jetting occurs and bubbles are formed from the top of the jet. The bubbles formed from a jet are of a wide size distribution. The empirical correlation provided by Idogowa et al. (1987) indicated that the bubbling-jetting transition velocity in a liquid is proportional to the gas density raised to the power of -0.8. Luo et al. (1998b) investigated the transition from bubbling to jetting under high pressures. They revealed a significant effect of the orifice Reynolds number, $R e_{o}=\rho_{g} D_{o} u_{o} / \mu_{g}$, on the bubbling and jetting phenomena. Photographs of the gas flow through an orifice in Paratherm NF heat transfer fluid at a high pressure for various $R e_{o}$ are shown in Fig. 11. At $R e_{0}=1,075$, single bubbles are formed from the orifice. With increasing $R e_{0}$ to 5,321, bubbles being formed at the orifice start to interact with the preceding ones. Bubble coalescence occurs between the two bubbles, sometimes involving more bubbles. At $R e_{0}=8,809$, frequent coalescence of successive bubbles is observed, i.e. the beginning of the bubbling-jetting transition. As $R e_{o}$ increases, the jetting regime becomes more apparent. Bubbles break away from the top of the jet. Moreover, the jet penetration depth increases with an increase in $R e_{0}$.

\subsection{Bubble coalescence}

For gas-liquid systems, the experimental results available in the literature indicate that an increase of pressure retards the bubble coalescence (Sagert and Quinn, 1977, 1978). There are three steps in the bubble coalescence process (Vrij, 1966; Chaudhari and Hoffmann, 1994): (1) approach of two bubbles to form a thin liquid film between them; (2) thinning of the film by the drainage of the liquid under the influence of gravity and suction due to capillary forces; and (3) rupture of the film at a critical thickness. The second step is the rate controlling step in the coalescence process and the bubble coalescence rate can be approximated by the film thinning rate (Vrij, 1966). The film thinning velocity can be expressed as (Sagert and Quinn, 1977, 1978)

$$
-\frac{\mathrm{d} l}{\mathrm{~d} t}=\frac{32 l^{3} \sigma}{3 \phi R_{d}^{2} \mu_{l} d_{b}}
$$

where the parameter $\phi$ is a measure of the surface drag or velocity gradient at the surface due to the adsorbed layer of the gas.

It is known that surface tension decreases and liquid viscosity increases with increasing pressure. In addition, $\phi$ increases with pressure. As seen from Eq. (21), all these variations contribute to the 
reduction of the film thinning velocity, and hence, the bubble coalescence rate, as pressure increases. As a result, the time required for two bubbles to coalesce is longer and hence the rate of overall bubble coalescence in the bed is reduced at high pressures. Moreover, the frequency of bubble collision decreases with increasing pressure. An important mechanism for bubble collision is bubble wake effects (Fan and Tsuchiya, 1990). When the differences in bubble size and bubble rise velocity are small at high pressures, the likelihood of small bubbles being caught and trapped by the wakes of large bubbles decreases. Therefore, bubble coalescence is suppressed by the increase in pressure, due to the longer bubble coalescence time and the smaller bubble collision frequency.

\subsection{Bubble breakup and maximum stable bubble size}

It is known that the variation of bubble size with pressure is the key to understanding pressure effects on hydrodynamics. The upper limit of the bubble size is set by the maximum stable bubble size, $D_{\max }$, above which the bubble is subjected to breakup and hence is unstable. Several mechanisms have been proposed for the bubble breakup phenomenon and based on these mechanisms, theories have been established to predict the maximum bubble size in gas-liquid systems.

Hinze et al. (1955) proposed that the bubble breakup is caused by the dynamic pressure and the shear stresses on the bubble surface induced by different liquid flow patterns, e.g., shear flow and turbulence. When the maximum hydrodynamic force in the liquid is larger than the surface tension force, the bubble disintegrates into smaller bubbles. The liquid Weber number can quantify this mechanism. When the Weber number is larger than a critical value, the bubble is not stable and disintegrates. This theory was adopted to predict the breakup of bubbles in gas-liquid systems (Walter and Blanch, 1986). Calculations by Lin et al. (1998) showed that the theory underpredicts the maximum bubble size and cannot predict the effect of pressure on bubble size.

A maximum stable bubble size exists for bubbles rising freely in a stagnant liquid without external stresses, e.g., rapid acceleration, shear stress, and/or turbulence fluctuations (Grace et al., 1978). The Rayleigh-Taylor instability has been regarded as the mechanism for bubble breakup under such conditions. A horizontal interface between two stationary fluids is unstable to disturbances with wavelengths exceeding a critical value if the upper fluid has a higher density than the lower one (Bellman and Pennington, 1954):

$$
\lambda_{c}=2 \pi \sqrt{\frac{\sigma}{g\left(\rho_{l}-\rho_{g}\right)}}
$$

Chen and Fan (1988) obtained an equation for a curved surface as in the case of bubble. Grace et al. (1978) modified the Rayleigh-Taylor instability theory by considering the time available for the 
disturbance to grow and the time required for the disturbance to grow to an adequate amplitude. Batchelor (1987) pointed out that the observed size of air bubbles in water was considerably larger than that predicted by the model of Grace et al. (1978). Batchelor (1987) further took into account the stabilizing effects of the liquid acceleration along the bubble surface and the non-constant growth rate of the disturbance. In Batchelor's model, the information of the magnitude of the disturbances is required for the prediction of the maximum bubble size; however, the magnitude of the disturbances is not known. The models based on the Rayleigh-Taylor instability predict an almost negligible pressure effect on the maximum bubble size; in fact, Eq. (22) implies that the bubble is more stable when the gas density is higher.

The Kelvin-Helmholtz instability is similar to the Rayleigh-Taylor instability, except that the former allows a relative velocity between the fluids, $u_{r}$. Using the same concept of Grace et al. (1978), Kitscha and Kocamustafaogullari (1989) applied the Kelvin-Helmholtz instability theory to model the breakup of large bubbles in liquids. Wilkinson and Van Dierendonck (1990) applied the critical wavelength to explain the maximum stable bubble size in high-pressure bubble columns:

$$
\lambda_{c}=\frac{2 \pi \sqrt{\frac{\sigma}{g\left(\rho_{l}-\rho_{g}\right)}}}{\frac{\rho_{l}}{\rho_{l}+\rho_{g}} \frac{\rho_{g} u_{r}^{2}}{2 \sqrt{\sigma g\left(\rho_{l}-\rho_{g}\right)}}+\sqrt{1+\frac{\rho_{l}^{2} \rho_{g}^{2} u_{r}^{4}}{4\left(\rho_{l}+\rho_{g}\right)^{2} \sigma g\left(\rho_{l}-\rho_{g}\right)}}} .
$$

Disturbances in the liquid with a wavelength larger than the critical wavelength can break up a bubble. Equation (23) indicates that the critical wavelength decreases with an increase in pressure and therefore bubbles are easier to disintegrate by disturbances at higher pressures. However, the critical wavelength is not equivalent to the maximum stable bubble size, and Eq. (23) alone cannot quantify the effect of pressure on bubble size.

All of the models mentioned above do not account for the internal circulation of the gas. The internal circulation velocity is of the same order of magnitude as the bubble rise velocity. A centrifugal force is induced by this circulation, pointing outwards toward the bubble surface. This force can suppress the disturbances at the gas-liquid interface and thereby stabilizing the interface. The centrifugal force may be another reason to explain the underestimation of $D_{\max }$ by the model by Grace et al. (1978). On the other hand, the centrifugal force can also disintegrate the bubble, as it increases with an increase in bubble size. The bubble breaks up when the centrifugal force exceeds the surface tension force, especially at high pressures when gas density is high. Levich (1962) assumed the centrifugal force to be equal to the dynamic pressure induced by the gas moving at the bubble rise velocity, i.e. $k_{f} \rho_{g} u_{b}^{2} / 2\left(k_{f} \approx 0.5\right)$, and proposed a simple equation to calculate the maximum stable bubble size: 


$$
D_{\max } \approx \frac{3.63 \sigma}{u_{b}^{2} \sqrt[3]{\rho_{l}^{2} \rho_{g}}} .
$$

Equation (24) severely underpredicts the maximum bubble size in air-water systems, although it shows a significant effect of pressure on the maximum bubble size. Considering all the theories proposed in the literature, the mechanism for bubble breakup at high pressures is still unknown.

An analytical criterion for the bubble breakup is derived by considering a single large bubble rising in a stagnant liquid or slurry at a velocity of $u_{b}$, without any disturbances on the gas-liquid interface. The bubble is subjected to breakup when its size exceeds the maximum stable bubble size due to the circulation-induced centrifugal force (Luo et al., 1998a). Large bubbles normally assume a spherical cap shape; in this work, the spherical-cap bubble is approximated by an ellipsoidal bubble with the same volume and the same aspect ratio (height to width). The circulation of gas inside the bubble can be described by Hill's vortex (Hill, 1894). To model the bubble breakup, it is necessary to evaluate the $x$-component of the centrifugal force, $F_{x}$, induced by the circulation on the entire bubble surface as shown in Fig. 12. A rigorous theoretical derivation from Hill's vortex yields the expression for $F_{x}$ :

$$
F_{x}=\frac{9 \pi \rho_{g} u_{b}^{2} a^{2}}{64 \sqrt{2} \alpha} .
$$

The surface tension force is the product of the surface tension and the circumference of the bubble,

$$
F_{\sigma}=\sigma L=\sigma \int_{\text {ellipse }} \sqrt{\left(\delta r_{c}\right)^{2}+(\delta z)^{2}}=4 \sigma a \mathrm{E}\left(\sqrt{1-\alpha^{2}}\right) .
$$

Also, the volume equivalent bubble diameter, $d_{b}$, is related to $a$ and $\alpha$ by

$$
a=\frac{d_{b}}{\sqrt[3]{8 \alpha}}
$$

Note that the centrifugal force is affected significantly by the gas density, the aspect ratio of the bubble, the bubble size, and the bubble rise velocity. The bubble is not stable if $F_{x}$ is larger than $F_{\sigma}$, i.e.

$$
u_{b}^{2} d_{b} \geq \frac{8 \alpha^{4 / 3} \mathrm{E}\left(\sqrt{1-\alpha^{2}}\right)}{0.312} \frac{\sigma}{\rho_{g}} .
$$

When the centrifugal force is larger than the surface tension force, the bubble should be stretched in the $x$ direction. During the stretching, the aspect ratio, $\alpha$, becomes smaller while $d_{b}$ and $u_{b}$ can be assumed to remain constant. As a result, the centrifugal force increases, the surface tension force decreases, and the bubble stretching becomes an irreversible process. The sequence of bubble images 
shown in Fig. 13 confirms the proposed mechanism of bubble breakup. The bubble images in the figure are obtained at a pressure of 3.5 MPa. Using the Davies-Taylor equation (Davies and Taylor, 1950) for the bubble rise velocity, the maximum stable bubble size is

$$
D_{\max } \approx 7.16 \alpha^{2 / 3} \mathrm{E}\left(\sqrt{1-\alpha^{2}}\right)^{1 / 2} \sqrt{\frac{\sigma}{g \rho_{g}}} .
$$

The simplified forms of Eq. (29) are (Luo et al., 1998a):

$$
D_{\max } \approx 2.53 \sqrt{\frac{\sigma}{g \rho_{g}}} \quad(\text { for } \alpha=0.21)
$$

in liquids, and $D_{\max } \approx 3.27 \sqrt{\frac{\sigma}{g \rho_{g}}} \quad($ for $\alpha=0.3)(30 \mathrm{~b})$

in liquid-solid suspensions. Further, based on the Davies-Taylor equation, the rise velocity of the maximum stable bubble is

$$
u_{\max }=C\left(\frac{\sigma g}{\rho_{g}}\right)^{1 / 4}
$$

where $C$ is a constant. The comparison between experimental data and the predictions of Eq. (30) and by other instability theories is shown in Fig. 14(a). The figure indicates that the proposed model can explain the observed effect of pressure on the bubble size. It is clear that the internal circulation model captures the intrinsic physics of bubble breakup at high pressures. The comparison of the predictions by different models indicates that the bubble breakup is governed by the internal circulation mechanism at high pressures over $10 \mathrm{~atm}$, whereas the Rayleigh-Taylor instability or Kelvin-Helmholtz instability is the dominant mechanism at low pressures. Based on the experimental results at elevated pressures, in which the bubble rise velocity is noted to be proportional to $\rho_{g}^{-0.5}$, Letzel et al. (1998) concluded that the Kelvin-Helmholtz theory governs the bubble instability. However, this proportional relationship between the bubble rise velocity and the gas density should be perceived to be only as a sufficient condition, but not as a necessary condition. This proportional relationship is not necessarily required to be held for a constant square of the growth factor of the disturbance in the Kelvin-Helmholtz theory as the critical wave number may vary with flow conditions under different gas densities. Figure 14(b) presents experimental data and correlation or model predictions of bubble velocity or bubble swarm velocity by various investigators (Davenport et al., 1967; El-Temtamy and Epstein, 1980; Schumpe and Grund, 1986; Wilkinson and Van Dierendonck, 1990; Yu and Kim, 1991; Grund et al., 1992; Wilkinson et al., 1992; Liu and Bankoff, 1993; Hyndman et al., 1997; Letzel et al., 1997, 1998; Luo et al., 1998a) 
under various operating conditions for air (or nitrogen)-water systems. Relevant information on bubble or bubble swarm velocities in air-water systems regarding these investigations is given in Table 2. It is seen in the figure that bubble or bubble swarm velocities decrease with an increase in gas density or gas pressure at low gas densities, and this effect is substantially less pronounced at high gas densities. It is found that there is an appreciable variation of the bubble or bubble swarm velocities at low gas densities under various conditions. However at high gas densities, the variation of these velocities appears to be small and these velocities are within the range of prediction of the mechanistic model for high pressures developed by Luo et al. (1998a).

\section{Macroscopic Hydrodynamics}

\subsection{Moving packed bed phenomenon}

For three-phase fluidization systems involving large particles, two striking phenomena pertaining to macroscopic hydrodynamic behavior are bed contraction and moving packed bed flow. Bed contraction is characterized by a decrease in the bed height of a liquid-solid fluidized bed when a low velocity of gas is introduced to the bed. The bed contraction is caused by the behavior of bubble wake, which entraps liquid and particles and therefore is associated with large bubble systems. The entrainment of the liquid and particles by the bubble wake reduces the effective amount of liquid in the bed used to fluidize the remaining particles. The bed contraction phenomenon has been extensively studied under ambient fluidization conditions (Massimilla et al., 1959; Ostergaard, 1964; El-Temtamy and Epstein, 1979). At high pressures, such a phenomenon has also been observed to occur (Jiang et al., 1997).

The moving packed bed flow is characterized by the motion of solids in piston flow in a three-phase fluidized bed. The moving packed bed flow, which usually occurs during the start-up of the bed, depends not only on the gas and liquid velocities, but also on how they are introduced to the bed. It is caused by the surface phenomena involving fine bubbles attached onto particles and subsequent formation of a fine bubble blanket under the packed solids; a liquid flow would move the entire bed upward. This phenomenon is thus associated with the small bubble system. The moving packed bed flow in a three-phase fluidized bed is a known, anomalous event in the resid hydrotreating industry. It was observed in the $1960 \mathrm{~s}$ in the bench and pilot units during the development and commercialization of the resid hydrotreating process (Fan, 1999). The reactor was typically operated at pressures between 5.5 and $21 \mathrm{MPa}$ and at temperatures between $300^{\circ} \mathrm{C}$ and $425^{\circ} \mathrm{C}$. In the early 1970s, the moving packed bed flow was observed in a commercial three-phase fluidized bed reactor. The occurrence of the moving packed bed in a three-phase fluidized bed could simply be 
circumvented by utilizing a start-up procedure that involves degassing the bed first and then introducing liquid flow to expand the bed prior to commencing the gas flow. Commercial operators of three-phase fluidized bed reactors have long recognized and undertaken a proper start-up procedure of this nature since observing this anomalous event. As the small bubbles can also be generated under the ambient conditions using surfactants in an air-water system, the moving packed bed flow was reported in open literature first by Saberian-Broudjenni et al. (1984) and later by Bavarian and Fan (1991a, b) in small columns with small bubbles generated in such manner.

\subsection{Flow regime transition}

Three flow regimes can be identified based on the bubble flow behavior in bubble columns and slurry bubble columns: the dispersed bubble (or homogeneous bubble flow), the coalesced bubble (or churn-turbulent flow), and the slugging regimes. In the homogeneous bubble flow regime, no bubble coalescence occurs and the bubbles are of uniform, small size. The homogeneous bubble flow regime predominates at high liquid velocities and at low and intermediate gas velocities. At low liquid and high gas velocities, either the churn-turbulent flow or slugging regime occurs depending on the column diameter. In columns of large diameter, the churn-turbulent flow regime always occurs at high gas velocities. In this regime, bubbles tend to coalesce and both bubble size and bubble rise velocity become large and show a wide distribution.

The knowledge of the transition between the homogeneous bubble flow and the churn-turbulent flow regimes is important for the design and operation of industrial reactors. The transition velocity depends on gas distributor design, physical properties of the phases, operating conditions, and column size. The flow regimes and the regime transition have been studied extensively under ambient conditions over the last three decades (Wallis, 1969; Joshi and Lali, 1984; Shnip et al., 1992; Tsuchiya and Nakanishi, 1992; Zahradnik et al., 1997). Most of these studies pointed out a critical role played by the liquid-phase turbulence during the regime transition, and employed phenomenological models to predict the flow transition from the homogeneous regime to the heterogeneous regime. The effect of the operating pressure on the regime transition has been examined by many researchers in bubble columns (Tarmy et al., 1984; Clark, 1990; Krishna et al., 1991, 1994; Wilkinson et al., 1992; Hoefsloot and Krishna, 1993; Reilly et al., 1994; Letzel et al., 1997; Lin et al., 1999b), in three-phase fluidized beds (Luo et al., 1997a), and in slurry bubble columns (Clark, 1990).

Letzel et al. (1997) studied the influence of pressure on the stability of bubbly flows in a bubble column with the nitrogen-water system by using the stability theory of Batchelor (1988) and 
Lammers and Biesheuvel (1996). They found that a higher gas density has a stabilizing effect on the flow and that the gas fraction at the instability point increases with gas density, while the gas velocity at the instability point only slightly increases with gas density. However, the conclusion is limited to a narrow range of operating pressures $(0.1$ to $1.3 \mathrm{MPa})$. Lin et al. (1999b) used the standard deviation of the pressure fluctuation and the drift flux model to identify the flow transition from the homogeneous regime to the heterogeneous regime in a bubble column using nitrogen and Paratherm NF heat transfer fluid at pressures up to $15.2 \mathrm{MPa}$ and temperatures up to $78^{\circ} \mathrm{C}$. It was found that increasing pressure or temperature delays the regime transition as shown in Fig. 15(a).

Wilkinson et al. (1992) proposed a correlation to estimate the gas holdup and gas velocity at the transition point under high-pressure conditions. This predictive scheme incorporates the concept of bimodal bubble size distribution presented by Krishna et al. (1991), i.e. the churn-turbulent regime is characterized by a bimodal bubble size distribution, consisting of fast rising large bubbles $(>5 \mathrm{~cm}$ in diameter) and small bubbles (typically, $<5 \mathrm{~mm}$ in diameter). Wilkinson et al. (1992) found that the transition velocity depends on the liquid properties and can be estimated by the following correlations:

$$
\begin{gathered}
\varepsilon_{g, \text { tran }}=\frac{U_{g, \text { tran }}}{u_{\text {small }}}=0.5 \exp \left(-193 \rho_{g}{ }^{-0.61} \mu_{l}^{0.5} \sigma^{0.11}\right) \text {, and } \\
u_{\text {small }}=2.25\left(\frac{\sigma}{\mu_{l}}\right)\left(\frac{\sigma^{3} \rho_{l}}{g \mu_{l}^{4}}\right)^{-0.273}\left(\frac{\rho_{l}}{\rho_{g}}\right)^{0.03}
\end{gathered}
$$

where $u_{\text {small }}$ is the rise velocity of small bubbles. As shown in Fig. 15(a), a reasonable agreement for the regime transition velocity can be obtained between the experimental data obtained by Lin et al. (1999b) and the correlation of Wilkinson et al. (1992) when the in-situ physical properties of the fluids at a given temperature and pressure are used in the correlation.

The studies of the regime transition in three-phase fluidized beds and slurry bubble columns are scarce. Luo et al. (1997a) studied the transition velocity in a three-phase fluidized bed over a pressure range of 0.1 to $15.6 \mathrm{MPa}$ by analyzing the drift flux of gas. Two types of glass beads of 2.1 and $3 \mathrm{~mm}$ in diameter are used as the solid phase. The drift flux of gas increases with the gas holdup in the dispersed regime; in the coalesced bubble regime, the rate of increase is much larger. As the pressure increases, the transition gas velocity and the gas holdup at the transition point increase, under all the particle size and liquid velocity conditions. The pressure effect on the regime transition is significant, but the effect levels off at a pressure around $6 \mathrm{MPa}$ as shown in Fig. 15(b). The experimental study also shows that the transition velocity increases with liquid velocity and slightly increases with particle size, similar to the regime transition behavior at ambient conditions. Clark 
(1990) studied the regime transition in a hydrogen-methanol-catalyst system at pressures between 2.5 and $10 \mathrm{MPa}$ and temperatures from $20^{\circ} \mathrm{C}$ to $180^{\circ} \mathrm{C}$. Glass beads with a particle size range of 45 to $63 \mu \mathrm{m}$ were used as the solid catalyst. It was found that the addition of fine particles to the liquid phase promotes bubble coalescence, which accelerates the transition to the churn-turbulent regime. However, the regime transition at high-pressure conditions in slurry bubble columns is still not fully understood, and further studies are needed to examine the effect of solids concentration on the transition velocity, to develop an accurate correlation, and to explore the transition mechanism.

In general, the pressure effect on the flow regime transition is a result of the variation in bubble characteristics, such as bubble size and bubble size distribution. The bubble size and distribution are closely associated with factors such as initial bubble size, bubble coalescence rates and bubble breakup rates. Under high-pressure conditions, bubble coalescence is suppressed and bubble breakup is enhanced. Also, the distributor tends to generate smaller bubbles. All these factors contribute to small bubble sizes and narrow bubble size distributions and, consequently, delay the flow regime transition in high-pressure bubble columns and slurry bubble columns.

\subsection{Overall gas holdup and hydrodynamic similarity}

Gas holdup is a key parameter to characterize the macroscopic hydrodynamics of slurry bubble column systems. The gas holdup depends on gas and liquid velocities, gas distributor design, column geometry (diameter and height), physical properties of the gas and liquid, particle concentration, and physical properties of the particles. The gas holdup generally increases with gas velocity, with a larger rate of increase in the dispersed bubble regime than in the churn-turbulent regime. Such distributors as perforated plate, nozzle injector, and sparger affect the gas holdup significantly only at low gas velocities (Lin et al., 1999a). Lin et al. (1999a) also showed that the fluid dynamic behavior of gas and liquid in the plenum region is complex. They observed that the liquid flow in the bottom plenum to the bulk phase of the column through a perforated plate occurs via the liquid entrainment mechanism, i.e. turbulent gas bubbles and gas circulation in the gas layer entrain liquid from the liquid layer to the distributor as shown in Fig. 16. The gas and liquid flow patterns given in Fig. 16 may characterize, for example, an ebullated bed reactor for resid hydrotreating. In bubble columns, the effect of column size on gas holdup is negligible when the column diameter is larger than 0.1 to $0.15 \mathrm{~m}$ (Shah et al., 1982). The influence of the column height is insignificant if the height is above 1 to 3 meters and the ratio of the column height to the diameter is larger than 5 (Kastaneck et al., 1984). Gas holdup decreases as liquid viscosity and/or gas-liquid surface tension increase; however, the effect of liquid density is not clear. The addition of particles into a bubble 
column leads to a larger bubble size and thus a decreased gas holdup, especially when the particle concentration is low. The particle size effect on the gas holdup can be ignored in the particle size range of 44 to $254 \mu \mathrm{m}$.

Numerous studies have been conducted to investigate the effect of pressure on the gas holdup of bubble columns (Deckwer et al., 1980; Tarmy et al., 1984; Idogawa et al., 1986; Kojima et al., 1991; Wilkinson et al., 1992; Reilly et al., 1994; Jiang et al., 1995; Inga, 1997; Letzel et al., 1997; Lin et al., 1998) and three-phase fluidized beds (Luo et al., 1997a). Further, empirical correlations have been proposed for gas holdup in bubble columns operated at elevated pressure and temperature (Wilkinson et al., 1992; Reilly et al., 1994). It is commonly accepted that elevated pressures lead to a higher gas holdup in both bubble columns and three-phase fluidized beds except in those systems which are operated with porous plate distributors and at low gas velocities. The increased gas holdup is directly related to the smaller bubble size and, to a lesser extent, to the slower bubble rise velocity at higher pressures (Luo et al., 1997b). Figure 17 shows bubbles emerging from the three-phase fluidized bed of Paratherm NF heat transfer fluid and 2.1-mm glass beads over a wide range of operating conditions. As shown in the figure, bubble size is drastically reduced as pressure increases. The most fundamental reason for the bubble size reduction can be attributed to the variation in physical properties of the gas and liquid with pressure.

A significant pressure effect on the gas holdup should exist in slurry bubble columns; however, little is reported concerning such an effect. Deckwer et al. (1980) found little effect of pressure on gas holdup in a Fischer-Tropsch slurry bubble column with a porous plate distributor $(P=0.4$ to 1.1 $\mathrm{MPa} ; T=143$ to $260^{\circ} \mathrm{C} ; U_{g}=0$ to $3.5 \mathrm{~cm} / \mathrm{s}$ ). The experimental data of Kojima et al. (1991) indicated that the gas holdup increases with pressure; but no pressure effect was observed at the 30 $\mathrm{wt} \%$ solids concentration ( $P=0.1$ to $1.1 \mathrm{MPa} ; U_{g}=1.7$ to $9 \mathrm{~cm} / \mathrm{s}$; single orifice distributor). Inga (1997) measured the gas holdup in slurry bubble columns at pressures up to $0.72 \mathrm{MPa}$ and a significant pressure effect was observed. In general, no viable model is available to predict the gas holdup in high-pressure slurry bubble columns. The gas holdup behavior in high-pressure slurry bubble columns is not well understood, especially at high gas velocities.

The dynamic gas disengagement technique, first applied in bubble columns by Sriram and Mann (1977), is utilized to measure the gas holdup in a slurry bubble column under wide operating conditions (Lee et al., 1998). The results obtained with this technique for high-pressure systems are given in Luo et al. (1998a). Elevated pressures lead to higher gas holdups in a slurry bubble column. 
The presence of particles reduces the gas holdup at both ambient and elevated pressures as shown in Fig. 18. An empirical correlation is obtained to estimate the gas holdup in high-pressure slurry bubble columns as

$$
\frac{\varepsilon_{g}}{1-\varepsilon_{g}}=\frac{2.9\left(\frac{U_{g}^{4} \rho_{g}}{\sigma g}\right)^{\alpha}\left(\frac{\rho_{g}}{\rho_{m}}\right)^{\beta}}{\left[\cosh \left(M o_{m}^{0.054}\right)\right]^{4.1}}
$$

where $M o_{m}$ is the modified Morton number for the slurry phase, $g\left(\rho_{m}-\rho_{g}\right)\left(\xi \mu_{l}\right)^{4} / \rho_{m}^{2} \sigma^{3}$, and

$$
\begin{gathered}
\alpha=0.21 M o_{m}^{0.0079} \\
\beta=0.096 M o_{m}^{-0.011} .
\end{gathered}
$$

A correction factor $\xi$ accounts for the effect of particles on the slurry viscosity:

$$
\ln \xi=4.6 \varepsilon_{s}\left\{5.7 \varepsilon_{s}^{0.58} \sinh \left[-0.71 \exp \left(-5.8 \varepsilon_{s}\right) \ln (M o)^{0.22}\right]+1\right\} .
$$

Table 3 lists the various experimental systems and their corresponding references used to obtain the correlation. The average error of the predictions is $13 \%$ for both the slurry and gas-liquid systems and the maximum error is 53\%. The applicable ranges of the correlation are summarized in Table 4. The physical meaning of the dimensionless group of $U_{g}^{4} \rho_{g} / \sigma g$ in Eq. (34) can be shown by substituting Eq. (31) into the group:

$$
\frac{U_{g}^{4} \rho_{g}}{\sigma g} \propto\left(\frac{U_{g}}{u_{\max }}\right)^{4}
$$

Clearly, the dimensionless group represents the contribution of large bubbles to the overall gas holdup, which is the major reason why the correlation can cover such wide ranges of experimental conditions.

For high-pressure bubble columns and slurry bubble columns operated under the wide range of conditions outlined in Table 3, hydrodynamic similarity requires the following dimensionless groups to be the same: $U_{g} / u_{\max }, M o_{m}$, and $\rho_{g} / \rho_{m}$. To simulate the hydrodynamics of industrial reactors, cold models can be used and milder pressure and temperature conditions can be chosen, as long as the three groups are similar to those in the industrial reactor. The similarity rule needs to be tested in industrial reactors. 


\subsection{Bubble size distribution and dominance of large bubbles}

The bubble size can be measured by photographic or probe techniques. In multibubble systems, a mean bubble size is usually used to describe the system. The mean bubble size is commonly expressed through the Sauter, or volume-surface, mean. For a group of bubbles with measured diameters, the Sauter mean is

$$
d_{v s}=\frac{\sum n_{i} d_{b i}^{3}}{\sum n_{i} d_{b i}^{2}}
$$

where $n_{i}$ is the number of bubbles in the class $i$ with its volume equivalent size $d_{b i}$.

Some studies have been conducted to investigate pressure or gas density effects on mean bubble size and bubble size distribution in bubble columns (Idogawa et al., 1986, 1987; Jiang et al., 1995; Soong et al., 1997; Lin et al., 1998) as well as in three-phase fluidized beds (Jiang et al., 1992, 1997). According to these experimental studies, pressure has a significant effect on mean bubble diameter. The mean bubble diameter decreases with increasing pressure; however, above a certain pressure, the bubble size reduction is not significant. The effect of pressure on the mean bubble size is due to the change of bubble size distribution with pressure. At atmospheric pressure, the bubble size distribution is broad, while under high pressure, the bubble size distribution becomes narrower and is in smaller size ranges as shown in Fig. 19 (Luo et al., 1998a). At ambient conditions and $U_{g}=38.5$ $\mathrm{cm} / \mathrm{s}$, the slurry bubble column is in the slugging regime with the maximum bubble size of $7.2 \mathrm{~cm}$, approximately. At $P=5.6 \mathrm{MPa}$, bubble size is much smaller and slugs are not observed even at $U_{g}$ $=37.4 \mathrm{~cm} / \mathrm{s}$. According to the literature, bubble size is affected by bubble formation at gas distributor, bubble coalescence and bubble breakup. When the pressure is increased, the bubble size at the distributor is reduced (Luo et al., 1998c), bubble coalescence is suppressed (Jiang et al., 1995), and large bubbles tend to breakup, i.e. the maximum stable bubble size is reduced (Luo et al., 1998a). The combination of these three factors causes the decrease of mean bubble size with increasing pressure.

The bubble size distribution can normally be approximated by a log-normal distribution with its upper limit at the maximum stable bubble size. The contribution of bubbles of different sizes can be examined by analyzing the relationship between overall gas holdup and bubble size distribution. In slurry bubble columns, the gas holdup can be related to the superficial gas velocity, $U_{g}$, and the average bubble rise velocity, $\bar{u}_{b}$, (based on bubble volume) by a simple equation:

$$
U_{g}=\varepsilon_{g} \bar{u}_{b} .
$$


When the distributions of bubble size and bubble rise velocity are taken into account, $\bar{u}_{b}$ can be expressed as

$$
\bar{u}_{b}=\frac{\int_{d_{b, \text { min }}}^{d_{b, \text { max }}} V_{b}\left(d_{b}\right) f\left(d_{b}\right) u_{b}\left(d_{b}\right) \mathrm{d} d_{b}}{\int_{d_{b, \text { min }}}^{d_{b, \text { max }}} V_{b}\left(d_{b}\right) f\left(d_{b}\right) \mathrm{d} d_{b}} .
$$

The outcome of Equation. (40) and the gas holdup strongly depend on the existence of large bubbles, because of the large volume and high rise velocity of such large bubbles. An experimental study by Lee et al. (1998) revealed that, in the coalesced bubble regime, more than $70 \%$ of the small bubbles are entrained by the wakes of large bubbles and consequently have a velocity close to that of large bubbles. It is clear that the large bubbles have a dominant effect on the overall hydrodynamics of slurry bubble columns due to their large volume, their high rise velocity, and the wakes associated with the large bubbles.

\subsection{Heat transfer characteristics}

Studies reported in the literature for heat transfer characteristics in slurry bubble columns (Saxena et al., 1990; Li and Prakash, 1997) have been limited to ambient conditions. Little has been reported for high-pressure conditions. Since heat transfer behavior is closely associated with macroscopic flow structures and microscopic flow characteristics, a variation in pressure, which alters the physical properties of the gas and liquid, and also affects the hydrodynamics, would yield a complex effect on heat transfer behavior in the system. Previous studies on heat transfer in three-phase fluidized beds with liquids of different viscosity indicate that liquid viscosity has a negative effect on heat transfer (Kato et al., 1981; Kang et al., 1985). Since liquid viscosity increases with pressure, pressure would have a negative effect on heat transfer. Other physical properties of liquid, which are less affected by pressure, include liquid density, $\rho_{l}$, liquid thermal conductivity, $k_{l}$, and liquid heat capacity, $C_{p l}$ (Reid et al., 1977).

Studies on instantaneous heat transfer in liquids and liquid-solid systems that involve the injection of single bubbles revealed the importance of bubble wakes to heat transfer behavior (Kumar et al., 1992). The heat transfer enhancements by bubbles increases with bubble size due to the increased wake size and wake vortical intensity. When the pressure increases, the bubble size decreases, and hence the wake contribution to the heat transfer by single bubbles is reduced. In chain bubbling systems, Kumar and Fan (1994) reported that the time-averaged heat transfer coefficient increases with bubbling frequency due to the intense bubble-wake, bubble-bubble, and bubble-surface 
interactions. The effect of pressure on heat transfer due to the variations in liquid properties and hydrodynamic parameters is summarized in Table 5. The overall effect of pressure on heat transfer behavior depends on the outcome of the counteracting effects of each individual factor.

Deckwer et al. (1980) measured the heat transfer coefficient from an immersed heat source to the surrounding gas-liquid or gas-liquid-solid systems under conditions which prevail the FischerTropsch slurry process $\left(P=0.1\right.$ to $1.0 \mathrm{MPa} ; T=250$ to $300^{\circ} \mathrm{C} ; 16 \mathrm{wt} \%$ of $5 \mu \mathrm{m}$ particles $)$. Based on the surface renewal model and Kolmogoroff's theory of isotropic turbulence, a correlation was obtained to predict the heat transfer coefficient in slurry bubble columns:

$$
S t=0.1\left(\operatorname{Re} \operatorname{Fr} \operatorname{Pr}^{2}\right)^{-0.25} \text {. }
$$

In the model, the liquid-solid suspension was considered as a homogeneous phase, and consequently, the estimation scheme of the physical properties of the suspension from the individual phase was required.

Luo et al. (1997a) studied the heat transfer behavior in a three-phase fluidized bed over a pressure range of 0.1 to $15.6 \mathrm{MPa}$. Two types of glass beads, 2.1 and $3 \mathrm{~mm}$ in diameter, were used as the solid phase. The effects of gas velocity and pressure on the heat transfer coefficient are shown in Fig. 20. With an increase in pressure, the heat transfer coefficient increases, reaches a maximum at pressures of 6 to $8 \mathrm{MPa}$, and then decreases. An empirical equation is proposed to correlate the experimental data in their study:

$$
h=h^{\prime} \varepsilon_{g}^{0.45}\left(\frac{0.396}{U_{g}^{0.45}}+\frac{0.6768}{u_{p t, 0}}\right)
$$

where $h^{\prime}$ is the heat transfer coefficient of a liquid-solid fluidized bed with the same solids holdup, and $u_{p t, 0}$ is the particle terminal velocity in the fluidizing liquid at ambient pressure. In Eq. (42), the units for $U_{g}$ and $u_{p t, 0}$ are in $\mathrm{m} / \mathrm{s}$. The heat transfer coefficient, $h$, can be calculated by the correlation given below (Richardson et al., 1976):

$$
N u^{\prime}=0.67 \operatorname{Re}^{0.62} \operatorname{Pr}^{0.33} \frac{\varepsilon_{s}{ }^{0.38}}{1-\varepsilon_{s}} .
$$

The average deviation of the prediction from the experimental data is within $\pm 10 \%$.

Yang et al. (1998) studied heat transfer between an immersed solid surface and bulk fluids in a slurry bubble column at pressures of 0.1 to $6.3 \mathrm{MPa}$ and temperatures of 35 to $81^{\circ} \mathrm{C}$. Glass beads of $50 \mu \mathrm{m}$ in diameter are used as the solid phase. The solids concentrations are varied up to $35 \mathrm{vol} \%$, while the superficial gas velocities are varied up to $20 \mathrm{~cm} / \mathrm{s}$. The pressure effect on the heat transfer coefficient is shown in Fig. 21. It is found that pressure has a significant effect on the heat transfer 
characteristics in a slurry bubble column. The heat transfer coefficient decreases appreciably with increasing pressure except under very high pressures. The variation of the heat transfer coefficient with pressure is attributed to the counteracting effects of the variations of liquid viscosity, bubble size and bubbling frequency with pressure. When pressure increases, bubble size decreases; however, the bubbling frequency increases, which augments the rate of heat transfer (Kumar and Fan, 1994). The counteracting effects of the above two factors give rise to the overall effect of pressure on the heat transfer rate. In a slurry bubble column, pressure reduces bubble size significantly at pressures lower than $4 \mathrm{MPa}$, which results in the decrease of heat transfer coefficient. When the pressure is further increased, the bubble size reduction is relatively smaller, and the increase in bubbling frequency contributes to an increase in the heat transfer coefficient. However, in a three-phase fluidized bed, due to the large particle size, the bubble size reduction becomes a less important factor in affecting the heat transfer coefficient, and the heat transfer coefficient increases with the increase of bubbling frequency.

A consecutive film and surface renewal model originally developed by Wasan and Ahluwalia (1969) may be used to analyze the heat transfer behavior. The model assumes that a thin liquid film with a thickness of $\delta$ exists surrounding the heating surface; and liquid elements are forced to contact the outer surface of the film, due to the passage of bubbles. The liquid elements contact the film for a short time, $t_{c}$, and then, are replaced by fresh liquid elements. The heat is transferred to the bulk liquid through conduction by the liquid film and unsteady state conduction by the liquid elements. The heat transfer coefficient is expressed in terms of the physical properties of the liquid, the film thickness, and the contact time of the liquid elements (Wasan and Ahluwalia, 1969):

$$
h=\frac{2 k_{l}}{\sqrt{\pi \alpha t_{c}}}+\frac{k_{l} \delta}{\alpha t_{c}}\left[e^{\alpha t_{c} / \delta^{2}}\left(1-\operatorname{erf} \frac{\sqrt{\alpha t_{c}}}{\delta}\right)-1\right]
$$

Based on Eq. (44), the heat transfer coefficient is a function of film thickness and contact time between the liquid element and film. The order of magnitude of the film thickness may be estimated by (Kumar and Fan, 1994)

$$
\delta=\frac{6.14 L}{\operatorname{Re}_{m}^{3 / 4}} \operatorname{Pr}^{-1 / 3}
$$

where $R e_{m}$ is equal to $\rho_{m} L u_{b} / \mu_{m}$. Assuming that the element contact time is equal to the bubble contact time with the film, the contact time can be estimated from (Kumar and Fan, 1994)

$$
t_{c}=\frac{L}{u_{b}}
$$


where $u_{b}$ is the actual bubble rise velocity in a stream of bubbles. By considering the pressure effects on the physical properties of liquid and bubble characteristics, such as bubble size and bubble rise velocity, this model may be used to analyze the heat transfer behavior in a high-pressure system.

\section{CONCLUDING REMARKS}

Experimental results show the rise velocity of single bubbles in liquids and liquid-solid suspensions decreases with an increase in pressure and with a decrease in temperature. This decrease, combined with the pressure effect of reducing the bubble size, contributes to high gas holdups observed at high pressures. The bubble rise velocity in liquids and liquid-solid suspensions with low solids holdups can be reasonably estimated by use of the predictive equation available for ambient conditions, if the in-situ physical properties of the gas and liquid are used. Significant reduction in the rise velocity occurs at high solids holdups, especially for high liquid viscosity. The extent of reduction can be examined in terms of an increase in the apparent suspension viscosity by applying the homogeneous, Newtonian analogy. Along with the experimental results, discrete-phase simulations of a single bubble rising in liquid-solid suspensions at ambient conditions and in liquids at an elevated pressure are presented. A mechanistic model is described which accounts for the initial bubble size from a single orifice in liquid-solid suspensions. The mechanistic analysis indicates that the heterogeneous characteristics of liquid-solid suspensions can be satisfactorily accounted for by considering the particle-bubble collision behavior. The proposed mechanistic model successfully predicts the initial bubble size from a single orifice in high-pressure liquid-solid suspensions. The mechanism for bubble breakup at high pressures is illustrated by considering the bubble instability induced by internal gas circulation inside a bubble, and an analytical expression is obtained to quantify the maximum stable bubble size. Theoretical and experimental examinations on the roles of bubbles of different sizes indicate the important role that large bubbles play in dictating the macroscopic hydrodynamics of slurry bubble columns. An empirical correlation is provided to predict the gas holdup in slurry bubble columns over a wide range of conditions. A similarity rule is revealed for the overall hydrodynamics of high-pressure slurry bubble columns, which takes into account the operating conditions, the maximum stable bubble size, and the physical properties of the gas, liquid, and solids. A consecutive film and surface renewal model is used to explore the heat transfer characteristics in high-pressure three-phase fluidized beds and slurry bubble columns. 


\section{ACKNOWLEDGEMENT}

This work was supported by the National Science Foundation Grant CTS-9528380, the U.S. Department of Energy Grant DEFC22-95 PC 95051 with Cooperative Agreement with Air Products and Chemicals, Inc., and the Ohio State University / Industry Consortium Program on Fluidization and Multiphase Flow.

\section{NOTATION}

\begin{tabular}{|c|c|}
\hline$a$ & half $x$-axis length in Fig. 12 \\
\hline$C$ & constant in Eq. (31) \\
\hline$C_{D}$ & drag coefficient \\
\hline$C_{p l}$ & liquid heat capacity \\
\hline$c$ & parameter in Eq. (1) reflecting surface tension effect \\
\hline$D_{c}$ & column diameter \\
\hline$D_{\max }$ & maximum stable bubble size \\
\hline$D_{o}$ & orifice diameter \\
\hline$d_{b}$ & volume equivalent bubble diameter \\
\hline$d_{b}^{\prime}$ & dimensionless bubble diameter \\
\hline$d_{p}$ & particle diameter \\
\hline$d_{v s}$ & Sauter mean bubble diameter \\
\hline $\mathrm{E}\left(\sqrt{1-\alpha^{2}}\right)$ & complete second kind Elliptic integral \\
\hline Eo & Eötvös number \\
\hline$e$ & restitution coefficient \\
\hline $\boldsymbol{F}_{A M}$ & added mass force \\
\hline$F_{B}$ & buoyancy force \\
\hline $\boldsymbol{F}_{\text {Basset }}$ & Basset force \\
\hline$F_{c}$ & particle-bubble collision force \\
\hline $\boldsymbol{F}_{D}$ & liquid drag force \\
\hline $\boldsymbol{F}_{G / B}$ & gravity/buoyancy force \\
\hline$F_{I, g}$ & bubble inertial force \\
\hline
\end{tabular}




\begin{tabular}{|c|c|}
\hline$F_{I, m}$ & liquid-solid suspension inertial force \\
\hline$F_{M}$ & gas momentum force \\
\hline $\boldsymbol{F}_{\text {Magnus }}$ & Magnus force \\
\hline$F_{\text {total }}$ & total force \\
\hline$F_{x}$ & $\mathrm{x}$-component of centrifugal force induced by internal circulation \\
\hline$F_{\sigma}$ & surface tension force \\
\hline$F r$ & Froude number \\
\hline$f_{b}$ & volumetric body force \\
\hline$f_{s v}$ & volume force within free surface \\
\hline$f\left(d_{b}\right)$ & probability density function of bubble size \\
\hline$g$ & gravitational acceleration \\
\hline$H$ & column height \\
\hline$h$ & time-averaged heat transfer coefficient \\
\hline$h^{\prime}$ & heat transfer coefficient in liquid-solid fluidized beds \\
\hline$K$ & proportionality constant defined by Eq. (9a) \\
\hline$K_{b}$ & parameter in Eq. (1) reflecting viscous nature of surrounding medium \\
\hline$K_{b 0}$ & proportionality constant defined by Eq. (3d) \\
\hline$k_{l}$ & liquid thermal conductivity \\
\hline$k_{o}$ & orifice constant \\
\hline$L$ & circumference of the ellipse \\
\hline & length of the heat transfer probe \\
\hline$l$ & thickness of the liquid film between two coalescing bubbles \\
\hline Mo & Morton number \\
\hline$M o_{m}$ & modified Morton number based on slurry properties \\
\hline$m_{p}$ & particle mass \\
\hline$N c$ & dimensionless capacitance number \\
\hline$N u^{\prime}$ & Nusselt number in liquid-solid fluidized beds \\
\hline$n$ & parameter in Eq. (1) reflecting system purity \\
\hline$n_{i}$ & number of bubbles \\
\hline$P$ & pressure \\
\hline$P_{b}$ & pressure in the bubble \\
\hline
\end{tabular}




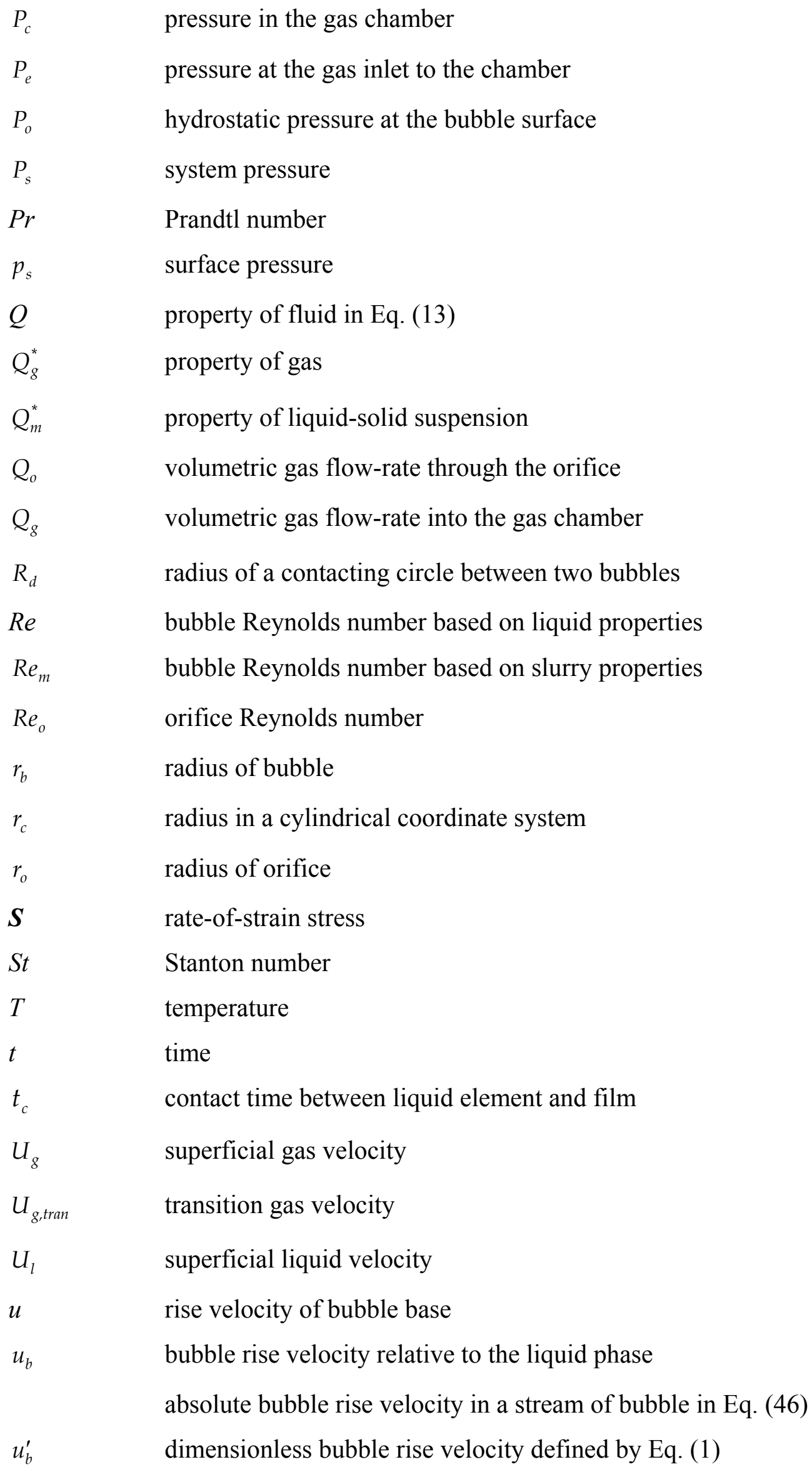




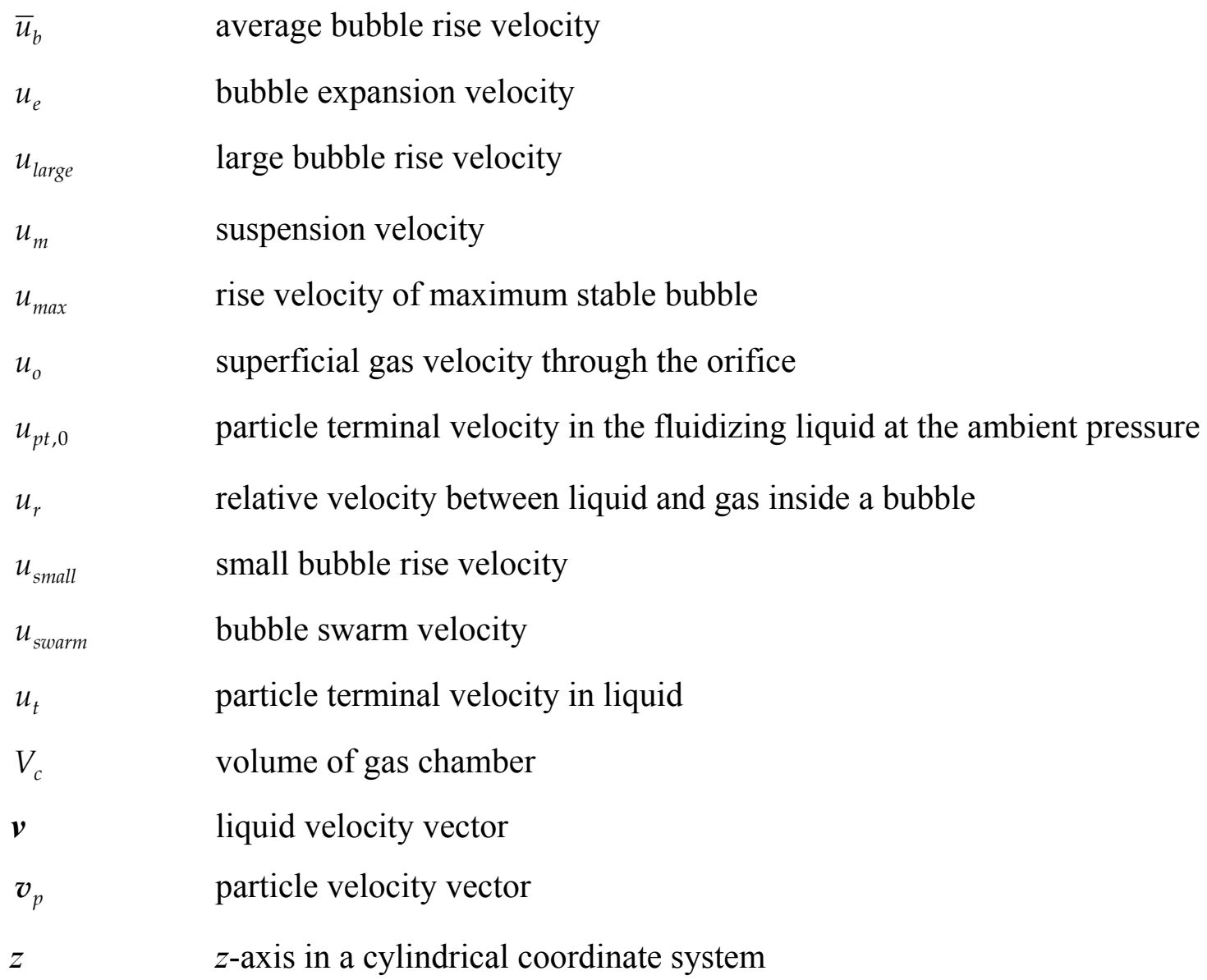




$\begin{array}{ll}\kappa & \text { contact angle } \\ \lambda_{c} & \text { free surface curvature } \\ \mu & \text { critical wavelength } \\ \mu_{g} & \text { coefficient of dynamic viscosity } \\ \mu_{l} & \text { gas viscosity } \\ \mu_{m} & \text { liquid viscosity } \\ \rho_{g} & \text { (effective) viscosity of liquid-solid suspension } \\ \rho_{l} & \text { gas density } \\ \rho_{m} & \text { liquid density } \\ \rho_{s} & \text { density of liquid-solid suspension } \\ \sigma & \text { solids density } \\ \tau & \text { surface tension } \\ \xi & \text { viscous stress tensor } \\ \zeta & \text { correction factor defined by Eq. (36) } \\ & \text { coefficient in Eq. (20c) }\end{array}$




\section{REFERENCES}

Akita, K. and F. Yoshida, 1973. Gas holdup and volumetric mass transfer coefficient in bubble columns. Ind. Eng. Chem. Process Des. Develop., 12, 76.

Allen, M. P. and D. J. Tildesley, 1987. Computer Simulation of Liquids, Clarendon Press, Oxford.

Azbel, D., 1981. Two-Phase Flows in Chemical Engineering, Cambridge Univ. Press, Cambridge, UK.

Bach, H. F. and T. Pilhofer, 1978. Variation of gas hold-up in bubble columns with physical properties of liquids and operating parameters of columns. Ger. Chem. Eng., 1, 270.

Batchelor, G. K., 1987. The stability of a large gas bubble rising through liquid. J. Fluid Mech., 184, 399.

Batchelor, G. K., 1988. A new theory of the instability of a uniform fluidized bed. J. Fluid Mech., $193,75$.

Bavarian, F., and L.-S. Fan, 1991a. Mechanisms of hydraulic transport of a packed bed at the startup of a three-phase fluidized bed. Chem. Eng. Sci., 46, 3081.

Bavarian, F., and L.-S. Fan, 1991b. Hydraulic transport of a packed bed during the start-up of a three-phase fluidized bed with large gas holdups. Ind. Eng. Chem. Res., 30, 408.

Bellman, R. and R. H. Pennington, 1954. Effect of surface tension and viscosity on Taylor instability. Q. Appl. Math., 51, 151.

Bhaga, D. and M. E. Weber, 1981. Bubbles in viscous liquids: shapes, wakes, and velocities. J. Fluid Mech., 105, 61.

Brackbill, J. U., D. B. Kothe, and C. Zemach, 1992. A continuum method for modeling surface tension. J. Comp. Phys., 100, 335.

Chaudhari, R. V. and H. Hoffmann, 1994. Coalescence of gas bubbles in liquids. Rev. Chem. Eng., 10, 131.

Chen, Y.-M. and L.-S. Fan, 1988. On the criteria of Rayleigh-Taylor instability at a curved interface by a local force balance. Unpublished work.

Chen, Y.-M. and L.-S. Fan, 1989. Bubble breakage due to particle collision in a liquid medium. Chem. Eng. Sci., 44, 2762.

Clark, K. N., 1990. The effect of high pressure and temperature on phase distributions in a bubble column. Chem. Eng. Sci., 45, 2301.

Clift, R., J. R. Grace, and M. E. Weber, 1978. Bubbles, Drops, and Particles. Academic Press, New York. 
Darton, R. C. and D. Harrison, 1974. The rise of single gas bubbles in liquid fluidized beds. Trans. Instn Chem. Engrs, 52, 301.

Davenport, W. G., F. D. Richardson, and A. V. Bradshaw, 1967. Spherical cap bubbles in low density liquids. Chem. Eng. Sci., 22, 1221.

Davies, R. M. and G. I. Taylor, 1950. The mechanics of large bubbles rising through extended liquids and through liquids in tubes. Proc. Roy. Soc. London, A200, 375.

Deckwer, W.-D., 1992. Bubble Column Reactors, John Wiley and Sons, Chichester, England.

Deckwer, W.-D., Y. Louisi, A. Zaidi, and M. Ralek, 1980. Hydrodynamic properties of the FischerTropsch slurry process. Ind. Eng. Chem. Process Des. Dev., 19, 699.

El-Temtamy, S. A. and N. Epstein, 1979. Contraction of expansion of three-phase fluidized beds containing fine/light solids. Can. J. Chem. Eng., 57, 520.

El-Temtamy, S. A. and N. Epstein, 1980. Rise velocities of large single two-dimensional and threedimensional gas bubbles in liquids and in liquid fluidized beds. Chem. Eng. J., 19, 153.

Fan, L.-S., 1989. Gas-Liquid-Solid Fluidization Engineering, Butterworths, Stoneham, MA.

Fan, L.-S., 1999. Moving packed bed phenomena in three-phase fluidization. Powder Technology, in press.

Fan, L.-S. and K. Tsuchiya, 1990. Bubble Wake Dynamics in Liquids and Liquid-Solid Suspensions. Butterworth-Heinemann, Stoneham, MA.

Fox, J. M., 1990. Fischer-Tropsch reactor selection. Catal. Lett., 7, 281.

Grace, J. R., T. Wairegi, and J. Brophy, 1978. Break-up of drops and bubbles in stagnant media. Can. J. Chem. Eng., 56, 3.

Grund, G., A. Schumpe, and W.-D. Deckwer, 1992. Gas-liquid mass transfer in a bubble column with organic liquids. Chem. Eng. Sci., 47, 3509.

Hill, M. J. M., 1894. On a spherical vortex. Phil. Trans. Roy. Soc. London, 185, 213.

Hinze, J. O., 1955. Fundamentals of the hydrodynamic mechanism of splitting in dispersion processes. AIChE J., 1, 289.

Hirt, C. W. and B. D. Nichols, 1981. Volume of fluid (VOF) method for the dynamics of free boundaries, J. Comp. Phys., 39, 201.

Hoefsloot, H. C. J. and R. Krishna, 1993. Influence of gas density on the stability of homogeneous flow in bubble columns. Ind. Eng. Chem. Res., 32, 747.

Hyndman, C. L., F. Larachi, and C. Guy, 1997. Understanding gas-phase hydrodynamics in bubble columns: A convective model based on kinetic theory. Chem. Eng. Sci., 52, 63. 
Idogawa, K., K. Ikeda, T. Fukuda, and S. Morooka, 1986. Behavior of bubbles of the air-water system in a column under high pressure. Int. Chem. Eng., 26, 468.

Idogawa, K., K. Ikeda, T. Fukuda, and S. Morooka, 1987. Formation and flow of gas bubbles in a single orifice or nozzle gas distributor. Chem. Eng. Comm., 59, 201.

Inga, J. R., 1997. Scaleup and scaledown of slurry reactors: a new methodology. Ph.D. thesis, University of Pittsburgh, PA.

Jager, B. and R. Espinoza, 1995. Advances in low temperature Fischer-Tropsch synthesis. Catal. Today, 23, 17.

Jean, R.-H. and L.-S. Fan, 1990. Rise velocity and gas-liquid mass transfer of a single large bubble in liquids and liquid-solid fluidized beds. Chem. Eng. Sci., 45, 1057.

Jiang, P., D. Arters, and L.-S. Fan, 1992. Pressure effects on the hydrodynamic behavior of gasliquid-solid fluidized beds. Ind. Eng. Chem. Res., 31, 2322.

Jiang, P., T.-J. Lin, X. Luo, and L.-S. Fan, 1995. Visualization of high pressure (21 MPa) bubble column: bubble characteristics. Chem. Eng. Res. \& Des., 73, 269.

Jiang, P., X. Luo, T.-J. Lin, and L.-S. Fan, 1997. High temperature and high pressure three-phase fluidization—bed expansion phenomena. Powder Technology, 90, 103.

Joshi, J. B. and A. M. Lali, 1984. Velocity-hold up relationship in multiphase contactors-a unified approach. Frontiers in Chem. Reaction Eng., 1, 314, Wiley Eastern, New York.

Kang, Y., I. S. Suh, and S. D. Kim, 1985. Heat transfer characteristics of three-phase fluidized beds. Chem. Eng. Comm., 34, 1.

Kastaneck, F., J. Zahradnik, J. Kratochvil, and J. Cermak, 1984. Modelling of large-scale bubble column reactors for non-ideal gas-liquid systems in Frontiers in Chemical Reaction Engineering, edited by L. K. Doraiswamy and R. A. Mashelkar, 1, 330, Whiley, Bombay, India.

Kato, Y., K. Uchida, and S. Morooka, 1981. Liquid holdup and heat transfer coefficient between bed and wall in liquid-solid and gas-liquid-solid fluidized beds. Powder Technology, 28, 173.

Kitscha, J. and G. Kocamustafaogullari, 1989. Breakup criteria for fluid particles. Int. J. Multiphase Flow, 15, 573.

Koide, K., A. Takazawa, and M. Komura, 1984. Gas Holdup and Volumetric Liquid-Phase Mass Transfer Coefficient in Solid-Suspended Bubble Columns. J. Chem. Engng. Japan, 17, 459.

Kojima, H., B. Okumura, and A. Nakamura, 1991. Effect of pressure on gas holdup in a bubble column and a slurry bubble column. J. Chem. Engng. Japan, 24, 115. 
Krishna, R., J. W. A. De Swart, D. E. Hennephof, J. Ellenberger, and C. J. Hoefsloot, 1994. Influence of increased gas density on hydrodynamics of bubble-column reactors. AIChE J., $\mathbf{4 0}$, 112.

Krishna, R., P. M. Wilkinson, and L. L. Van Dierendonck, 1991. A model for gas holdup in bubble columns incorporating the influence of gas density on flow regime transitions. Chem. Eng. Sci., 46, 2491.

Kumar, R. and N. R. Kuloor, 1970. The formation of bubbles and drops. Advances in Chemical Engineering, 8, 255.

Kumar, S., K. Kusakabe, K. Raghunathan, and L.-S. Fan, 1992. Mechanism of heat transfer in bubbly liquid and liquid-solid systems: single bubble injection. AIChE J., 38, 733.

Kumar, S. and L.-S. Fan, 1994. Heat-transfer characteristics in viscous gas-liquid and gas-liquidsolid systems. AIChE J., 40, 745.

Kupferberg, A. and G. J. Jameson, 1969. Bubble formation at a submerged orifice above a gas chamber of finite volume. Trans. Instn Chem. Engrs, 47, T241.

Lammers, J. H. and A. Biesheuvel, 1996. Concentration waves and the instability of bubbly flows. $J$. Fluid Mech., 328, 67.

LaNauze, R. D. and I. J. Harris, 1974. Gas bubble formation at elevated system pressures. Trans. Instn Chem. Engrs., 52, 337.

Letzel, H. M., J. C. Schouten, C. M. Van den Bleek, and R. Krishna, 1997. Influence of elevated pressure on the stability of bubbly flows. Chem. Eng. Sci., 52, 3733.

Letzel, M. H., J. C. Schouten, C. M. Van den Bleek, and R. Krishna, 1998. Effect of gas density on large-bubble holdup in bubble column reactors. AIChE J., 44, 2333.

Lee, D. J., X. Luo, and L.-S. Fan, 1998. Gas disengagement technique in a slurry bubble column operated in the coalesced bubble regime. Chem. Eng. Sci., in press.

Levich, V. G., 1962. Physiochemical Hydrodynamics, Prentice Hall, Englewood Cliffs, NJ.

Li, H., and A. Prakash, 1997. Heat transfer and hydrodynamics in a three-phase slurry bubble column. Ind. Eng. Chem. Res., 36, 4688.

Lin, J. N., S. K. Banerji, and H. Yasuda, 1994. Role of interfacial tension in the formation and the detachment of air bubbles. 1. A single hold on a horizontal plane immersed in water. Langmuir, 10, 936.

Lin, T.-J. and L.-S. Fan, 1997. Characteristics of high-pressure liquid-solid fluidization. AIChE J., 43, 45. 
Lin, T.-J., K. Tsuchiya, and L.-S. Fan, 1998. Bubble flow characteristics in bubble columns at elevated pressure and temperature. AIChE J., 44, 545.

Lin, T.-J., G. B. Bass, K. Tsuchiya, and L.-S. Fan, 1999a. Distributor effects on hydrodynamics of high pressure bubble columns. Chem. Eng. Sci., in review.

Lin, T.-J., K. Tsuchiya, and L.-S. Fan, 1999b. On the measurements of regime transition in high pressure bubble columns. Can. J. Chem. Eng., in press.

Liu, T.-J. and S. G. Bankoff, 1993. Structure of air-water bubbly flow in a vertical pipe - II. Void fraction, bubble velocity, and bubble size distribution. Int. J. Heat Mass Transfer, 36, 1061.

Luo, X., P. Jiang, and L.-S. Fan, 1997a. High pressure three-phase fluidization: hydrodynamics and heat transfer. AIChE J., 43, 2432.

Luo, X., J. Zhang, J., K. Tsuchiya, and L.-S. Fan, 1997b. On the rise velocity of bubbles in liquidsolid suspensions at elevated pressure and temperature. Chem. Eng. Sci., 52, 3693.

Luo, X., D. J. Lee, R. Lau, G. Q. Yang, and L.-S. Fan, 1998a. Maximum stable bubble size and gas holdup in high pressure slurry bubble columns. AIChE J., in press.

Luo, X., K. Tsuchiya, and L.-S. Fan, 1998b. Gas jetting and bubble formation in high pressure liquid-solid suspensions. In Fluidization $I X$, edited by L.-S. Fan and T. M. Knowlton, Engineering Foundation, NY, 637.

Luo, X., G. Q. Yang, D. J. Lee, and L.-S. Fan, 1998c. Single bubble formation in high pressure liquid-solid suspensions. Powder Technology, 100, 103.

Maneri, C. C., 1995. New look at wave analogy for prediction of bubble terminal velocities. $A I C h E$ J., 41, 481.

Massimilla, L., N. Majuri, and P. Signorini, 1959. Sull'assorbimento di gas in sistema: solido-liquido, fluidizzato. La Ricerca Scientifica, 29, 1934.

Massimilla, L., A. Solimando, and E. Squillace, 1961. Gas dispersion in solid-liquid fluidized beds. Brit. Chem. Eng., 6, 232.

Mendelson, H. D., 1967. The motion of an air bubble rising in water. AIChE J., 13, 250.

Mill, P. L., J. R. Turner, P. A. Ramachandran, and M. P. Dudukovic, 1996. The Fischer-Tropsch synthesis in slurry bubble column reactors: Analysis of reactor performance using the axial dispersion model. Three-phase sparged reactors, Edited by K.D.P. Nigam and A. Schumpe, Gordon and Breach Publishers, 339.

Milne-Thomson, L. M., 1955. Theoretical Hydrodynamics, 3rd ed., Macmillan and Co., London.

Miyahara, T. K., K. Tsuchiya, and L.-S. Fan, 1989. Mechanism of particle entrainment in a gasliquid-solid fluidized bed. AIChE J., 35, 1195. 
O’Dowd, W., D. N. Smith, J. A. Ruether, and S. C. Saxena, 1987. Gas and solids behavior in a baffled and unbaffled slurry bubble column. AIChE J., 33, 1959.

Ostergaard, K., 1964. Fluidization, Soc. Chem. Ind., London.

Oyevaar, M. H., 1989. Gas-liquid contacting at elevated pressures. Ph.D. thesis, Twente Univ., The Netherlands.

Peng, X. D., B. A. Toseland, and P. J. A. Tijm, 1998. Kinetic understanding of chemical synergy in $\mathrm{LPDME}^{\mathrm{TM}}$ process. Presented at the 1998 AIChE Annual Meeting, Nov. 15-20, Miami Beach, FL.

Petukhov, V. I. and V. A. Kolokol'tsev, 1965. Effect of liquid viscosity on droplet entrainment and volumetric air content. Therm. Eng., 12, 41.

Pinczewski, W. V., 1981. The formation and growth of bubbles at a submerged orifice. Chem. Eng. Sci., 36, 405.

Rados, N., 1999. Slurry bubble column hydrodynamics. D.Sc. proposal, Washington University, St. Louis.

Ramakrishnan, S., R. Kumar, and N. R. Kuloor, 1969. Studies in bubble formation - I: bubble formation under constant flow conditions. Chem. Eng. Sci., 24, 731.

Reid, R. C., J. M. Prausnitz, and T. K. Sherwood, 1977. The Properties of Gases and Liquids. McGraw-Hill, New York.

Reilly, I. G., D. S. Scott, T. J. W. de Bruijn, and D. MacIntyre, 1994. The role of gas phase momentum in determining gas holdup and hydrodynamic flow regimes in bubble column operations. Can. J. Chem. Eng., 72, 3.

Richardson, J. F., M. N. Roman, and K. J. Shakiri, 1976. Heat transfer from immersed surfaces in liquid fluidized beds. Chem. Eng. Sci., 31, 619.

Ruzicka, M. C., J. Drahos, J. Zahradnik, and N. H. Thomas, 1997. Intermittent transition from bubbling to jetting regime in gas-liquid two phase flows. Int. J. Multiphase Flow, 23, 671.

Saberian-Broudjenni, M., G. Wild, J. C. Charpentier, Y. Fortin, J. P. Euzen, and R. Patoux, 1984. Contribution à l'ètude hydrodynamique des rèacteurs à lit fludisè gaz-liquide-solide, Entropie, No. $120,30$.

Sagert, N. H. and M. J. Quinn, 1977. Influence of high-pressure gases on the stability of thin aqueous films. J. Colloid Int. Sci., 61, 279.

Sagert, N. H. and M. J. Quinn, 1978. Surface viscosities at high pressure gas-liquid interfaces. $J$. Colloid Int. Sci., 65, 415. 
Saxena, S. C., 1995. Bubble column reactors and Fischer-Tropsch synthesis. Catal. Rev.-Sci. Eng., $37,227$.

Saxena, S. C. and Z. D. Chen, 1994. Hydrodynamics and heat transfer of baffled and unbaffled slurry bubble columns. Reviews in Chem. Eng., 10, 193.

Saxena, S. C., N. S. Rao, and A. C. Saxena, 1990. Heat Transfer from a cylindrical probe immersed in a three-phase slurry bubble column. Chem. Eng. J., 44, 141.

Saxena, S. C., R. Vadivel, and A. C. Saxena, 1989. Gas holdup and heat transfer from immersed surfaces in two- and three-phase systems in bubble columns. Chem. Eng. Comm., 85, 63.

Schumpe, A. and G. Grund, 1986. The gas disengagement technique for studying gas holdup structure in bubble columns. Can. J. Chem. Eng., 64, 891.

Shnip, A. I., R. V. Kolhatkar, D. Swamy, and J. B. Joshi, 1992. Criteria for the transition from the homogeneous to the heterogeneous regime in two-dimensional bubble column reactors. Int. J. Multiphase Flow, 18, 705.

Shah, Y. T., B. G. Kelkar, S. P. Godbole, and W.-D. Deckwer, 1982. Design parameters estimations for bubble column reactors. AIChE J., 28, 353.

Shollenberger, K. A. and T. J. O’Hern, 1997. Characterization of slurry-phase flow in the Laporte alternative fuels development unit (AFDU) using differential pressure measurements. U.S. DOE Report, DE-FC22-95 PC 95051.

Soong, Y., F. W. Harke, I. K. Gamwo, R. R. Schehl, and M. F. Zarochak, 1997. Hydrodynamic study in a slurry-bubble-column reactor. Catalysis Today, 35, 427.

Sriram, K. and R. Mann, 1977. Dynamic gas disengagement: A new technique for assessing the behaviour of bubble columns. Chem. Eng. Sci., 32, 571.

Tarmy, B., M. Chang, C. Coulaloglou, and P. Ponzi, 1984. Hydrodynamic characteristics of three phase reactors. The Chemical Engineer, Oct., No. 407, 18.

Tomiyama, A., I. Kataoka, and T. Sakaguchi, 1995. Drag coefficients of bubbles (1st report, drag coefficients of a single bubble in a stagnant liquid). Nippon Kikai Gakkai Ronbunshu B Hen, 61(587), 2357.

Tsuchiya, K., A. Furumoto, L.-S. Fan, and J. Zhang, 1997. Suspension viscosity and bubble velocity in liquid-solid fluidized beds. Chem. Eng. Sci., 52, 3053.

Tsuchiya, K. and O. Nakanishi, 1992. Gas holdup behavior in a tall bubble column with perforated plate distributors. Chem. Eng. Sci., 47, 3347.

Tsuchiya, K., G.-H. Song, W.-T. Tang, and L.-S. Fan, 1992. Particle drift induced by a bubble in a liquid-solid fluidized bed with low-density particles, AIChE J., 38, 1847. 
Tsuge, H., Y. Nakajima, and K. Terasaka, 1992. Behavior of bubbles formed from a submerged orifice under high system pressure. Chem. Eng. Sci., 47, 3273.

Vrij, A., 1966. Possible mechanism for the spontaneous rupture of thin, free liquid films. Disc. Faraday Soc., 42, 23.

Wallis, G. B., 1969. One-Dimensional Two-Phase Flow, McGraw-Hill, New York.

Walter, J. F. and H. W. Blanch, 1986. Bubble break-Up in gas-liquid bioreactors: break-up in turbulent flows. Chem. Eng. J., 32, B7.

Wasan, D. T. and M. S. Ahluwalia, 1969. Consecutive film and surface renewal mechanism for heat and mass transfer from a wall. Chem. Eng. Sci., 24, 1535.

Wilkinson, P. M., A. P. Sper, and L. L. Van Dierendonck, 1992. Design parameters estimation for scale-up of high-pressure bubble columns. AIChE J., 38, 544.

Wilkinson, P. M. and L. L. Van Dierendonck, 1990. Pressure and gas density effects on bubble break-up and gas hold-up in bubble columns. Chem. Eng. Sci., 45, 2309.

Wilkinson, P. M. and L. L. Van Dierendonck , 1994. A theoretical model for the influence of gas properties and pressure on single-bubble formation at an orifice. Chem. Eng. Sci., 49, 1429.

Yang, G. Q., X. Luo, R. Lau, and L.-S. Fan, 1998. Heat transfer characteristics in a high pressure slurry bubble column. Presented at the 1998 AIChE Annual Meeting, paper 191b, Nov. 15-20, Miami Beach, FL.

Yang, G. Q., X. Luo, R. Lau, and L.-S. Fan, 1999. Single bubble formation in high pressure liquidsolid suspensions with pressure fluctuations in the gas chamber. Chem. Eng. Sci., in review.

Yoo, D.-H., H. Tsuge, K. Terasaka, and K. Mizutani, 1997. Behavior of bubble formation in suspended solution for an elevated pressure system. Chem. Eng. Sci., 52, 3701.

Yu, Y. H. and S. D. Kim, 1991. Bubble properties and local liquid velocity in the radial direction of cocurrent gas-liquid flow. Chem. Eng. Sci., 46, 313.

Zahradnik, J., M. Fialova, M. Ruzicka, J. Drahos, F. Kastanek, and N. H. Thomas, 1997. Duality of the gas-liquid flow regimes in bubble column reactors, Chem. Eng. Sci., 52, 3811.

Zhang, J., L.-S. Fan, C. Zhu, R. Pfeffer, and D. Qi, 1998a. Dynamic behavior of collinear collision of elastic spheres in viscous fluids, Advanced Technologies for Particle Processing, Vol. II, 44, Particle Technology Forum, AIChE; Proceedings of PTF Topical Conference at AIChE Annual Meeting, Nov. 15-20, Miami Beach, FL; Powder Technology, in press.

Zhang, J., Y. Li, and L.-S. Fan, 1998b. Numerical simulation of gas-liquid-solid fluidization systems using a combined CFD-DPM-VOF method: single bubble rise behavior, Advanced Technologies 
for Particle Processing, Vol. II, 509, Particle Technology Forum, AIChE; Proceedings of PTF Topical Conference at AIChE Annual Meeting, Nov. 15-20, Miami Beach, FL. 


\section{FIGURES}

Figure 1 Effect of pressure on terminal rise velocity of single bubbles and predicted values at (a) $27^{\circ} \mathrm{C}$ and (b) $78^{\circ} \mathrm{C}$.

Figure 2 Comparisons of measured and calculated $R e$ of single bubbles in Paratherm NF heat transfer fluid under varied pressure and temperature conditions. The Fan-Tsuchiya (1990) and Tomiyama et al. (1995) correlations are plotted ( - and -respectively) at regular intervals of Mo values. The Fan-Tsuchiya correlation at measured Mo values for comparison with measured Re-Eo data (-).

Figure 3 Effect of pressure on bubble rise velocity in a fluidized bed at (a) $26.5^{\circ} \mathrm{C}$ and (b) $87.5^{\circ} \mathrm{C}$. Solids holdups for + , open, and filled symbols are $0,0.384$, and 0.545 , respectively.

Figure 4 Effect of pressure on bubble rise velocity in a fluidized bed at (a) $26.5^{\circ} \mathrm{C}$ and (b) $87.5^{\circ} \mathrm{C}$. Solids holdups for + , open, and filled symbols are $0,0.381$, and 0.555 , respectively.

Figure 5 Simulation and experimental results of a bubble rising in a liquid-solid fluidized bed.

Figure 6 Simulation of a bubble emerging from a liquid-solid fluidized bed.

Figure 7 Simulation results of a single bubble rising at $P=19.4 \mathrm{MPa}$.

Figure 8 The balance of all the forces acting on a growing bubble.

Figure 9 Comparison between the experimental data and model predictions of initial bubble size in high-pressure liquid-solid suspensions. Lines and symbols represent the model predictions and experimental data, respectively.

Figure 10 Initial bubble size in liquid-solid suspensions as a function of pressure and gas velocity for bubble formation with pressure fluctuation in the gas chamber.

Figure 11 A series of photographs showing the bubbling-jetting transition at $P=4.24 \mathrm{MPa}$ and $T=$ $28^{\circ} \mathrm{C}$ for (a) $u_{o}=0.27 \mathrm{~m} / \mathrm{s}$ and $R e_{o}=1,075$; (b) $u_{o}=1.35 \mathrm{~m} / \mathrm{s}$ and $R e_{o}=5,321$; (c) $u_{0}$ $=2.23 \mathrm{~m} / \mathrm{s}$ and $R e_{o}=8,809 ;(\mathrm{d}) u_{o}=2.60 \mathrm{~m} / \mathrm{s}$ and $R e_{o}=10,243 ;(\mathrm{e}) u_{o}=3.99 \mathrm{~m} / \mathrm{s}$ and $R e_{o}=15,759 ;$ (f) $u_{o}=6.42 \mathrm{~m} / \mathrm{s}$ and $R e_{o}=25,355$.

Figure 12 Schematic of the internal circulation model for bubble breakup.

Figure 13 A sequence of bubble images showing the process of bubble breakup at $P=3.5 \mathrm{MPa}$.

Figure 14 Comparison of (a) the maximum stable bubble size and (b) the bubble velocities between the experimental data and the predictions by various models. 
Figure 15 Comparison of the regime transition velocity (a) in a bubble column (open symbols are obtained by standard deviation of pressure fluctuation and drift flux, and closed symbols are calculated by the Wilkinson et al. (1992) correlation) and (b) in a three-phase fluidized bed.

Figure 16 Visualization of liquid entrainment in the plenum by gas from the liquid layer through the gas layer to the perforated plate.

Figure 17 Visualization of bubbles emerging from the three-phase fluidized bed surface at (a) $P=$ $0.1 \mathrm{MPa}$, (b) $P=3.5 \mathrm{MPa}$, (a) $P=6.8 \mathrm{MPa}$, (a) $P=17.4 \mathrm{MPa}$.

Figure 18 Effect of (a) pressure and (b) solids concentration on the gas holdup in a slurry bubble column.

Figure 19 Bubble size distribution in a slurry bubble column at (a) $P=0.1 \mathrm{MPa}$ and (b) $P=5.6$ $\mathrm{MPa}$.

Figure 20 Effect of pressure on the heat transfer coefficient in a three-phase fluidized bed.

Figure 21 Heat transfer coefficient as a function of gas velocity at different pressures in a slurry bubble column. 


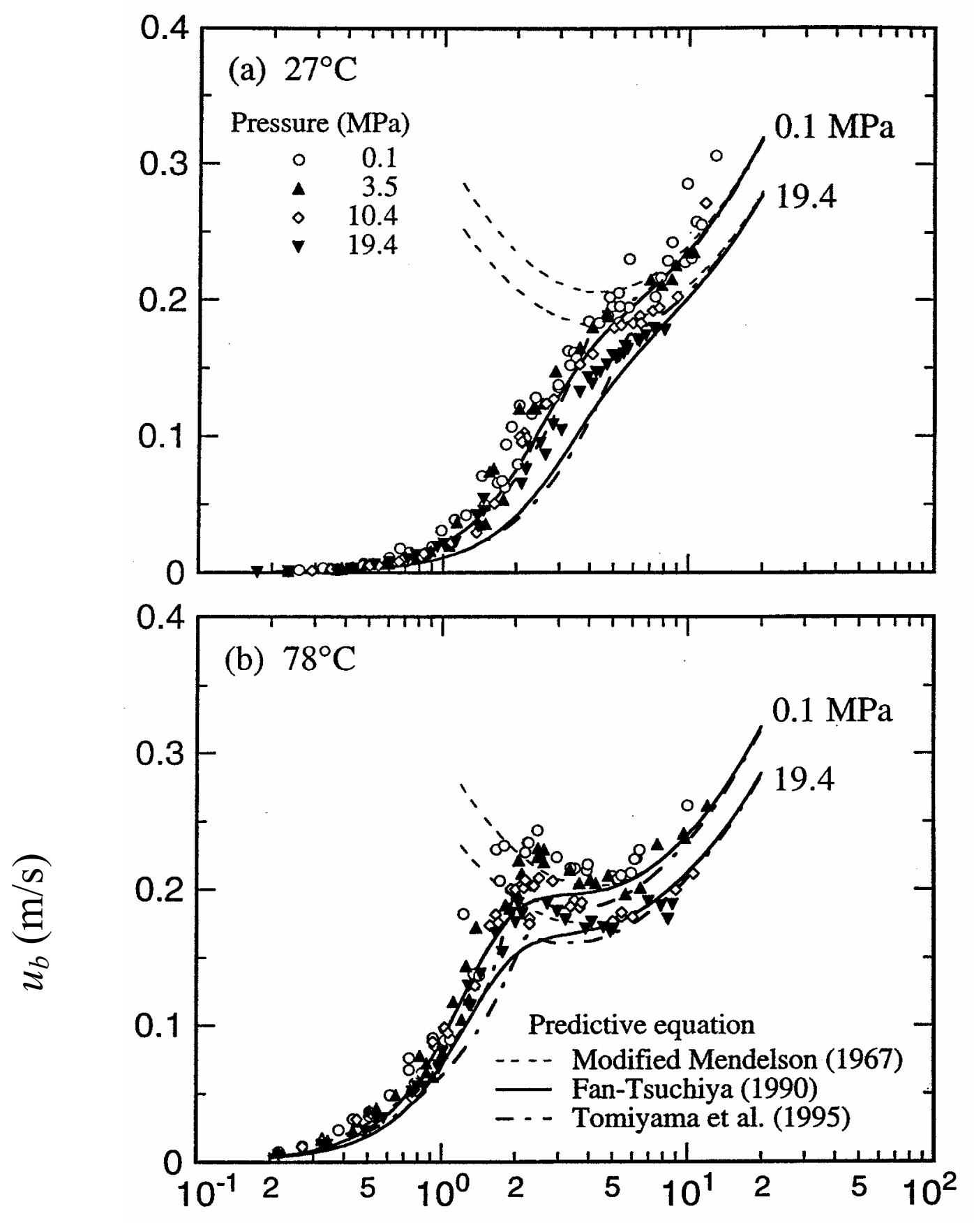

Figure 1 


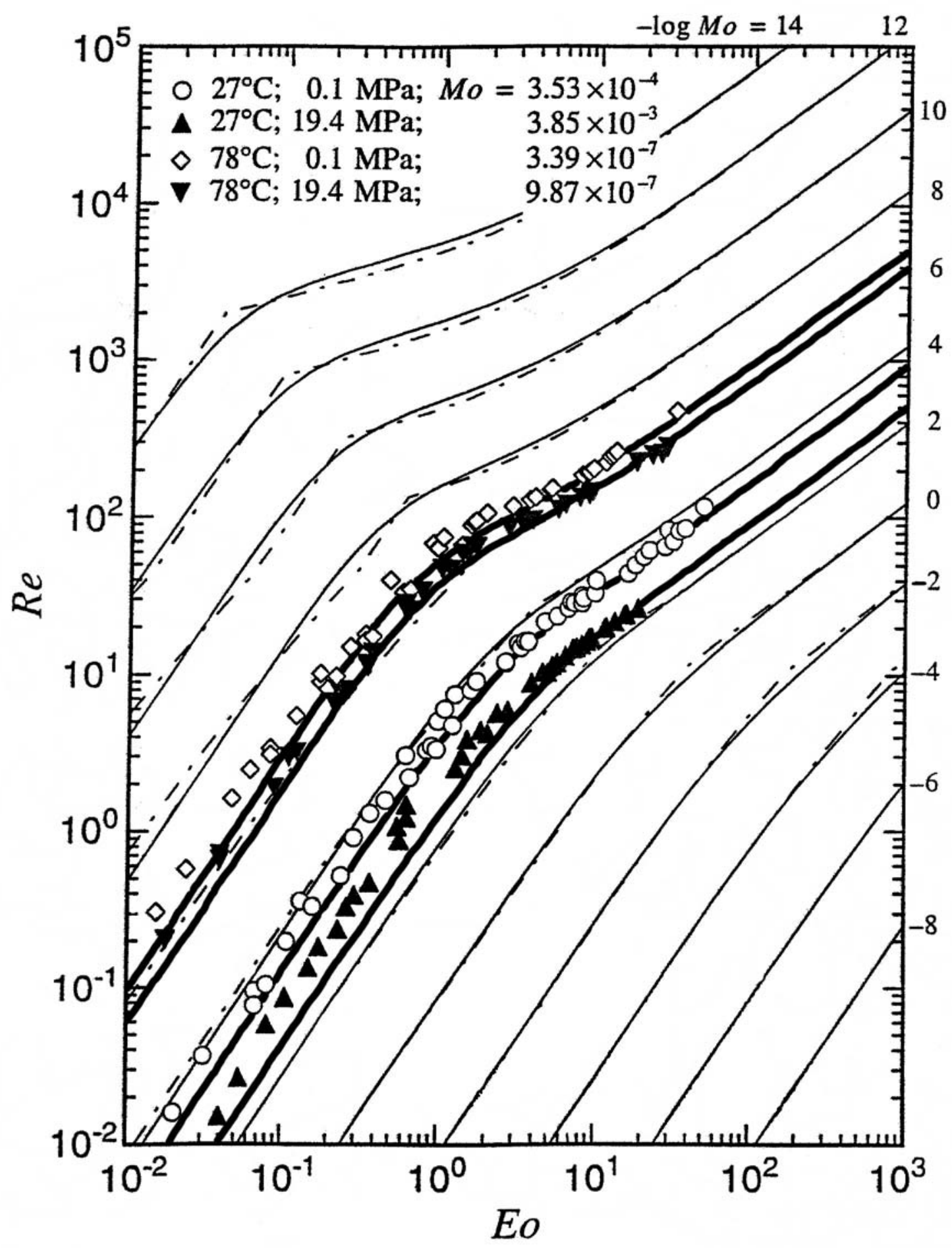

Figure 2 

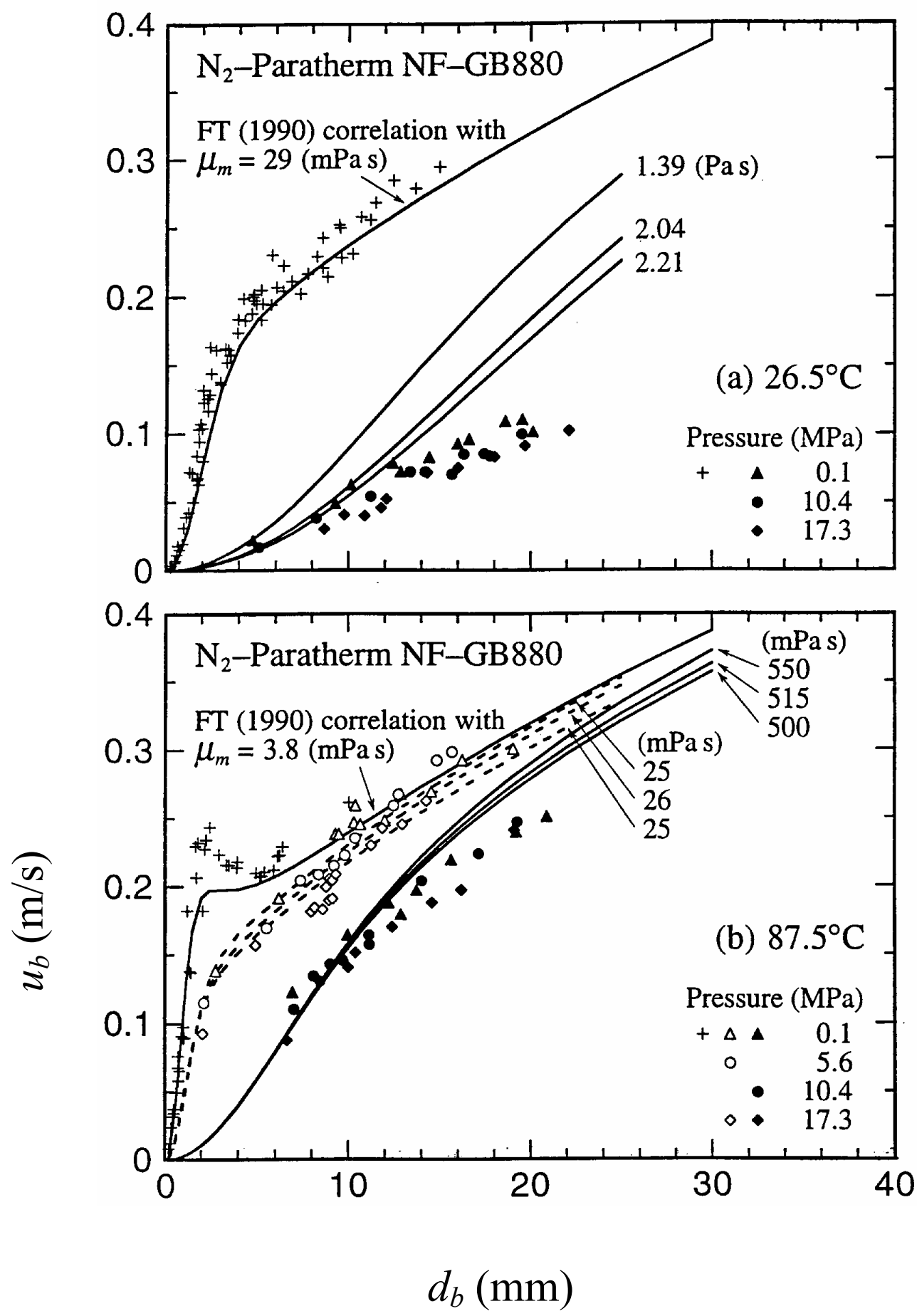

Figure 3 


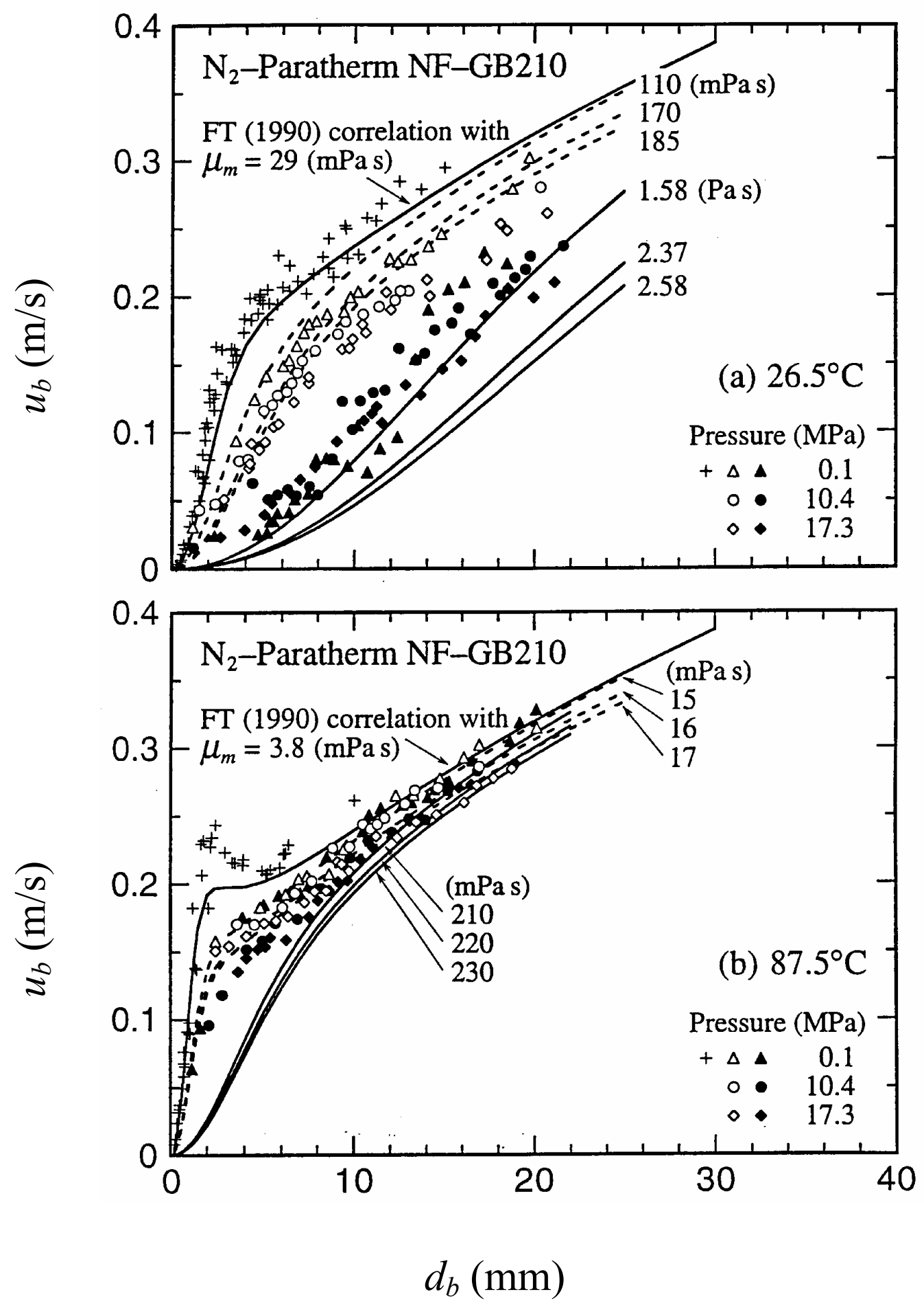

Figure 4 


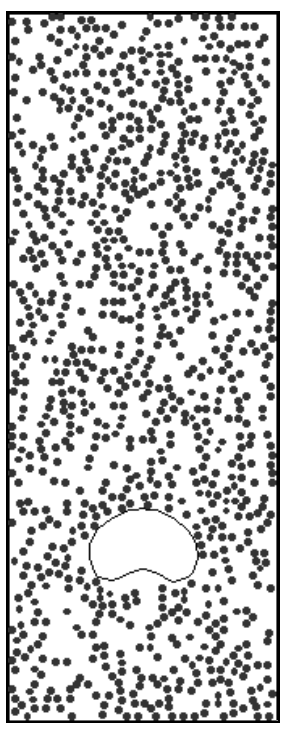

(a) $t=0.06 \mathrm{~s}$

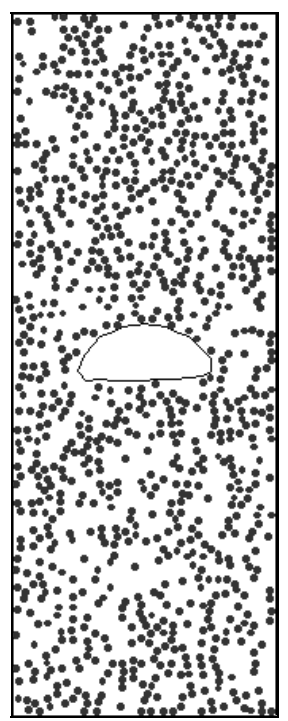

(c) $t=0.26 \mathrm{~s}$

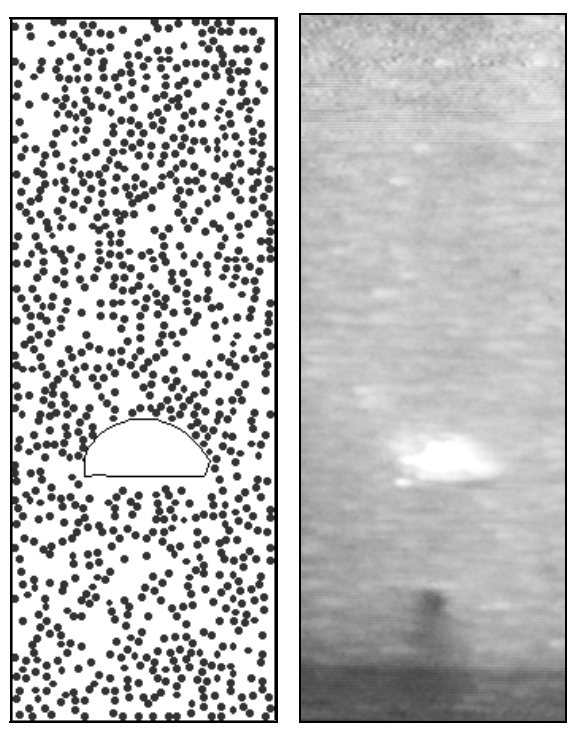

(b) $t=0.16 \mathrm{~s}$

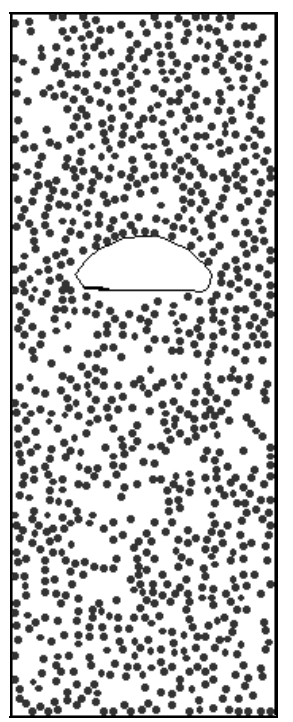

(d) $t=0.36 \mathrm{~s}$

Figure 5 
10.00

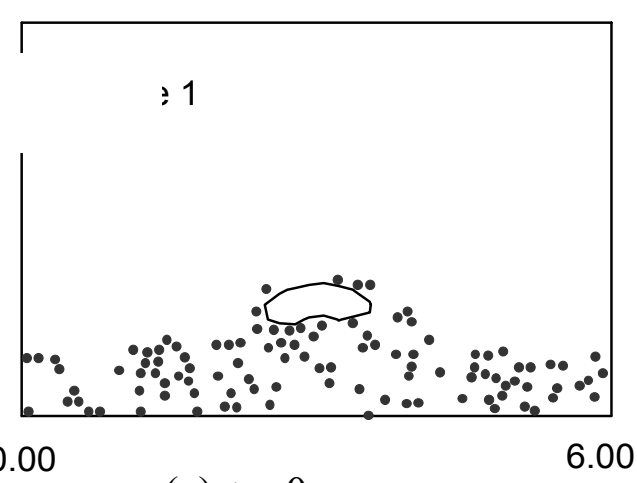

(a) $t=0 \mathrm{~s}$

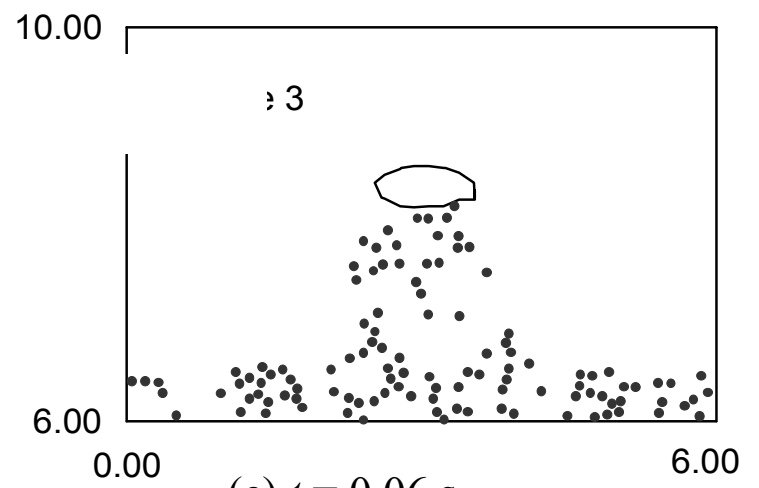

(c) $t=0.06 \mathrm{~s}$
10.00

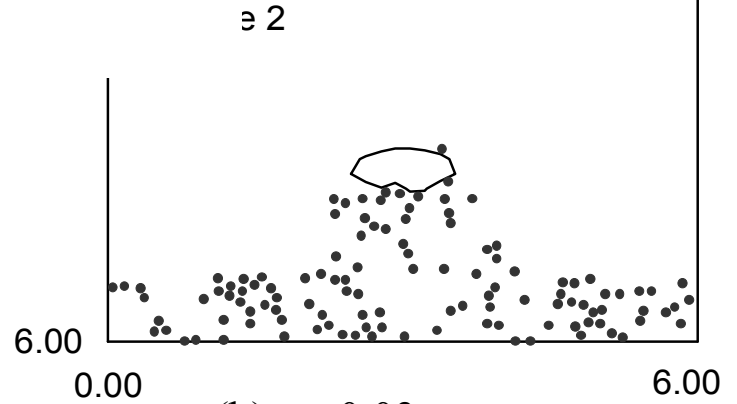

(b) $t=0.03 \mathrm{~s}$

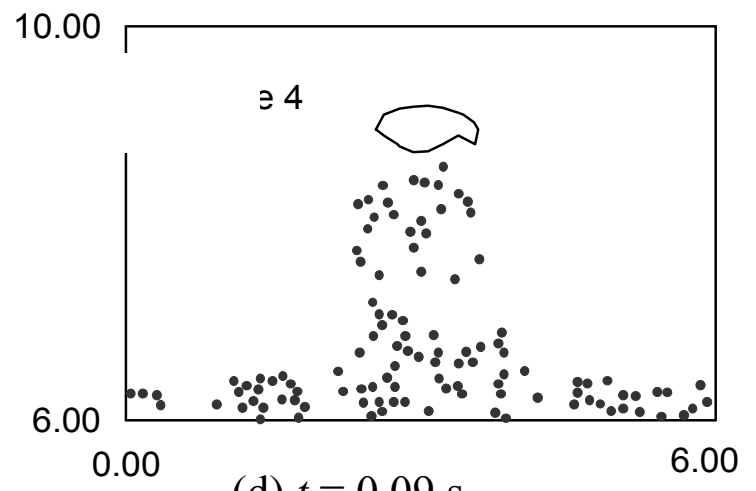

Figure 6 


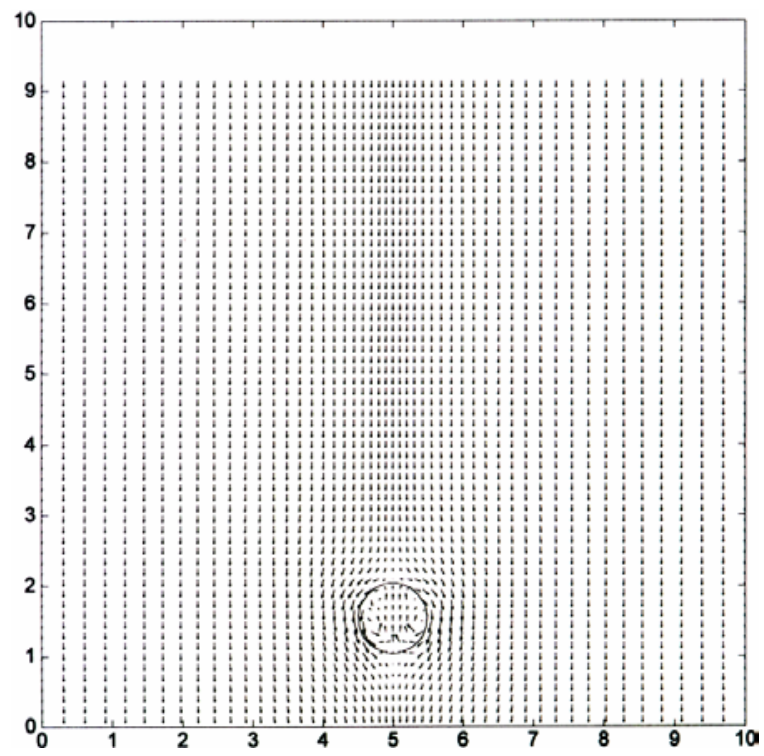

(a) $t=0 \mathrm{~s}$

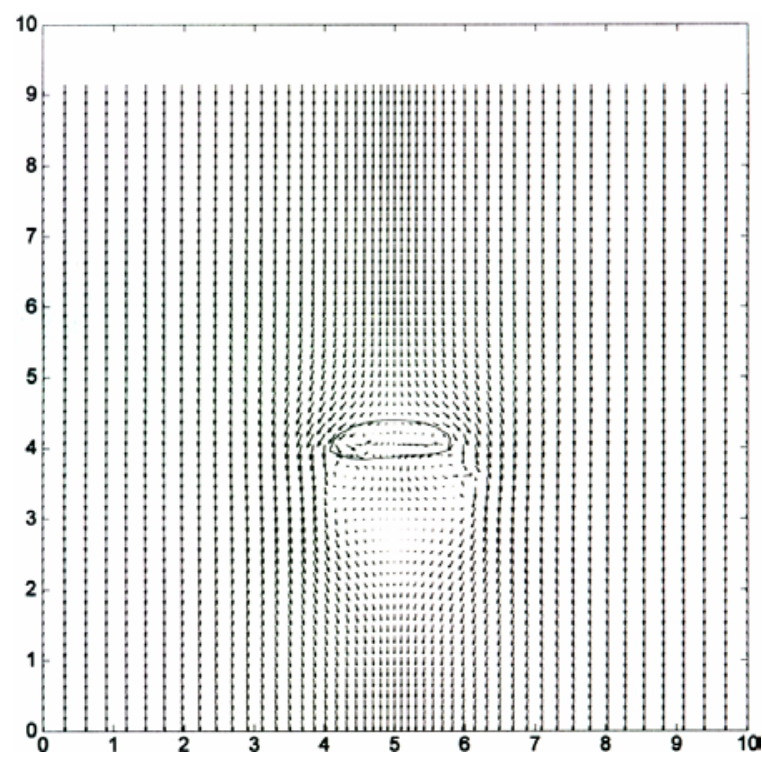

(c) $t=0.12 \mathrm{~s}$

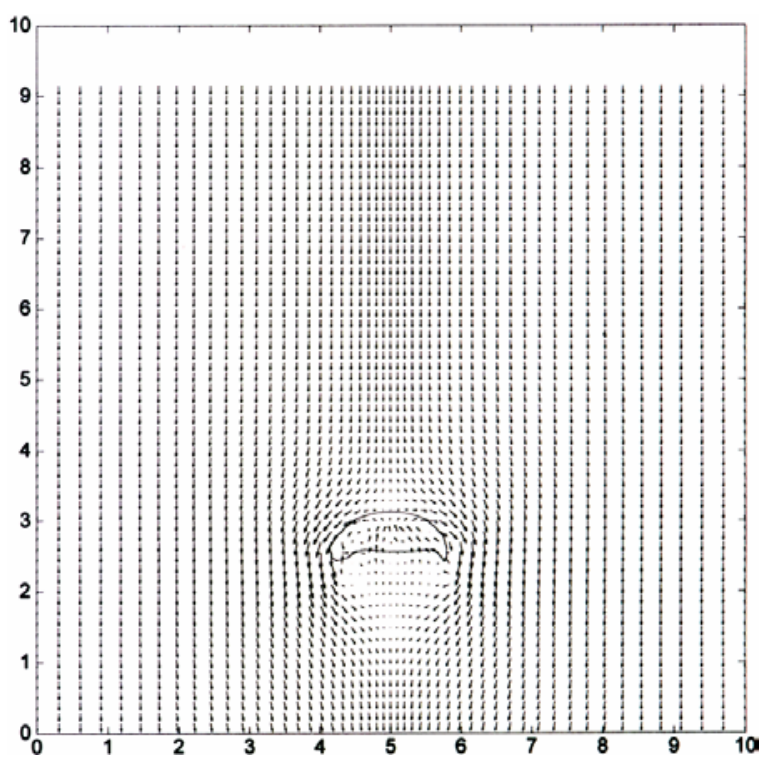

(b) $t=0.06 \mathrm{~s}$

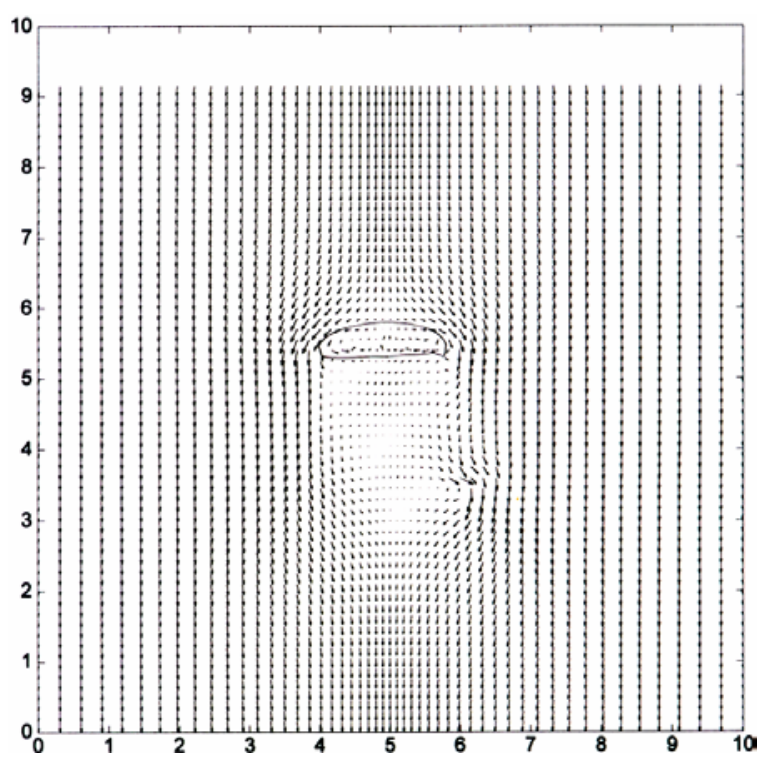

(d) $t=0.18 \mathrm{~s}$

Figure 7 


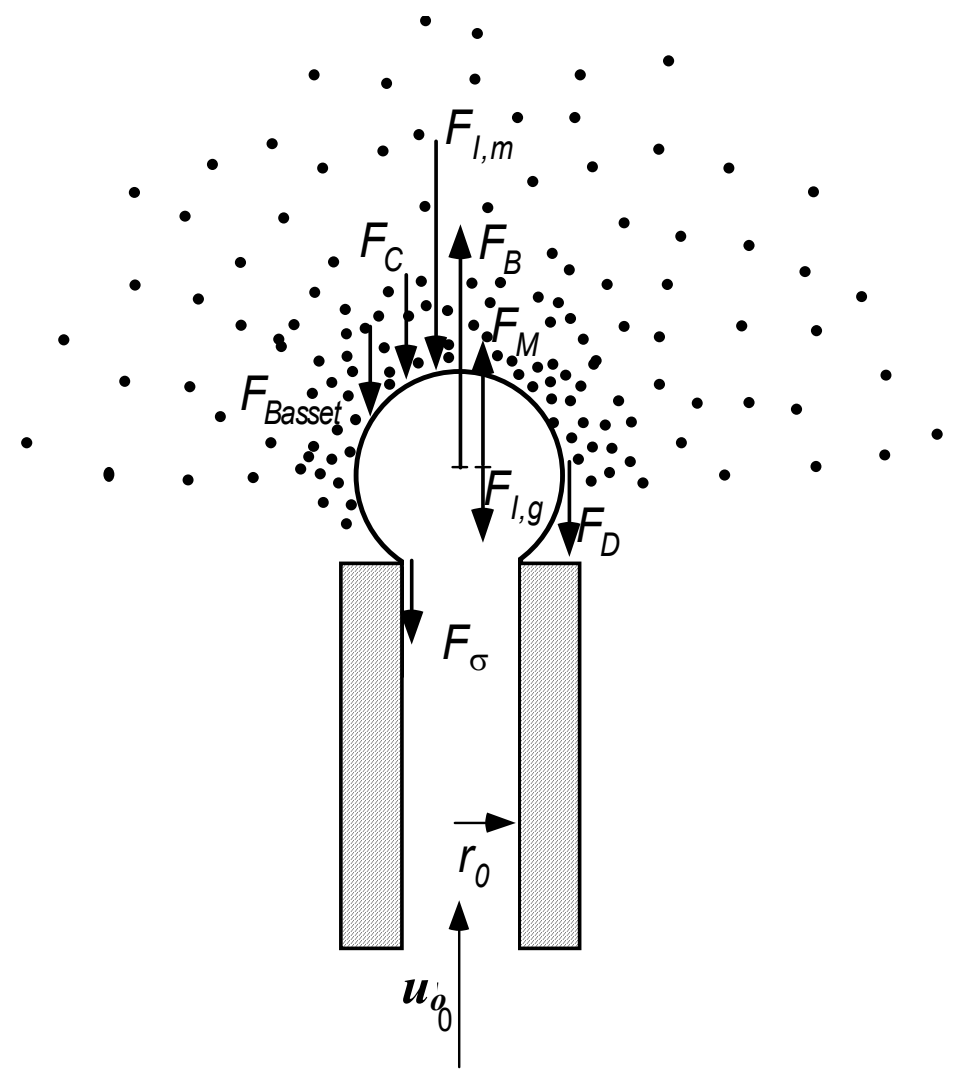

Figure 8 


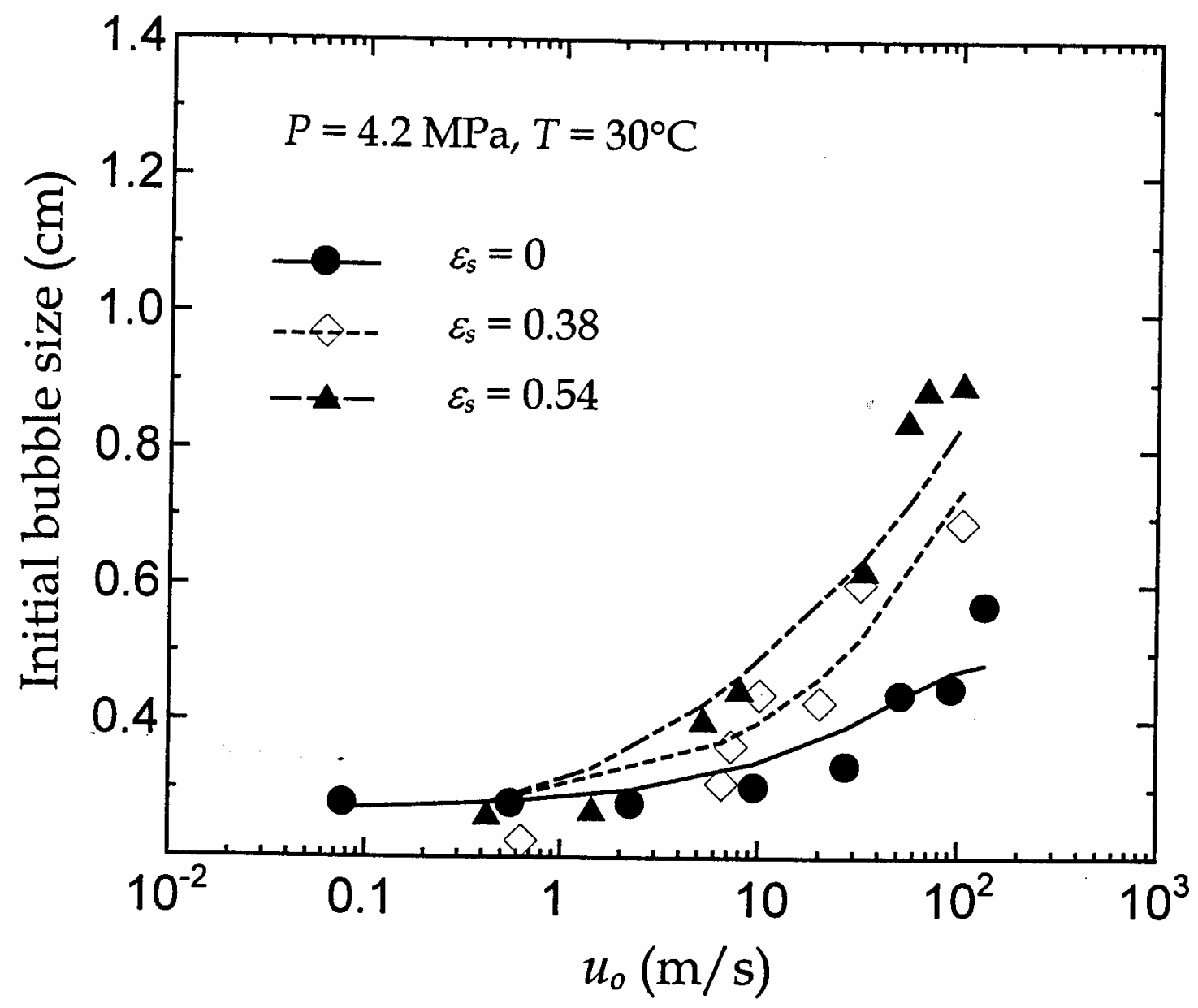

Figure 9 


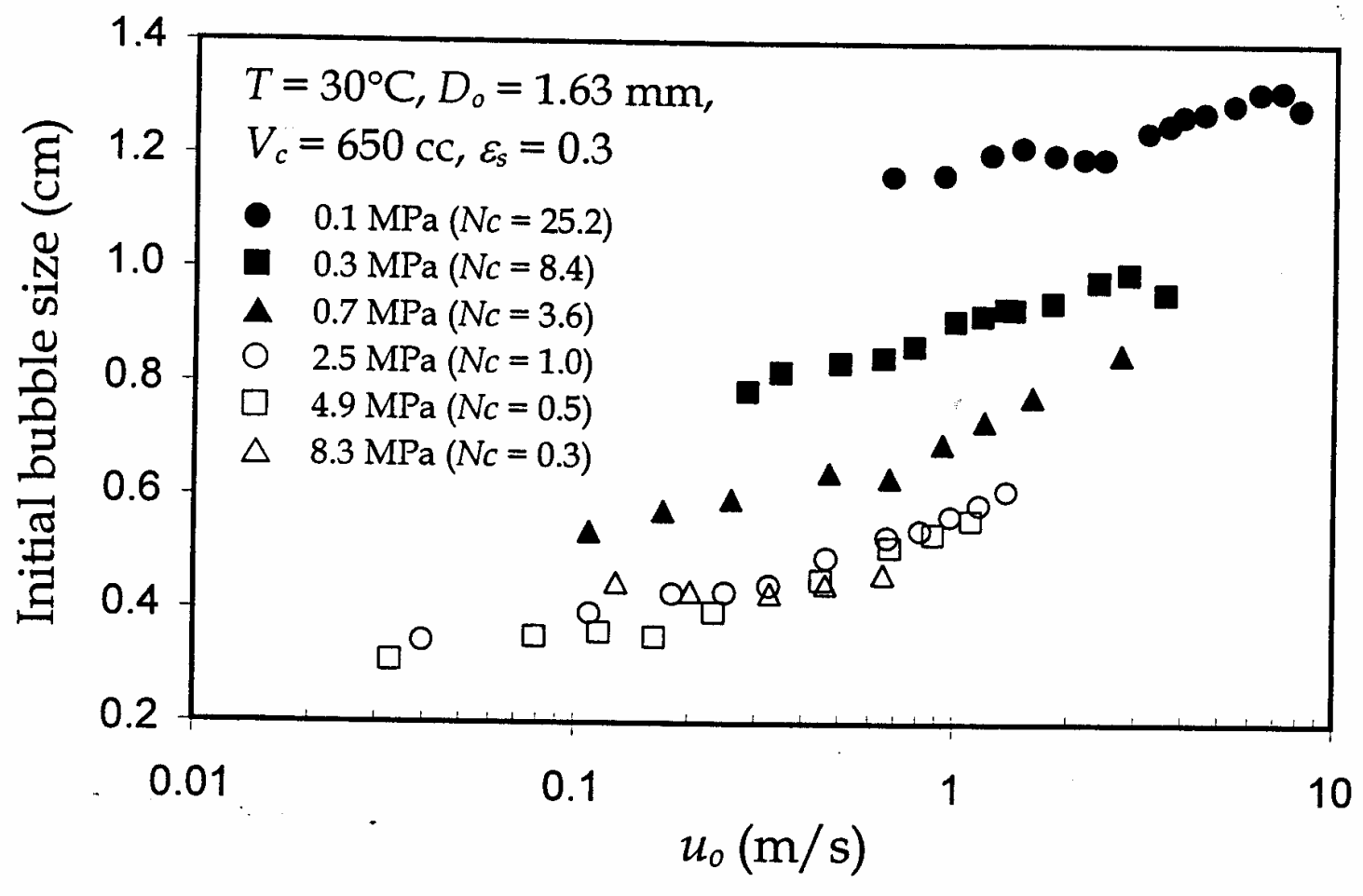

Figure 10 


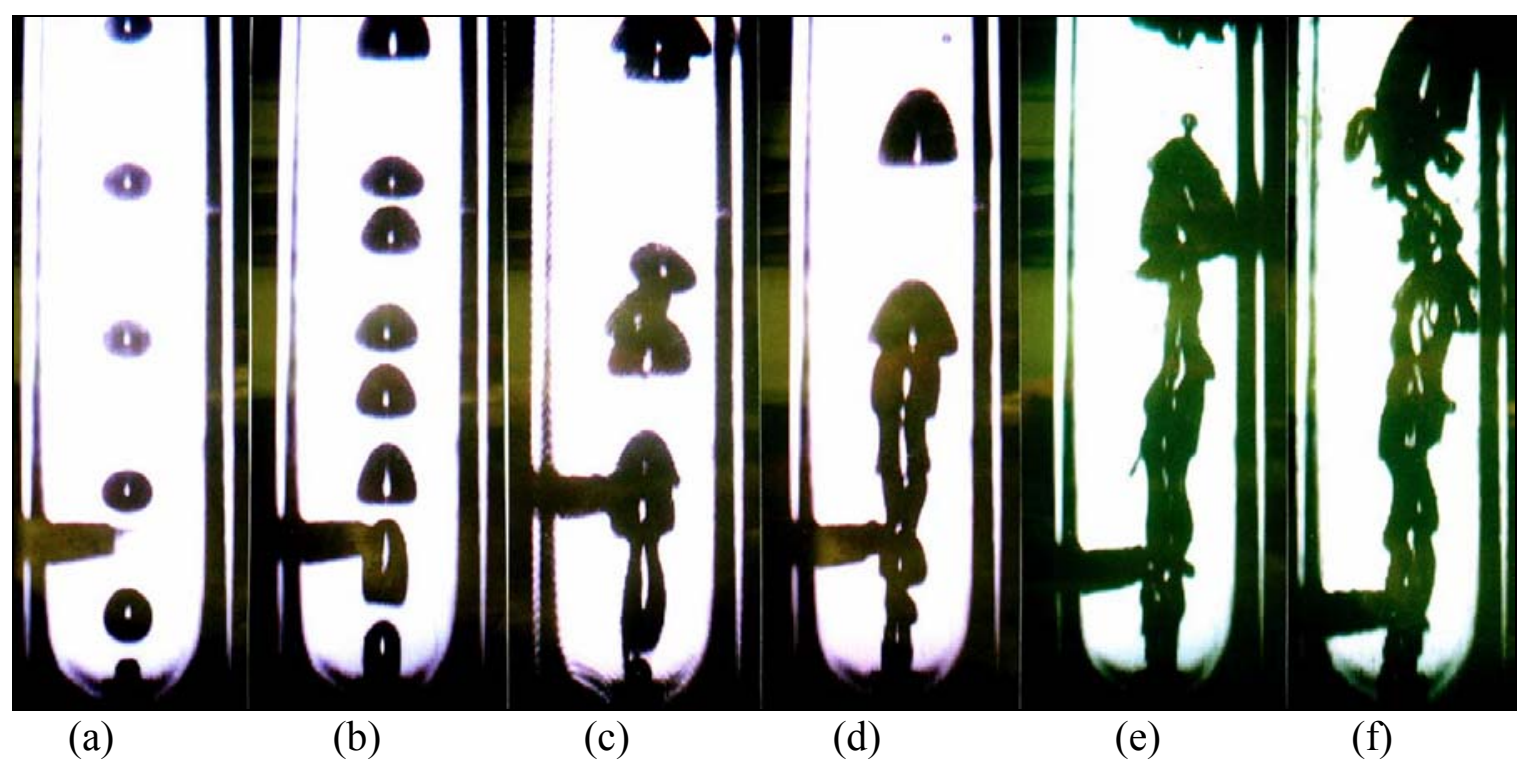

Figure 11 


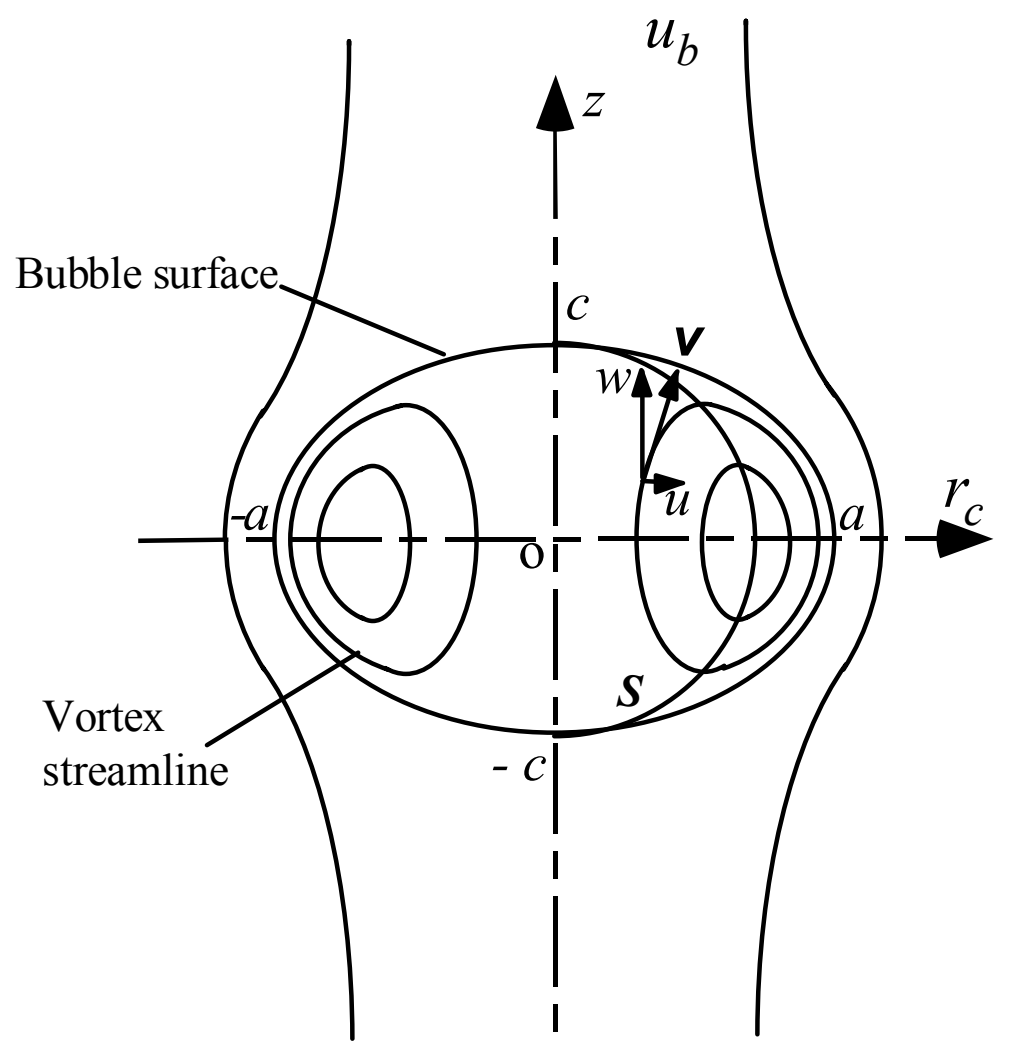

Figure 12 


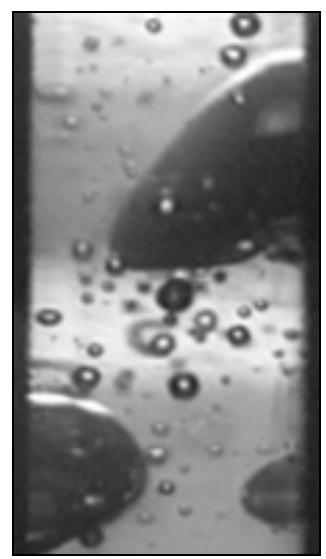

(a) $t=0$

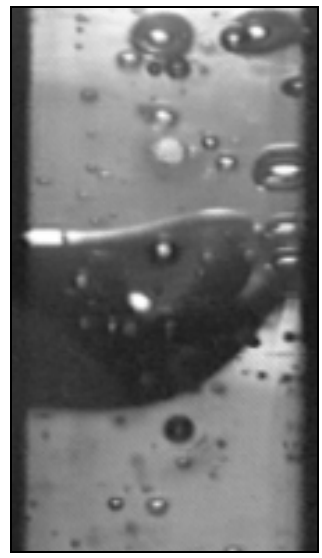

(d) $t=0.052 \mathrm{~s}$

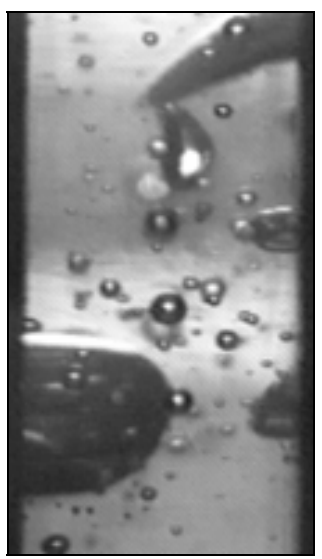

(b) $t=0.017 \mathrm{~s}$

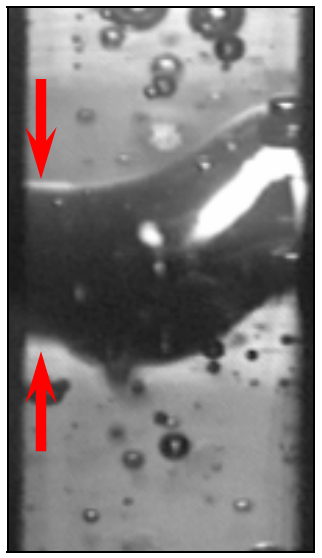

(e) $t=0.068 \mathrm{~s}$

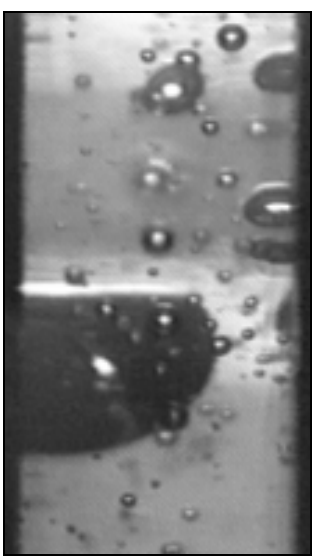

(c) $t=0.034 \mathrm{~s}$

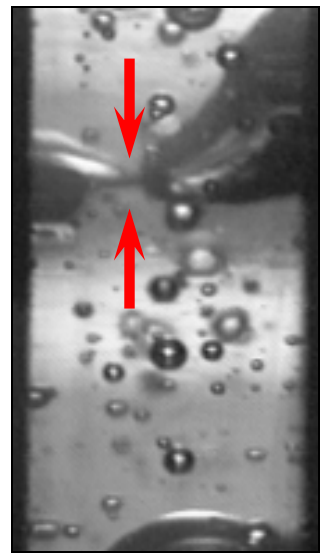

(f) $t=0.085 \mathrm{~s}$

Figure 13 


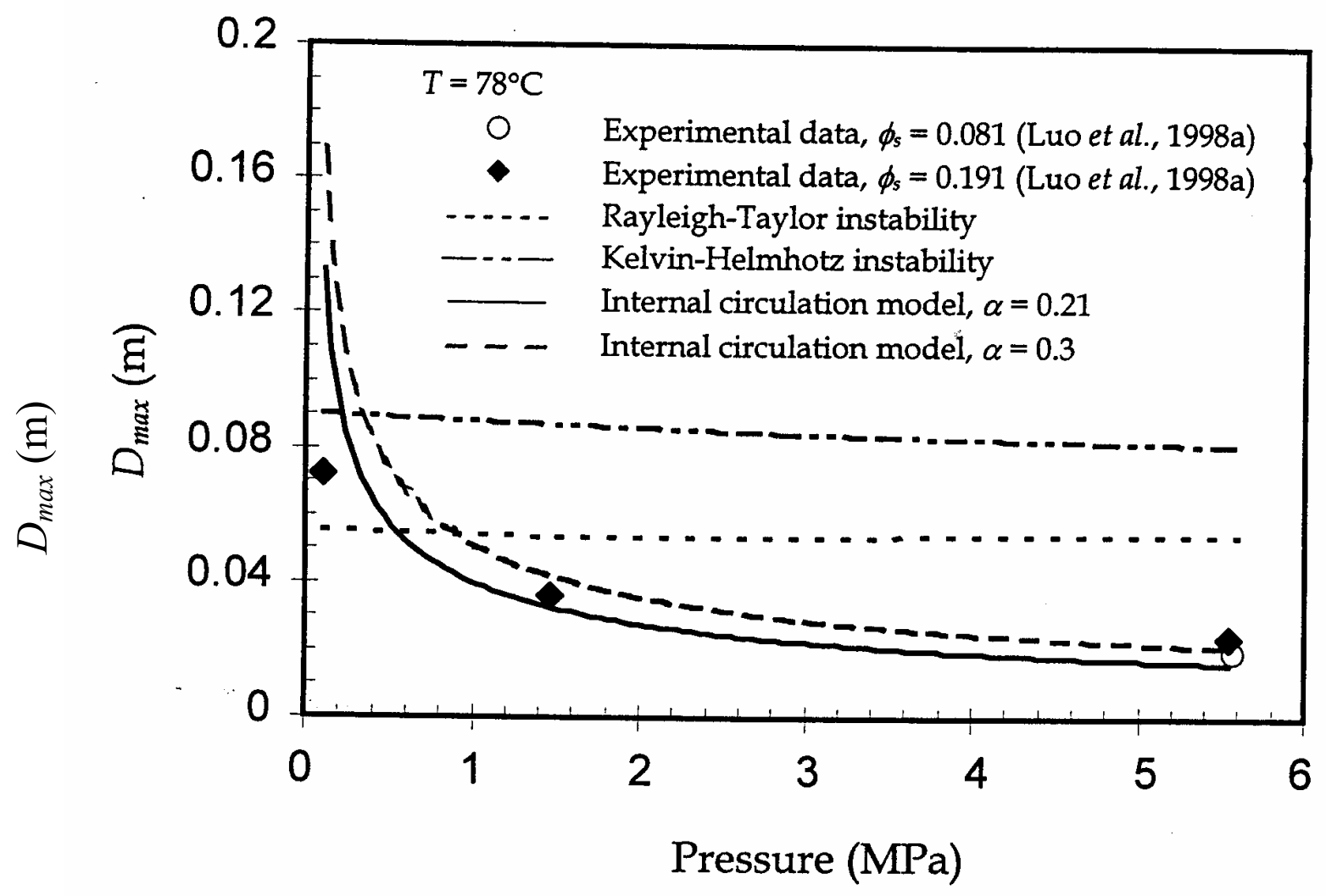

Figure 14(a) 


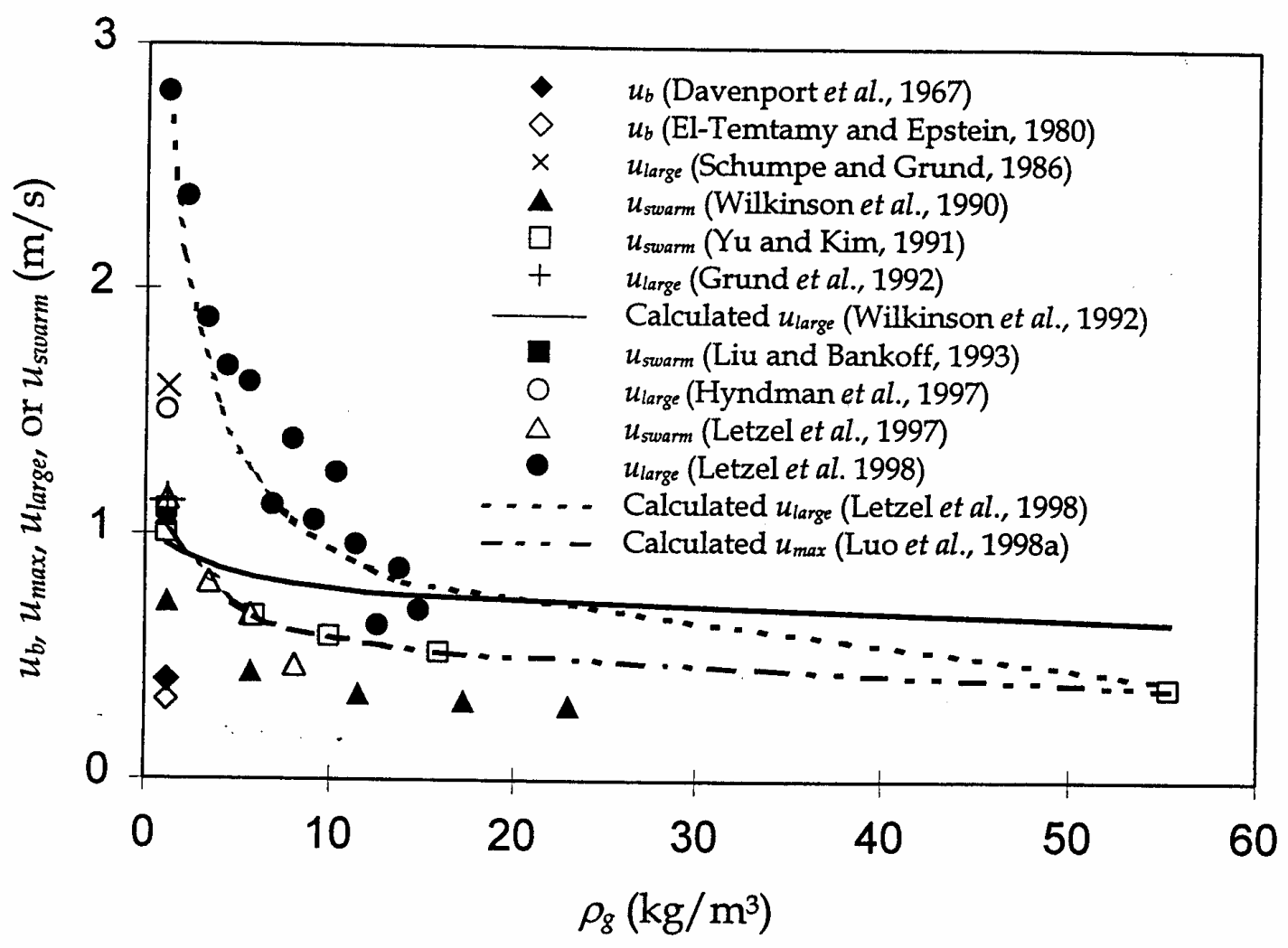

Figure 14(b) 


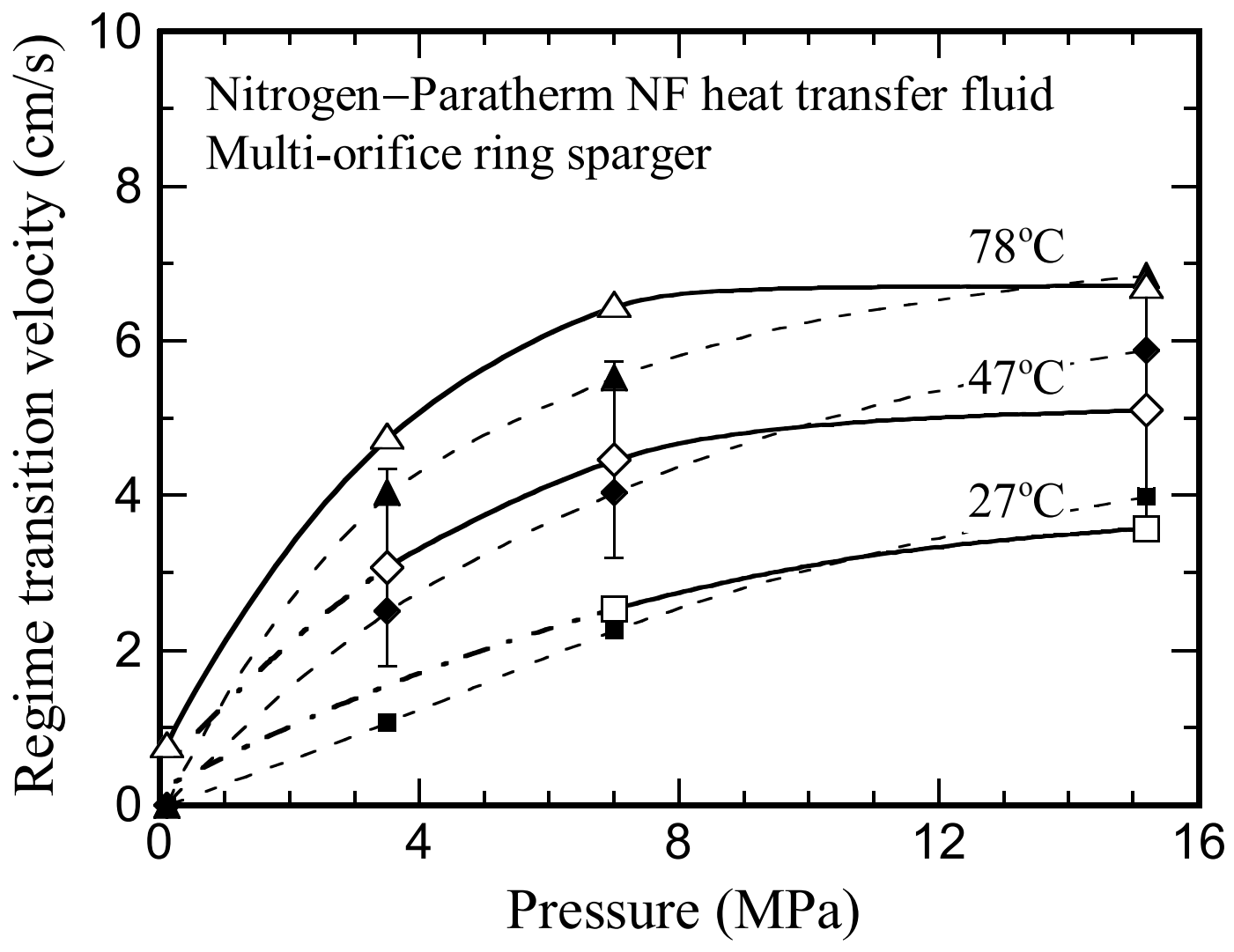

Figure 15(a) 


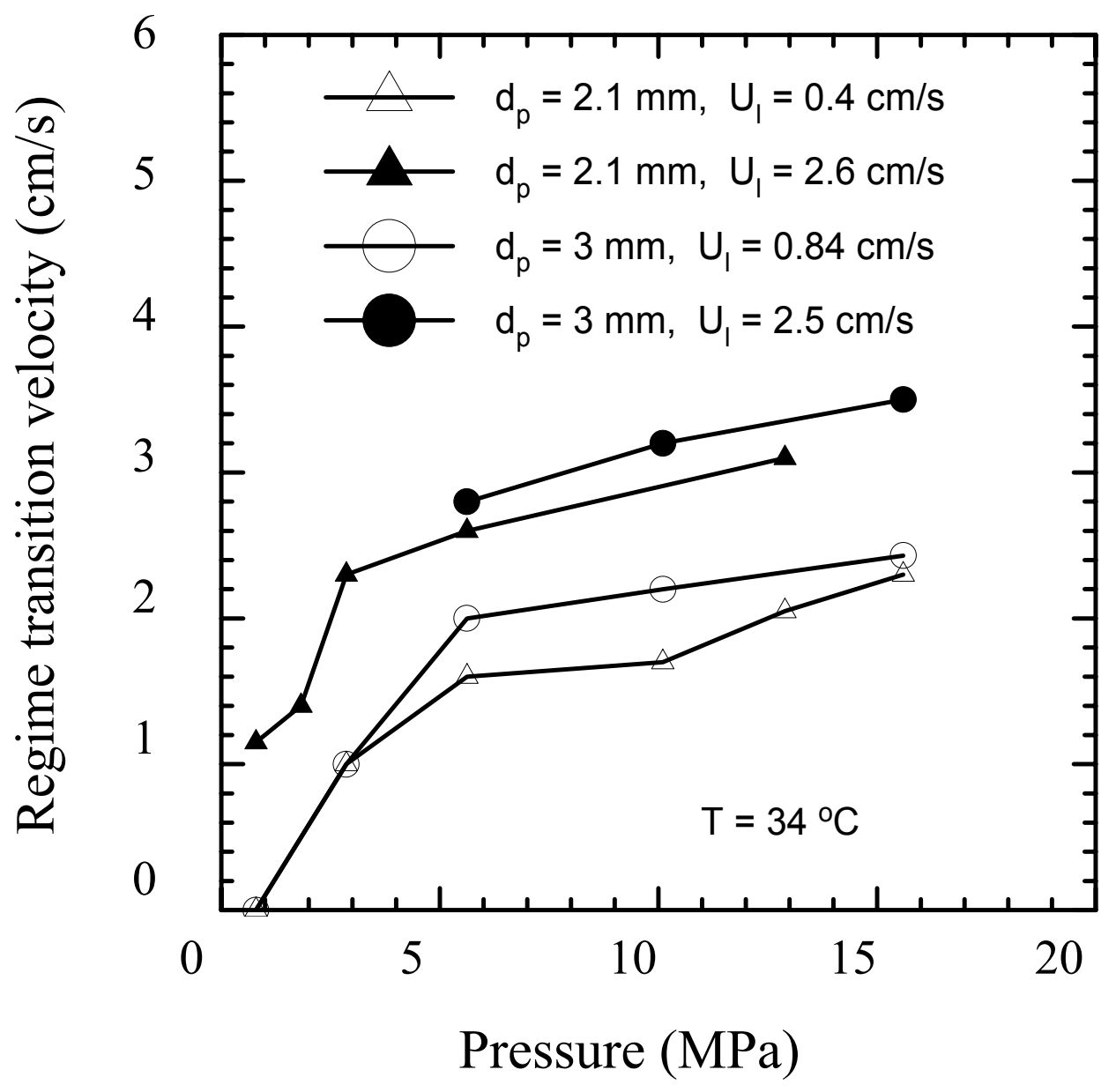

Figure 15(b) 


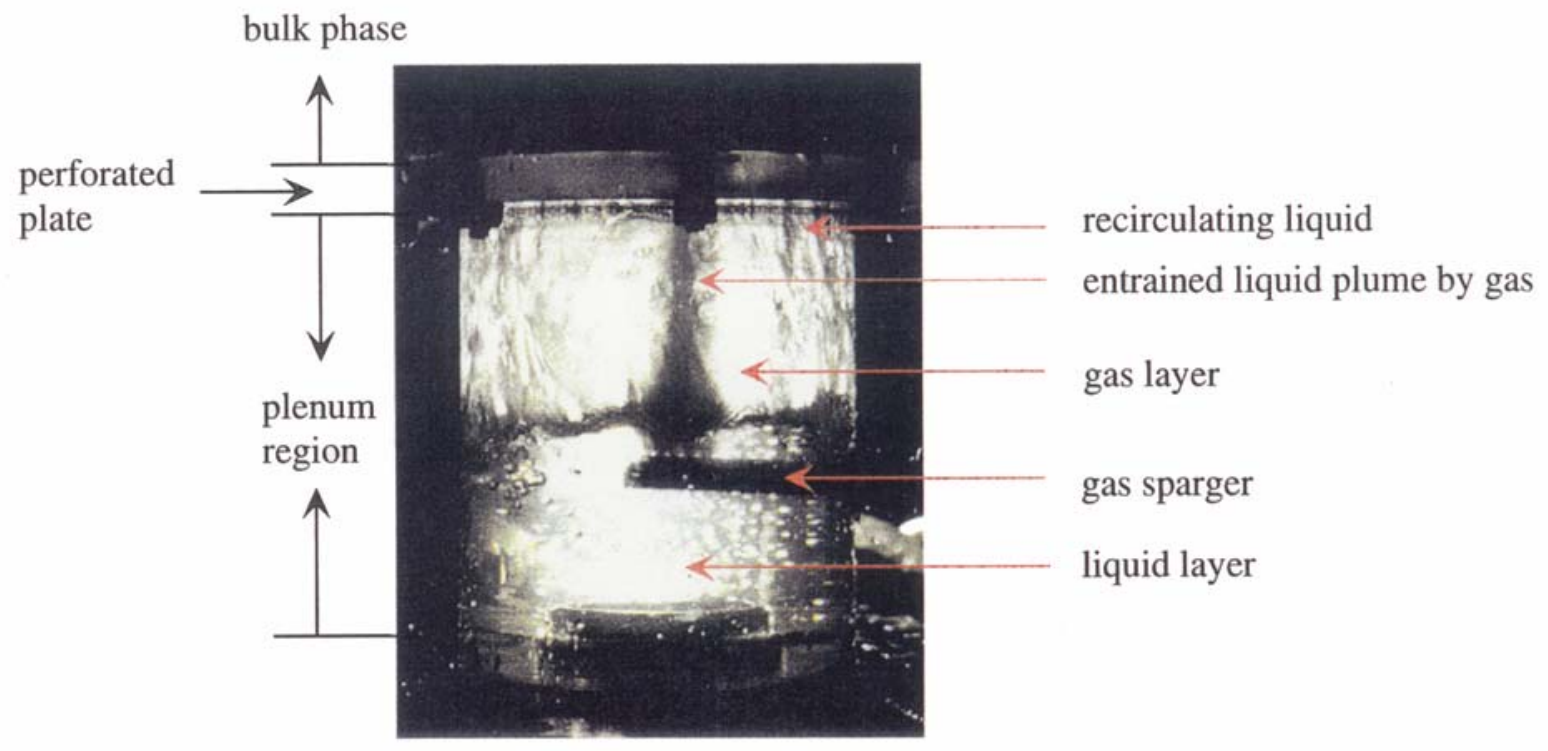

Figure 16 


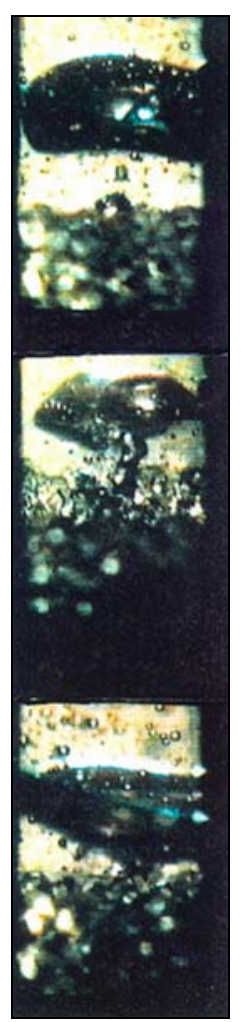

(a)

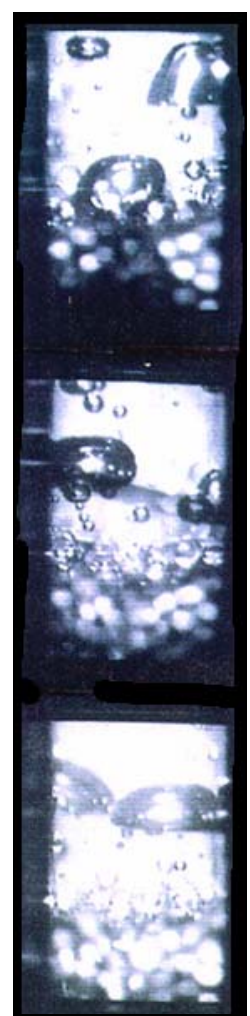

(b)

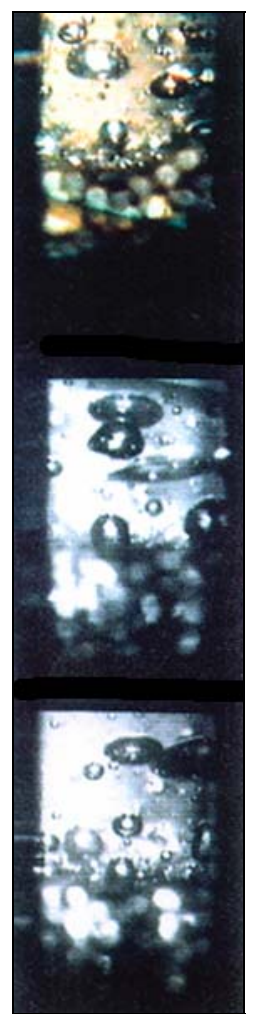

(c)

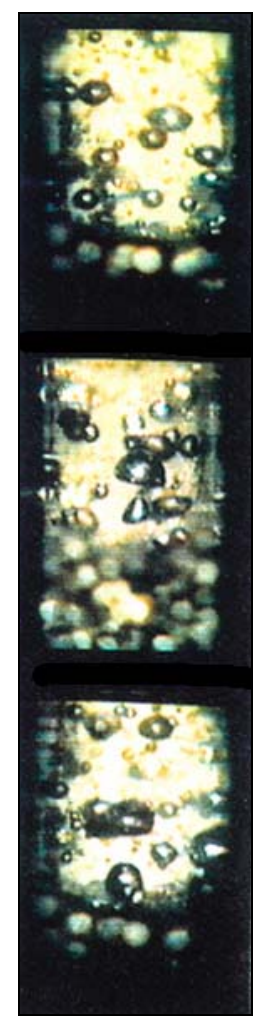

(d)

Figure 17 

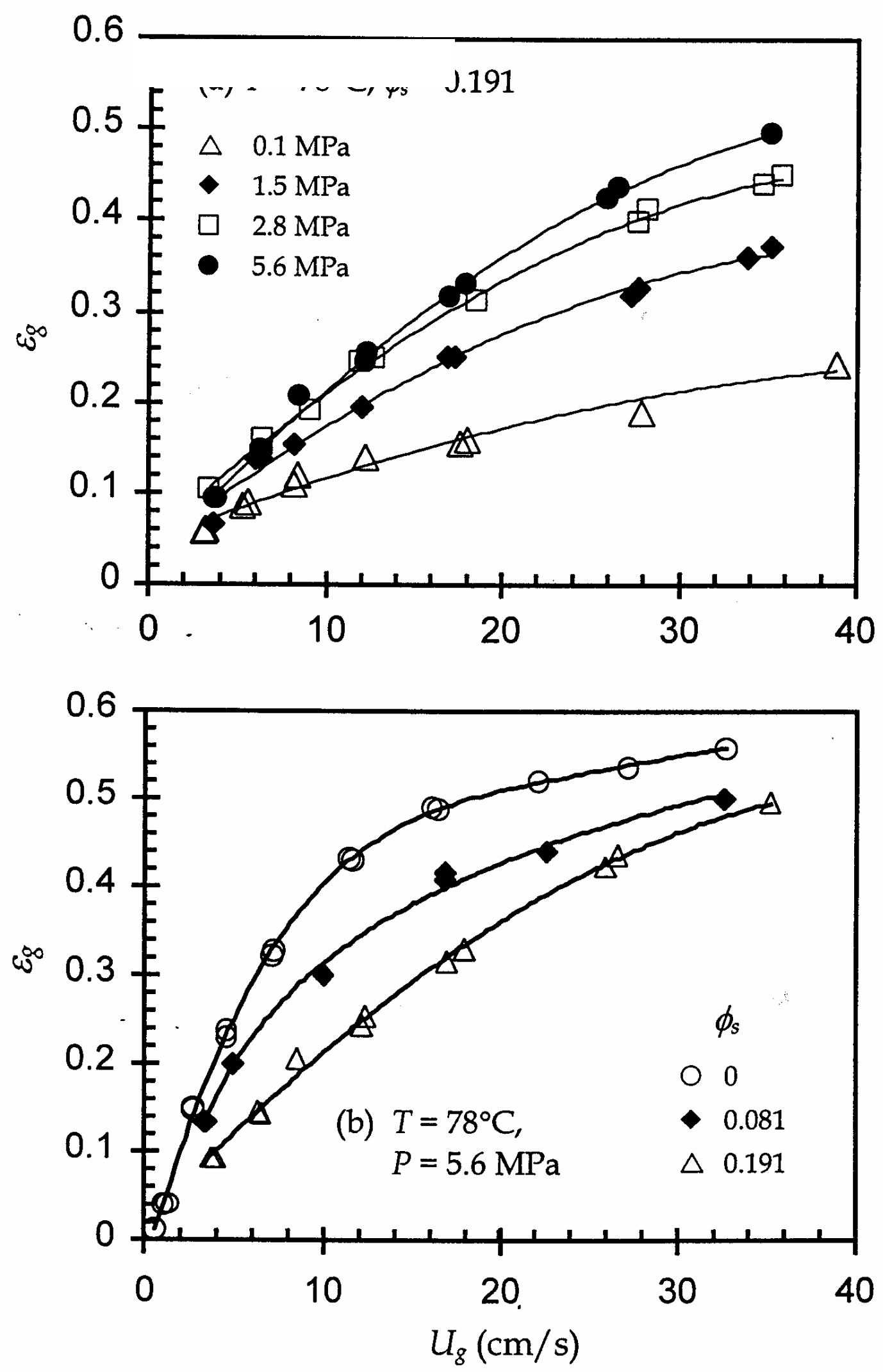

Figure 18 

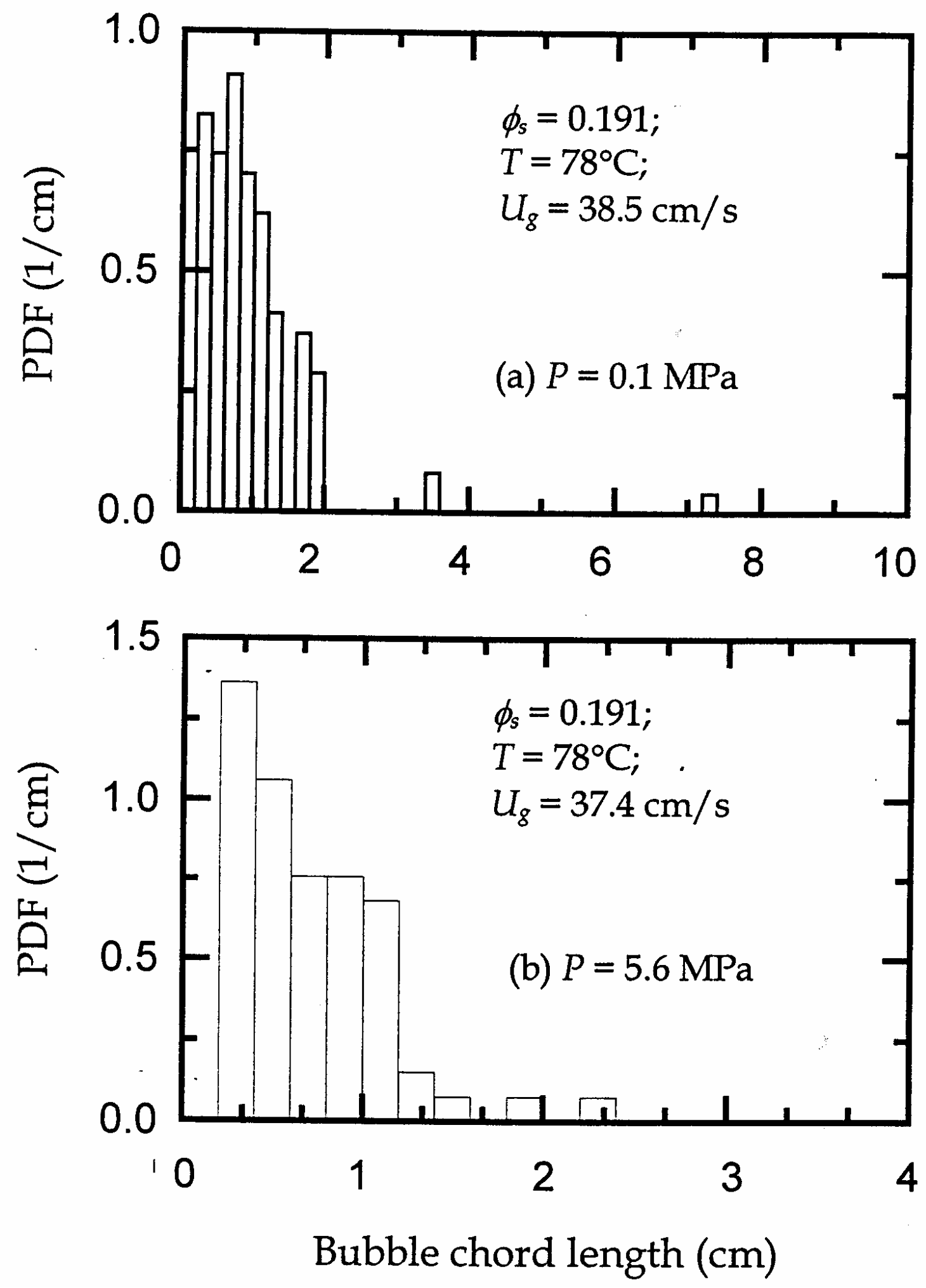

Figure 19 


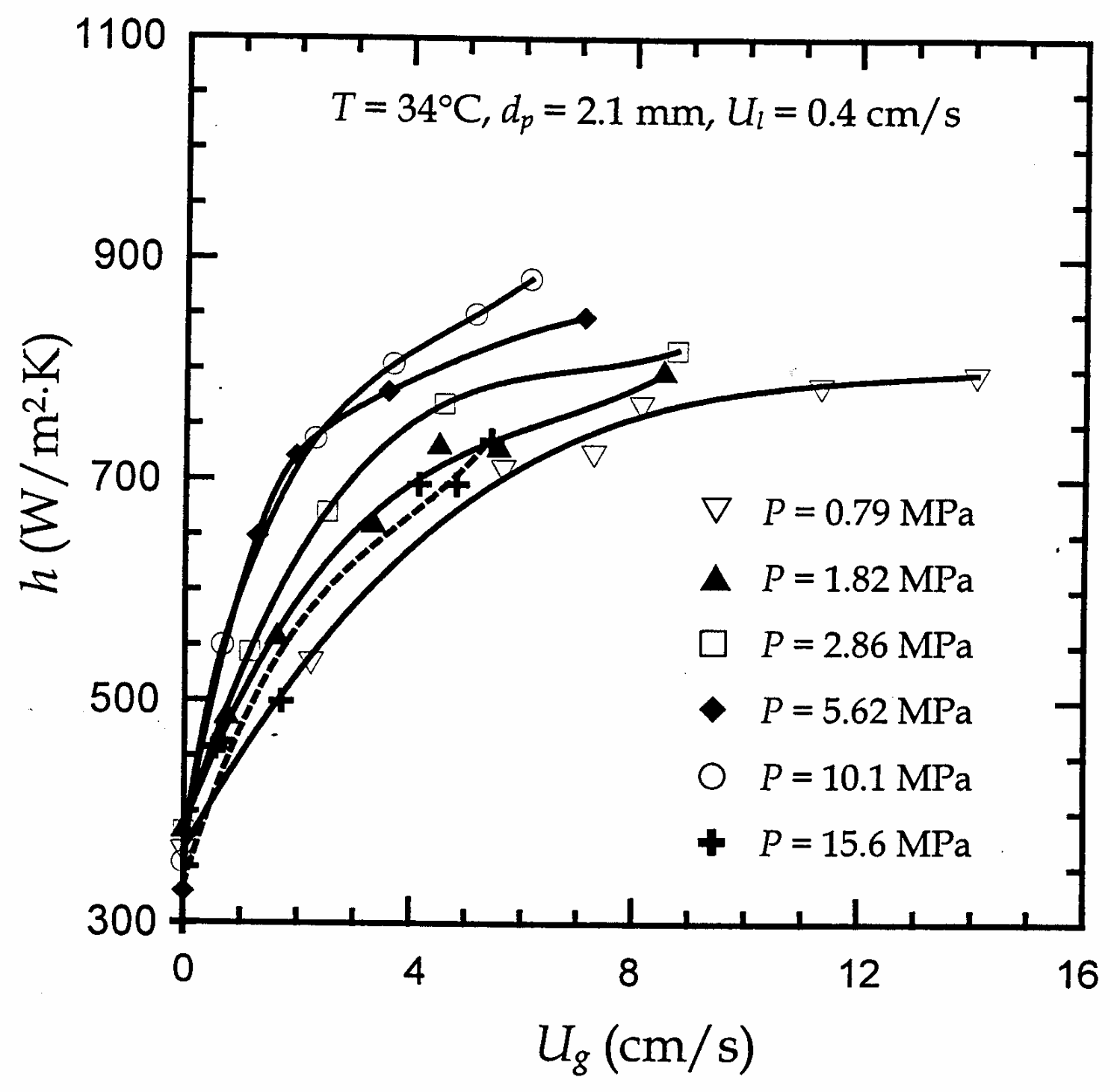

Figure 20 


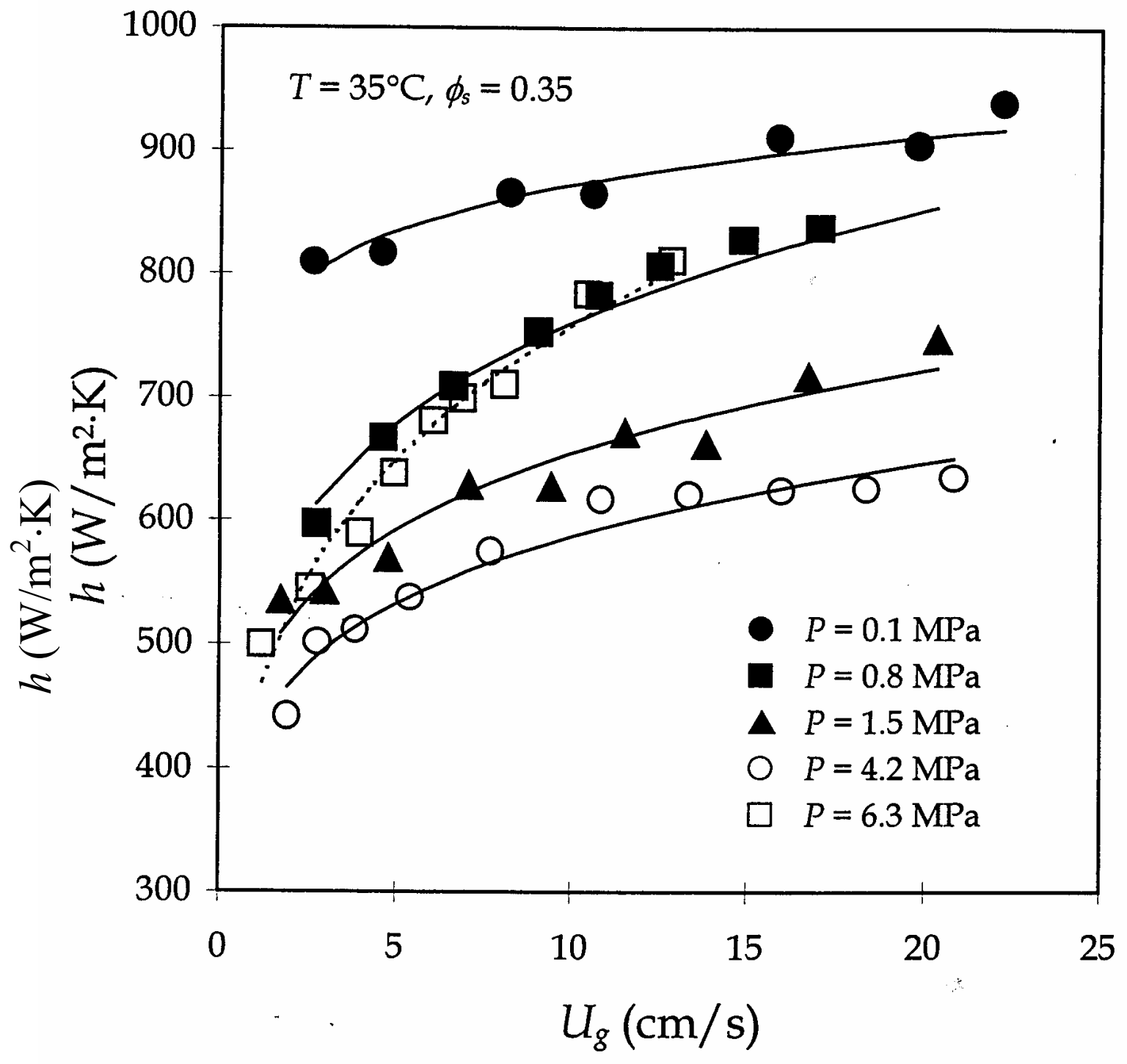

Figure 21 


\section{Appendix B}

a) Predictions of radial gas hold up profiles in Bubble Column reactors

b) Predictions of Axial Liquid Velocity Profile in Bubble Columns 


\title{
Predictions of radial gas hold up profiles in bubble column reactors
}

\author{
Yuanxin $\mathrm{Wu}^{*}$, Boon Cheng Ong and M.H. Al-Dahhan \\ Chemical Reaction Engineering Laboratory, \\ Washington University, St. Louis, MO 63130, USA
}

\begin{abstract}
Gas holdup and its profile are important parameters to be characterized in bubble column reactors. Proper prediction of the radial gas holdup profiles is necessary for determining liquid mixing, flow regime transition, heat and mass transfer. In this study, the following gas holdup profile form, which can be fitted to the observed profiles, is proposed:

$\varepsilon_{G}=\bar{\varepsilon}_{G}\left(\frac{n+2}{n+2-2 c}\right)\left[1-c(r / R)^{n}\right]$

The parameters $\mathrm{n}$ and $\mathrm{c}$ needed to describe the gas holdup profile are correlated with appropriate dimensionless groups.

$n=2.188 \times 10^{3} \operatorname{Re}_{G}{ }^{-0.598} \mathrm{Fr}_{g}{ }^{0.146} \mathrm{Mo}_{L}^{-0.004}$

$c=4.32 \times 10^{-2} \operatorname{Re}_{G}^{0.2492}$

However, the cross-sectional average gas holdup, $\mathcal{E}_{G}$, can be estimated using the available correlations for overall gas holdup. The agreement between the correlation predictions and experimental data is reasonable over wide range of operating conditions. (C) 2001 Elsevier Science Ltd. All rights reserved.
\end{abstract}

Key words: bubble columns, gas holdup profiles, correlation

\section{Introduction}

Gas holdup profile is one of the most important parameters in bubble column reactors. The spatial variation of gas hold up gives rise to pressure variation, which results in liquid recirculation in the bubble column. This liquid recirculation governs the rate of mixing, heat transfer and mass transfer. The ability to predict radial gas holdup profiles in bubble column reactors would help us in determining the flow regimes, liquid mixing, heat and mass transfer better. This should make bubble column scale-up more reliable.

The existence of a pronounced radial hold up profile is the characteristic of the heterogeneous regime of flow in the column which generates strong liquid recirculation. The magnitude of gas holdup radial gradients and the magnitude of liquid velocity driven by the gas depend on superficial gas velocity, column diameter, the nature of the gas-liquid system and the operating conditions (pressure and temperature of the reactor).

During the past three decades, a number of experimental measurements of gas holdup and gas hold up profile have been reported in the literature and have been summarized by Joshi et at.(1998). A

\footnotetext{
${ }^{*}$ Current Address: Wuhan Inst. Of Chem. Tech., Wuhan, Hubei 430073, P.R.China

Email:wyx4@public.wh.hb.cn
} 
variety of techniques, such as optical fiber probes, gamma ray densitometry, particle image velocimetry, and gamma ray and X-ray attenuation together with computer tomography have been employed for the local gas holdup measurements. Due to the complexity of the system, no fundamental equation is available at present for prediction of the gas hold up profiles in bubble columns, There are a number of empirical equations, similar in form, that can be fitted to the observed holdup profiles.

Nassos and Bankoff (1967) proposed the following equation for the radial holdup profile:

$$
\varepsilon_{G}=\widetilde{\varepsilon}_{G}\left(\frac{n+2}{n}\right)\left[1-(r / R)^{n}\right]
$$

In eq(1), $\widetilde{\varepsilon}_{G}$, which is the radial chordal average gas hold up along the column diameter, and the exponent $n$ are parameters and $r / R$ is the dimensionless radial position. The value of parameter $n$ is indicative of the steepness of the holdup profile. When $n$ is large the profile is flat, for small $n$ profile is steep.The steepness of holdup profile is reflected in the intensity of liquid circulation. Ueyama and Miyauchi (1979) reviewed the published literature and modified the above equation as follows to include the possibility of finite gas hold up close to the wall.

$\varepsilon_{G}=\widetilde{\varepsilon}_{G}\left(\frac{n+2}{n}\right)\left[1-c(r / R)^{n}\right]$

In eq.(2), $\mathrm{c}$ is an additional parameter which is indicative of the value of gas holdup near the wall. If $\mathrm{c}=1$ there is zero hold up close to wall, if $\mathrm{c}=0$ hold up is constant with changing $\mathrm{r} / \mathrm{R}$.

Luo and Svendsen (1991) used eq.(2) represented in terms of mean cross-sectional profile as given below:

$$
\varepsilon_{G}=\bar{\varepsilon}_{G}\left(\frac{n+2}{n+2-2 c}\right)\left[1-c(r / R)^{n}\right]
$$

By applying equation (3) to data, $\mathrm{n}$ was found from 1.4 to11, and c from 0.5 to 1 according to the findings of different authors (Joshi e tal.,1998) and based on different systems investigated. In the absence of a firm theoretical prediction of the radial gas hold up profile correlations are needed for evaluating $\mathrm{n}$ and $\mathrm{c}$ based on the knowledge of the general operating variables and physical properties of the system in order to estimate the gas holdup profile by equation (3). In this work, such correlations have been developed as discussed below.

\section{Correlation Development}

Extensive gas holdup and gas holdup profile data have been acquired in the Chemical Reaction Engineering Laboratory (CREL) over the years under DOE contract DE2295PC95051 on the bubble column hydrodynamics, by employing gamma ray Computed Tomography $(\mathrm{CT})$ over a wide range of superficial gas velocities (from $2 \mathrm{~cm} / \mathrm{s}$ to $60 \mathrm{~cm} / \mathrm{s}$ ), at different pressures $(0.1 \mathrm{MPa}$ to $1.0 \mathrm{MPa}$ ) with 5 different gas distributors and in columns ranging in diameter from $0.19-0.44 \mathrm{~m}$. The majority of the gas holdup profiles were measured in air-water system. However, air-drakeoil (light mineral oil) and air-propanol systems were also used at different operating conditions. The reproducibility of the measured gas holdup profiles was within $\pm 3 \%$. By analyzing the experimental results carefully it was found that the shape of hold up profiles changes most significantly with superficial gas 
velocities. However, pressure affects the shapes of hold up profiles but it has less effect compared to superficial gas velocity within the range of pressure and superficial gas velocity. Gas distributor does affect hold up in a certain range of gas velocities but it has a minor effect on gas holdup profiles particularly in the fully developed region and at high gas velocity. The shape of the gas holdup profile at different column heights seems to be unchanged at given gas velocity once the measurement has been taken at a certain distance from the distributor (2L/D or higher). Column diameter has been reported to have an effect on gas holdup profile (Kumar et al., 1997).

Based on the experimental observations and dimensional analysis, the following functional definition was proposed for parameters $\mathrm{n}$ and $\mathrm{c}$ :

$n=a f\left(\operatorname{Re}_{G}, M o_{L}, F r_{G}\right) ; c=\beta \zeta\left(\operatorname{Re}_{G}\right)$

The above dimensionless groups, $\mathrm{Re}_{\mathrm{G}}, \mathrm{Mo}_{\mathrm{L}}, \mathrm{Fr}_{\mathrm{G}}$, which are defined below reflect the effect of velocity and pressure, which change the density of the gas and has an effect on gas holdup profile, and the effect of gas and liquid physical properties. By fitting roughly two third of the available experimental data from the database consisting of our experiments and those in the literature (the remaining about one third of the experimental data are used to evaluate the developed correlations), the correlations listed below are generated for $\mathrm{n}$ and $\mathrm{c}$ :

$$
\begin{aligned}
& n=2.188 \times 10^{3} \operatorname{Re}_{G}{ }^{-0.598} \operatorname{Fr}_{g}{ }^{0.146} M o_{L}^{-0.004} \\
& c=4.32 \times 10^{-2} \operatorname{Re}_{G}^{0.2492}
\end{aligned}
$$

where

$$
\operatorname{Re}_{G}=\frac{D U_{S g}\left(\rho_{L}-\rho_{G}\right)}{\mu_{L}}, F r_{G}=\frac{U_{S g}^{2}}{g D}, M o_{L}=\frac{g \mu_{L}^{4}}{\left(\rho_{L}-\rho_{G}\right) \sigma_{L}^{3}}
$$

Equation (4) and (5) along with equation (3) are utilized for prediction of the gas holdup profiles for the whole set of the experiment data available. The cross sectional mean gas holdup values used in this study were evaluated from the experimental data. However, a favorite correlation for the overall gas holdup can be used to estimate $\bar{\varepsilon}_{G}$ (Kemoun, Ong, Gupta, Al-Dahhan \& Dudukovic, 2000).

As mentioned above, the majority of experimental data used were obtained by using an air-water system. Hence, based on the fitting performed in this study, $\mathrm{n}$ is almost independent of $\mathrm{Mo}_{\mathrm{L}}(\mathrm{n} \alpha$ Mo ). However, it was found that the liquid physical properties affect the overall gas holdup (Luo, Lee, Lau, Yang \& Fan, 1999) and the holdup profile (Chen et al., 1998; Joshi et al., 1998). Therefore, at this stage, $\mathrm{Mo}_{\mathrm{L}}$ is included in the correlation to be examined for any future necessary modification as gas holdup profiles become available for a wide enough range of liquid physical properties.

Due to the fact that most of the holdup profiles used were for air-water system, c was found to be only a function of $\mathrm{Re}_{\mathrm{G}}$. Liquid physical properties would affect the parameter $\mathrm{c}$ which needs to be examined further. 


\section{Comparison between the predicted and experimentally measured gas holdup profiles}

\section{Effect of superficial gas velocity}

As mentioned earlier, gas holdup profiles vary significantly with gas velocity. The results are shown in Figure 1.

One can see from Figure 1 that gas holdup profiles become steeper with increased gas velocity (n changes from 3.73 at $8 \mathrm{~cm} / \mathrm{s}$ to 2.02 at $60 \mathrm{~cm} / \mathrm{s}$ ). The steeper holdup profile is, the faster liquid recirculation rate is, hence liquid mixing, heat transfer and mass transfer rate will be improved accordingly. From Figure 1, one can observe that model predicts the experimental results reasonable well (mean relative error is within 15\%).

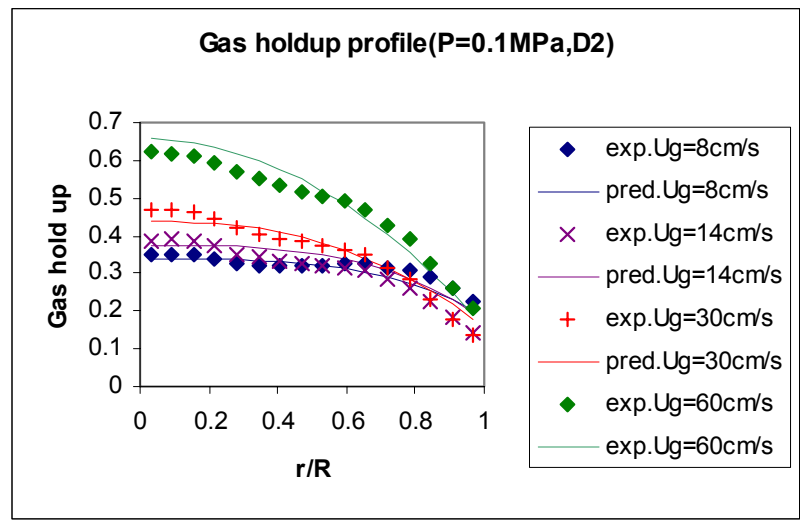

Figure 1: Effect of velocity on gas hold up profile(Diameter of column: $0.15 \mathrm{~m}$, Distributor D2: Perforated plate, Hole diameter: $0.5 \mathrm{~mm}$,Number of holes: 163 , Open area: $0.15 \%$; $\mathrm{P}=0.1 \mathrm{MPa}$;Waterair system)

\section{Effect of reactor pressure}

It is shown that pressure not only changes the gas hold up but also changes the gas holdup profile as well (Joshi etal., 1998).

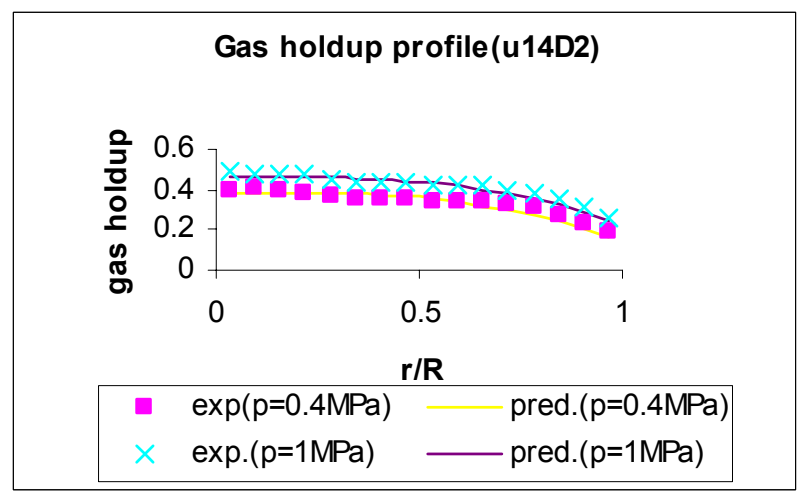

Figure 2: Effect of pressure on gas hold up profile in $0.15 \mathrm{~m}$ reactor diameter $(\mathrm{Ug}=14 \mathrm{~cm} / \mathrm{s}$, distributor D2: Perforated plate, Hole diameter: $0.5 \mathrm{~mm}$, Number of holes: 163, Open area: $0.15 \%$, air-water system) 
At higher pressure smaller bubbles are formed by breakage due to high gas density. Small bubble size increases the overall gas holdup, and as pressure increases, gas bubble size distribution becomes narrow which results in a slightly flatter hold up profile due to the uniform distribution of small bubbles. As shown in Figure 2, at $\mathrm{Ug}=14 \mathrm{~cm} / \mathrm{s}$ and pressure varies from $0.4 \mathrm{MPa}$ to $1 \mathrm{MPa}$ there is no major difference in the shape of gas hold up profile (n values varies from 3.146 to 3.168). Unfortunately, currently there is no gas hold up profile available at higher pressure and higher gas velocity. For the current operation condition, the model predicts the experimental results well (mean relative error is within $14 \%$ ).

\section{Effect of physical properties}

As mentioned earlier, both holdup distribution and liquid recirculation depend on liquid physical properties. A noncoalescing system and coalescing systems have different overall gas holdup and gas holdup profiles as well. Figure 3 illustrates that the proposed correlations predict the experimental results reasonably well for liquids of different physical properties.

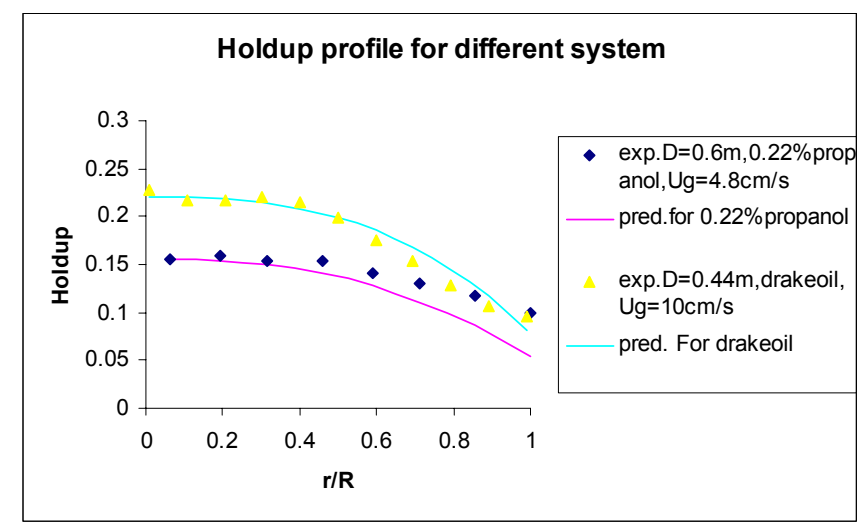

Figure 3: Effect of liquid physical properties on hold up profile $(\mathrm{Ug}=4.8 \mathrm{~cm} / \mathrm{s}, \mathrm{D}=0.6 \mathrm{~m}$, air- $0.22 \%$ propanol in water: Menzel et al., 1990; $\mathrm{Ug}=10 \mathrm{~cm} / \mathrm{s}, \mathrm{D}=0.44 \mathrm{~m}$,air-drakeoil: Chen et al., 1998)

It is noteworthy that two sets of the data presented in Figure 3 are taken from the literature (Menzel et al., 1990; Chen et al., 1998), and are predicted well by the developed correlations ( mean relative error within 17\%). However, larger errors in the correlations predictions are obtained in the region near the wall for the data obtained from Menzel et al. (1990). This would be due to, as mentioned above, the majority of the measured holdup profiles used for the developed correlations (Eqs.(4) and (5)) were obtained for air-water system which affect the dependency of the parameters $n$ and $\mathrm{c}$ on the liquid physical properties.

\section{Effect of column diameter}

As reported in the literature (Joshi et al., 1998) with increase in column diameter D, the liquid recirculation velocity increases as $\mathrm{V}_{\mathrm{c}} \alpha D^{0.3 t 00.4}$. Hence, one would expect a steeper holdup profile in larger column diameter. This is not obvious from Figure 4 due to different superficial gas velocities used in columns of different diameter and this is the only available data at the moment. 


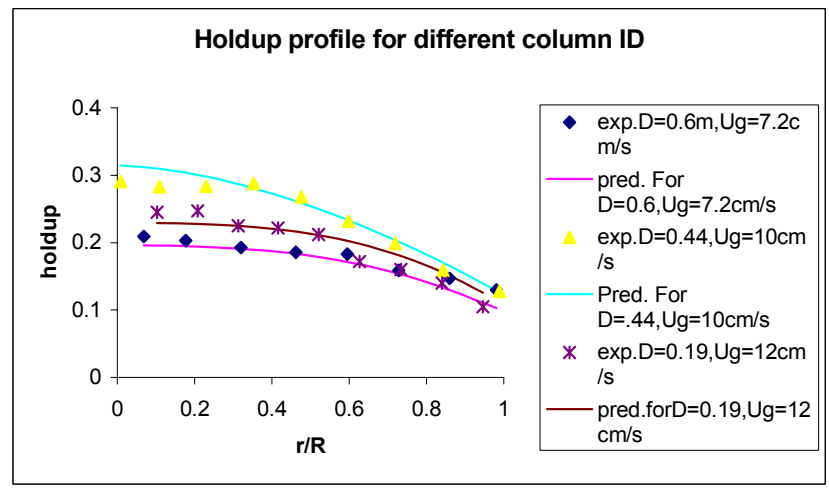

Figure 4: Effect of Column Diameter on the Holdup Profile $(\mathrm{Ug}=7.2 \mathrm{~cm} / \mathrm{s}, \mathrm{D}=0.6 \mathrm{~m}$ : Menzel et al, 1990; Ug=10cm/s, D=0.44m: Chen et al., 1998;Ug=12cm/s, D=0.19m: Kumar, 1996)

Figure 4 shows the comparison of the correlations prediction and the experimental data at different column diameters. One can see that the prediction agrees with our experimental results and literature experimental data (mean relative error is within 15\%).

\section{Summary}

It should be pointed out that in all the data presented here the cross-sectional mean holdup was known. In design situation, that would not be the case. Then a favorite correlation for the overall gas holdup can be used (Kemoun et al., 2000) to determine the mean holdup $\bar{\varepsilon}_{G}$. A correlation is proposed for prediction of radial gas hold up profiles, which are important in driving liquid recirculation in bubble column. As Figure 1-4 illustrate the agreement between the correlations predictions and the experimental data is reasonable over variable a range of operating conditions (mean relative error is less than 17\%). Further work considering gas-liquid-solid slurry system is still in progress.

\section{Notation}

c Parameter in $\mathrm{Eq}(2)$

D Column diameter, $m$

$\mathrm{Fr}_{\mathrm{g}} \quad$ Gas Froude number, dimensionless

g Acceleration due to gravity, $\mathrm{m} / \mathrm{s}^{2}$

$\mathrm{Mo}_{\mathrm{L}}$ Liquid Morton number, dimensionless

$\mathrm{n} \quad$ Parameter in Eq(1)

$\mathrm{r}, \mathrm{R} \quad$ Column radius, $\mathrm{m}$

$\mathrm{Re}_{\mathrm{G}} \quad$ Reynolds number, dimensionless

$\mathrm{U}_{\mathrm{Sg}} \quad$ Superficial gas velocity, $\mathrm{m} / \mathrm{s}$

$\mathrm{V}_{\mathrm{c}} \quad$ Liquid circulation velocity, $\mathrm{m} / \mathrm{s}$ 
Greek letters

$\varepsilon(r)$ Radial gas hold up profile

$\bar{\varepsilon}_{G} \quad$ Cross-sectional average gas hold up

$\widetilde{\varepsilon}_{G} \quad$ Radial chordal average gas holdup

$\mu_{L} \quad$ Liquid viscosity, Pa.s

$\rho_{G} \quad$ Gas density, $\mathrm{kg} / \mathrm{m}^{3}$

$\rho_{L} \quad$ Liquid density, $\mathrm{kg} / \mathrm{m}^{3}$

$\sigma_{L} \quad$ Liquid surface tension, $\mathrm{N} / \mathrm{m}$

\section{Acknowledgements}

The authors are thankful for the UCR-DOE grant (DE-FG26-99FT40594) which made this work possible. The support of CREL industrial sponsors and the Department of Energy via contract DE2295PC95051 is gratefully acknowledged as it made creation of the CREL database on holdup profiles possible.

\section{References}

Chen, J.W., Gupta, P., Sujatha, D., Al-Dahhan, M.H., Dudukovic, M.P. and Toseland, B.A.,1998, Gas Holdup Distribution in Large Diameter Bubble Columns, Flow Measurement and Inst., 9(2), 91101

Joshi, J.B., Veera, U Parasu, Parasad, Ch. V., Phanikkumar, D.V., Deshphande, N.S., Thakre, S.S. and Thorat, B.N., 1998, Gas Holdup Structure in Bubble Column Reactors PINSA 64,A, No4, 441567

Kemoun, A., Ong, B.C., Gupta, P., Al-Dahhan, M.H., \& Dudukovic, M.P.(2000). Gas holdup in bubble columns at elevated pressures via computed tomography. International Journal of Multiphase Flow (in press).

Kumar, S. B., Moslemain D. and Dudukovic, M.P., 1997 Gas Holdup Measurement in Bubble Columns Using Computed Tomography, A.I.Ch.E. Journal, 43, 1414-1425

Luo, H. and Svendsen, H.F. 1991, Turbulent Circulation in Bubble Columns from Eddy Viscosity Distributions of Single-phase Pipe Flow, Can. J. Chem.Eng., 69, 1389-1394

Menzel, Thomas, Thomas in der Weide, Staudacher, Oliver, Wein, Ondra and Onken, Ulfert, 1990, Reynolds Shear Stress for Modeling of bubble Column Reactors, Ind.\& Eng. Chem.Res., 29,988-994

Nassos, G.P. and Bankoff, S.G., 1967, Slip Velocity Ratios in An Air-water System under SteadyState and Transient Conditions, Chem. Eng. Sci. 22,667

Ueyama, K and Miyauchi, T., 1979, Properties and Recirculating Turbulent Two Phase flow in Gas Bubble Columns, AICHE J, 25,258 


\title{
Predictions of Axial Liquid Velocity Profile in Bubble Columns
}

\author{
Yuanxin Wu*, Jianhua Wen and M.H.Al-Dahhan \\ Chemical Reaction Engineering Laboratory \\ Washington University, St. Louis, MO 63130, USA
}

\section{Abstract}

The liquid flow and mixing behavior found in bubble columns are partially described by means of global parameters liquid recirculation velocity profile. Due to the complex character of the flow in bubble columns, prediction of the axial liquid circulation is still a difficult task. In this work a modified model is proposed to predict liquid recirculation profile as follows:

$\frac{V_{L}(r)}{V_{L O}}=1-2.65 * n^{0.44} * c\left[\frac{r}{R}\right]^{2.65^{*} n^{0.44} *_{c}}$

where $\mathrm{n}$ and $\mathrm{c}$ are the gas radial holdup profile parameters evaluated by the correlations proposed by $\mathrm{Wu}$, Ong and Al-Dahhan(Chemical Engineering Science, 56 (2001) 12071210).

$n=2.188 \times 10^{3} \operatorname{Re}_{G}{ }^{-0.598} \mathrm{Fr}_{g}{ }^{0.146} \mathrm{Mo}_{L}^{-0.004}$

$c=4.32 \times 10^{-2} \operatorname{Re}_{G}^{0.2492}$

The predictions of the developed liquid circulation correlation agree well with the experimental data obtained in our laboratory and reported in literature. The model is simple and is easy to use as an engineering tool to asses the liquid recirculation in bubble columns. C 2001 Elsevier Science Ltd. All rights reserved.

Key words: bubble columns, axial liquid velocity, correlation

\section{Introduction}

Bubble column reactors are widely used as gas-liquid and gas-liquid-solid contactors in much industrial aerobic fermentations, hydrogenations and other chemical operations because of their simple construction and ease of maintenance. Bubble columns combine efficient oxygen transfer and mixing with low shear forces. The behavior of these reactors is determined by their hydrodynamic properties. The complex flow and mixing behavior found in bubble columns is often described by means of global parameters such as gas holdup and liquid circulation velocity. Due to the complex character of the flow in bubble columns, their design and scale up are still a difficult task.

Many models have been proposed to analyze and predict liquid circulation. Miyauchi and Shyu(1970) and Joshi and Sharma (1979) have predicted liquid velocity in relation to the local gas hold up. However, the local gas hold up must be obtained from experimental

\footnotetext{
* Current Address: Wuhan Inst. Of Chem. Tech., Wuhan, Hubei 430073, P.R.China

Email: wyx4@public.wh.hb.cn
} 
data for both models. Zehner (1986) introduced a friction factor for liquid velocity prediction, but the value assigned by him to this parameter is not easy to justify. Kumar (1994) developed a one dimensional model which requires hold up profile and eddy viscosity or mixing length profile to which the model is found to be very sensitive. Various attempts have been made at developing functional forms for the eddy viscosity (Ueyama and Miyauchi,1979) and mixing length(Luo and Svendsen, 1991) which are required for solving the one dimensional model. However, Kumar (1994) shows that there is truly no universal expression for the mixing length or the eddy viscosity that can be successfully used under wide range of operating conditions, to predict the recirculating liquid velocity profile. In his mixing length correlation there are five parameters which are fitted to experimental data. Recently Krishna, Urseanu, van Baten and Ellenberger (1999) proposed a computational fluid dynamics based model for prediction of hold up profile and axial velocity profile. CFD based model could be a powerful design and scale-up tool after it has been fully verified. This is not yet the case.

The objective of this work is to develop a simple model based on which axial velocity profile can be predicted in relation to the gas-input rate and the column dimensions without requiring an input of the local gas hold up profiles.

\section{Model Development}

A power law liquid velocity profile is widely accepted in the literature (Montserrat T. etal.1996, Garcia-Calvo E. etal.1994). The liquid rises with the bubbles in the central portion of the column and flows downward in the outer annular section. Hence, the liquid velocity distribution in a bubble column may be expressed as

$$
\frac{V_{L}(r)}{V_{L o}}=1-2^{N / 2}\left[\frac{r}{R}\right]^{N}
$$

where $\mathrm{N}$ is the exponent of the liquid velocity profile and $\mathrm{V}_{\mathrm{LO}}$ is the liquid center line velocity. $\mathrm{N}$ varies from 2- 2.3 or higher based on different reports (Kawase and MooYoung 1986,1987 and Montserrat \& Garcia-Calvo,1996). In fact, liquid circulation is due to the existence of the gas holdup radial profile, and the radial gas hold up profile and liquid circulation are internally tied together. Both depend on superficial gas velocity, column diameter and the physical properties of the gas-liquid system investigated. The liquid according to the velocity profile of Eq. (1) is in central core region of the bubble column and flows downward in the wall zone.

A correlation of a similar form was proposed for prediction of the radial gas holdup profile (Luo \& Svendsen,1991):

$\frac{\varepsilon_{G}}{\bar{\varepsilon}_{G}}=\left(\frac{n+2}{n+2-2 C}\right)\left[1-C\left(\frac{r}{R}\right)^{n}\right]$

In equation (2), $\mathrm{n}$ is indicative of the steepness of the gas hold up profile, and $\mathrm{c}$ determines the value of holdup near the wall. It provides for the possibility for both zero 
and non-zero gas fraction values at the wall which may affect the circulation of liquid as well. Possibly exponent $\mathrm{N}$ in eq.(1) also depends on the liquid properties and on the gas flow rates (Wu, Ong \& Al-Dahhan, 2000). Hence, it may be necessary to include both $n$ and $\mathrm{c}$ in eq. (1) to predict the axial liquid velocity profile. To establish the needed relationship between the gas and liquid velocity profile, equation (1) is modified as

$$
\frac{V_{L}(r)}{V_{L o}}=1-f(n, c)\left[\frac{r}{R}\right]^{\xi(n, c)}
$$

Correlations have been developed (Wu et al., 2001) for calculation of parameters $\mathrm{n}$ and $\mathrm{c}$ as follows:

$$
\begin{aligned}
& n=2.188 \times 10^{3} \operatorname{Re}_{G}{ }^{-0.598} \mathrm{Fr}_{g}{ }^{0.146} \mathrm{Mo}_{L}^{-0.004} \\
& c=4.32 \times 10^{-2} \operatorname{Re}_{G}^{0.2492}
\end{aligned}
$$

where,

$$
\operatorname{Re}_{G}=\frac{D U_{S g}\left(\rho_{L}-\rho_{G}\right)}{\mu_{L}}, F r_{G}=\frac{U_{S g}^{2}}{g D}, M o_{L}=\frac{g \mu_{L}^{4}}{\left(\rho_{L}-\rho_{G}\right) \sigma_{L}^{3}}
$$

By fitting our computer automated radioactive particle tracking (CARPT) data, it was found that $f(n, c)=\xi(n, c)=2.65 * n^{0.44} * c$. Therefore, equation (3) becomes

$$
\frac{V_{L}(r)}{V_{L O}}=1-2.65 * n^{0.44} * c\left[\frac{r}{R}\right]^{2.65 * n^{0.44 *} *_{c}}
$$

As mentioned earlier, $\mathrm{V}_{\mathrm{Lo}}$ is the axial liquid velocity in the center of the bubble column and can be obtained from either experiments or the models reported by Zehner (1986) and Riquart (1981).

$$
\begin{aligned}
& V_{L O}=0.737\left(U_{G} D\right)^{1 / 3} \quad \text { Zehaner(1986) } \\
& V_{L O}=0.21(g D)^{1 / 2}\left(U_{G}^{3} \rho_{L} / g \mu_{L}\right)^{1 / 8} \quad \text { Riquart(1981) }
\end{aligned}
$$

From equation (4) and (5), one can see that when superficial gas velocity increases, c increases and $\mathrm{n}$ decreases. However $\mathrm{n}$ decreases with power 0.44 and $\mathrm{c}$ increases with power one, so that the overall effect is to render the axial velocity profile steeper with increased superficial gas velocity which is experimentally observed. The value of the term $\mathrm{f}(\mathrm{n}, \mathrm{c})=2.65^{*} \mathrm{n}^{0.44 * \mathrm{c}}$ is between $1.8-2.4$ with column diameter $0.1-0.63 \mathrm{~m}$ and for superficial gas velocity $0.02-0.6 \mathrm{~m} / \mathrm{s}$ and different gas-and liquid physical properties. 
A predicted liquid velocity using equation (6) with equation (4) and (5) is shown in Figure 1 and compared with experimental data. From Figure 1, it can be seen that model matches experimental data well at different superficial velocities.

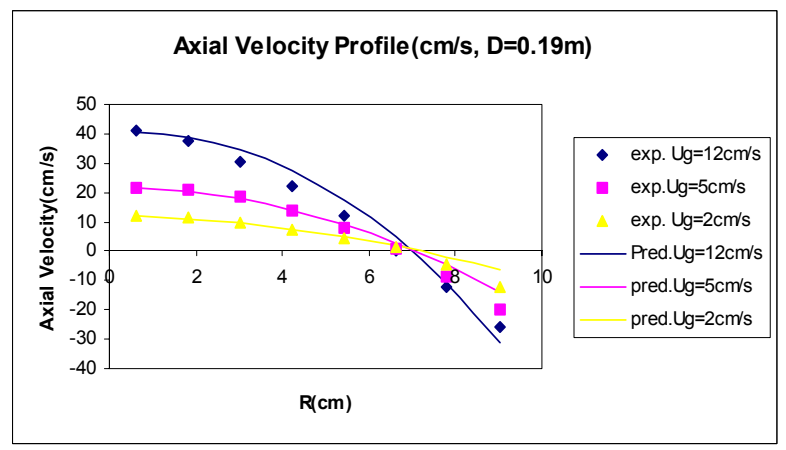

Figure 1: Comparison of model prediction and experimental data, (CARPTData for airwater system, Degaleeson, 1997)

\section{Evaluation of model predictions}

As mentioned above, equation (6) was developed by only using part of our CARPT data, and it is necessary to determine whether equation (6) can predict the experimental results from the literature to evaluate the capability of the modified correlation. We have compared the correlation predictions to the experimental data from very different sources reported in the literature and this comparison is illustrated below.

Figure 2 shows the comparison of correlation predictions and experimental data for small column diameter.

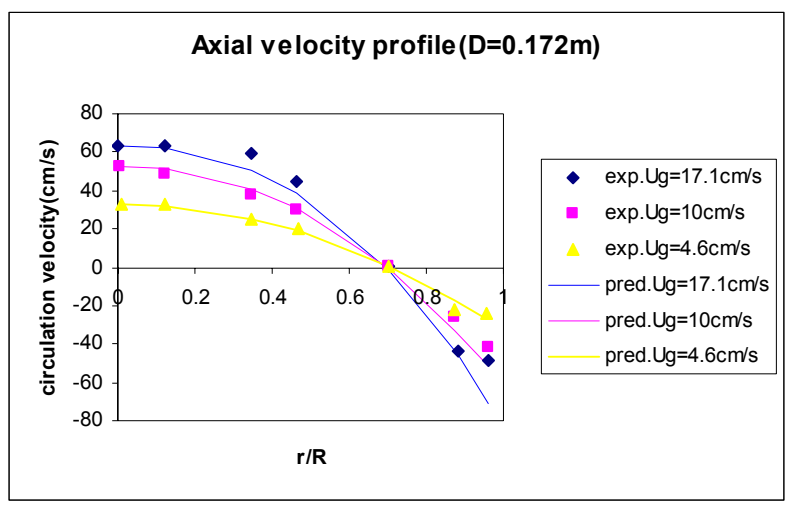

Figure 2: Comparison of model prediction and data of Pavlov (1969), Air-water system

It can been seen that for the column diameter equal to $0.172 \mathrm{~m}$, the model can predict the axial velocity profile at different superficial gas velocity with reasonable accuracy. One can clearly see that the axial liquid velocity becomes steeper with the increase in superficial gas velocity, and the model predicts the point of zero velocity well. For the 
column diameter is as big as $0.6 \mathrm{~m}$, comparison of model predictions and experimental data is plotted in Figure 3, from which it is evident that the similar predictions as that represented in Figure 2 are observed.

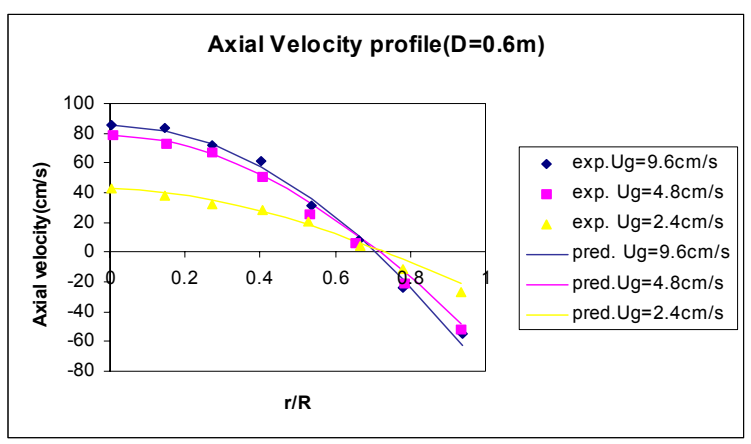

Figure3: Comparison of model prediction with the data of Menzel et al. (1990), Noncoalescence

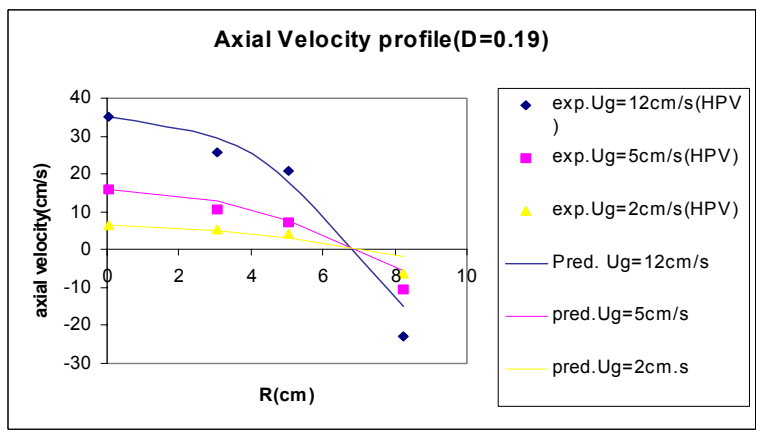

Figure 4: Comparison of model with the data from HPA (Heat Pulse Anemometry), Degaleesan, 1997

Figure 4 shows the correlation predictions of the data observed by Heat Pulse Anemometry techniques, and the comparison is good. From Figure 2-4, it is obvious that the correlation can predict the axial velocity profile of the experimental data within a range of conditions. This establishes that the proposed correlation could be used to predict axial velocity profile.

In order to compare the developed correlation with the one dimensional model (Kumar, 1994, Luo, 1991), both predictions of the proposed model and the 1D model are plotted in Figure 5 and Figure 6 for comparison with experimental data. 


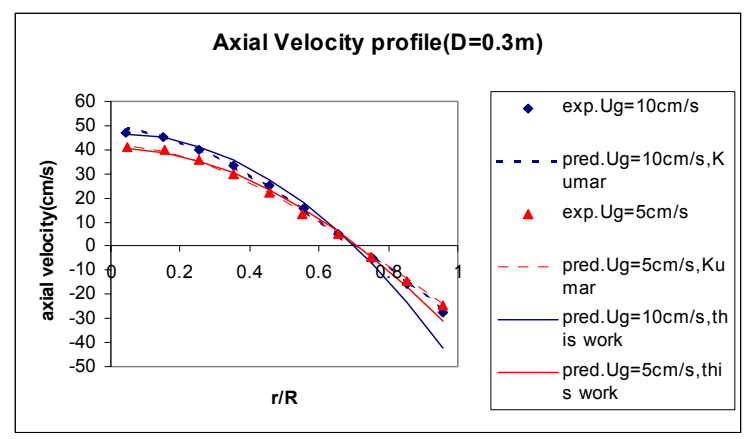

Figure 5: Comparison of this work with the CARPT data and one dimension model (Kumar 1994)

One can see that the proposed model is in reasonable agreement with the one dimensional model prediction. However, it predicts the time-averaged velocity profile at superficial gas velocity of $0.17 \mathrm{~m} / \mathrm{s}$ better than the 1D model prediction of Luo and Svendsen (1991).

It should be mentioned that the data in these figures are not used in developing the correlation. In addition, with $\mathrm{n}$ and $\mathrm{c}$ developed under pressurized conditions, it may be possible for the model to predict the axial velocity profile in bubble columns operating at pressurized conditions. But this needs to be confirmed.

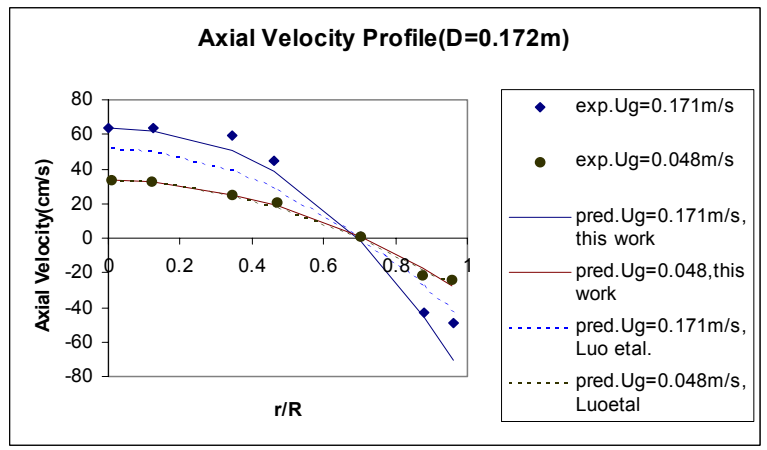

Figure 6: Comparison of this work and Luo et al. (1991) model with experimental data

\section{Summary}

The existing correlation for prediction for the axial velocity profile is modified by using the correlations for $\mathrm{n}$ and $\mathrm{c}$ developed by $\mathrm{Wu}$ et al. (2001). The modified correlation can predict the experimental data reported in the literature well within a range of conditions. The correlation is simple and is easy to use, as it requires an input only the superficial gas velocity, physical properties and column dimensions. This model can be readily used for the prediction of axial velocity profile over a range of conditions, which should help the process engineers assess convective liquid mixing in bubble column rapidly. 


\section{Nomenclature}

c Parameter in equation (2)

D Column diameter, $m$

$\mathrm{Fr}_{\mathrm{g}} \quad$ Gas Froude number, dimensionless

g Acceleration due to gravity, $\mathrm{m} / \mathrm{s}^{2}$

$\mathrm{Mo}_{\mathrm{L}}$ Liquid Morton number, dimensionless

n Parameter in equation (2)

$\mathrm{N} \quad$ Parameter in equation (1)

$\mathrm{r}, \mathrm{R}$ Column radius, $\mathrm{m}$

$\operatorname{Re}_{\mathrm{G}} \quad$ Reynolds number, dimensionless

$\mathrm{U}_{\mathrm{Sg}} \quad$ Superficial gas velocity, $\mathrm{m} / \mathrm{s}$

$\mathrm{V}_{\mathrm{c}} \quad$ Liquid circulation velocity, $\mathrm{m} / \mathrm{s}$

$\mathrm{V}_{\mathrm{L}}(\mathrm{r})$ Axial liquid velocity profile, $\mathrm{m} / \mathrm{s}$

$\mathrm{V}_{\text {Lo }} \quad$ Axial liquid velocity in the center of the column, $\mathrm{m} / \mathrm{s}$

Greek letters

$\varepsilon(r)$ Radial gas hold up profile

$\bar{\varepsilon}_{G} \quad$ Cross-sectional average gas hold up

$\widetilde{\varepsilon}_{G} \quad$ Radial average gas holdup

$\mu_{L} \quad$ Liquid viscosity, Pa.s

$\rho_{G} \quad$ Gas density, $\mathrm{kg} / \mathrm{m}^{3}$

$\rho_{L} \quad$ Liquid density, $\mathrm{kg} / \mathrm{m}^{3}$

$\sigma_{L} \quad$ Liquid surface tension, $\mathrm{N} / \mathrm{m}$

\section{Acknowledgements}

The authors are thankful for the UCR-DOE grant (DE-FG26-99FT40594) which made this work possible. The support of CREL industrial sponsors and the Department of Energy via contract DE2295PC95051 is gratefully acknowledged as it made creation of the CREL database on holdup and liquid velocity profiles possible. 


\section{References}

Degaleesan, S. (1997) Fluid dynamic measurement and modeling of liquid mixing in bubble columns, Ph.D. thesis, Washington University, St.louis, MO, USA.

Garcia-Calvo, E., and Leton, P., (1994), Prediction of fluid dynamics and liquid mixing in bubble columns, Chem. Eng. Sci., 49(21), 3643-3649.

Joshi, J.B. and Sharma, M.M., (1979), Circulation cells model for bubble columns. Trans. Inst. Chem. Eng., 57, 244-251.

Kawase, Y. and Moon-Young, M., (1986), Liquid phase mixing in bubble column with Newtonian and non-Newtonian fluid, Chem.Eng.Sci., 41,1969-1977.

Kawase, Y. and Moon-Young, M., (1987), Theoretical predictions of gas hold up in bubble column with Newtonian and non-Newtonian fluid, Ind.Eng.Chem.Res. 26,933937.

Krishna, R., Urseanu, M.I., van Baten, J.M., and Ellenberger J., (1999), Influence of scale on the hydrodynamics of bubble columns operating in the churn-turbulent regime: experiments vs. Eulerian simulations, Chem. Eng. Sci., 54(21), 4903-4911.

Kumar, S.S., (1994) Computed Tomography measurements of void fraction and modeling of the flow in bubble columns, Ph.D. Thesis, Florida Atlantic University, Boca Raton, FL, USA.

Luo H. and Svendsen H., (1991), Turbulent circulation in Bubble columns from eddy viscosity distributions of single-phase pipe flow, The Canadian Journal of Chemical Engineering, 69,1389-1394.

Menzel, T., Weide, T., Staudacher, O., Wein, O., and Onken, U., (1990), Reynolds shear stress for modeling of bubble reactors, Ind. Eng. Chem. Res., 29, 988-994.

Miyauchi, T., and Shyu, C.N. (1970), Flow of fluid in gas bubble columns, Kagaku Kogaku Ronbunshu, 34, 958-964.

Montserrat, T., and Eloy Garcia-Calvo, (1996), Prediction of hydrodynamic behavior in bubble columns, J. chem. Tech. Biotechnol. 66, 199-205.

Pavlov, V.P., (1965), Liquid circulation in a batch bubblier (in Russian) Khim. Promst. 9, 698-700

Riquarts, H.P., (1981), A physical model for axial mixing of the liquid phase for heterogeneous flow regime in bubble columns, German Chem. Eng., 4,18-23. 
Ueyama, K., \& Miyauchi, T. (1979). Properties of recirculating turbulent two-phase flow in gas bubble columns. A.I.Ch.E. Journal,25(2), 258-266.

Wu, Y., Ong B.C. \& Al-Dahhan M.H. (2001), Prediction of radial gas hold up profiles in bubble column reactors, Chemical Engineering Science, 56, 1207-1210.

Zehner, P., 1986, Momentum, mass and heat transfer in bubble columns, Part1 Flow model of the bubble column and liquid velocities, Int. Chem. Eng., 41, 1969-1977. 


\section{APPENDIX - C}

Development of An Artificial Neural Network Correlation for Prediction of Overall Gas Holdup in Bubble Column Reactors 


\title{
Development of An Artificial Neural Network Correlation for Prediction of Overall Gas Holdup in Bubble Column Reactors
}

\author{
Ashfaq Shaikh, Muthanna Al-Dahhan*
}

Chemical Reaction Engineering Laboratory, Department of Chemical Engineering, Campus Box 1198, 1 Brookings Drive, Washington University, St. Louis, MO 631304899 USA

\begin{abstract}
In the literature, several correlations have been proposed for gas holdup prediction in bubble columns. However, these correlations fail to predict gas holdup over a wide range of conditions. Based on a databank of around 3500 measurements collected from the open literature, a correlation for gas holdup was derived using a combination of Dimensional Analysis and Artificial Neural Network (ANN) modeling. The overall gas holdup was found to be a function of four dimensionless groups: $\operatorname{Re}_{\mathrm{g}}, \mathrm{Fr}_{\mathrm{g}}, \mathrm{Eo} / \mathrm{Mo}$, and
\end{abstract}


$\rho_{g} / \rho_{L}$. Statistical analysis showed that the proposed correlation has an average absolute relative error (AARE) of $15 \%$ and a standard deviation of $14 \%$. A comparison with selected correlations in the literature showed that the developed ANN correlation noticeably improved prediction of overall gas holdup. The developed correlation also shows better prediction over a wide range of operating conditions, physical properties, and column diameters, and it predicts properly the trend of the effect of the operating and design parameters on overall gas holdup.

Key Words: force analysis, artificial neural network, gas holdup, database, statistical analysis

*Corresponding author Tel.314-935-7187. E-mail muthanna@wuche.che.wustl.edu 


\section{Introduction}

Bubble columns are two-phase gas-liquid systems in which gas is dispersed through a sparger and bubbles through a liquid in vertical cylindrical columns, with or without internals. Bubble columns are widely used in chemical, petrochemical, biochemical and metallurgical industries as multiphase reactors and contactors. Examples of such chemical and petrochemical processes are partial oxidation of ethylene to acetaldehyde, wet-air oxidation (Deckwer, 1992), methanol synthesis, Fischer-Tropsch synthesis (Wender, 1996), and hydrogenation of organic liquids. In biochemical industries, bubble columns are used for cultivation of bacteria, cultivation of mold fungi (Lehmann et al., 1978), and treatment of sewage (Diesterweg, 1978). In metallurgical industries, it can be used for leaching of ores.

The advantages of bubble column include good heat and mass transfer characteristics, no moving parts and thus reduced wear and tear, higher catalyst durability, ease of operation, compactness and low operating and maintenance. Bubble columns are an attractive reactor for various multiphase processes, especially for processes involving highly exothermic reactions. These reactors are operated in semi-batch or continuous mode, with low superficial liquid velocities compared to gas velocities. For this reason, the hydrodynamics of such reactors are controlled mainly by the gas flow. In spite of the simplicity of the mechanical design of bubble columns, their fluid dynamics are complex. Therefore, due to complex interactions among the flowing phases, a proper understanding of hydrodynamics and transport parameters to enable reliable design and scale-up is still lacking.

Overall gas holdup is one of the important parameters for bubble column design and scale-up. It is defined as the fraction of the reactor dynamic volume occupied by the gas. Gas holdup and its radial profile govern liquid recirculation, flow pattern, mixing, and heat and mass transfer in bubble column reactors. Two types of regimes are encountered in bubble column operation, viz., homogeneous (bubbly) and heterogeneous (churnturbulent) flow regimes (Kastanek et al., 1993). An adequate knowledge of overall gas 
holdup and its profile are needed for flow regime identification as well as for modeling, design, and scale-up of bubble column reactors.

Over the years, overall gas holdup has been studied extensively with various measurement techniques, ranging from measuring the change in dynamic height or measuring conventional pressure drop to computed tomography. In the literature, numerous correlations have been proposed for overall gas holdup. Some of the more important correlations are listed in Table 1. Kemoun et al. (2001) compared gas holdup predicted by various correlations with the cross-sectional averaged gas holdup measured using Computed Tomography (CT) in the fully developed region at atmospheric to high pressure and at low to high superficial gas velocities. The comparison between their experimental data and predicted gas holdups from various correlations at atmospheric and high pressure $(0.7 \mathrm{MPa})$ is shown in Fig.1 and 2. The findings can be summarized as follows,

At atmospheric pressure, the correlation of Idogawa et al. (1985) gives the best agreement with the $\mathrm{CT}$ experimental data, except at $\mathrm{U}_{\mathrm{G}}=5 \mathrm{~cm} / \mathrm{s}$.

At higher pressures and over the entire superficial gas velocity range studied, the correlation of Hammer et al. (1984) gives better prediction, followed by Wilkinson et al. (1992) and Idogawa et al. (1987).

> At higher pressures and higher superficial gas velocity $\left(\mathrm{U}_{\mathrm{G}}=10 \mathrm{~cm} / \mathrm{s}\right)$, the correlation of Krishna et al. (1996) and Luo et al. (1999) also provides reasonable prediction of gas holdup.

While several correlations give reasonable predictions at different conditions, Kemoun et al. (2001) did not find any correlation that consistently predicted their experimental data at the studied operating conditions. To facilitate the scale-up of bubble columns, there is a need for a correlation that can predict overall gas holdup over a range of operating conditions, physical properties, and column dimensions.

Since the early 80 's, artificial neural networks have been used extensively in chemical engineering for such various applications as adaptive control, model based control, process monitoring, fault detection, dynamic modeling, and parameter estimation (Bhat, et al., 1990). The artificial neural network provides a nonlinear mapping between input 
and output variables and is also useful in providing cross-correlation among these variables. The mapping is performed by the use of processing elements and connection weights. The Neural Network is a useful tool in rapid predictions such as steady-state or transient process flow sheet simulations, on-line process optimization and visualization, and parameter estimation. In multiphase reactor research, there have been efforts to apply neural networks for improved prediction of design and scale-up variables. Cai et al. (1994) applied Kohonen self-organizing neural networks to identify flow regimes in horizontal air-water flow. Leib et al. (1995) used a neural network model along with the mixed-cell model to predict slurry bubble column performance for the Fischer-Tropsch synthesis. Bensetiti et al. (1997), Larachi et al. (1998), Piche et al. (2001), and Illiuta et al. (2002) used an Artificial Neural Network (ANN) to improve the prediction of various hydrodynamic parameters in packed bed and fluidized bed reactors.

Building on these studies, the focus of this work is to develop a unified correlation for overall gas holdup prediction in bubble columns which can be useful for design engineers. To develop such a correlation, an approach that combines both an Artificial Neural Network (ANN) and Dimensional Analysis has been used. The correlation has been derived from a broad experimental data bank collected from the open literature (3500 measurements covering a wide range of column dimensions and physical properties).

\section{Artificial Neural Network Modeling}

Neural Networks are computer algorithms inspired by the way information is processed in the nervous system. An Artificial Neural Network is a massively parallel distributed processor that has a natural propensity for storing experimental knowledge and making it available (Ripley, 1996). An important difference between neural networks and standard Information Technology (IT) solutions is their ability to learn. This learning property has yielded a new generation of algorithms that can

- learn from the past to predict the future 
- extract rules for reasoning in complex environments

- offer solutions when explicit algorithms and models are unavailable or too cumbersome.

Artificial Neural Networks emulates biological nervous systems and adaptive biological learning. An ANN paradigm is composed of a large number of highly interconnected processing elements, analogous to neurons, that are tied together with weighted connections that are analogous to synapses. Learning in biological systems involves adjustments to the synaptic connections between the neurons. This is true of ANNs as well. Learning typically occurs through training or exposure to a true set of input/output data where the training algorithm iteratively adjusts the connection weights. These connection weights represents the knowledge necessary to solve specific problems.

ANNs are being applied to an increasing number of real-world problems of considerable complexity. They are good pattern recognition engines and robust classifiers, with the ability to generalize in making decisions about imprecise input data. They offer ideal solutions to a variety of classification problems such as speech, character, and signal recognition, as well as prediction and system modeling where the physical processes are not understood or are highly complex. The advantage of ANNs lies in their resilience against distortions in the input data and their learning capability. They are often good at solving problems that are too complex for conventional technologies, such as problems that do not have an algorithmic solution or for which an algorithmic solution is too complex to be found.

There are multitudes of different types of ANNs. Some of the more popular include the multilayer perceptron, which is generally trained with the backpropagation of error algorithm, the Hopfield ANN, and the Kohonen ANN. Some ANNs are classified as feedforward, while others are recurrent, depending on how data is processed through the network. Another way of classifying ANN types is by their method of learning, as some ANNs employ supervised training, while others are referred to as unsupervised or selforganizing (Ripley, 1996). 
In this work, a multilayer neural network has been used, as it is effective in finding complex non-linear relationships. It has been reported that multilayer ANN models with only one hidden layer are universal approximators (Hornik et al., 1989). Hence, a threelayer feedforward neural network is chosen as a regression model. The weighting coefficients of the neural network are calculated using the special-purpose software NNFit (Cloutier et al.,1996). NNFit is a non-linear regression software that discloses relationships between a set of normalized input variables, $U_{i}$, and a set of normalized output variable, $\mathrm{S}_{\mathrm{k}}$. Figure 3 shows the transformation $\mathrm{S}=f(\mathrm{U})$ using a neural network with a single hidden layer. The transformation of actual variables $(\mathrm{X}, \mathrm{Y})$ to normalized variables (U, S) is given by (Cloutier et al., 1996),

$U_{i}=\frac{\log \left(X_{i} / X_{\text {min }}\right)}{\log \left(X_{\text {max }} / X_{\text {min }}\right)}$

$$
S_{k}=\frac{\log \left(Y_{k} / Y_{\min }\right)}{\log \left(Y_{\max } / Y_{\min }\right)}
$$

where, $X_{i}$ and $Y_{k}$ are raw input and output variables. The basic structure of this type of neural network is described by the following set of equations. The various layers are interconnected to each other by a sigmoid function through the fitted parameters $w_{i j}$, $w_{j k}$ in the following manner,

$$
S_{k}=\frac{1}{1+\exp \left[-\sum_{j=1}^{J+1} w_{j k} H_{j}\right]}
$$

and 


$$
H_{j}=\frac{1}{1+\exp \left[-\sum_{i=1}^{I+1} w_{i j} U_{i}\right]}
$$

where $\mathrm{I}, \mathrm{J}, \mathrm{K}$ indicate the input, hidden and output nodes of the ANN structure, respectively. $\mathrm{H}_{\mathrm{J}+1}$ and $\mathrm{U}_{\mathrm{I}+1}$ (Figure 3 ) are the bias constants which are set equal to one. $w_{i j}$ and $w_{j k}$ are weighting parameters which are fitted by the NNFit regression model, using a quadratic criterion as a minimization algorithm, a quasi-Newton method of the BGFS type (Broyden-Fletcher-Goldfrab-Shanno) (Cloutier et al., 1996).

\section{Development of the ANN based correlation}

The development of the ANN-based correlation began with the collection of a large databank. The physical parameters were then subjected to force analysis in order to maintain dimensional homogeneity. The last step was to perform a neural regression, and to validate it statistically.

\section{Collection of data}

As mentioned earlier, over the years researchers have amply quantified the hydrodynamics of bubble column reactors based on the overall gas holdup. In this work, about 3500 experimental points have been collected from 60 sources spanning the years 1965 to 2000. This wide range of database includes experimental information from different physical systems to provide a unified correlation for overall gas holdup. Table 2 suggests the wide range of the collected databank for gas holdup.

Most of the hydrodynamic studies on bubble columns were performed using air-water systems. Hence, the majority of the current database (around 45\%) comprises overall gas holdup data from air-water studies. To assess the impact of physical properties such as density, surface tension, and viscosity, several other gas-liquid systems were included in the database. However, in this work we have included only pure liquids systems (approximately 30 different liquids). Recently, Syeda et al. has developed a correlation, based on their own experimental data, to predict gas holdup in bubble column reactors 
with pure as well as binary liquids. Bubble columns are generally operated with low liquid velocities, which have been reported to have little or no effect on overall gas holdup (Kelkar et al., 1983, Shetty et al., 1992). Hence, in this work we have considered data only for columns with liquid in batch mode and gas in continuous mode. As industrial conditions of interest are at high pressure, we have added the available experimental studies at high pressures up to $2 \mathrm{MPa}$. Since reactor scale-up extends small diameter behavior to large diameters, and in order to make the developed correlation industrially useful, we have included data obtained up to $5.5 \mathrm{~m}$ column diameter, the largest diameter described in the open literature. All the data was collected for cylindrical columns, as they are the favored geometry in a majority of industrial applications. There are different spargers used in various studies. We have mentioned only the most commonly used spargers in Table 2. However, in the most literature studies the mentioned spargers have been used with some modifications in geometry or by changing number of holes or hole sizes. Since the data was collected from wide range of sources, there is no uniformity in the measurement techniques of gas holdup. The techniques range from measurement by level change, or pressure drop up to densitometry and computed tomography.

\section{Force Analysis}

The force analysis checked whether the physical parameters in the database can be formulated in a dimensionally homogeneous manner or not. It consists of two steps

i) All physical parameters that influence overall gas holdup are put in a so called "wish-list".

ii) The dimensional homogeneity of the physical parameters was checked by transforming them into various forces.

Based on the extensive literature review, the following input variables have been found to affect gas holdup 
i) Superficial gas velocity: Gas holdup increases with an increase in superficial gas velocity. The effect is relatively weaker in the churn-turbulent regime.

ii) Column Diameter: Gas holdup decreases with an increase in column diameter. Many researchers have claimed that above $15 \mathrm{~cm}$, the effect of column diameter is negligible (Botton, 1978, Wilkinson, 1991).

iii) Operating Pressure: An increase in pressure increases gas density and decreases the mean bubble size and the population of large bubbles, thus increasing gas holdup (Wilkinson 1991, Smith et al., 1995).

iv) Liquid physical properties: An increase in density and a decrease in surface tension and viscosity both increase gas holdup (Wilkinson, 1991).

v) Sparger Design: The effect of the sparger is influential in the homogeneous regime (Kelkar et al., 1983).

Once the crucial identification of raw variables has been performed, the input variables were then converted into various physical forces. Some of the important forces are
a) gas inertial force: $\rho_{G} u_{G}^{2}$
b) gas viscous force: $\mu_{G} u_{G} / d$
c) liquid gravitational force: $\rho_{L} g d$
d) gas gravitational force: $\rho_{G} g d$
e) capillary force: $\sigma_{L} / d$

Dimensionless numbers were then formed by taking ratios of various physical forces which are determined from the input variables. In addition to this, the various dimensionless groups used in the gas holdup correlations reported in literature were considered. Then, on the basis of the observed effect of some parameters on the overall gas holdup, some of the dimensionless groups such as ratios of densities, etc., were added. 
The main advantage of performing dimensional analysis is to reduce the number of input parameters, i.e., there are fewer dimensionless input groups than the raw parameters. The other advantage of dimensional analysis lies in the "scale-invariant" property of a dimensionless frame. The "scale-invariance" makes dimensional analysis a primary step in scale-up of reactors (Zlokarnik, 1998).

\section{Neural Regression}

Force analysis is used to produce dimensionless groups in this case, but it alone can not determine which groups are relevant and should be used as input. Therefore, we used the following methodology to select the most pertinent inputs (Bensetiti et al., 1997).

Out of the number of dimensionless groups derived, we used ANN regression to establish the best set of chosen dimensionless groups, which describes overall gas holdup (Bensetiti et al., 1997, Larachi et al., 1998). The following criteria guide the choice of the set of input dimensionless groups:

- The dimensionless groups should be as few as possible,

- Each group should be highly cross-correlated to the output parameter,

- These input groups should be weakly cross-correlated to each other,

- The selected input set should give the best output prediction, which is checked by using statistical analysis [e.g., average absolute relative error (AARE), standard deviation, cross-correlation coefficient].

- There should be minimum complexity in neural network architecture, i.e., a minimum number of hidden layers $\mathrm{J}$.

While choosing the most expressive dimensionless groups, there is a compromise between the number of dimensionless groups and prediction. The main concern with the number of dimensionless groups is due to two reasons: first, there should be fewer expressive groups than raw parameters, and second for feasible scale-up we may need a minimum number of dimensionless groups. 
The cross-correlation analysis which signifies the strength of the linear relation between input and output is then used to find the dependence between input and output groups. A number of inputs can be highly cross-correlated to output, but there should not be any dependency between these groups; otherwise, it just adds to the complexity of the structure rather than contributing significantly to improve the quality of the network. One should be careful here: although the cross-correlation analysis reveals the dependence between inputs and outputs, it also hides non-monotonic relationships. This can result into losing an important dimensionless group. Therefore in this study, several sets of input groups were made and tested via rigorous trial-and-error on the Artificial Neural Network. The above mentioned criteria were then used to identify the most pertinent set of input groups.

The statistical analysis of prediction is based on the following criteria:

- The average absolute relative error (AARE) should be minimum.

$$
A A R E=\frac{1}{N} \sum_{1}^{N}\left|\frac{y_{\text {predicted }}-y_{\text {experimental }}}{y_{\text {experimental }}}\right|
$$

- The standard deviation should be minimum.

$$
\sigma=\sqrt{\sum_{i=1}^{N} \frac{\left(\left|\frac{y_{\text {predictedl }(i)}-y_{\text {experimental }(i)} \mid}{y_{\text {experimental }(i)}}\right|-A A R E\right)^{2}}{N-1}}
$$

- The cross-correlation coefficient, $\mathrm{R}$ between input and output should be around unity 


$$
R=\frac{\sum_{i=1}^{N}\left(y_{\text {experimental }(i)}-y_{\text {experimental(mean })}\right)\left(y_{\text {predicted }(i)}-y_{\text {predicted(mean })}\right)}{\sqrt{\sum_{i=1}^{N}\left(y_{\text {exp erimental }(i)}-y_{\text {exp erimental (mean })}\right)^{2}} \sqrt{\sum_{i=1}^{N}\left(y_{\text {predicted }(i)}-y_{\text {predicted (mean })}\right)^{2}}}
$$

Neural networks often encounter the well known 'overfitting' problem, which can make use of the ANN unreliable. To avoid 'overfitting' and make the ANN more useful, the following approach was used. The whole database was split into two parts, learning and generalization. The first part, called the 'learning file', was used to perform minimization using the ANN. The remaining part, called the 'generalization file', was used to validate the model. Following the common practice, the learning file was made by randomly selecting about $70 \%$ of the database to train the network. The remaining $30 \%$ of data was then used to check the generalization capability of the model. The hidden layers, J, and fitting parameters $w_{i j}$ and $w_{j k}$ are a priori unknown. The number of hidden layers was varied and choosen empirically according to the above criteria. The weighting parameters were then determined by non-linear least-square regression over known random inputs/outputs ( $70 \%$ of the data, which was picked randomly). The remaining $30 \%$ of the database was utilized for validation of predicted weighting parameters. The chosen set of inputs must show the best prediction during training and generalization, i.e., show the least error on both learning and generalization files.

\section{Results}

After collecting the large databank, we subjected it to dimensional analysis, which resulted into hundreds of dimensionless groups. As a matter of fact, using all these groups is not feasible. Hence, to make the use of the developed model feasible, after forming a number of sets of dimensionless groups, cross-correlation analysis was performed. As the cross-correlation analysis can hide non-monotic relationships, rigorous trial-and-error testing with the aid of ANN was also performed. The criteria mentioned above led to four

pertinent input dimensionless groups: $\operatorname{Re}_{\mathrm{g},} \mathrm{Fr}_{\mathrm{g}}$, Eo/Mo, and $\rho_{g} / \rho_{L}$. The ratio_of the 
densities of the gas and liquid was added to account for the effect of high pressure. This particular set of dimensionless groups showed consistent performance on both the learning and generalization file. The sets of dimensionless groups which did not show consistent performance were omitted, despite their remarkable performance on the learning file. Table 3 lists the most expressive input groups and is accompanied by the set of equations and weighting parameters. To use the ANN correlation, these equations and parameters can be readily put in a spreadsheet file for overall gas holdup calculations in bubble column reactors. They will also be available later on our website (http://crelonweb.che.wustl.edu).

Figure 4 shows the parity plot of the experimental and predicted overall gas holdup using the ANN correlation on the whole database. The ANN predicts the overall gas holdup with an AARE of $15 \%$. For comparison, Figure 5 is the parity plot of the experimental and predicted overall gas holdup, based on the whole database and using selected literature correlations along with the ANN correlation. In this case, the correlations were selected based on the conclusions of Kemoun et al., (2001). From the figure, it is clear that the ANN correlation predicts overall gas holdup better than these two correlations. Moreover, Table 4 compares an additional important correlations in literature on the basis of statistical analysis, and confirms that the ANN performs better than they do.

Table 5 shows the statistical parameters for some of the input sets of the ANN considered in this analysis. It includes different numbers of hidden layers to justify the selection of the current input set.

\section{ANN correlation prediction of gas holdup using different liquids}

The major portion of the databank consists of water as the liquid phase, since most of the reported studies used water for simplicity and economy. As mentioned earlier, the databank has a wide range of fluid physical properties, therefore we have performed statistical analysis on fluids with different physical properties to check whether the ANN correlation predicts overall gas holdup consistently or not. Figure 6 shows the parity plot 
of the experimental and predicted gas holdup using the ANN correlation for water at different operating conditions, while Table 6 shows the statistical analysis of the gas holdup predictions for different fluids, along with their physical properties. It is obvious that the ANN correlation predicts satisfactorily the effect of liquid physical properties on the overall gas holdup.

\section{ANN correlation prediction of gas holdup at different pressures}

In the literature, there are many correlations proposed for prediction of overall gas holdup at atmospheric pressure, and there are some correlations developed at high pressure as well. As mentioned by Kemoun et al., (2001), some of these correlations show good prediction of overall gas holdup at atmospheric pressure but fail at high pressures. Although we have included data up to $2 \mathrm{MPa}$, the major part of the databank is of gas holdup at atmospheric pressures. Therefore, we have separated data of different pressures and performed statistical analysis to check how well the ANN predicts gas holdup at varied pressures. Such statistical evaluation is shown in Table 7, and it is obvious that the

developed ANN correlation predicts well the overall gas holdup at both elevated and atmospheric pressures.

\section{ANN correlation prediction of gas holdup for different column diameters}

The agreement between predicted and experimental gas holdup for different column diameters has been evaluated by the statistical analysis shown in Table 8. The ANN consistently predicts gas holdup over a wide range of diameters within acceptable error.

\section{ANN Correlation Prediction of the Trend of the Effect of Different Parameters}

In this part of the work, we checked how well the prediction of the developed ANN correlation captures the reported trend of the effect of different parameters on the overall gas holdup. 


\section{Effect of Column Diameter}

Figure 7 shows a comparison between the predictions obtained using the ANN correlation and experimental data for air-water systems at ambient conditions in different columns $(\mathrm{d}=0.01,0.15,0.3 \mathrm{~m})$ at different superficial gas velocities. The trend shown by the ANN correlation is in agreement with published literature.

\section{Effect of Operating Pressure}

Figure 8 shows a comparison between the predictions obtained using the ANN correlation and experimental data for a air-water system in a $0.15 \mathrm{~m}$ diameter column at different operating pressures $(\mathrm{P}=0.1,0.6,1.2 \mathrm{MPa})$. It shows an increase in gas holdup with an increase in pressure, as reported in the literature (Wilkinson 1991).

\section{Effect of Liquid Physical Properties}

To check the effect of liquid properties, ANN correlation simulations were carried out at the experimental conditions of Reilly et al. (1994) and Vermeer et al. (1981), with the results shown in Figure 9. This particular data from Reilly et al. (1994) and Vermeer et al. (1981) was not included in the database used for training and validation of the developed ANN correlation.

To compare the predictions with the Reilly et al. (1994), a simulation was carried out at ambient conditions with ISOPAR G* $\left(\rho_{\mathrm{L}}=740 \mathrm{~kg} \cdot \mathrm{m}^{-3}, \mu_{\mathrm{L}}=0.861 \mathrm{mPa} . \mathrm{s}, \sigma_{\mathrm{L}}=0.0235\right.$ N.m $\left.{ }^{-1}\right)-\mathrm{CO}_{2}\left(\rho_{\mathrm{G}}=1.84 \mathrm{~kg} \cdot \mathrm{m}^{-3}\right)$ in $15 \mathrm{~cm}$ diameter column. Similarly, to compare the predictions with the Vermeer et al. (1981), a simulation was carried out at ambient conditions in $19 \mathrm{~cm}$ column with Turpentine $\left(\rho_{\mathrm{L}}=761 \mathrm{~kg} \cdot \mathrm{m}^{-3}, \mu_{\mathrm{L}}=0.00094\right.$ Pa.s, $\sigma_{\mathrm{L}}=$ $\left.0.024 \mathrm{~N} . \mathrm{m}^{-1}\right)-\mathrm{N}_{2}\left(\rho_{\mathrm{G}}=1.146 \mathrm{~kg} \cdot \mathrm{m}^{-3}\right)$. The predictions are in good agreement with the experimental data, particularly in the bubbly flow regime $\left(u_{G}<3 \mathrm{~cm} / \mathrm{s}\right)$. However, in the transition region $\left(u_{G}=3-5 \mathrm{~cm} / \mathrm{s}\right)$ and churn-turbulent flow regime $\left(u_{G} \geq 5 \mathrm{~cm} / \mathrm{s}\right)$, there is some deviation between the experimental data of Reilly et al. (1994) and the ANN correlation prediction, although the difference is within 13\% AARE. This agreement 
suggests that grouping the data for different flow regime and having a correlation for each flow regimes would benefit the accuracy of the prediction. This approach is being considered for future evaluation.

Overall, the simulations performed using the ANN correlation predict the effect of different parameters on overall gas holdup per the trend reported in the literature. They prove its utility as a design estimation tool for bubble column reactors.

\section{Conclusions}

Compared to the selected literature correlations, the Artificial Neural Network correlation shows noticeable improvement in the prediction of overall gas holdup. The neural network correlation yields an AARE of $15 \%$, with a standard deviation of $14 \%$, which is better than those obtained for the selected literature correlations. This work identified $\mathrm{Re}_{\mathrm{g},} \mathrm{Fr}_{\mathrm{g}}, \mathrm{Eo} / \mathrm{Mo}$, and $\rho_{g} / \rho_{L}$ as expressive dimensionless groups to predict overall gas holdup. Also, the ANN correlation yielded improved predictions for a variety of liquids, a wide range of operating pressures, and various column diameters. In addition, the developed correlation captures properly the trend of the effect of various operating and design parameters on the overall gas holdup reported in the literature. Hence the developed ANN correlation should be useful in the scale-up of bubble column reactors.

\section{Acknowledgements}

The authors are thankful for the UCR-DOE grant (DE-FG-26-99FT40594) which made this work possible. The authors also gratefully acknowledge the help extended by David Newton (an undergraduate student) during the data collection. The encouragement and the support received from Prof. M. P. Duduckovic' during the course of this work have been invaluable. 


\section{Nomenclature}

\begin{tabular}{ll}
$\mathrm{d}$ & column diameter, $\mathrm{m}$ \\
$\mathrm{D}_{\mathrm{R}}$ & ratio of gas and liquid phase densities, dimensionless \\
$\mathrm{Eo}$ & Etovos number, dimensionless \\
$\mathrm{Fr}_{\mathrm{g}}$ & gas Froude number, dimensionless \\
$\mathrm{g}$ & gravitational constant, $\mathrm{m} \mathrm{s}^{-2}$ \\
$\mathrm{I}$ & number of input nodes \\
$\mathrm{J}$ & number of hidden layers \\
$\mathrm{K}$ & number of output layers \\
$\mathrm{Mo}$ & liquid Morton number, dimensionless \\
$\mathrm{N}$ & number of data points \\
$\mathrm{P}$ & operating pressure, atm \\
$\mathrm{R}$ & cross-correlation coefficient \\
$\mathrm{S}_{\mathrm{k}}$ & normalized output variable \\
$\mathrm{u}_{\mathrm{g}}$ & superficial gas velocity, $\mathrm{m} \mathrm{s}^{-1}$ \\
$\mathrm{u}_{\mathrm{g}, \text { trans }}$ & transition velocity, m s \\
$\mathrm{u}_{\mathrm{s}, \mathrm{b}}$ & small bubble velocity, $\mathrm{m} \mathrm{s}^{-1}$ \\
$\mathrm{u}_{1, \mathrm{~b}}$ & large bubble velocity, $\mathrm{m} \mathrm{s}^{-1}$ \\
$\mathrm{U}_{\mathrm{i}}$ & normalized input variable \\
$w_{i j}, w_{j k}$ & ANN fitting parameters \\
\hline
\end{tabular}

Greek letters

$\begin{array}{ll}\sigma & \text { standard deviation } \\ \rho_{\mathrm{g}} & \text { gas phase density, } \mathrm{kg} \mathrm{m}^{-3} \\ \rho_{\mathrm{L}} & \text { liquid phase density, } \mathrm{kg} \mathrm{m}^{-3} \\ \sigma_{\mathrm{L}} & \text { liquid surface tension, } \mathrm{N} \mathrm{m}^{-1} \\ \mu_{\mathrm{L}} & \text { liquid viscosity, } \mathrm{kg} \mathrm{m}^{-1} \mathrm{~s}^{-1} \\ \varepsilon_{\mathrm{b}} & \text { gas holdup of small bubbles }\end{array}$


$\begin{array}{ll}\varepsilon_{\mathrm{G}, \text { trans }} & \text { gas holdup at transition point } \\ \varepsilon_{\mathrm{G}} & \text { overall gas holdup, dimensionless }\end{array}$

Abbrevations

AARE average absolute relative error

ANN artificial neural network

CT computed tomography 


\section{References}

Bensetiti, Z., Larachi, F., Grandjean, B.P.A., and Wild, G., (1997), Liquid saturation in cocurrent upflow fixed-bed reactors: A state-of-the-art-correlation, Chem Eng Sci, 52 $(21 / 22), 4239$.

Bhat, N., McAvoy, T. J., Use of neural nets for dynamic modeling and control of chemical process systems, Comput. Chem. Eng. (1990), 14(4-5), 573-83.

Botton, R.; Cosserat, D., Charpentier, J. C., (1978) Influence of column diameter and high gas throughputs on the operation of a bubble column, Chem. Eng. J. (Lausanne), 16(2), 107.

Cai, Shiqian; Toral, Haluk; Qiu, Jianhung, Archer, John S., (1994) Neural network based objective flow regime identification in air-water two phase flow, Can J Chem Eng, 72(3), 440.

Cloutier, P., Tibirna, C., Grandjean, B.P.A., and Thibault, J., (1996), NNFit, non-linear regression program based on multilayered neural network models. www.gch.ulaval.ca/ nnfit/

Deckwer, W., Bubble Column Reactors, John Wiley \& Sons, 1992.

Diesterwerg, G., Fuhr, H., and Reher, P., (1978), Die Bayer-Turmbiologie, Industrieabwasser, 7.

Hornik, K., Stinchcombe, M., and White H., (1989), Multilayer feedforward neural networks are universal approximators. Neural Networks, 2, 359. 
Iliuta, I., Grandjean, B. P. A., Larachi, F., (2002) Hydrodynamics of trickle-flow reactors: updated slip functions for the slit models, Chemical Engineering Research and Design 80(A2), 195.

Kastanek, F., Zahradnik, J., Kratochvil, J., and Cermak, J., (1983) Chemical Reactions for Gas-Liquid Systems, Ellis Horwood, New York.

Kelkar, B.G., Phulgaonkar, S.R., Shah, Y.T., (1983), The Effect of Electrolyte Solutions on Hydrodynamic and Backmixing Characteristics in Bubble Columns, Chem Engng J, $27,125$.

Kemoun, A., Ong, B.C., Gupta, P., Al-Dahhan, M.H., \& Dudukovic, M. P., (2001), Gas holdup in bubble columns at elevated pressures via computed tomography. International Journal of Multiphase Flow, 27, 929.

Larachi, F., Bensetiti, Z., Grandjean, B.P.A., and Wild, G., (1998), Two-phase frictional pressure drop in flooded-bed reactors: A state-of-the-art-correlation, Chem Eng Technol, $21,887$.

Leib, T.M., Mills P.L., Lerou J.J., Turner J.J., (1995), Evaluation of Neural Networks for Simulation of Three-Phase Bubble Column Reactors, Trans IChemE, 73, Part A, 690.

Lehman, J. and Hammer, J., (1978), Continuous fermentation in tower fermentor, I European congress on biotechnology, Interlaken, Part 1, 1.

Piche, S., Larachi, F., Grandjean, B. P. A., (2001) Improved liquid hold-up correlation for randomly packed towers, Chemical Engineering Research and Design, 79(A1), 71.

Reilly, I. G., Scott, D. S., De Bruijn, T. J. W., MacIntyre, D., (1994) The role of gas phase momentum in determining gas holdup and hydrodynamic flow regimes in bubble column operations, Can J Chem Eng, 72(1), 3. 
Ripley, B.D. (1996) Pattern Recognition and Neural Networks, Cambridge: Cambridge University Press.

Smith, G. B., Gamblin, B. R.; Newton, D., (1995), X-ray imaging of slurry bubble column reactors: the effects of system pressure and scale, Chem. Eng. Res. Des. (1995), 73 (A6), 632.

Shetty, S. A., Kantak, M. V., Kelkar, B. G., (1992) Gas-phase backmixing in bubblecolumn reactors. AIChE J 38(7), 1013.

Syeda, Sultana R., Afacan, Artin, Chuang, Karl T., (2002) Prediction of gas hold-up in a bubble column filled with pure and binary liquids. Canadian Journal of Chemical Engineering, 80(1), 44.

Vermeer, Derk J., Krishna, Rajamani, (1981) Hydrodynamics and mass transfer in bubble columns in operating in the churn-turbulent regime, Ind Eng Chem Process Des Dev, 20(3), 475 .

Wender, I., Reactions of Synthesis Gas, (1996) Fuel Processing Technology, 48, 189.

Wilkinson, P.M., Physical Aspects and Scale-up of High Pressure Bubble Columns, Ph.D. Thesis, (1991), University of Groningen.

Zlokarnik, M., (1998), Problems in the application of dimensional analysis and scale-up of mixing operations, Chem Eng Sci, 53 (17), 3023

Idogawa, K., Ikeda, K., Fukuda, T., Morooka, S., (1985) Effect of gas and liquid properties on the behavior of bubbles in a bubble column under high pressure, Kag. Kog. Ronb. 11, 432

Idogawa, K., Ikeda, K., Fukuda, T., Morooka, S., (1987) Effect of gas and liquid properties on the behavior of bubbles in a bubble column under high pressure, Int. Chem. Eng. 27, 93. 
Krishna, R., Ellenberger, J., (1996) Gas holdup in bubble column reactors operating in the churn turbulent flow regime, AIChE J. 42, 2627

Luo, X., Lee, D.J., Lau, R., Yang, G., Fan, L.S., (1999) Maximum stable bubble size and gas holdup in high pressure slurry bubble columns, AIChE J. 42, 665

Wilkinson, P.M., Spek, A.P., Van Dierendonck, L.L., (1992) Design parameters estimation for scale-up of high pressure bubble columns, AIChE J. 38, 544 


\section{Tables}

Table 1 Summary of Overall Gas Holdup Correlations

Table 2 Range of column dimensions, physical properties, operating pressures and type of spargers included in the collected databank

Table 3 Set of equations and fitting parameters for the neural network correlation

Table 4 Comparison of ANN and previous literature correlations

Table 5 Error analysis for some of the input groups at various $\mathrm{J}$ values

Table 6 Statistical analysis for different liquids using ANN correlation

Table 7 Statistical analysis at different pressures using ANN correlation

Table 8 Statistical analysis for different column diameters using ANN correlation 
Table 1: Summary of Overall Gas Holdup Correlations

Researcher Correlations

Akita and

Yoshida

(1973)

$$
\frac{\varepsilon_{G}}{\left(1-\varepsilon_{G}\right)^{4}}=0.2\left(\frac{g d^{2} \rho_{L}}{\sigma_{L}}\right)^{0.125}\left(\frac{g d^{3}}{\mu_{L}}\right)^{0.083}\left(\frac{u_{G}}{(g d)^{0.5}}\right)
$$

Hikita et al.

$$
\varepsilon_{G}=0.672\left(\frac{u_{G} \mu_{L}}{\sigma_{L}}\right)\left(\frac{\mu_{L}^{4} g}{\rho_{L} \sigma_{L}^{3}}\right)^{-0.131}\left(\frac{\rho_{G}}{\rho_{L}}\right)^{0.062}\left(\frac{\mu_{G}}{\mu_{L}}\right)^{0.107}
$$

Hammer $e t$

al. (1984)

$$
\frac{\varepsilon_{G}}{\left(1-\varepsilon_{G}\right)}=0.4\left(\frac{u_{G} \mu_{L}}{\sigma_{L}}\right)^{0.87}\left(\frac{\mu_{L}^{4} g}{\rho_{L} \sigma_{L}^{3}}\right)^{-0.27}\left(\frac{\rho_{G}}{\rho_{L}}\right)^{0.17}
$$

Idogawa et

al. (1985)

$$
\frac{\varepsilon_{G}}{\left(1-\varepsilon_{G}\right)}=1.44 u_{G}^{0.58} \rho_{G}^{0.12} \sigma_{L}^{-0.16 \exp (-P)}
$$

Reilly et al. (1986)

$$
\varepsilon_{G}=296 u_{G}^{0.44} \rho_{L}^{-0.98} \rho_{G}^{0.19} \sigma_{L}^{-0.16}+0.009
$$

Idogawa et al. (1987)

$$
\frac{\varepsilon_{G}}{\left(1-\varepsilon_{G}\right)}=0.059 u_{G}^{0.8} \rho_{G}^{0.17}\left(\frac{\sigma_{L}}{72}\right)^{-0.22 \exp (-P)}
$$

Dharwadkar et al. (1987)

Wilkinson et al. (1992)

$$
\varepsilon_{G}=0.07 u_{G}^{0.5} \mu_{L}^{-0.04} \sigma_{L}^{-0.75}
$$

$$
\begin{aligned}
& u_{G}<u_{\text {trans }} \varepsilon_{G}=\frac{u_{G}}{u_{s, b}} \\
& u_{G}>u_{\text {trans }} \varepsilon_{G}=\frac{u_{\text {trans }}}{u_{s, b}}+\frac{u_{G}-u_{\text {trans }}}{u_{l, b}}
\end{aligned}
$$

where, $\frac{u_{\text {trans }}}{u_{s, b}}=0.5 \exp \left(-193 \rho_{G}^{-0.61} \mu_{L}^{0.5} \sigma_{L}^{0.11}\right)$

$$
\begin{aligned}
& \frac{\mu_{L} u_{s, b}}{\sigma_{L}}=2.25\left(\frac{\sigma_{L}^{3} \rho_{L}}{g \mu_{L}^{4}}\right)^{-0.273}\left(\frac{\rho_{L}}{\rho_{G}}\right)^{0.077} \\
& \frac{\mu_{L} u_{l, b}}{\sigma_{L}}=\frac{\mu_{L} u_{s, b}}{\sigma_{L}}+2.4\left\{\frac{\mu_{L}\left(u_{G}-u_{\text {trans }}\right.}{\sigma_{L}}\right\}^{0.757}\left(\frac{\sigma_{L}^{3} \rho_{L}}{g \mu_{L}^{4}}\right)^{-0.077}\left(\frac{\rho_{L}}{\rho_{G}}\right)^{0.077}
\end{aligned}
$$




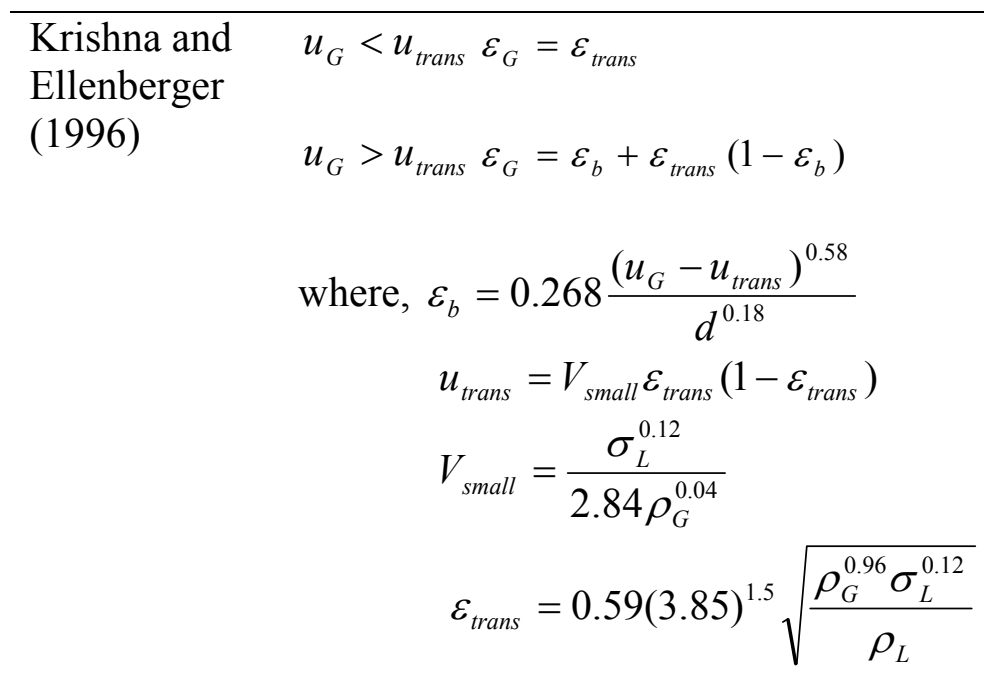

Kojima et al. (1997)

$$
\varepsilon_{G}=1.18 u_{G}^{0.679}\left(\frac{\sigma_{L}}{\sigma_{L, 0}}\right)^{-0.546} \exp \left\{1.27 \times 10^{-4}\left(\frac{\rho_{L} Q^{2}}{d_{0}^{3} \sigma_{L}}\right)\left(\frac{P}{P_{o}}\right)\right\}
$$

Joshi et al. (1998)

$$
\varepsilon_{G}=0.62 u_{G}^{0.56}\left(\frac{72}{\sigma_{L}}\right)^{0.15}\left(\frac{1}{\mu_{L}}\right)^{0.15}\left(\frac{\rho_{G}}{1.3}\right)^{0.15}\left(\frac{1000}{\rho_{L}}\right)^{0.15}
$$

Luo et al.

(1999)

$$
\begin{gathered}
\frac{\varepsilon_{G}}{\left(1-\varepsilon_{G}\right)}=\frac{2.9\left(\frac{u_{G}^{4} \rho_{G}}{\sigma_{L} g}\right)^{\alpha}\left(\frac{\rho_{G}}{\rho_{L}}\right)^{\beta}}{\left[\cosh \left(M o_{L}^{0.054}\right)\right]^{0.41}} \\
\alpha=0.21 M o_{L}^{0.0079} ; \beta=0.096 M o_{L}^{-0.011}
\end{gathered}
$$

Jordan et al. (2001)

$$
\frac{\varepsilon_{G}}{\left(1-\varepsilon_{G}\right)}=b_{1}\left(\frac{g \rho_{L} d_{B}^{2}}{\sigma_{L}}\right)^{0.16}\left(\frac{g \rho_{L}^{2} d_{B}^{3}}{\mu_{L}^{2}}\right)^{0.04}\left(\frac{u_{G}}{\left(g d_{B}\right)^{0.5}}\right)^{0.7}\left\{1+27\left(\frac{u_{G}}{\left(g d_{B}\right)^{0.5}}\right)^{0.52}\left(\frac{\rho_{G}}{\rho_{L}}\right)\right.
$$

where, $b_{1}$ : sparger dependent constant $d_{\mathrm{B}}$ : bubble diameter, $\mathrm{m}$ 
Table 2: Range of column dimensions, physical properties, operating pressures and type of spargers included in the collected databank

\begin{tabular}{|l|l|}
\hline Column Diameter & $0.045-5.5 \mathrm{~m}$ \\
Liquid Density & $681-2965 \mathrm{~kg} \cdot \mathrm{m}^{-3}$ \\
Liquid Viscosity & $0.41-2.95 \mathrm{cP}$ \\
Surface Tension & $20-72 \mathrm{mN} \cdot \mathrm{m}^{-1}$ \\
Gas Density & $0.083-1.2 \mathrm{~kg} \cdot \mathrm{m}^{-3}$ \\
Pressure & $0.1-2 \mathrm{MPa}$ \\
Superficial Gas Velocity & $0.005-0.75 \mathrm{~m} / \mathrm{s}$ \\
Superficial Liquid Velocity & 0 (batch liquid) \\
Overall gas holdup & $0.05-0.685$ \\
\hline
\end{tabular}

Gases: air, $\mathrm{N}_{2}, \mathrm{CO}_{2}, \mathrm{He}, \mathrm{Ar}$, mixture of $\mathrm{N}_{2}$ and $\mathrm{H}_{2}$

Liquids: water, tetradecane, paraffin oil (A, B), soltrol-130, isopropanol, monoethylene glycol, n-heptane, isopar-G etc.

Sparger types: perforated plates with different no. of holes, geometry and hole sizes, single nozzle sparger, cross-sparger, sintered plate etc.

Number of Sources: $60(1965-2000)$

Number of data points: 3500 
Table 3: Set of equations and fitting parameters for the neural network correlation $(I=4$, $\mathrm{J}=10$ )
$E_{O} / M_{O}=\frac{\rho_{L}^{2} d^{2} \sigma_{L}^{2}}{\mu_{L}^{4}}$
$F r_{g}=\frac{u_{g}^{2}}{g d}$
$\operatorname{Re}_{g}=\frac{d u_{g}\left(\rho_{L}-\rho_{g}\right)}{\mu_{L}}$
$D_{R}=\frac{\rho_{g}}{\rho_{L}}$

\begin{tabular}{|l|l|}
\hline $\begin{array}{l}\text { Dimensional } \\
\text { Group }\end{array}$ & Range \\
\hline $\mathrm{E}_{\mathrm{O}} / \mathrm{M}_{\mathrm{O}}$ & $7.5 \mathrm{E} 5-1.14 \mathrm{E} 16$ \\
\hline $\mathrm{Re}_{\mathrm{g}}$ & $5.7-7.1 \mathrm{E} 5$ \\
\hline $\mathrm{Fr}_{\mathrm{g}}$ & $2.3 \mathrm{E}-7-7.35 \mathrm{E}-1$ \\
\hline$\rho_{\mathrm{g}} / \rho_{\mathrm{L}}$ & $8.35 \mathrm{E}-5-2.40 \mathrm{E}-2$ \\
\hline
\end{tabular}

$$
\begin{aligned}
& S_{1}=\frac{\log \left(\varepsilon_{G} / 0.002\right)}{2.64} \\
& U_{1}=\frac{\log \left(\left(E_{o} / M_{o}\right) / 1.97 E 7\right)}{10.2} \\
& U_{2}=\frac{\left.\log \left(F r_{g}\right) / 2.3 E-7\right)}{6.5} \\
& U_{3}=\frac{\log \left(\operatorname{Re}_{g} / 5.71\right)}{5.1} \\
& U_{4}=\frac{\log \left(\left(\rho_{g} / \rho_{L}\right) / 8.3 E-5\right)}{2.46}
\end{aligned}
$$

\begin{tabular}{|c|c|c|c|c|c|c|c|c|c|}
\hline 1 & 2 & 3 & 4 & 5 & 6 & & & & \\
\hline$E+01$ & $9.81 \mathrm{E}+00$ & $-5.78 \mathrm{E}+01$ & $\mathrm{E}+02$ & $1.46 \mathrm{E}+01$ & $-4.25 E+01$ & $-2.00 E+00$ & $-2.64 E+01$ & $7 E+01$ & \\
\hline & & & & & & & & & \\
\hline & & & & & & & & & \\
\hline & -6.8 & & & & & & & & \\
\hline $5.01 \mathrm{E}+00$ & $-1.65 E+01$ & $6.48 \mathrm{E}+01$ & $1.33 E+01$ & $1.46 \mathrm{E}+01$ & $-2.07 E+01$ & $1.82 E+00$ & $-1.62 E+00$ & $-7.11 E+01$ & $-4.72 E+C$ \\
\hline
\end{tabular}

Wij

Wjk

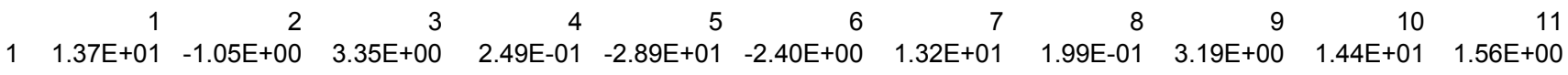

where, $\mathrm{U}_{5}=1, \mathrm{H}_{11}=1$

$H_{j}=\frac{1}{1+\exp \left[-\sum_{i=1}^{I+1} w_{i j} U_{i}\right]}$

$S_{1}=\frac{1}{1+\exp \left[-\sum_{j=1}^{J+1} w_{j k} H_{j}\right]}=\frac{\log \left(\varepsilon_{G} / 0.002\right)}{2.64}$

Overall Gas holdup $\left(\varepsilon_{G}\right)$ 
Table 4: Comparison of ANN and previous literature correlations

\begin{tabular}{|l|c|c|}
\hline Correlation & $\begin{array}{c}\text { AARE } \\
(\mathbf{\%})\end{array}$ & $\begin{array}{c}\text { Standard Deviation } \\
\mathbf{( \% )}\end{array}$ \\
\hline Akita and Yoshida (1973) & 27 & 32 \\
\hline Hikita et al. $(1981)$ & 25 & 20 \\
\hline Hammer et al. $(1984)$ & 37 & 26 \\
\hline Idogawa et al. $(1985)$ & 24 & 24 \\
\hline Reilly et al. $(1986)$ & 28 & 47 \\
\hline Dharwadkar et al. $(1987)$ & 47 & 45 \\
\hline Idogawa et al. $(1987)$ & 54 & 10 \\
\hline Wilkinson et al. $(1992)$ & 25 & 20 \\
\hline Krishna et al. $(1996)$ & 29 & 23 \\
\hline Kojima et al. $(1997)$ & 48 & 49 \\
\hline Joshi et al. $(1998)$ & 30 & 24 \\
\hline Luo et al. $(1999)$ & 25 & 25 \\
\hline Jordan et al. (2001) & 24 & 19 \\
\hline ANN (This Work) & $\mathbf{1 5}$ & $\mathbf{1 4}$ \\
\hline
\end{tabular}


Table 5: Error analysis for some of the input groups at various $\mathrm{J}$ values

\begin{tabular}{|l|l|l|l|}
\hline \multicolumn{1}{|c|}{ Input Group } & $\mathrm{J}=10$ & $\mathrm{~J}=13$ & $\mathrm{~J}=16$ \\
\hline$(\mathrm{Eo} / \mathrm{Mo}), \mathrm{Re}_{\mathrm{g}}, \mathrm{Fr}_{\mathrm{g}}, \mathrm{Ga}$ & $\mathrm{AARE}=21$ & $\mathrm{AARE}=18$ & $\mathrm{AARE}=15$ \\
& $\sigma=26$ & $\sigma=23$ & $\sigma=18$ \\
\hline$(\mathrm{Eo} / \mathrm{Mo}), \mathrm{Re}_{\mathrm{g}}, \mathrm{Fr}_{\mathrm{g}}, \mathrm{D}_{\mathrm{R}}, \mathrm{Ga}$ & $\mathrm{AARE}=22$ & $\mathrm{AARE}=19$ & $\mathrm{AARE}=16$ \\
& $\sigma=20$ & $\sigma=18$ & $\sigma=14$ \\
\hline$(\mathrm{Eo} / \mathrm{Mo}), \mathrm{Re}_{\mathrm{g}}, \mathrm{Fr}_{\mathrm{g}}, \mathrm{We}_{\mathrm{g}}$ & $\mathrm{AARE}=17$ & AARE $=18$ & AARE $=18$ \\
& $\sigma=20$ & $\sigma=18$ & $\sigma=15$ \\
\hline$(\mathrm{Eo} / \mathrm{Mo}), \mathrm{Re}_{\mathrm{g}}, \mathrm{Fr}_{\mathrm{g}}, \mathrm{D}$ & AARE $=28$ & AARE $=22$ & AARE $=19$ \\
& $\sigma=15$ & $\sigma=17$ & $\sigma=18$ \\
\hline$(\mathrm{Eo} / \mathrm{Mo}), \mathrm{Re}_{\mathrm{g}}, \mathrm{Fr}_{\mathrm{g}}, \mathrm{D}_{\mathrm{R}}, \mathrm{Ga}, \mathrm{We}_{\mathrm{g}}$ & AARE $=20$ & AARE $=13$ & AARE $=14$ \\
& $\sigma=15$ & $\sigma=15$ & $\sigma=14$ \\
\hline $\mathbf{( E o / M o}), \mathbf{R e}_{\mathbf{g}}, \mathbf{F r}_{\mathrm{g}}, \mathbf{D}_{\mathbf{R}}$ & $\mathbf{A A R E}=\mathbf{1 5}$ & AARE $=17$ & AARE $=16$ \\
& $\sigma=\mathbf{1 4}$ & $\sigma=15$ & $\sigma=13$ \\
\hline
\end{tabular}

$$
\begin{aligned}
& G a=\frac{\rho_{L}^{2} g d^{3}}{\mu_{L}^{2}} \\
& W e_{G}=\frac{\rho_{G} u_{G}^{2} d}{\sigma_{L}} \\
& D=\frac{u_{G}^{2} \rho_{G}}{g \sigma_{L}}
\end{aligned}
$$


Table 6: Statistical analysis for different liquids using ANN correlation

\begin{tabular}{|l|l|l|l|l|l|l|}
\hline Liquid & $\begin{array}{l}\text { Density } \\
\left(\mathrm{kg} / \mathrm{m}^{3}\right)\end{array}$ & $\begin{array}{l}\text { Viscosity } \\
(\mathrm{CPs})\end{array}$ & $\begin{array}{l}\text { Surface } \\
\text { Tension } \\
(\mathrm{mN} / \mathrm{m})\end{array}$ & $\begin{array}{l}\text { AARE } \\
(\%)\end{array}$ & $\begin{array}{l}\sigma \\
(\%)\end{array}$ & $\begin{array}{l}\text { No. } \\
\text { of } \\
\text { data } \\
\text { points }\end{array}$ \\
\hline water & 998 & 1 & 72 & 16 & 13 & 1780 \\
\hline n-heptane & 681 & 0.41 & 20 & 15 & 13 & 75 \\
\hline tetradecane & 763 & 2.2 & 27 & 14 & 13 & 165 \\
\hline Tetrabromoethane & 2965 & 1.17 & 48 & 15 & 16 & 50 \\
\hline
\end{tabular}


Table 7: Statistical analysis at different pressures using ANN correlation

\begin{tabular}{|c|c|c|c|}
\hline Pressure (atm) & AARE (\%) & $\sigma(\%)$ & No. of data points \\
\hline 1 & 16 & 13 & 1730 \\
\hline 5 & 17 & 7 & 122 \\
\hline 10 & 12 & 8 & 41 \\
\hline 15 & 16 & 17 & 35 \\
\hline 20 & 14 & 17 & 25 \\
\hline
\end{tabular}


Table 8: Statistical analysis for different column diameters using ANN correlation

\begin{tabular}{|l|c|l|}
\hline $\begin{array}{c}\text { Diameter } \\
(\mathrm{m})\end{array}$ & $\begin{array}{c}\text { AARE } \\
(\%)\end{array}$ & $\begin{array}{l}\sigma \\
(\%)\end{array}$ \\
\hline 0.045 & 15 & 12 \\
\hline 0.1 & 13 & 13 \\
\hline 0.15 & 13 & 9 \\
\hline 0.3 & 15 & 12 \\
\hline 0.61 & 16 & 5 \\
\hline
\end{tabular}




\section{Figures Captions:}

Fig. 1 Comparison of Kemoun et al. (2001) CT data at atmospheric pressure with various correlations for air-water system

Fig. 2 Comparison of Kemoun et al. (2001) CT data at $0.7 \mathrm{MPa}$ with various literature correlations for air-water system

Fig. 3 Architecture of the three-layered feedforward neural network with a single hidden layer

Fig. 4 Parity plot for ANN correlation using the whole databank (AARE $=15 \%)$

Fig. 5 Parity plot for ANN and selected literature correlations

Fig. 6 Parity plot for ANN correlation using gas-water system $($ AARE $=16 \%)$

Fig. 7 Effect of column diameter on the overall gas holdup for air-water system at atmospheric pressure using ANN correlation

Fig. 8 Effect of operating pressure for air-water system in $0.15 \mathrm{~m}$ column using ANN correlation

Fig. 9 Comparison of ANN Prediction with experimental data of Reilly et al. (1994) and Vermeer et al. (1981) 


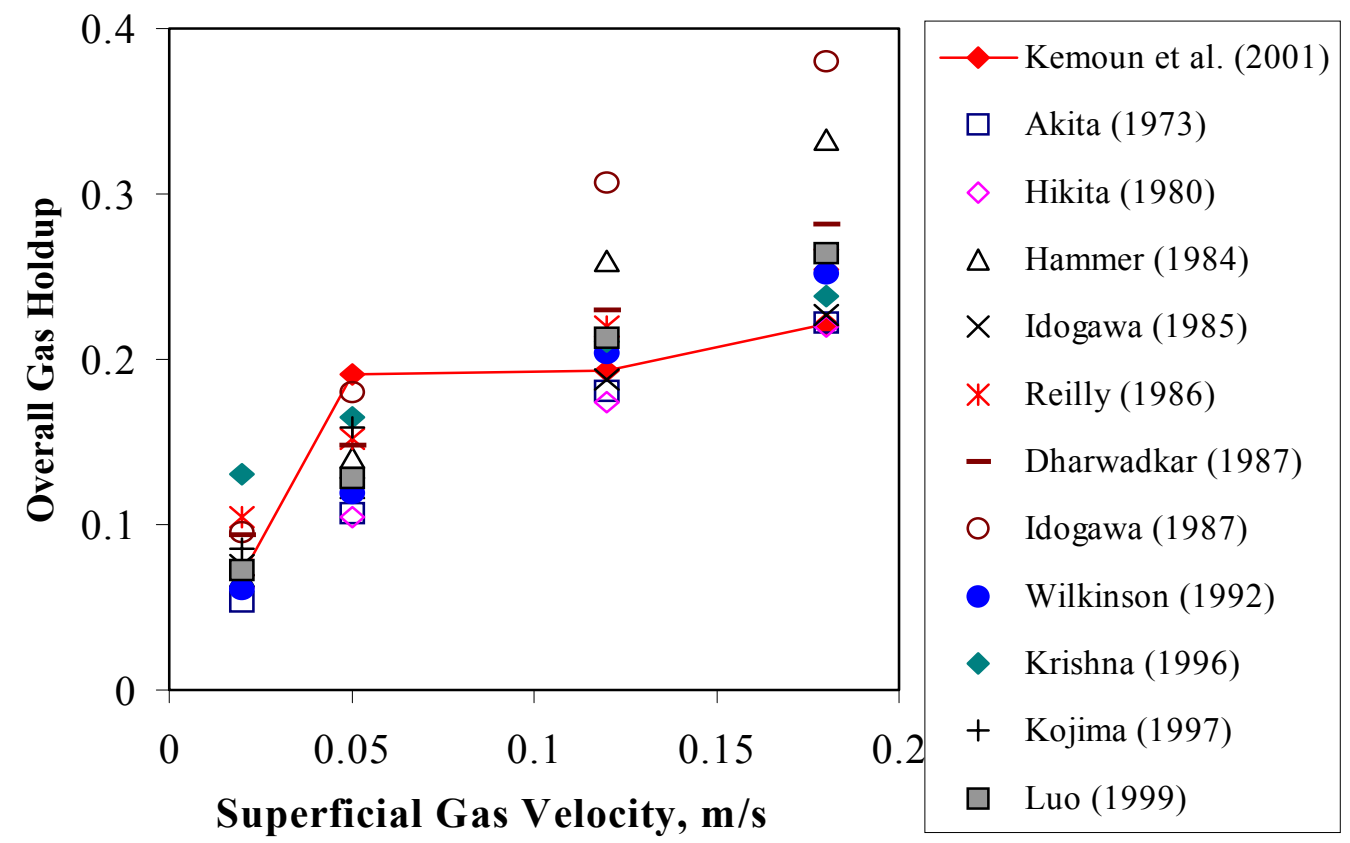

Figure 1: Comparison of Kemoun et al. (2001) CT data at atmospheric pressure with various correlations for air-water system 


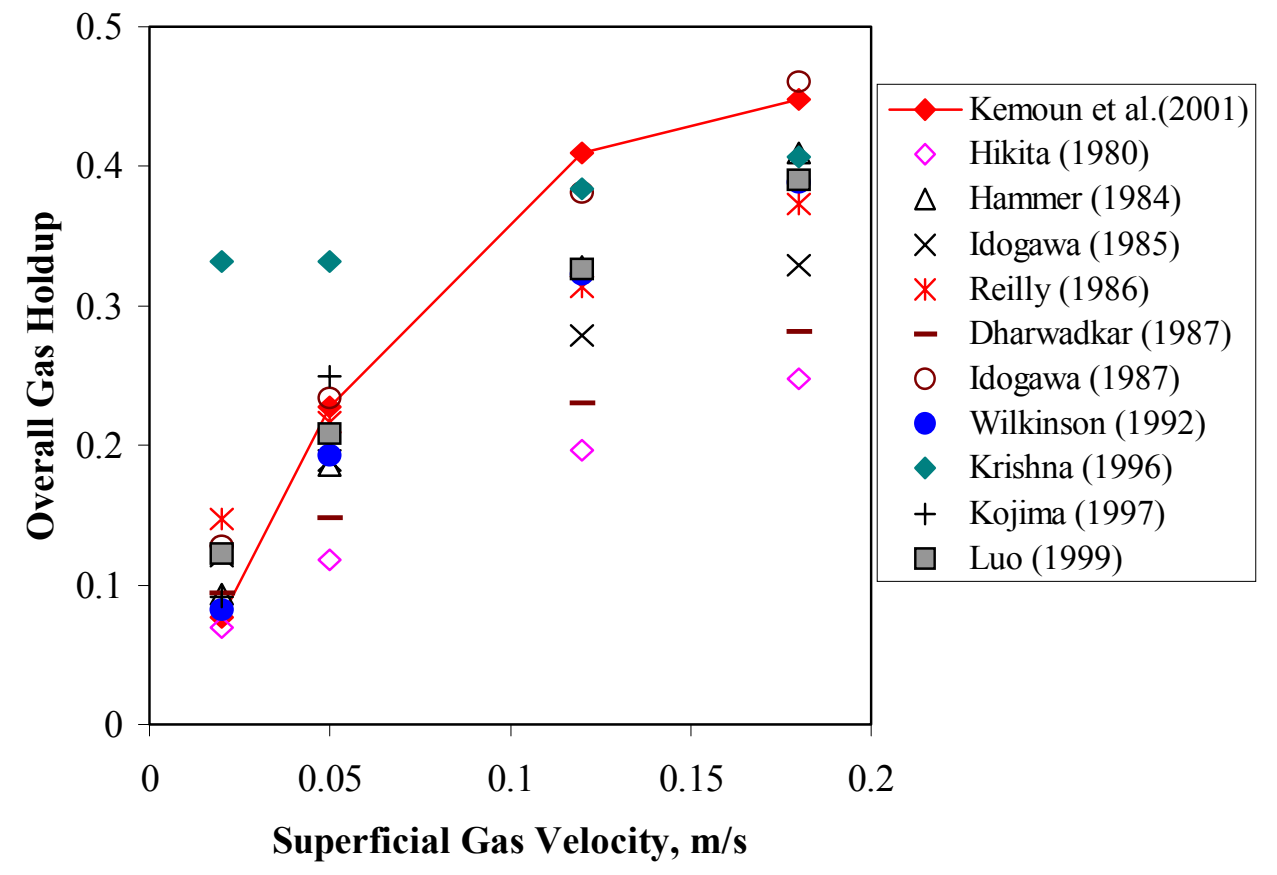

Figure 2: Comparison of Kemoun et al. (2001) CT data at $0.7 \mathrm{MPa}$ with various literature correlations for air-water system 


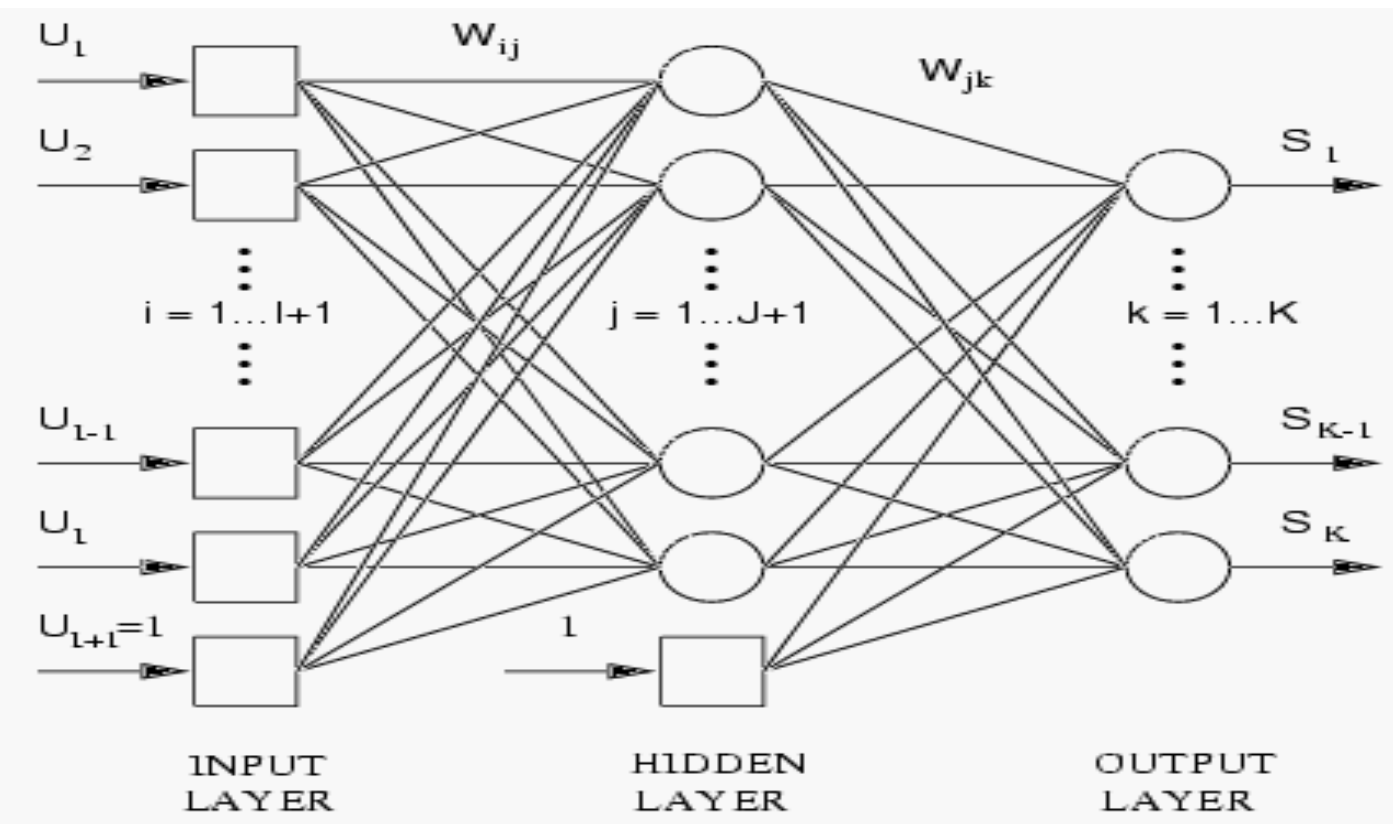

Figure 3: Architecture of the three-layered feedforward neural network with a single hidden layer 


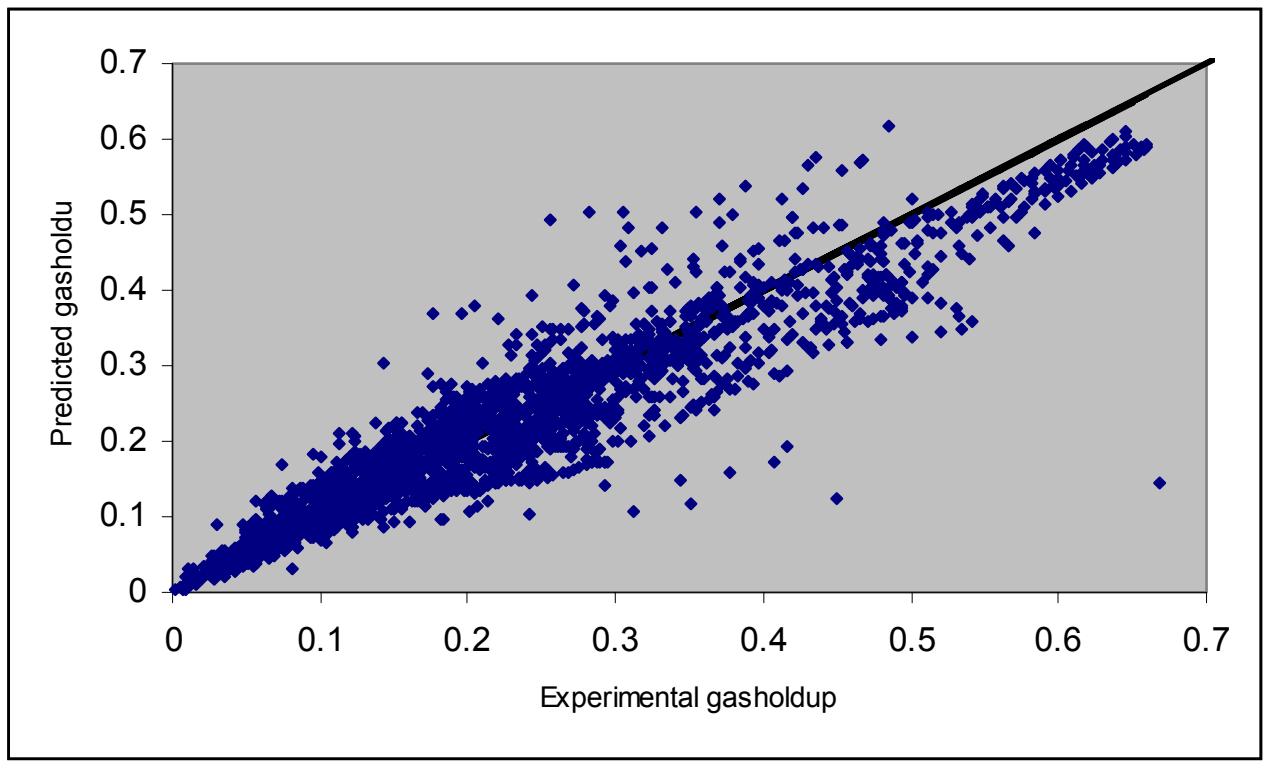

Figure 4: Parity plot for ANN correlation using the whole databank (AARE $=15 \%$ ) 


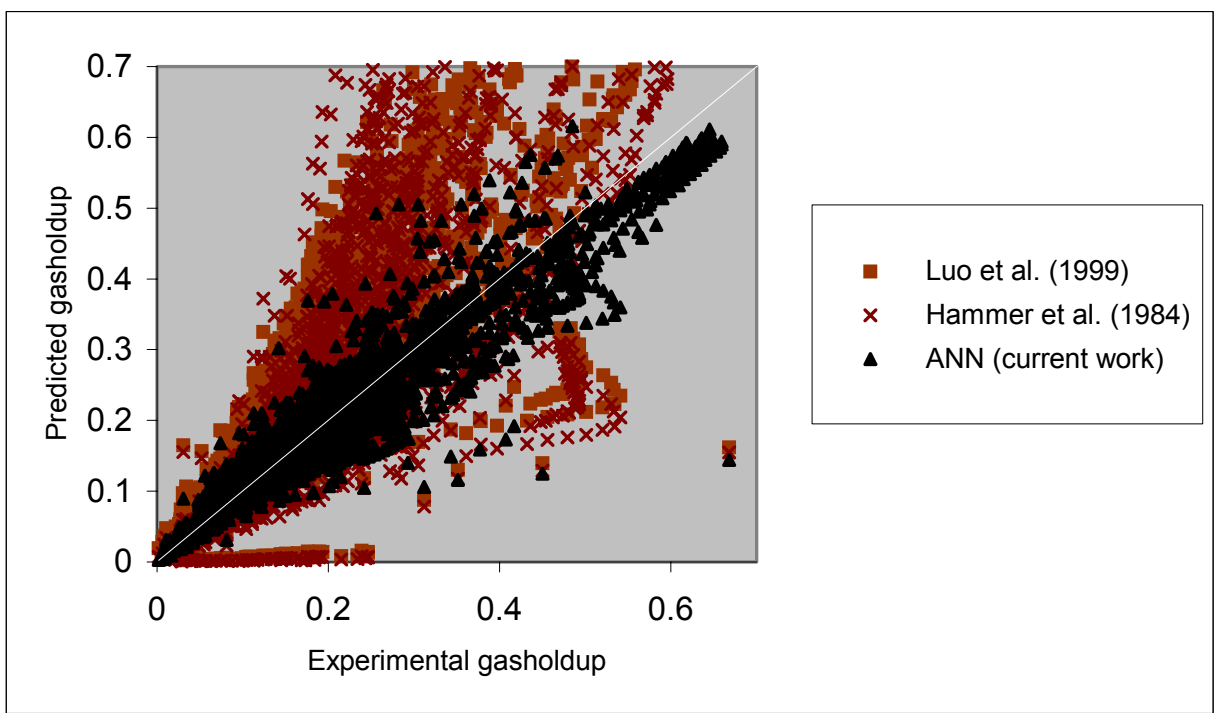

Figure 5: Parity plot for ANN and selected literature correlations 


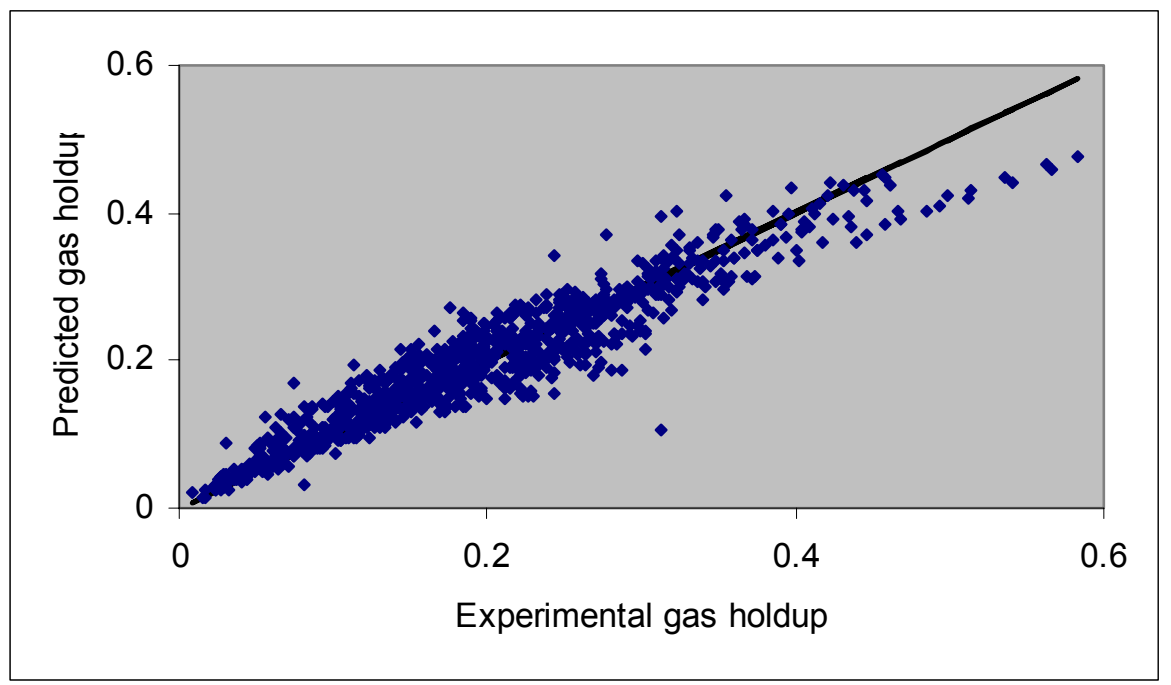

Figure 6: Parity plot for ANN correlation using gas-water system $(\mathrm{AARE}=16 \%)$ 


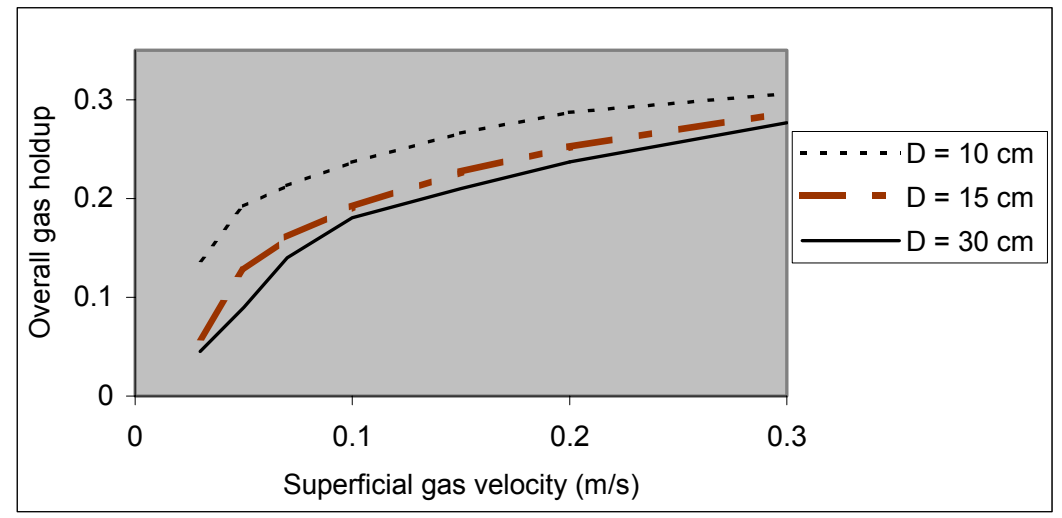

Figure 7: Effect of column diameter on the overall gas holdup for air-water system at atmospheric pressure using ANN correlation 


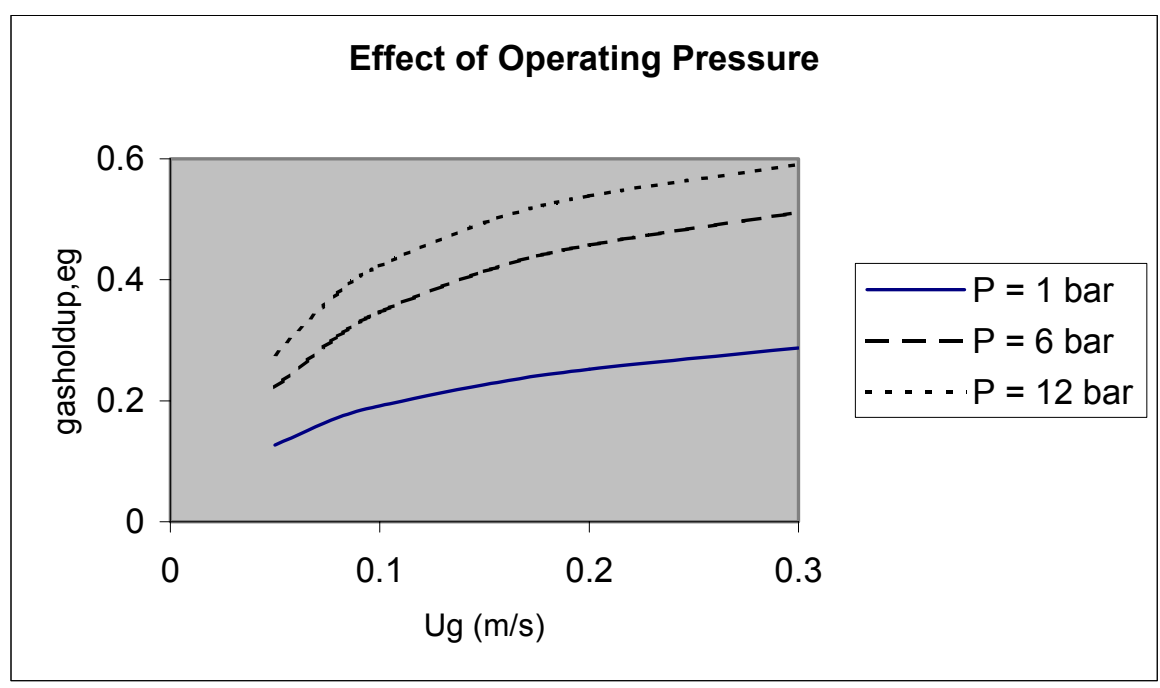

Figure 8: Effect of operating pressure for air-water system in $0.15 \mathrm{~m}$ column using ANN correlation 


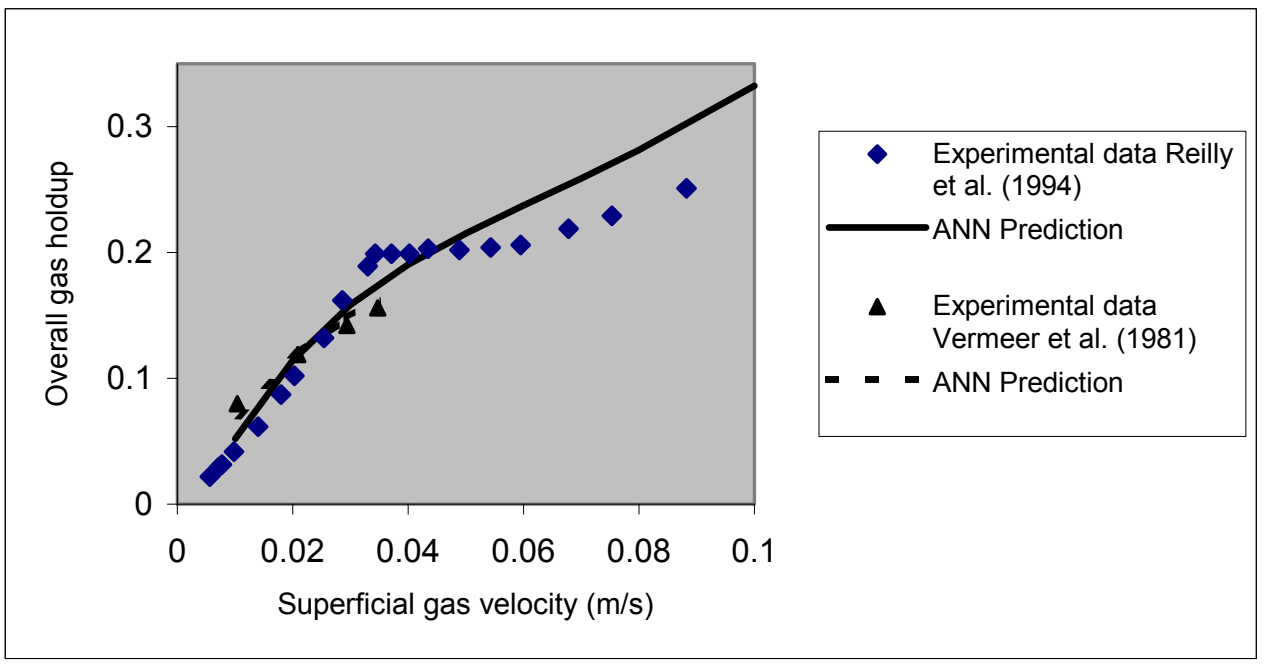

Figure 9: Comparison of ANN Prediction with experimental data of Reilly et al. (1994) and Vermeer et al. (1981) 


\section{APPENDIX D}

\section{PREDICTION OF MASS TRANSFER COEFFICIENT IN BUBBLE COLUMNS OPERATED AT HIGH PRESSURE BASED ON ATMOSPHERIC PRESSURE DATA}




\section{PREDICTION OF MASS TRANSFER COEFFICIENT IN BUBBLE COLUMNS OPERATED AT HIGH PRESSURE BASED ON ATMOSPHERIC PRESSURE DATA}

The liquid volumetric mass transfer coefficient is considered an important design parameter for bubble columns. Consequently, many authors have experimentally determined the values of mass transfer coefficient and developed empirical equations for their estimation (Akita and Yoshida, 1973; Hikita et al., 1981; Hammer et al., 1984; Ozturk et al., 1987). However, these published empirical equations do not account for the effect of pressure, in spite of the fact that the increase in gas hold up and decrease in bubble size with increased pressure leads to a higher interfacial area and mass transfer coefficient. Therefore, the mass transfer coefficient in a high-pressure bubble column will be underestimated by the published empirical correlations. Thus, an accurate estimation of the volumetric mass transfer coefficient for high pressure conditions requires experiments at high pressure, which are more complicated than those at atmospheric pressure.

Very few studies of the mass transfer coefficient at high pressure condition have been reported in the literature. Letzel et al., (1999) measured the mass transfer coefficient in bubble column reactors at elevated pressure by using the dynamic oxygen desorption method. They found that the ratio of volumetric mass transfer coefficient to gas holdup $\left(\mathrm{k}_{\mathrm{L}} \mathrm{a} / \varepsilon_{g}\right)$ is constant and equal to approximately one half up to system pressure of 1.0 $\mathrm{MPa}$. However when gas hold up is larger than $35 \%$, the scatter in $\mathrm{k}_{\mathrm{L}}$ a increases due to the problems with the probe. Kojima et al., (1997) measured the volumetric mass transfer coefficient in bubble columns under pressurized conditions with different liquid phases and with different diameters of the single nozzle used as gas disperser. An empirical correlation was obtained for volumetric mass transfer by considering the effect of pressure and diameter of single nozzle with four empirical constants as fitted parameters, in addition, gas hold up correlation is needed to calculate the mass transfer coefficient. Dewes and Schumpe (1997) reported very strong effects of gas density on gas-liquid mass transfer and the gas density effect increased with the gas velocity. The pressure range in their study was similar to that used by Letzel (1999) and Kojima (1997).

The objective of this study is to develop a procedure for prediction of the volumetric mass transfer coefficient at any pressure based on atmospheric pressure data.

\section{Procedure development}

Wilkinson (1991) recommended accounting for the pressure effect by using the following equation:

$$
\frac{\left(k_{L} a\right)_{P}}{\left(k_{L} a\right)_{a}}=\left[\frac{\left(\varepsilon_{G}\right)_{P}}{\left(\varepsilon_{G}\right)_{a}}\right]^{M}
$$

where subscript $\mathrm{P}$ means pressure conditions and a indicates atmospheric conditions. This allow one to calculate $\mathrm{k}_{\mathrm{L}} \mathrm{a}$ in pressurized bubble columns from atmospheric data for $\mathrm{k}_{\mathrm{L}} \mathrm{a}$ and gas hold up, provided the gas hold up at elevated pressure is also known. However, 
due to the complex hold up structure, $\mathrm{M}$ depends on physical properties and flow regime (Deckwer et al.,1993). Therefore, the approach suggested by Wilkinson is of limited applicability (Grund et al., 1992). To improve the procedure recommended by Wilkinson, a correlation for $\mathrm{M}$ was developed by considering physical properties, column dimension and operation conditions. The following approach was used :

a. Chose proper correlations for the quantities in equation (1).

At atmospheric conditions, Akita and Yoshida's correlation is chosen for $\mathrm{k}_{\mathrm{L}} \mathrm{a}$ calculation since it has been proven to be applicable for scale up (Deckwer et al.,1993)

$\frac{k_{L} a D^{2}}{D_{L}}=0.6\left(\frac{\mu_{L}}{\rho_{L} D_{L}}\right)^{0.5}\left(\frac{g \rho_{L} D^{2}}{\sigma}\right)^{0.62}\left(\frac{g \rho_{L}^{2} D^{3}}{\mu_{L}^{2}}\right)^{0.31} \varepsilon_{G}^{1.1}$

To predict gas holdup the correlation of Luo et al.(1999) was used at both low pressure and high pressure since it can cover a wide range of operating conditions and systems of different physical properties,

$$
\frac{\varepsilon_{G}}{1-\varepsilon_{G}}=2.9 \frac{\left(\frac{U_{G}^{4} \rho_{G}}{\sigma g}\right)^{\alpha}\left(\frac{\rho_{G}}{\rho_{L}}\right)^{\beta}}{\left[\cosh \left(M o_{L}\right)^{0.054}\right]^{4.1}}
$$

Where $\mathrm{Mo}_{\mathrm{L}}=g \mu_{L}^{4} /\left(\rho_{L}-\rho_{G}\right) \sigma^{3}, \alpha=0.21 M o_{L}^{0.0079}$ and $\beta=0.096 M o_{L}^{-0.011}$

Substituting equations (2) for $\left(\mathrm{k}_{\mathrm{L}} \mathrm{a}\right)_{\mathrm{a}}$ to equation (1), and assuming $\left(\varepsilon_{G}^{1.1} / \varepsilon_{G}^{M}\right)_{\text {atm }}=1$ to simplify the problem, one can obtains the following equation:

$$
\frac{k_{L} a D^{2}}{D_{L}}=0.6 S c^{0.5} E o^{0.62} G a^{0.31}\left(\varepsilon_{G}\right)_{P}^{M}
$$

where $\left(\varepsilon_{G}\right)_{P}$ can be evaluated from equation (3).

b. Develop a correlation for $\mathrm{M}$

In equation (4) parameter $M$ depends on physical properties and flow regime which is associated with the operating conditions and column dimensions as mentioned above. To account for these factors, a correlation was developed by Wu et al.(1999):

$$
n=2.188 \times 10^{3} \operatorname{Re}^{-0.598} \mathrm{Fr}^{0.146} M_{o L}^{-0.004}
$$

for prediction of the exponent $\mathrm{n}$ in the gas radial gas hold up profile which is usually represented by 
$\varepsilon_{G}=\widetilde{\varepsilon}_{G}\left(\frac{n+2}{n}\right)\left[1-c(r / R)^{n}\right]$

$\mathrm{n}$ indicates the steepness of hold up profile and reflect the intensity of liquid circulation. It is depends on flow characteristics and nature of system used as well. $\mathrm{n}$ and $\mathrm{M}$ must be somehow related. Then $M=f(n)$ can be obtained by fitting part of the experimental data reported in the literature using equation (1). We have obtained 155 sets of experimental data available from the literature and chosen $65 \%$ of the points to obtain the $\mathrm{M}$ dependence on $\mathrm{n}$ as follows:

$M=0.3 \ln (n)+0.044$

Now one can predict the mass transfer coefficient based on gas hold up data only by using equations (3)-(6). We have compared the model predictions with experimental data at the range of pressure $0.1 \mathrm{MPa}-1.1 \mathrm{MPa}$. Some of results are shown below.

\section{Comparison of model prediction and experimental data}

At elevated pressure, experimental data has been reported by Letzel (1999) at 0.1MPa to 1.0 MPa system pressure with column diameter equal to $0.15 \mathrm{~m}$ using dynamic oxygen desorption method. The comparison of model prediction and the reported experimental data by Letzel (1999) at 0.2MPa system pressure is shown in Figure 1. From Figure 1 one can see that the model predicts the experimental data well. The prediction by the correlation of Akita (1973) under-estimates the experimental data even if using hold up data at $0.2 \mathrm{MPa}$. This correlation usually provides for a conservative estimate as reported by Deckwer et al.(1993). For the pressure at 0.3, 0.4 MPa or higher, the comparison of model prediction and experimental data is similar to what is discussed above and mass transfer coefficient increases with increasing system pressure due to small bubble size and an increase in the number of small bubbles which results in higher gas hold up. In addition, parameter $\mathrm{M}$ in equation (1) was reported by Wilkinson(1992, 1994) to be equal to 1-1.2. If $\mathrm{M}$ is set equal to 1.1 , one can apply equation (2) and (3) in equation (1), then the correlation of Akita returns the same formula except that the hold up needs to be calculated by the correlation obtained at elevated pressure condition. From this point of view, one can argue that the procedure suggested by Wilkinson does not predict the experimental data well without considering the dependence of $\mathrm{M}$ on physical properties and flow regime. When the dependence of $\mathrm{M}$ on physical properties and flow regime, as suggested by Deckwer et al.(1993), is accounted for the prediction for $\mathrm{k}_{\mathrm{L}}$ a is good. In this study $\mathrm{M}$ was found to vary from 0.4 to 1.1 depending on system pressure and superficial gas velocity.

The other sets of experimental data for volumetric mass transfer coefficient at high pressure was obtained by Kojima (1997) using oxygen electrode (Oxi-96WTW) to measure dissolved oxygen. The column diameter used was small $(0.045 \mathrm{~m})$ and the system pressure range employed was 0.1-1.1 MPa. The comparison of model prediction and experimental data at $0.6 \mathrm{MPa}$ is shown in Figure 2. From Figure 2, it is clear that the model proposed in this work can predict experimental observation well. Again either 
Akita's correlation or equation (1) with $\mathrm{M}=1.1$ predicts a lower mass transfer coefficient than experimental data.

The comparison of additional experimental data and model prediction is shown in Figure 3. From Figure 3, one can see that for most of the available experimental data the error between predicted mass transfer coefficient by this work and experimental data reported in the literature is less than $20 \%$ within the pressure range 0.1 to $1.1 \mathrm{MPa}$. There is another set of experimental results reported by Dewes et al.(1997), The data was not included in Figure 3 due to insufficient information on physical properties to be used in the proposed model. However, Dewes et al.(1997) reported that $k_{L} a \propto \rho_{G}^{0.45-0.5}$ and this is comparable with this work regarding the dependence of mass transfer coefficient on gas density.

\section{SUMMARY}

Based on the approach that mass transfer coefficient and gas hold up data obtained at lower pressure and gas hold up obtained at high pressure conditions can be used to predict the mass transfer coefficient at high pressure, we have chosen the widely accepted mass transfer correlation and newly reported gas hold up correlation which covers wide operating pressure conditions to form a new correlation for the prediction of mass transfer coefficient at wide range of operating conditions. The correlation can be used to predict the mass transfer coefficient up to $1.1 \mathrm{MPa}$ system pressure with error within $20 \%$.

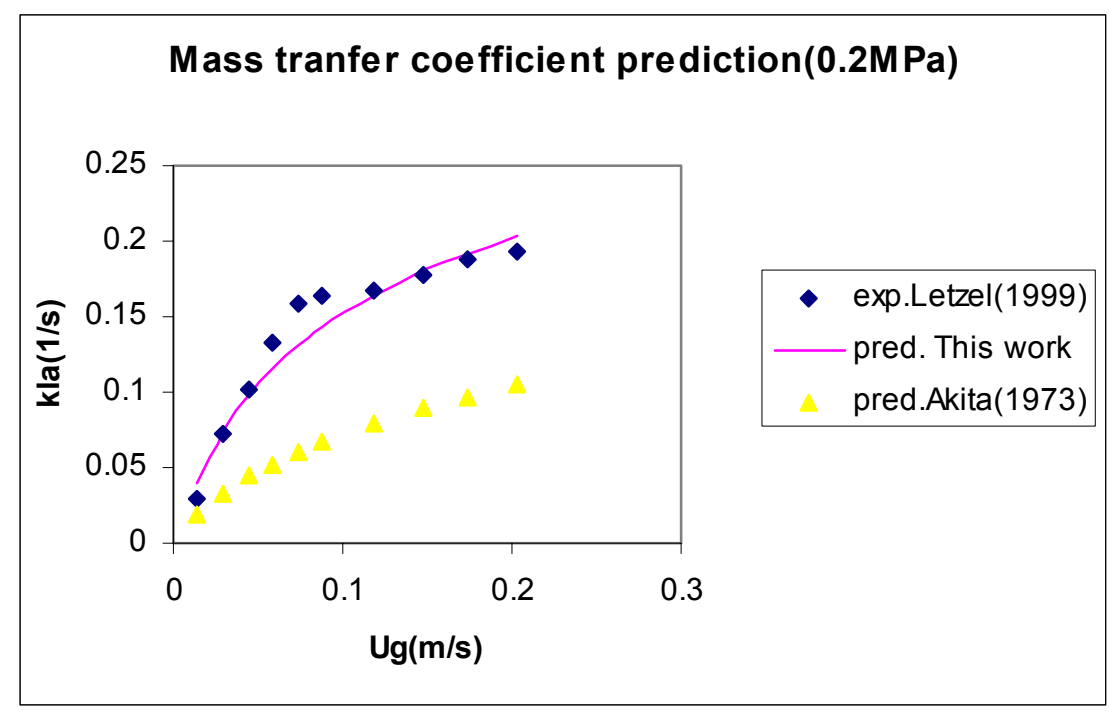

Figure 1 Comparison of model prediction and experimental data of Letzel (1999) 


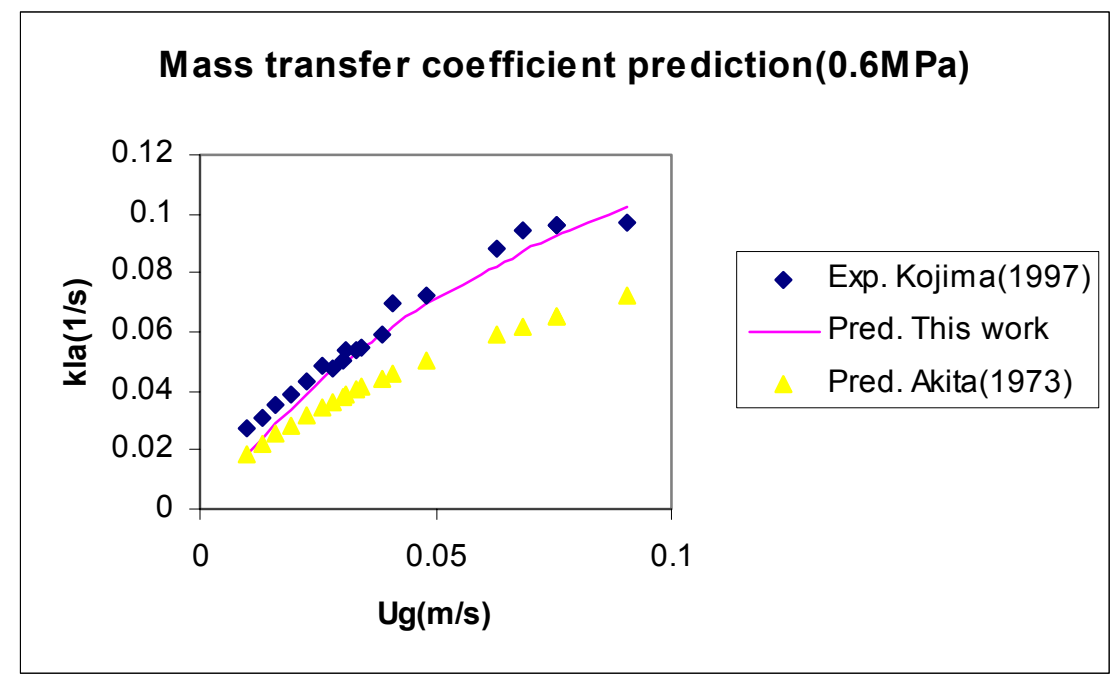

Figure 2 Comparison of model prediction and experimental data by Kojima(1997)

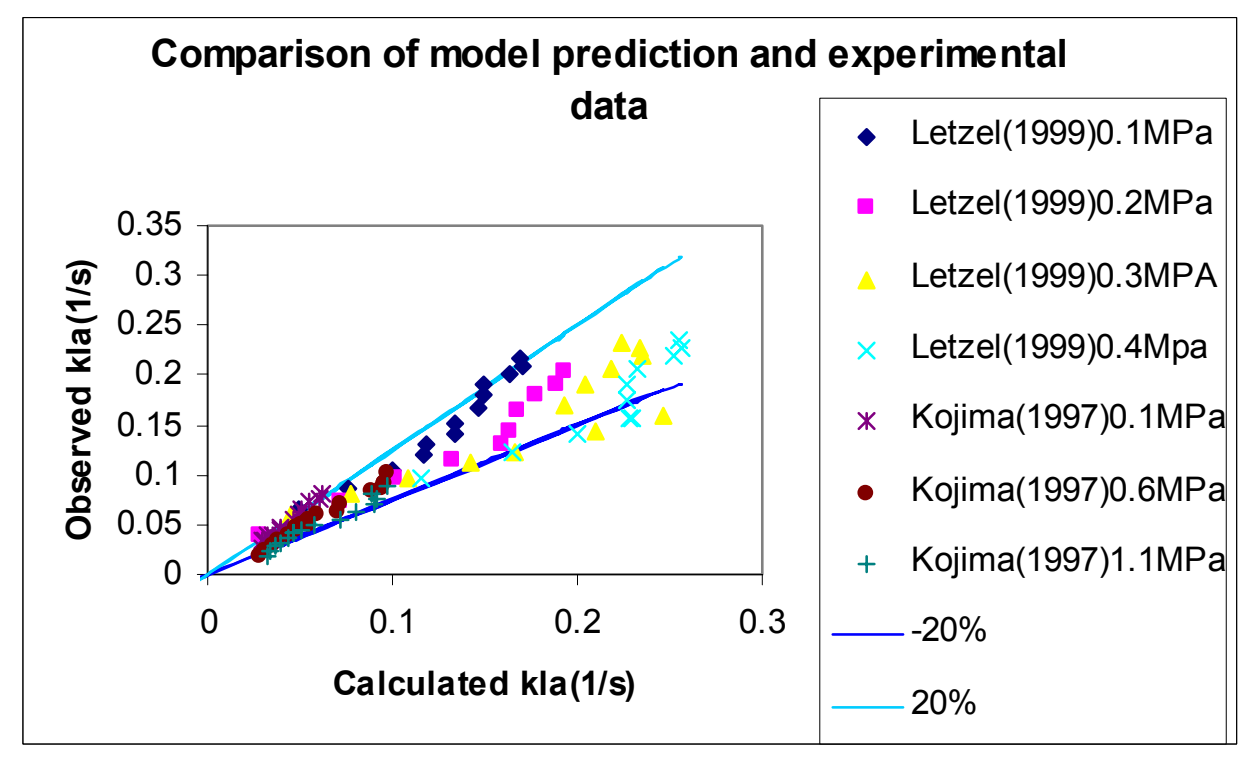

Figure 3 Comparison of predicted $\mathrm{k}_{\mathrm{L}} \mathrm{a}$ and observed $\mathrm{k}_{\mathrm{L}} \mathrm{a}$ 


$\begin{array}{ll}4 & \text { NOMENCLATURE } \\ \mathrm{a} & \text { special interfacial area, } \mathrm{m}^{2} / \mathrm{m}^{3} \\ \mathrm{D} & \text { Column diameter, } \mathrm{m} \\ \mathrm{D}_{\mathrm{L}} & \text { Molecular diffusivity, } \mathrm{m}^{2} / \mathrm{s} \\ \mathrm{Eo} & \text { Eotvos number, dimensionless } \\ \mathrm{Fr}_{\mathrm{g}} & \text { Gas Froude number, dimensionless } \\ \mathrm{g} & \text { Acceleration due to gravity, } \mathrm{m} / \mathrm{s}^{2} \\ \mathrm{Ga} & \text { Galieo number, dimensionless } \\ \mathrm{k}_{\mathrm{L}} \mathrm{a} & \text { Volumetric mass transfer coefficient, } 1 / \mathrm{s} \\ \mathrm{Mo}_{\mathrm{L}} & \text { Liquid Morton number, dimensionless } \\ \mathrm{n} & \text { Parameter in Eq(5) } \\ \mathrm{Re}_{\mathrm{G}} & \text { Reynolds number, dimensionless } \\ \mathrm{Sc}_{\mathrm{Sc}} & \text { Schmidt number, dimensionless } \\ \mathrm{U}_{\mathrm{Sg}} & \text { Superficial gas velocity, m/s }\end{array}$

\section{Greek letters}

$\varepsilon_{G} \quad$ Cross-sectional average gas hold up

$\mu_{L} \quad$ Liquid viscosity, Pa.s

$\rho_{G} \quad$ Gas density, $\mathrm{kg} / \mathrm{m}^{3}$

$\rho_{L} \quad$ Liquid density, $\mathrm{kg} / \mathrm{m}^{3}$

$\sigma_{L} \quad$ Liquid surface tension, $\mathrm{N} / \mathrm{m}$

\section{REFERENCES}

Akita, K.,and F. Yoshida, Gas holdup and volumetric mass transfer coefficient in bubble columns, Ind. Eng. Chem. Proc. Des. Dev., 12, 76, 1973.

Hammer, H, H. Schrag, K. Hektor, K. Schonau, W. Kuster, A. Soemarno, U. Sahabi, and W. Napp, New subfunctions on hydrodynamics, heat and mass transfer for gas/liquid and gas/liquids/solid chemical and biochemical reactors, Front. Chem. Reac. Eng., 464, 1984.

Hikita, H., H. Sasai, K.Tanigawa, K. Segawa, and M. Kitao, The volumetric liquid phase mass transfer coefficient in bubble columns, Chem. Eng. J., 22,61, 1981.

Ozturk, S.S., A. schumpe and W.D. Deckwer, Organic liquids in a bubble column: Hold up and mass transfer coefficients, AICHE J., 33, 1473-1480, 1987.

Letzel, H.M., J.C. Schouten, R.Krishna, C.M. van den Bleek, Gas hold up and mass transfer in bubble column reactors operated at elevated pressure, Chem. Eng. Sci., 54, 2237-2246, 1999.

Kojima H., J. Sawai and H. Suzuki, Effect of pressure on volumetric mass transfer coefficient and gas hold up in bubble columns, Chem. Eng. Sci., 52, 4111-4116, 1997. 
Dewes I. And A. Schumpe, Gas density effect on mass transfer in the slurry bubble column, Chem. Eng. Sci., 52, 4105-4109, 1997.

Deckwer W.-D and A. Schumple, Improved tools for bubble column reactors design and scale up, Chem. Eng. Sci., 48, 889-911, 1993

Grund, G., A. Schumpe and W.D. Deckwer. Gas-liquid mass transfer in a bubble column with organic liquids, Chem. Eng. Sci., 47, 3509-3516, 1992.

Luo X., D.J. Lee, R. Lau, G.Yang and L.S. Fan, Maximum stable bubble size and gas hold up in high pressure slurry bubble columns, AICHE J., 45, 665-680, 1999. 


\section{$\underline{\text { Appendix E }}$}

\section{Phase Distribution in a High Pressure Slurry Bubble Column via Computed Tomography}




\title{
Phase Distribution in a High Pressure Slurry Bubble Column via Computed Tomography
}

\author{
Ashfaq Shaikh, Novica Rados, Muthanna Al-Dahhan \\ Chemical Reaction Engineering Laboratory, Department of Chemical Engineering, Campus Box 1198, 1 \\ Brookings Drive, Washington University St. Louis, MO 63130-4899 USA
}

\begin{abstract}
Due to their numerous advantages, Slurry Bubble Column Reactors (SBCR) are gaining wide importance in petroleum, petrochemical, and chemical industries. Most previous literature studies regarding fluid dynamic parameters in SBCR are limited to low superficial gas velocity and/or atmospheric pressure. Moreover, the presence of a solids phase poses difficulties in probe measurement and 'see through' measurement techniques such as Particle Image Velocimetry (PIV) and Laser Doppler Velocimetry (LDV). Single source $\gamma$-ray Computed Tomography (CT), which is a technique to measure cross-sectional phase holdup distribution in two-phase systems, has been used in the current work. Using a single source $\gamma$-ray CT, a new methodology (CT/Overall gas holdup) has been developed, based on sound assumptions, to calculate phase holdups of all three moving phases. The effects of superficial gas velocity $(8-14 \mathrm{~cm} / \mathrm{s})$ and operating pressure $(0.1-1 \mathrm{MPa})$ on time- and cross-sectionally averaged phase holdups have been investigated in a laboratory scale (diameter $0.162 \mathrm{~m}$ ) SBCR using air-water$(150 \mu \mathrm{m})$ glass beads and air-Therminol LT- $(150 \mu \mathrm{m})$ glass beads systems.
\end{abstract}

Keywords Computed tomography, slurry bubble column, high pressure, gas holdup

\section{INTRODUCTION}

Slurry bubble column reactors (SBCR) are cylindrical vessels in which gas is sparged using a distributor into a suspension of liquid and solid particles. The size of the solid particles ranges from 5 to $150 \mu \mathrm{m}$ and solids loading up to $50 \%$ volume (Krishna, 1997). Gas phase contains one or more reactants while liquid phase usually contains product and/or reactant (or sometimes inert). The solid particles are typically catalyst. These reactors are of considerable interest in processes such as, hydrogenation of heavy oils, Fischer-Tropsch synthesis, liquid phase methanol synthesis. The main advantages of slurry reactors are excellent mixing without moving parts (smaller capital and maintenance costs), and much lower power consumption. Such excellent mixing characteristics lead to good heat and mass transfer and hence, to improved production. One of the main disadvantages of slurry bubble column reactors is significant backmixing, which can affect product conversion. Although slurry bubble column reactors are simple in construction, the proper design and scale-up of such reactors require a thorough understanding of the prevailing hydrodynamic and mixing characteristics associated with it. However, the flow field and fluid dynamics of these reactors are not well understood due to the complex interaction among the three phases.

Most of previous literature work has been focused on gas-liquid systems. There are some studies characterizing hydrodynamics of SBCR but majority of them have been conducted at atmospheric pressure and low superficial gas velocity. Information available at high pressure is mainly limited to overall gas holdup, tracer studies, and pressure drop measurements. Very few studies have been carried out to see the effect of operating parameters on gas holdup profile in slurry bubble column reactors (Warsito, 1997, George, 2001). Also, the solids phase poses difficulty for probe measurement techniques such as 
Pitot tube and optical probe and 'see through' measurement techniques such as Particle Image Velocimetry (PIV) and Laser Doppler Velocimetry (LDV) (Chaouki, 1997).

Single source gamma-ray CT is generally used to evaluate the phase holdup profiles in two phase systems. In three phase systems, such as gas-liquid-solid slurry system, a single source CT may be used only with the help of some physically valid assumptions (Chen, 2001) or with other appropriate holdup measurement technique (Kumar, 1997, George, 2001). Dual source CT or ultrasonic tomography is capable of completely resolving the holdups in three phase systems. However, a dual source gamma-ray CT technique for holdup profiles measurement in slurry bubble columns is not yet available, and the ultrasonic technique is limited to a bubbly flow regime and very low solids holdup because of high nonlinear signal dependency (Warsito, 1995). Recently, using electrical capacitance tomography (ECT), Warsito and Fan (2001) have developed a neural network based algorithm to reconstruct the three phases distribution in an atmospheric slurry bubble column.

The current study investigates the effect of superficial gas velocity and operating pressure on phase holdup profiles in a slurry bubble column reactor by developing a methodology based on a single source $\gamma$-ray CT/Overall gas holdup measurements with sound assumptions to evaluate cross-sectional distribution of three dynamic phases.

\section{EXPERIMENTAL SET UP AND CONDITIONS}

The experiments were carried out in a stainless steel column, which has an inner diameter of $16.15 \mathrm{~cm}$ and a height of $2.5 \mathrm{~m}$. The column is designed to support a maximum operating pressure of 300 psig. The details of experimental set up have been described elsewhere (Kemoun, 2001). Water and Therminol LT were used as liquid phase. The properties of these fluids are shown in Table 1. Glass beads with an average diameter of $150 \mu \mathrm{m}$ and particle density of $2500 \mathrm{~kg} / \mathrm{m}^{3}$ constituted the solids phase. A solids loading of $9.1 \%$ volume was employed. In all performed experiments, the dynamic height of slurry was maintained at $1.8 \mathrm{~m}$ from the distributor, and, therefore, the initial slurry height varied depending on the operating condition. The experiments were carried out at superficial gas velocities of 8 and $14 \mathrm{~cm} / \mathrm{s}$ and operating pressures of 0.1 and $1 \mathrm{MPa}$.

\begin{tabular}{|c|c|c|c|}
\hline Liquid Phase & $\begin{array}{c}\text { Density } \\
\left(\mathrm{kg} / \mathrm{m}^{3}\right)\end{array}$ & $\begin{array}{c}\text { Viscosity } \\
(\mathrm{cP})\end{array}$ & $\begin{array}{c}\text { Surface tension } \\
(\text { dyne/cm })\end{array}$ \\
\hline Water & 998 & 1 & 72 \\
\hline Therminol LT & 886 & 0.88 & 17 \\
\hline
\end{tabular}

Table 1: Physical properties of liquid phases used

\section{COMPUTED TOMOGRAPHY (CT)}

As stated earlier, Computed Tomography (CT) is used for the measurement of the cross-sectional phase holdup distribution in multiphase systems (Figure 2). CT technique has been extensively implemented at the Chemical reaction Engineering Laboratory (CREL), Washington University on various multiphase flow systems. The software and hardware details of the single source $\gamma$-ray CT have been explained elsewhere in detail (Kumar, 1994) and hence, will not be repeated here. After detailed analysis of various algorithms, Kumar (1994) implemented Estimation-Maximization Algorithm (EM Algorithm) for image reconstruction. It is based on maximum likelihood principles and takes into account the stochastic nature of the projection measurements.

While single source CT reconstructs two-phase holdup profiles, the three-phase system under investigation requires one additional source to completely resolve its phase holdup profiles. To evaluate holdup profiles of all three dynamic phases in a slurry system using single $\gamma$-ray source, a new method has been proposed viz; CT/Overall gas holdup method (Rados, 2003), which combines CT and overall 
gas holdup measurements along with sound assumptions based on previous literature findings. The assumptions are

i) gas holdup is axially invariant i.e. cross-sectional gas holdup is equal to overall gas holdup

ii) cross-sectional solids loading is uniform.

Overall gas holdup is obtained by measuring the change in slurry height as follows

$\varepsilon_{G}=\frac{H_{d}-H_{S}}{H_{d}}$

For a single $\gamma$ radiation source, absorbance $A$ over the path $l$ is equal to

$A=-\ln \frac{I}{I_{0}}=\sum_{l}(\rho \mu)_{i j} l_{i j}$

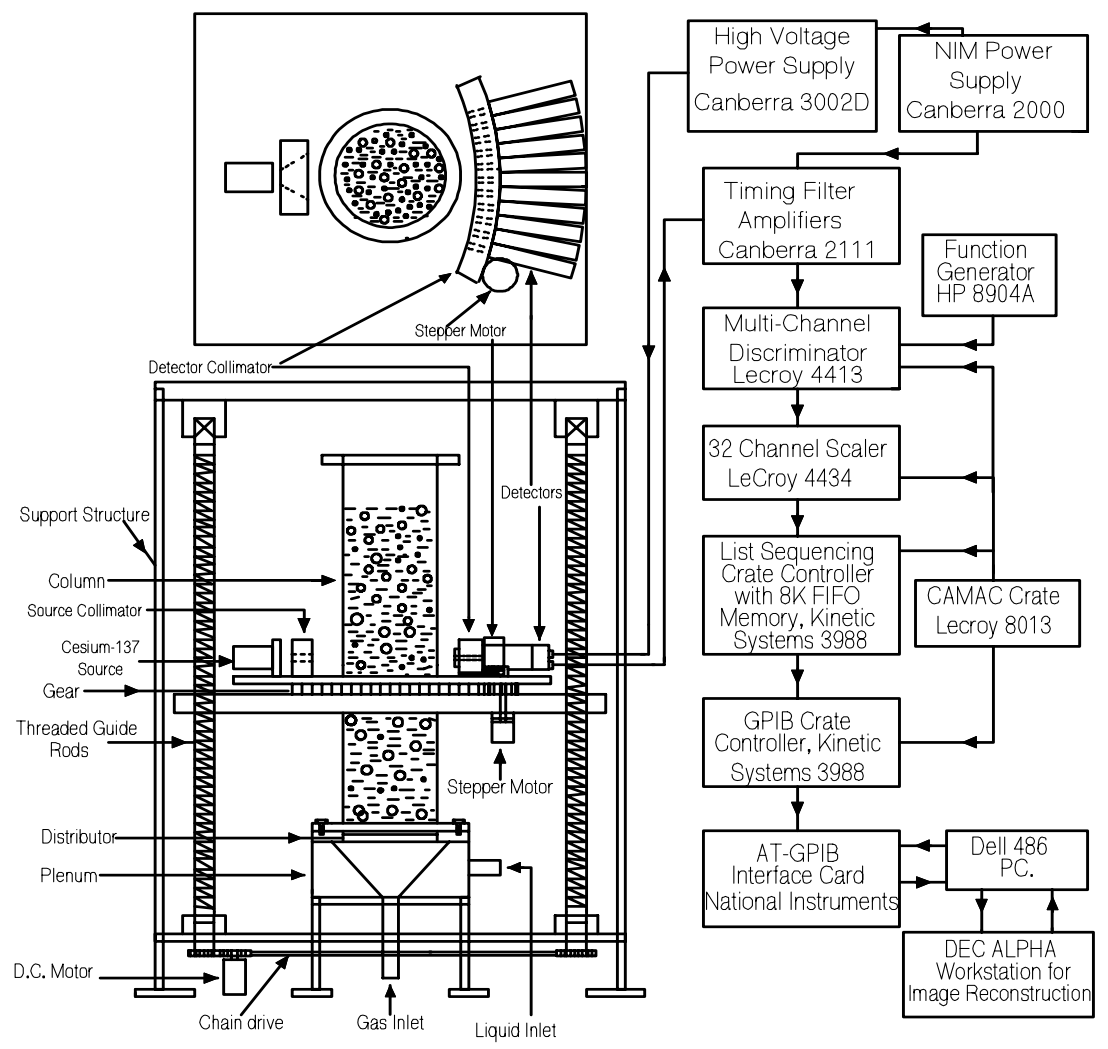

Figure 2: Configuration of the CT experimental setup (Kumar, 1994)

To get the holdup distribution, we have to measure the absorbance $A_{K}$ for an empty column $(K=G)$, for a column filled with liquid $(K=L)$, for a column filled with solids and gas in voids between solid particles $(K=G S)$, and for a column in operation with gas-liquid-solid slurry $(K=G L S)$. For each of these scans, the detected intensity of radiation $I_{K}$, and hence the measured absorbance $A_{K}$, are different. Since the flow is time dependent, a larger number of acquired projections than cells (\#equations >> \#unknowns, over sampling) will yield more accurate time-averaged attenuation coefficients. In general, $I_{0}$ is unknown, and 
because of that the intensity of radiation $I_{K}$ needs to be normalized with the intensity of radiation detected in the column containing only the gas phase $I_{G}$.

$$
A_{K}=-\ln \frac{I_{K}}{I_{G}}=\sum_{l}\left[(\rho \mu)_{K, i j}-(\rho \mu)_{G, i j}\right]_{i j}
$$

Relative volumetric attenuation has been defined as

$$
R_{K, i j}=(\rho \mu)_{K, i j}-(\rho \mu)_{G, i j}
$$

The volumetric attenuation coefficient for a slurry system is

$$
(\rho \mu)_{G L S, i j}=(\rho \mu)_{G, i j} \varepsilon_{G, i j}+(\rho \mu)_{L, i j}\left(1-\varepsilon_{G, i j}-\varepsilon_{S, i j}\right)+(\rho \mu)_{S, i j} \varepsilon_{S, i j}
$$

The volumetric attenuation of solids can be calculated by writing equation for a packed bed of solids (GS) with uniform holdup $\varepsilon_{S}^{0}$ and gas in voids between the solid particles, which with equation (3b) yield

$$
(\rho \mu)_{S, i j}=\frac{R_{G S, i j}+(\rho \mu)_{G, i j} \varepsilon_{S}^{0}}{\varepsilon_{S}^{0}}
$$

The combination of equation (4) with equation (3b) (written for $\mathrm{K}=\mathrm{L}$, and $\mathrm{K}=\mathrm{GLS}$ ) and equation (5) results in the following expression for local gas holdup in each cell ij

$$
\varepsilon_{G, i j}=\frac{R_{G S, i j} \frac{\varepsilon_{S, i j}}{\varepsilon_{S}^{0}}+R_{L, i j}\left(1-\varepsilon_{S, i j}\right)-R_{G L S, i j}}{R_{L, i j}}
$$

In order to close the above equation with two unknowns $\left(\varepsilon_{\mathrm{G}, \mathrm{ij}}\right.$ and $\left.\varepsilon_{\mathrm{S}, \mathrm{ij}}\right)$, we need one more equation. In the current work, the needed equation has been obtained from the pressure drop across the plane of the CT scan and overall gas holdup measurement.

The generalized pressure drop equation for slurry bubble column is

$$
-\frac{1}{\mathrm{~g}} \frac{\Delta \mathrm{P}}{\Delta \mathrm{z}}=\rho_{\mathrm{G}} \overline{\varepsilon_{\mathrm{G}}}+\rho_{\mathrm{L}}\left(1-\overline{\varepsilon_{\mathrm{G}}}-\overline{\varepsilon_{\mathrm{S}}}\right)+\rho_{\mathrm{S}} \overline{\varepsilon_{\mathrm{S}}}
$$

The above equation can be rearranged as

$$
\overline{\varepsilon_{S}}=A+B \overline{\varepsilon_{G}}
$$

A is a function of the pressure drop measurement. Due to the sensitivity of the results to the pressure drop measurement across the CT scan plane, $A$ is considered here as a fitted parameter. $B$ is a function of solids and liquid densities (Rados, 2003).

The solids loading across the cross-section is defined as 


$$
\overline{v_{S}}=\frac{\overline{\varepsilon_{S}}}{1-\overline{\varepsilon_{G}}}
$$

With the assumption (ii) of uniform cross-sectional solids loading, the solids holdup distribution can be calculated based on equation (9) as

$$
\varepsilon_{S, i j}=\overline{v_{S}}\left(1-\varepsilon_{G, i j}\right)
$$

Combining equations (6), (8), (9), and (10) yields the following single source CT equation for gas holdup distribution

$$
\varepsilon_{G, i j}=1-\frac{R_{G L S, i j}}{\overline{\frac{v_{S}}{\varepsilon_{S}^{0}} R_{G S, i j}}+\left(1-\overline{v_{S}}\right) R_{L, i j}}
$$

The iterative procedure used to compute gas and solids holdup profiles where $A$ is fitted parameter is as follows (Rados, 2003)

1) Guess the value of the adjusted parameter $A$ based on the range of pressure drop within the operating conditions used, if available.

2) With the measured overall gas holdup value (assumption 1: cross-sectional gas holdup $\left(\overline{\varepsilon_{G}}\right)=$ overall gas holdup) and A, calculate $\overline{\varepsilon_{\mathrm{S}}}$ from equation (8).

3) Calculate $\overline{v_{S}}$ using equation (9).

4) With the known value of $\varepsilon_{S}^{0}$, use equations 11 , and 10 , to calculate the gas and solids holdup cross-sectional distributions, $\varepsilon_{\mathrm{G}, \mathrm{ij}}$, and, $\varepsilon_{\mathrm{S}, \mathrm{ij}}$, respectively.

5) By azimuthally averaging the distributions obtained in step 4), calculate the gas and solids radial profiles, $\varepsilon_{\mathrm{G}, \mathrm{r}}, \varepsilon_{\mathrm{S}, \mathrm{r}}$, respectively.

6) Calculate the cross-sectionally averaged gas and solids holdups, $\overline{\varepsilon_{\mathrm{G}}}$ and $\overline{\varepsilon_{\mathrm{S}}}$ (radial averaging).

7) Check whether the value of the calculated cross-sectional gas holdup and the measured overall gas holdup are within the preset tolerance $\left(10^{-4}\right)$, Otherwise, change the value of A and repeat steps (2) to (6) until the convergence criterion is achieved (i.e., calculated cross-sectional gas holdup $\left(\overline{\varepsilon_{G}}\right)=$ overall gas holdup within the preset tolerance).

The predicted gas and solids holdup profiles have been evaluated by the overall solids mass balance and found to be satisfactory. 


\section{RESULTS AND DISCUSSION}

\section{Effect of Superficial Gas Velocity}

Figures $3 a$ and $b$ show the effect of superficial gas velocity on the gas and solids holdup profiles in airTherminol LT-glass beads system at atmospheric pressure. Due to the increase in overall gas holdup with superficial gas velocity, the magnitude of gas holdup profile also increases. With an increase in superficial gas velocity the system tends to get into churn-turbulent flow regime where gas holdup profile becomes parabolic. The effect of superficial gas velocity on the solids holdup profile is not much significant as compared to gas holdup, which would be due to the assumption of uniform cross-sectional solids loading.

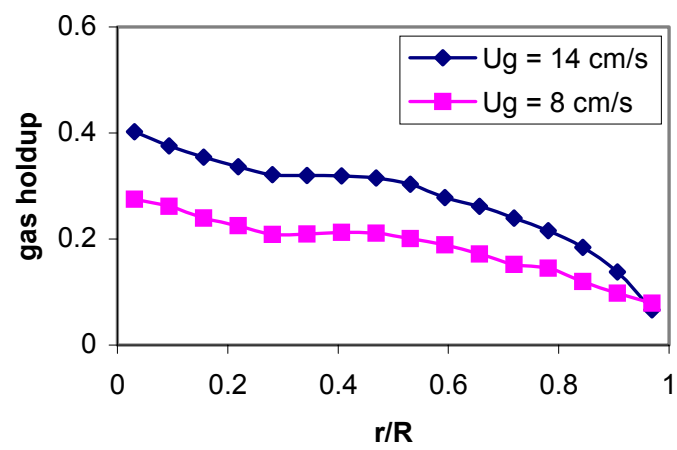

(a)

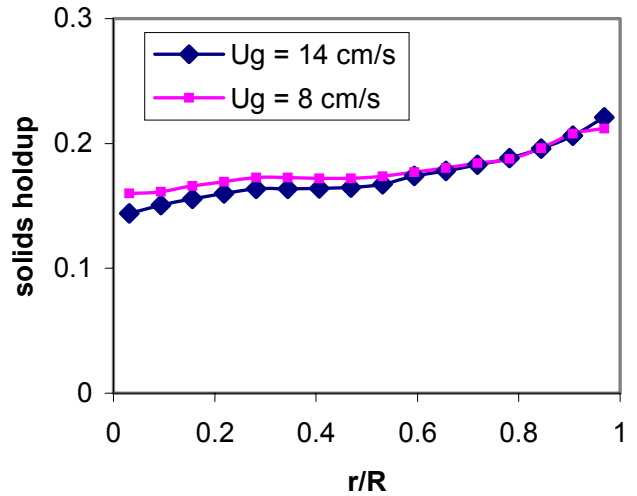

(b)

Figure 3: Effect of superficial gas velocity on a) gas holdup profile and b) solids holdup profile in air-Therminol LT-glass beads with $9.1 \%$ vol. solids loading at $0.1 \mathrm{MPa}$ in 6 inch column

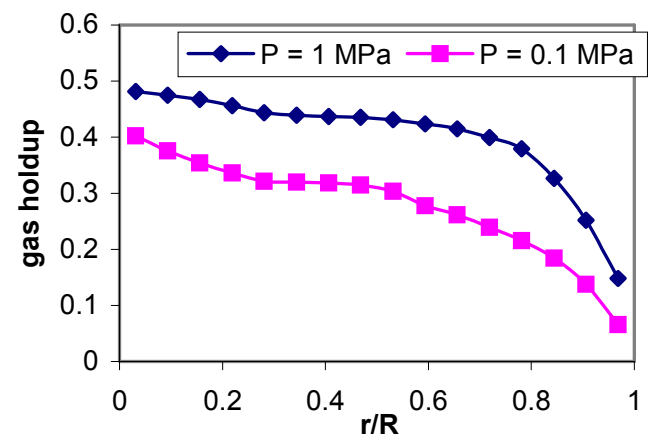

(a)

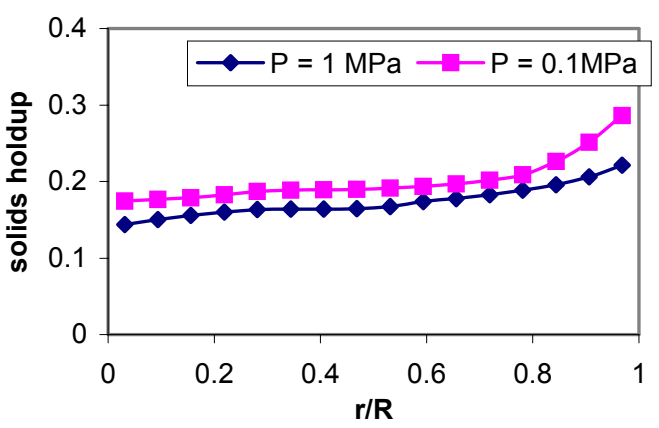

(b)

Figure 4: Effect of operating pressure on a) gas holdup profile and b) solids holdup profile in air-Therminol LT-glass beads with $9.1 \%$ vol. solids loading at superficial gas velocity of $14 \mathrm{~cm} / \mathrm{s}$ in 6 inch column

\section{Effect of Operating Pressure}

Figures $4 a$ and $b$ shows the effect of operating pressure on gas holdup and the solids holdup profile at a superficial gas velocity of $14 \mathrm{~cm} / \mathrm{s}$ in an air-Therminol LT-glass beads system. With an increase in pressure, the bubble break up rate increases, while the coalescence rate decreases which leads to smaller bubble sizes and subsequently into an increase in gas holdup (Wilkinson, 1993). This results in higher gas holdup profile with an increase in operating pressure at the same superficial gas velocity. The solids holdup profile decreases with an increase in pressure as a result of an increase in gas holdup. The 
effect of pressure on solids holdup profile is found to be less significant compared to the gas holdup profile.

\section{Effect of Physical properties of liquid}

The effect of physical properties of the liquid has been studied by using water and Therminol LT. Figure 5 shows gas holdup profiles in an air-water-glass beads system (Rados, 2003) and an air-Therminol LTglass beads system at atmospheric pressure and superficial gas velocity of $14 \mathrm{~cm} / \mathrm{s}$. The lower viscosity leads to a relatively unstable interface, resulting in a higher bubble breakup rate than coalescence rate. As well, lower surface tension results into a higher break up rate. Both of these factors give higher gas holdup in air-Therminol LT-glass beads than air-water-glass beads system and subsequently result into higher gas holdup profiles in air-Therminol LT-glass beads.

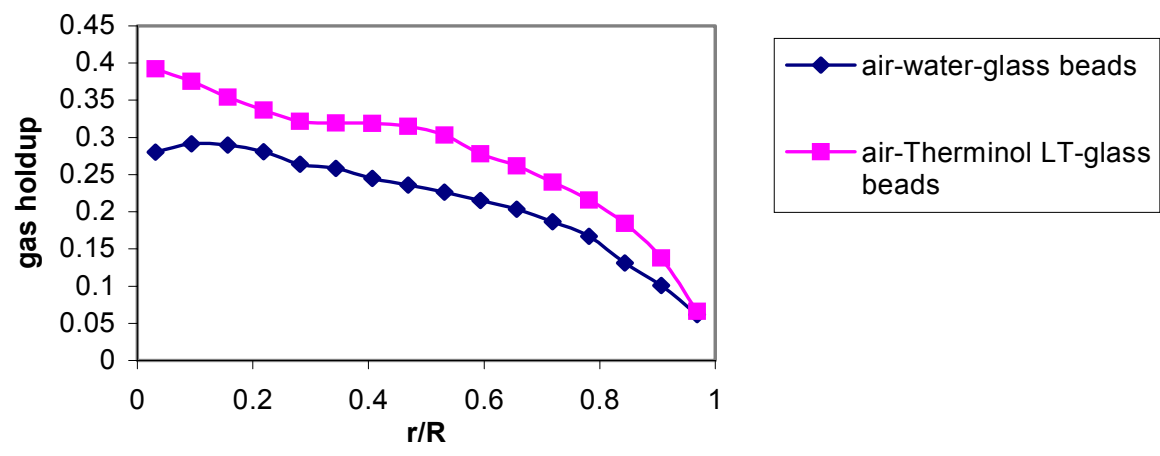

Figure 5: Radial profile of gas holdup in air-water-glass beads and air-Therminol LT-glass beads system with $9.1 \%$ vol. solids loading in 6 inch column at superficial gas velocity of $14 \mathrm{~cm} / \mathrm{s}$ at $0.1 \mathrm{MPa}$

\section{CONCLUSION}

A new methodology based on sound assumptions has been developed to calculate phase holdups in reactors with three dynamic phases. The method uses single $\gamma$-radiation source CT measurements combined with overall gas holdup measurement and sound assumptions. The effect of superficial gas velocity $(8-14 \mathrm{~cm} / \mathrm{s})$ and operating pressure $(0.1-1 \mathrm{MPa})$ on gas and solids holdup profiles have been studied using the developed methodology. It has been found that increase in superficial gas velocity and operating pressure cause a considerable increase in gas holdup profiles. The effect on the solids holdup profile appears to be insignificant within studied operating range. Also, a fluid with low viscosity and low surface tension was found to give a relatively higher gas holdup profile.

\section{ACKNOWLEDGEMENT}

The authors are thankful for the DOE - UCR grant (DE-FG-26-99FT40594) which made this work possible. 


\section{NOMENCLATURE}

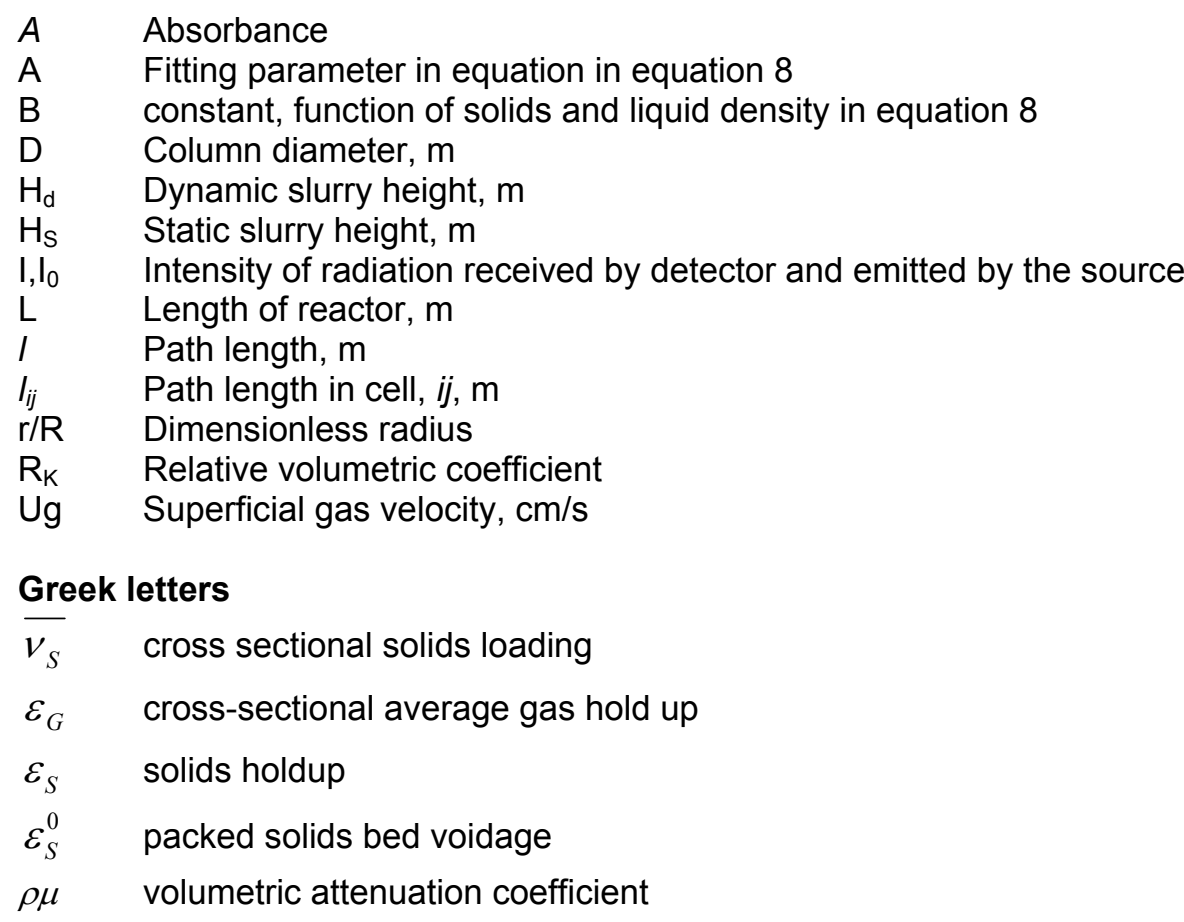

\section{REFERENCES}

CHAOUKI J., LARACHI F. and DUDUKOVIC M. P., (1997), Non-invasive monitoring of multiphase flows, Elsevier Science B. V., Amsterdam, Netherlands.

CHEN J., RADOS N., AL-DAHHAN M. H., DUDUKOVIC M. P., NGUYEN D. and PARIMI K., (2001), Particle motion in packed/ebullated beds by CT and CARPT, AIChE J., 47, 994.

Kemoun, A., Ong, B.C., Gupta, P., Al-Dahhan, M.H., \& Dudukovic, M. P., (2001), Gas holdup in bubble columns at elevated pressures via computed tomography. International Journal of Multiphase Flow, 27, 929.

KUMAR, S. B. and DUDUKOVIC, M. P., (1997), Computer assisted gamma and X-ray tomography: applications to multiphase flow systems. in Non-Invasive Monitoring of Multiphase Flows, 47, Elsevier Science B. V., Amsterdam, Netherlands.

RADOS N., (2003), Slurry bubble column hydrodynamics: experimentation and modeling. D.Sc. Thesis, Washington University, St. Louis, MO.

KRISHNA R., DE SWART J. W. A., ELLENBERGER J., MARTINA G. B., MARETTO C., (1997), 'Gas Holdup in Slurry Bubble Columns: Effect of column diameter and slurry concentrations,' AIChE J., 43, 2, 311-316.

WARSITO, OHKAWA M., MAEZAWA A. and UCHIDA S., (1997), Flow structure and phase distributions in a slurry bubble column. Chem. Eng. Sci., 52, 3941.

WARSITO W. and FAN L.-S., (2001a), Neural networks based multi-criterion optimization image reconstruction technique for imaging two- and three-phase flow systems using electrical capacitance tomography, Meas. Sci. Technol., 12, 2198.

WILKINSON P. M., VAN SCHAYK A., SPRONKEN J. P. M. and VAN DIERENDONCK L. L., (1993), The influence of gas density and liquid properties on bubble breakup. Chem. Eng. Sci., 48, 1213. 


\section{$\underline{\text { Appendix F }}$}

\section{Technical Difficulties and Improvements in CARPT}


During the process of implementing CARPT for the first time on high pressure steel column, several serious technical problems and issues have been arised, which need to be investigated and resolved carefully. Fortunately, all these problems have been successfully identified and resolved to implement CARPT technique at high pressure operation partly as a part of this project and other projects (Industrial Consortium and DF-22-95PC95051) which are executed in parallel with this grant. We will briefly outline the problems and their implemented solutions.

\section{Sc 46 CARPT Tracer Particle:}

As mentioned earlier the radioactive particle should, as closely as possible, track the solids present in the system. To accomplish this, the tracer particle should be of comparable size and density to the solid phase particles. Scandium is a highly reactive rare earth metal whose reactivity increases with increase in surface area per unit volume i.e. decrease in diameter. In addition Sc is highly reactive with water and oxygen. To resolve the issue of reacting scandium tracer particle, we have developed a new technique for coating and protecting the minute size tracking particles. The Sc 46 particle is protected with inert material, parylene which is a derivative of poly $\mathrm{p}$-xylene. The reasons for parylene as a choice for coating are,

- $\quad$ high melting point

- $\quad$ radiation resistance

- $\quad$ excellent mechanical properties -comparable to epoxy resins

- $\quad$ can be applied in layers below $0.1 \mu \mathrm{m}$

- $\quad$ small density

- $\quad$ solvent resistance

- $\quad$ low permeability to moisture and gases

- $\quad$ no outgassing

- $\quad$ acid and base resistance

- $\quad$ uniform thickness on all surfaces

There are four types of parylenes, among which Parylene $\mathrm{N}$ was selected based on the specifications listed in Table 1.

Table 1: Specifications of some Parylene family

\begin{tabular}{|c|c|c|c|c|}
\hline & $\operatorname{Tm}\left({ }^{\circ} \mathrm{C}\right)$ & $\begin{array}{c}\text { Stable in } \\
\text { oxygen }\left({ }^{\circ} \mathrm{C}\right)\end{array}$ & Density $(\mathrm{g} / \mathrm{cm} 3)$ & $\begin{array}{c}\text { Atoms other than C } \\
\& \mathrm{H}\end{array}$ \\
\hline Paralyne N & 410 & 115 & 1.11 & None \\
\hline Paralyne C & 290 & 125 & 1.29 & $1 \mathrm{Cl}$ \\
\hline Paralyne D & 380 & 150 & 1.42 & $2 \mathrm{Cl}$ \\
\hline Paralyne HT & 500 & 450 & 1.58 & $4 \mathrm{~F}$ \\
\hline
\end{tabular}

During coating, ionized air is blown over the particles to remove static electricity. On the walls of tumbler (core machine \#1 with $7.5 \times 4.5$ " tumbler), static guard is sprayed to reduce static charge further. Then tumbler with particles is closed by masking tape. The required quantity of 
Parylene $\mathrm{N}$ dimmer is put in the system and is slowly vacuumed to $50 \mu \mathrm{m}$ water and door \& vaporizer temperature are set to $125^{\circ} \mathrm{C}$. The rotational speed of tumbler was set to $4 \mathrm{rpm}$. This process has been performed by Paratech Inc.

Amongst the coated particle, several 'good' particles were selected for the irradiation on the basis of required size, shape and coating quality using microscope.

The irradiation of the selected particles was conducted at the flux of $4 \mathrm{e} 14 \mathrm{n} / \mathrm{cm} 2 . \mathrm{sec}$ at ORNL (Oak Ridge National Laboratory) Nuclear reactor.

Figure 1 shows typical pictures of the coated tracer particle.

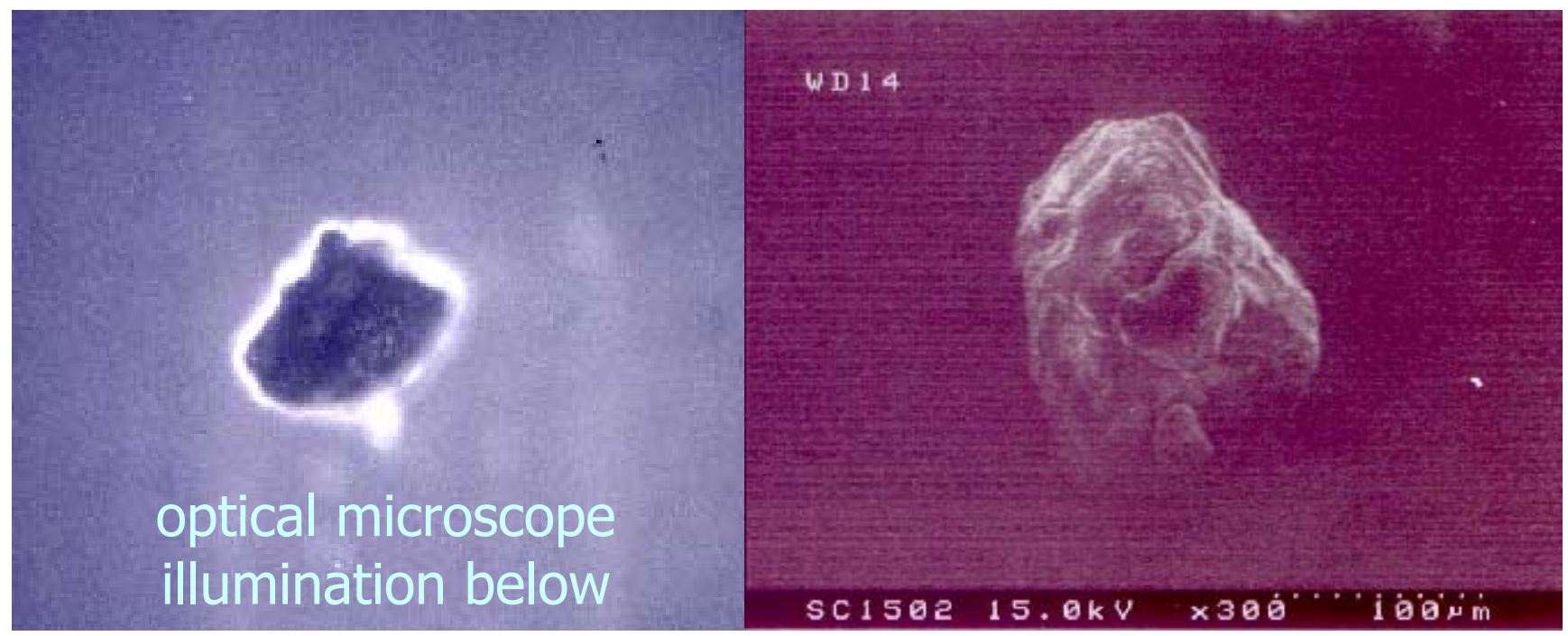

Figure 1: Typical pictures of tracer particles

\section{CARPT calibration in high pressure slurry stainless steel column}

A reliable calibration technique is the one that assures accurate positioning of the particle which can be read with enough precision. In all CARPT experiments automated system was used. But due to the presence of the solids as the third phase, high reactor pressure and the flange design of high pressure calibration devise, the calibration devise failed to operate properly. Mechanical modifications have been implemented successfully to resolve these problems. In addition, several technical issues in the process of CARPT implementation have been encountered that affect considerably the accuracy of reconstructing the position of the radioactive particle. These problems as well have been successfully identified and resolved as outlined below. 


\section{i) Build-up problems}

For a given tracer particle radioactivity level number of received counts by the detector is a function of distance, solid angle (spatial view angle of a detector by the tracer particle) and medium composition along the $\gamma$ ray path. The three most widely used radionuclides in Radioactive Particle Tracking: Sc46, Co60 and Au198, are $\beta$-decay electron emission proton deficient atoms that emit photoelectric $\gamma$ rays of the energy of about $1 \mathrm{MeV}$. The 0.1 to $1.0 \mathrm{MeV}$ energy range $\gamma$ radiation spectrum can be divided into two regions, as illustrated in Figure 2. Compton scattering is the first region with low energy photons that are subject to heavy scattering in highly attenuating mediums (high coefficient of $\gamma$ radiation attenuation). In these interactions the photon undergoes a billiard-ball type collision with electrons of the interacting medium. High energy photon portion of the spectrum contains one or two so-called photo-peaks whose energy corresponds to the photoelectric absorption energy. In these interactions the energy of the photon is completely absorbed by the electron of the interacting medium causing the kinetic energy of the electron to increase.

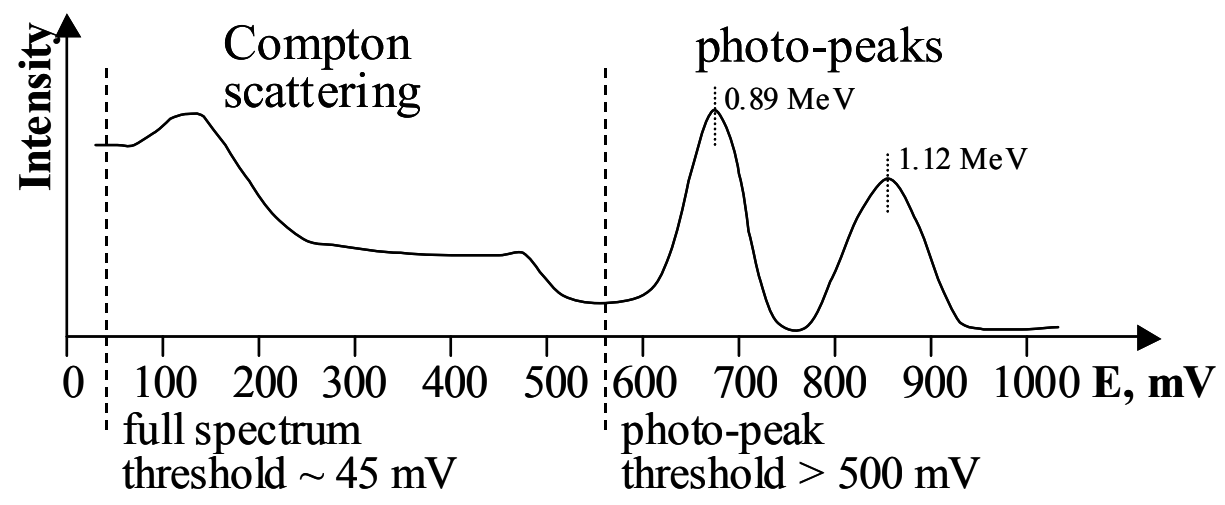

Figure 2: Scandium Sc46 $\gamma$ Energy Spectrum

Prior to this work, at CREL CARPT was exclusively done in Plexiglas columns and CARPT data acquisition used the photons of the whole spectrum (15 to $45 \mathrm{mV}$ threshold). In the present work highly attenuating (high density) stainless steel column wall and glass beads heavily scatter the Compton scattering photons. The scattering is so high that the system attenuation can not be any more deterministically modeled using the Lambert-Beer's law causing the wide and ill shaped CARPT calibration curves. The way to overcome this problem is to use only the high energy photo-peak photons. This is done by setting the threshold to be at the local intensity minimum at the energy just below the low energy photo peak (Figure 2, threshold $\sim 560 \mathrm{mV}$ ). This approach is novel at CREL, in G-L or G-L-S systems but had been already used by Larachi et al. (1994) in low loading G-S systems. This approach may only be used when the photo-peaks are at fixed positions, i.e., when the spectrum is well defined and stable. Potential disadvantage of this approach in low attenuating systems is that since only the fraction of the energy spectrum is acquired much less counts are received causing the tracer particle location reconstruction errors to be higher due to the lower signal-to-noise $(\mathrm{S} / \mathrm{N})$ ratio. 
The spectrum is stable when the data acquisition system is well grounded, at constant temperature and without excessive electrical resistances caused by poor electronic connections. In addition, detectors have to be functioning properly with good crystal and the detector power supply has to be stable for an extended time (e.g., overnight). Under these conditions spectrum will shift slightly only when significantly different count rates are received. Such as if the tracer particle of an order of magnitude different activity is used. Or if the tracer particle - detector distance is excessively changed, such as for more than $70 \mathrm{~cm}$ in the present air-water system. When the medium is changed from air to water although the intensity of detected radiation falls for 40 to $50 \%$ no appreciable energy shift of the photo-peaks may be observed. Once stabilized detector spectrum may drift slightly over time. The drift is typically smaller than $\pm 10 \mathrm{mV}$ per day and does not affect the measurements. Still, to be sure that the detectors are well adjusted before each CARPT experiment, fine tuning of the photo-peaks to the predetermined energy positions are performed.

\section{iii) Detector alignment}

Previously, detectors at CREL were aligned visually within "couple of millimeters". This poor alignment was tolerable in low attenuating Plexiglass bubble columns. However, in the present stainless steel column because of large attenuation of the wall detectors must be aligned much more accurately. This is done using a newly developed and much more accurate detector laseralignment. Detectors are horizontally leveled using a leveling device and are aligned in the axial and azimuthal direction using the laser equipped PVC dummy detector. The detector radial position is set using the cylindrical PVC spacer of the required thickness to adjust the gap between the detector face and the column. Using newly developed alignment procedure detectors are aligned within $1 \mathrm{~mm}$ axially and radially and within 0.5 degree azimuthally.

\section{iv) Pile-up problems}

Poor relative orientation of source and detector can cause detectors to be 'blinded' by excessive counts or receiving very low counts. When the tracer particle is very close to the detector, the gamma photons have a high probability of interaction with the detector crystal. Hence, many photons are counted while in the case when the particle radiation source moves away from the detector axis, a region of steep slope of counts versus distance is encountered which is characterized by large changes in counts with the small changes in distance. Also, there is asymptotic region in which large changes in distance make only small variations in measured counts. In this region, detectors are placed flush to the wall to avoid saturation of photons on crystal surface. As a result, placing the detector in such region means using it to the full scale.

\section{v) Modification in existing reconstruction algorithm in slurry Stainless Steel column}

Inappropriate reconstruction algorithm can also cause the error. The algorithm may not be utilizing the attenuation information obtained during the calibration completely or correctly. 
Reconstruction algorithm error is usually correlated and may be biased. The improvements in data processing using newly developed algorithm have been discussed below.

The simple way to test the accuracy of a reconstruction algorithm is to reconstruct the known calibration locations of the tracer particle using the time-averaged number of counts obtained from each of these calibration locations during the CARPT calibration. Acquisition of 192 scans (3.84 seconds) at the single calibration tracer location is long enough to average out the effects of: the statistical nature of the $\gamma$ radiation, electrical noise, holdup fluctuations and the dynamic bias (if present). The only two effects that can be evaluated using this error estimation procedure are the reconstruction algorithm error and the experimental error. In this procedure, first, the distance vs. (time-averaged) intensity counts calibration curves are generated for each of the detectors. Then, the time-averaged number of counts received by the detectors from the tracer particle at each of the calibration locations is used to reconstruct the tracer time-averaged "trajectory" during the calibration experiment. The error caused by the reconstruction algorithm can then be evaluated comparing the actual and the reconstructed tracer particle trajectories.

Comparison of the reconstructed and actual tracer particle locations in $r-\theta$ space by using the existing (Degaleesan, 1997) and the new algorithm is given in Figure 3 for the gas-liquid system and in Figure 4 for the gas-liquid-solid system. In these figures multiple reconstructed timeaveraged points at a given $(r, \theta)$ location correspond to different axial calibration levels. From Figure 3, it is clear that the new algorithm reconstructs the tracer particle calibration locations much more accurately. The reconstructed calibration locations using the new algorithm (dots) are well centered around the actual tracer particle locations (crosses) and are very little scattered. Degaleesan's algorithm results in much larger scattering of the reconstructed calibration locations. Especially reconstructed locations that are close to the wall are not well centered around the actual locations.

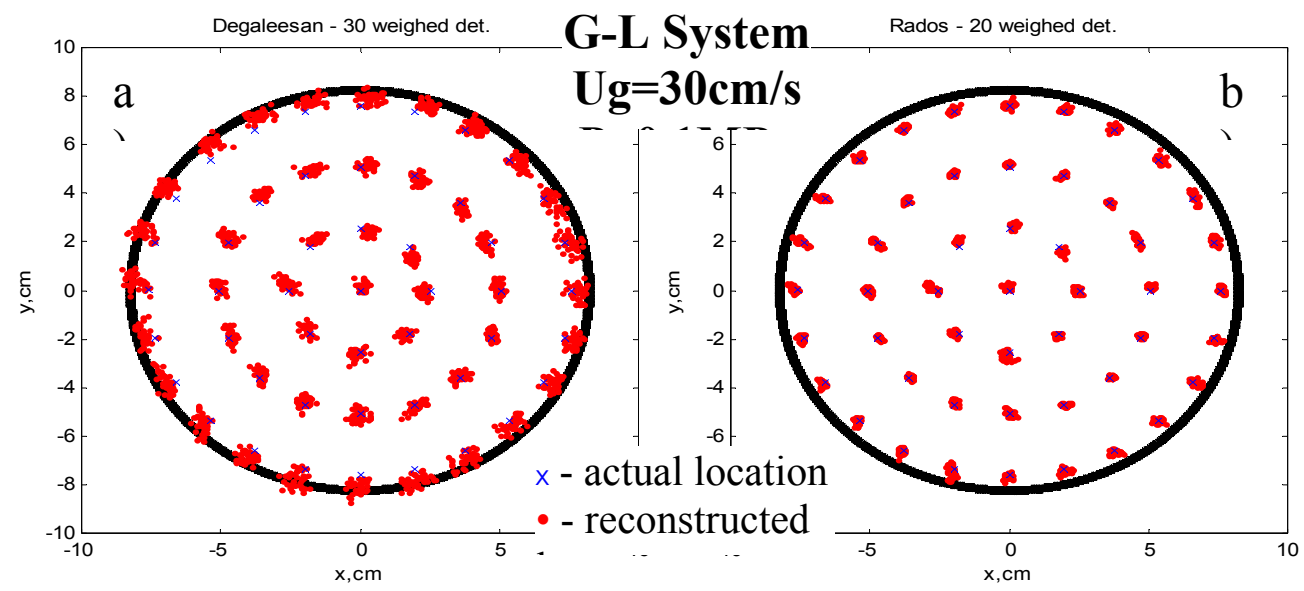

Figure 3: Comparison of the Reconstruction Errors for the Calibration Experiment in the G-L System at SGV of $30 \mathrm{~cm} / \mathrm{s}$ and Atmospheric Pressure. a) Degaleesan (1997), b) Present Study (Rados, 2003) 


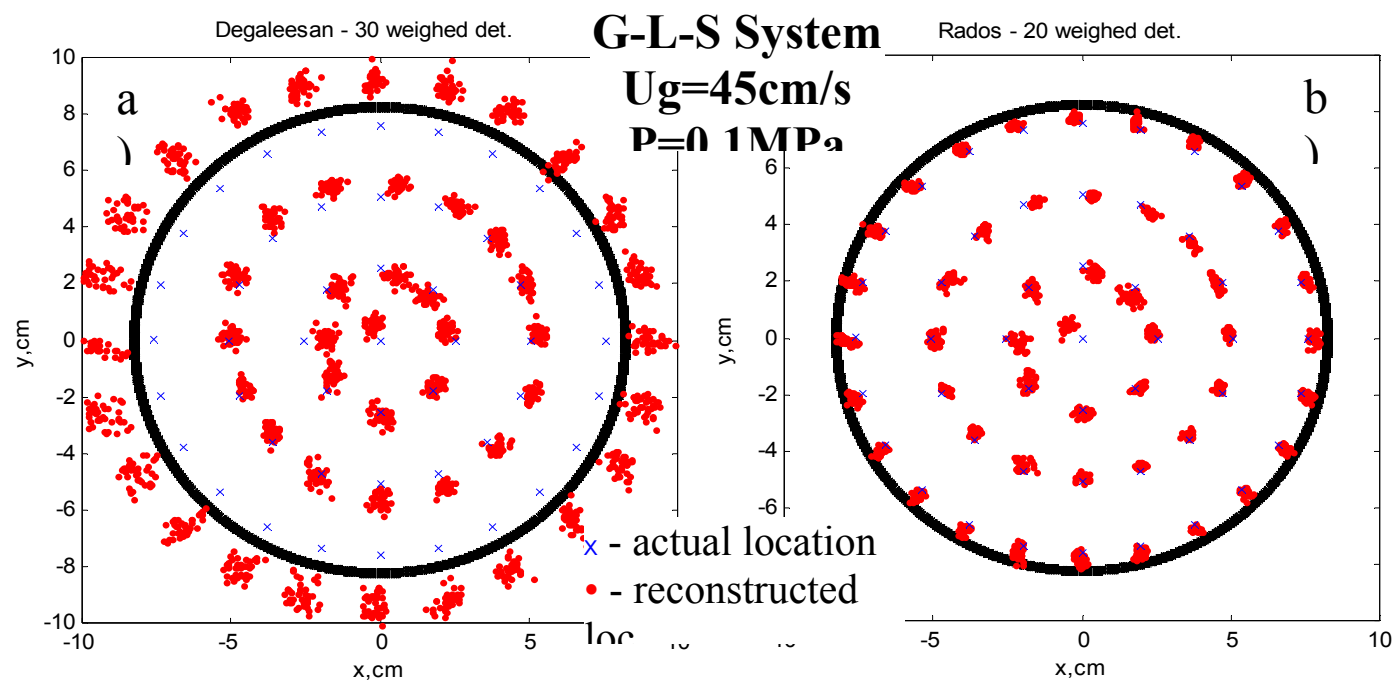

Figure 4: Comparison of the Reconstruction Errors for the Calibration Experiment in the G-L-S System at SGV of $45 \mathrm{~cm} / \mathrm{s}$ and Atmospheric Pressure. a) Degaleesan (1997), b) Present Study (Rados, 2003)

This is a clear indication that the Degaleesan's algorithm does not account well for the stainless steel wall path attenuation effects (wall shielding). Comparison of the reconstructed locations for slurry system in Figure 4 further proofs superiority of the new over Degaleesan's algorithm. The reconstruction of the wall region calibration points in the slurry system involves intolerable errors.

The error in any direction can be defined as $e_{p}=p-p_{\text {act }}$, where $p=r, \theta$, z. For the slurry system reconstruction errors are specially large in the wall region because of the wall path attenuation and also because of the solids attenuation whose holdup is expected to be the largest close to the wall. In the slurry systems wall shielding is always connected with the solids shielding. Both of these effects cause the lateral arcs to be so prominent on the calibration curves in slurry systems as shown in figure 5. Obviously the new reconstruction algorithm accounts for the presence of arcs much more effectively than the previous algorithm. 

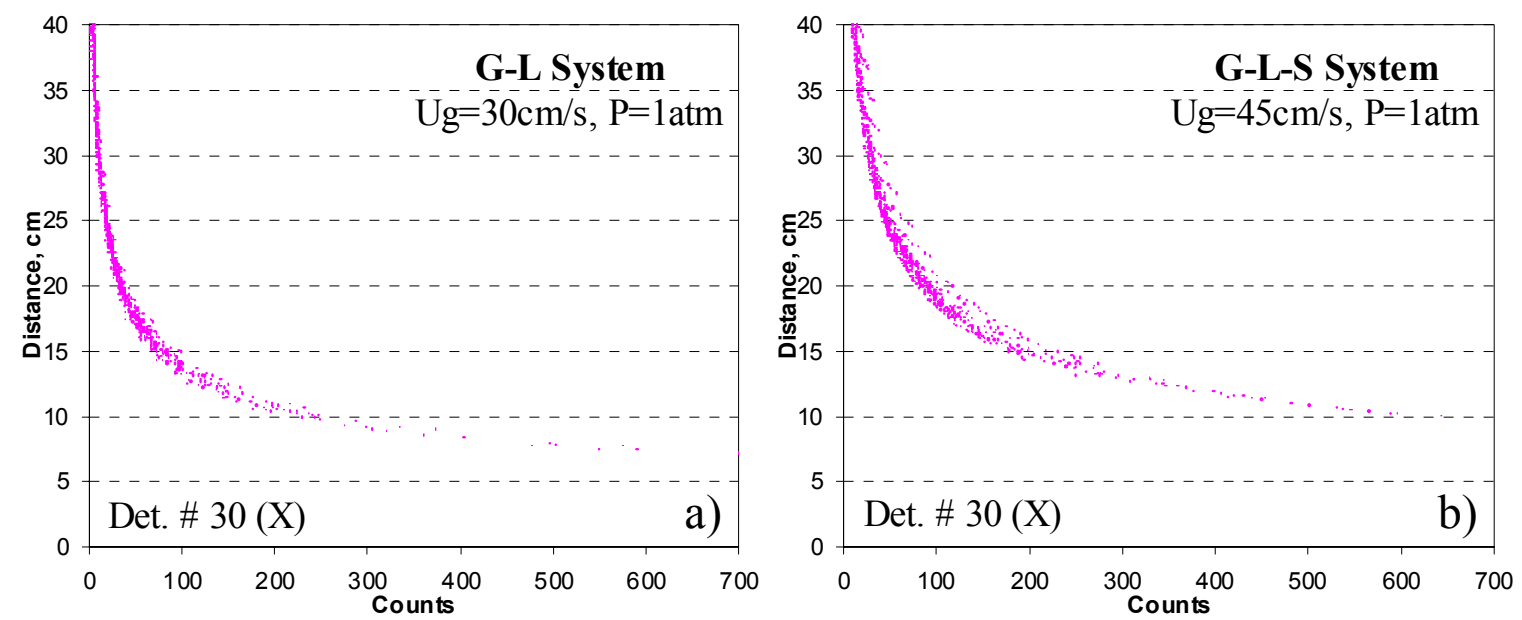

Figure 5: Distance vs. Counts CARPT Calibration Curves in a Stainless Steel Bubble Column. (a) G-L System, (b) G-L-S Slurry System

The central point in the Figure 4 is probably off because of the experimental not the reconstruction algorithm error. Actual calibration locations (crosses) are the locations where the tracer particle was intended to be placed, however it is possible that small error $(\sim 6 \mathrm{~mm})$ was made during the calibration. In all other performed experiments central point was always reconstructed with about the same accuracy as all other calibration points. Other way to graphically illustrate the accuracy of the new reconstruction algorithm is by plotting the histograms of the calibration location reconstruction errors in the axial and radial directions. The reconstruction error histograms for the slurry experiment at $\mathrm{SGV}$ of $45 \mathrm{~cm} / \mathrm{s}$ and atmospheric pressure are given in Figure 6. The calibration location reconstruction errors ( $\mathrm{e}_{\mathrm{P}}$ ) are quantitatively summarized in terms of the mean and the standard deviation in axial $(\mathrm{p}=\mathrm{z})$ and radial $(\mathrm{p}=\mathrm{r})$ directions (Table 2$)$. The mean and the standard deviation of the reconstruction errors for $\mathrm{N}$ calibration locations are calculated as:

$\mu_{\mathrm{p}}=\frac{1}{\mathrm{~N}} \sum_{\mathrm{n}=1}^{\mathrm{N}} \mathrm{e}_{\mathrm{p}, \mathrm{n}}$

$\sigma_{\mathrm{p}}=\sqrt{\frac{\mathrm{N} \sum_{\mathrm{n}=1}^{\mathrm{N}} \mathrm{e}_{\mathrm{p}, \mathrm{n}}^{2}-\left(\sum_{\mathrm{n}=1}^{\mathrm{N}} \mathrm{e}_{\mathrm{p}, \mathrm{n}}\right)^{2}}{\mathrm{~N}(\mathrm{~N}-1)}}$ 

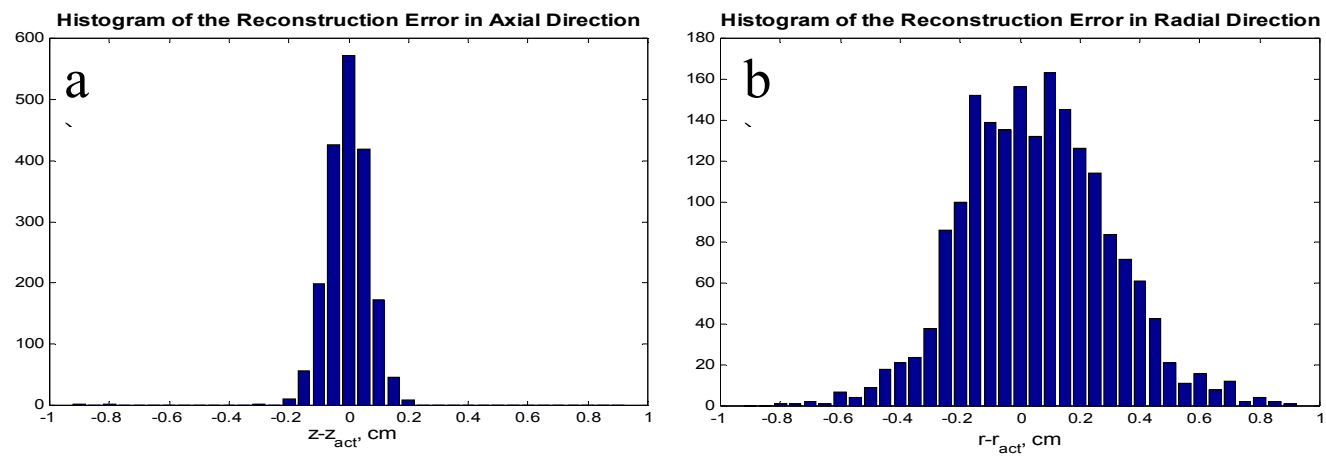

Figure 6: Histograms of the Reconstruction Errors in Axial (a) and Radial (b) Directions Obtained Using the New Reconstruction Algorithm. Calibration Experiment in the G-L-S System at $\mathrm{SGV}$ of $45 \mathrm{~cm} / \mathrm{s}$ and Atmospheric Pressure

Table 2: Reconstruction Errors for the Calibration Experiments in the G-L $\left(\mathrm{U}_{\mathrm{G}}=30 \mathrm{~cm} / \mathrm{s}, \mathrm{P}=0.1 \mathrm{MPa}\right)$ and G-L-S Systems $\left(\mathrm{U}_{\mathrm{G}}=45 \mathrm{~cm} / \mathrm{s}, \mathrm{P}=0.1 \mathrm{MPa}\right)$

\begin{tabular}{|c||c|c||c|c|}
\cline { 2 - 3 } \multicolumn{1}{c||}{} & \multicolumn{2}{c||}{ G-L-S, Ug $\mathbf{4 5} \mathbf{~ c m} / \mathbf{s}$} & \multicolumn{2}{c|}{ G-L, Ug $=\mathbf{3 0} \mathbf{~ c m} / \mathbf{s}$} \\
\hline $\begin{array}{c}\text { Averaged } \\
\text { Error }\end{array}$ & $\begin{array}{c}\text { Rados, } \\
\text { this work }\end{array}$ & $\begin{array}{c}\text { Degaleesan, } \\
1997\end{array}$ & $\begin{array}{c}\text { Rados, } \\
\text { this work }\end{array}$ & $\begin{array}{c}\text { Degaleesan, } \\
1997\end{array}$ \\
\hline$\mu_{\mathrm{Z}}, \mathbf{c m}$ & -0.0051 & -0.2815 & -0.0010 & 0.0223 \\
\hline$\sigma_{\mathbf{z}}, \mathbf{c m}$ & $\mathbf{0 . 0 7 7 7}$ & $\mathbf{0 . 4 4 2 1}$ & $\mathbf{0 . 0 7 2 7}$ & $\mathbf{0 . 2 5 1 8}$ \\
\hline$\mu_{\mathrm{r}}, \mathbf{c m}$ & 0.0549 & 0.8842 & 0.0596 & 0.1651 \\
\hline$\sigma_{\mathbf{r}}, \mathbf{c m}$ & $\mathbf{0 . 2 4 2 0}$ & $\mathbf{0 . 8 7 9 2}$ & $\mathbf{0 . 1 1 9 3}$ & $\mathbf{0 . 2 7 8 3}$ \\
\hline
\end{tabular}

From histograms in Figure 6 it is obvious that the reconstruction error in the radial direction is larger than in the axial direction. The same trend is also observed for the G-L experiment at SGV of $30 \mathrm{~cm} / \mathrm{s}$ and atmospheric pressure. Better accuracy in axial direction is observed regardless of the employed reconstruction algorithm (Table 2). Due to the large aspect ratio of the CARPT experiment control volume most of the detectors are closer to the tracer particle radially than axially. A consequence of this is that a small displacement in the axial direction has a larger effect on the tracer particle - detector distance than the same displacement in the radial direction (both absolutely and relatively with respect to the original distance). In other words a displacement in axial direction is more "obvious" to the detectors then the same displacement in the radial direction. Geometrically, a displacement of the tracer particle in given direction is the most "obvious" to the detector that is directly in line with the displacement. Hence, improvement of the radial accuracy with respect to axial accuracy would require addition of more detectors that are radially farther from the column than the existing detectors. This is counterintuitive as one would expect that packing more detectors as close to the column as possible would improve the accuracy the most. True, but this way both the radial and axial accuracy are increased, so that 
axial accuracy would still be better than the radial accuracy. Because of the geometrical reasons a comparable axial and radial accuracy can be achieved only in systems with aspect ratio of close to one and with detectors uniformly packed around the whole system. Same qualitative and quantitative trends observed in the slurry system at $\mathrm{SGV}$ of $45 \mathrm{~cm} / \mathrm{s}$ hold at lower values of SGV. Here for illustration purposes a slurry experiment at $S G V$ of $45 \mathrm{~cm} / \mathrm{s}$ is selected for illustration and is performed first only because it is the experiment that was the hardest to properly acquire and accurately reconstruct. At large SGV (like $45 \mathrm{~cm} / \mathrm{s}$ ) most of the solids particles are expected to be pushed towards the wall causing the largest solids/wall combined shielding effect (Badgujar et al., 1986; Hu et al., 1986; Limtrakul, 1996).

The performance of the newly developed reconstruction algorithm is also evaluated by reconstructing selected intermediate, non-calibration locations. During this evaluation experiment the tracer particle was placed at additional locations that are not used for generation of the calibration curves. These additional points were acquired upon the completion of the regular calibration experiment in slurry system with PPL sparger at SGV of $45 \mathrm{~cm} / \mathrm{s}$ and atmospheric pressure. For acquisition of these additional, non-calibration locations, the tracer particle was placed on the two axial levels: $109.27 \mathrm{~cm}$ (between the two detector levels with sufficient number of the surrounding detectors) and $149.91 \mathrm{~cm}$ (at the highest axial detector level). The extra points were placed on the two rings $\left(0.5\right.$ inch with the total of 8 points $45^{\circ}$ spaced and 2.5 inch with the total of 12 points $30^{\circ}$ spaced) as shown in Figure 7 . The reconstruction errors are slightly larger than for the calibration points, specially in the azimuthal direction. All of the reconstructed additional points at ring 2.5 appear to lay about $-6^{\circ}$ off. This could be due to the reconstruction algorithm error. However, it is believed that the larger disagreements observed at the intermediate and the center line calibration points was caused by miss-positioning of the calibration device that was not accurate due to the clogged with solids tripod springs.

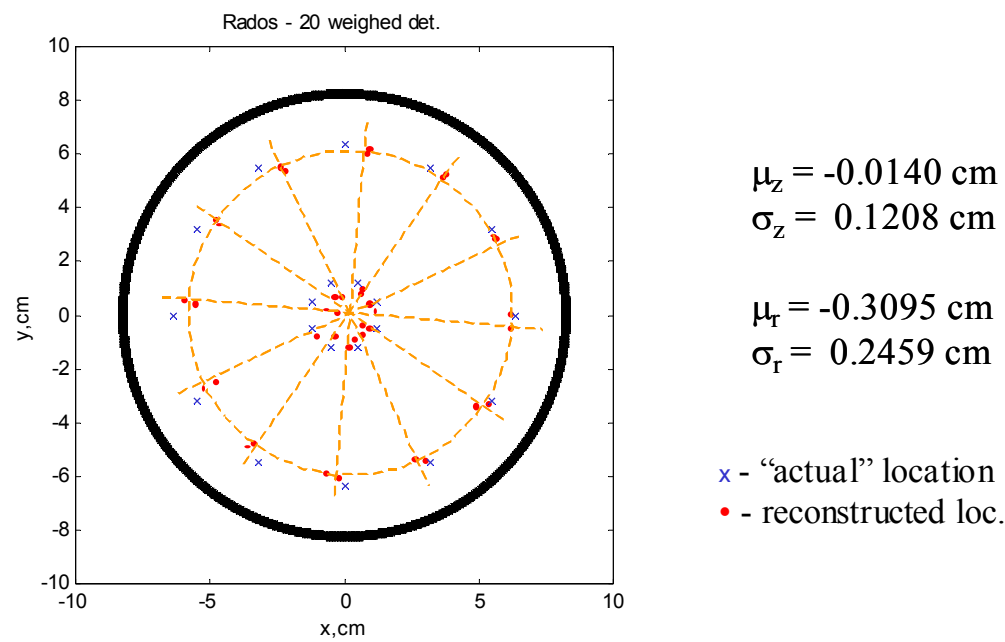

Figure 7: Comparison of the Reconstruction Errors for the Check Non-Calibration Points in the G-L-S System at SGV of $45 \mathrm{~cm} / \mathrm{s}$ and Atmospheric Pressure Using the New Reconstruction Algorithm (Rados, 2003). 
As can be seen all of these points still lay on circle but which is slightly smaller than 2.5 inch in diameter and slightly off the column axis. Typical result of less than $1 \mathrm{~cm}$ diagonally missaligned calibration device. In all of the later conducted experiments, after the tripod modification and further calibration device design advancements, the center line calibration points were properly centered with the errors (mean and standard deviation) that are comparable to the errors observed for the calibration locations.

To summarize, the reconstruction errors are always larger in radial and azimuthal directions than in the axial direction. The mean error in the radial direction is always quite small and positive $(+0.5$ to $+1 \mathrm{~mm})$. However, the standard deviation in the radial direction are of the order of couple of millimeters. In some experiments the histogram of the reconstruction errors in radial direction were slightly skewed to lower error values with the tail in positive direction. Calibration locations reconstructed using the new algorithm are much more accurate than when using the algorithm of Degaleesan (1997). Using the present algorithm the spheres of uncertainty in the tracer particle location reconstruction are about 2.5 times smaller in G-L and about 4 times smaller in slurry experiments than those obtained using the Degaleesan's algorithm.

\section{REFERENCES}

Degaleesan, S., Turbulence and liquid mixing in bubble columns, D.Sc. Thesis, Washington University, St. Louis, MO, 1997.

Larachi, F., Kennedy, G. and Chaouki, J. (1994). A $\gamma$-ray detection system for 3-D particle tracking in multiphase reactors. Nucl. Instr. \& Meth., A338, 568.

Badgujar, M. N., Deimling, A., Morsi, B. I., Shah, Y. T. and Carr, N. L. (1986). Solids distribution in a batch bubble column. Chem. Eng. Commun., 48, 127.

$\mathrm{Hu}, \mathrm{T} ., \mathrm{Yu}, \mathrm{B}$. and Wang, Y. (1986). Holdup and models of three phase fluidized bed. Fluidization $V, 353$.

Limtrakul, S. (1996). Hydrodynamics of liquid fluidized beds and gas-liquid fluidized beds. D.Sc. Thesis, Washington University, St. Louis, MO.

Rados, N., Slurry bubble column hydrodynamics, D.Sc. Thesis, Washington University, St. Louis, 2003. 